\title{
Image Separation using Multi-layer Image Segmentation for Translucent Partially Overlapped Objects
}

by

Tayebeh Lotfi Mahyari, M.Sc.

submitted to the

Faculty of Graduate and Postdoctoral Affairs

in partial fulfillment of the requirements for the degree of

PhD Thesis in Systems and Computer Engineering

Ottawa-Carleton Institute for Electrical and Computer Engineering

Department of Systems and Computer Engineering

Carleton University

Ottawa, Ontario

April 2021

CCopyright

Tayebeh Lotfi Mahyari, 2021 


\section{Abstract}

One way to solve under-determined image separation is to use statistical information about the type of data to be decomposed. In this dissertation, we propose a two stage method for cervical cell separation. In the first stage, we propose a $\mathrm{CNN}$-based multi-layer random walker image segmentation method. The results of image segmentation at the first stage are then used as the side information for the cervical cell separation in the second stage.

Convolutional neural networks (CNNs) are recently used in computer vision applications such as image segmentation. One of the biggest advantages of CNNs is that they extract important features automatically from the data. However, CNNs usually use a high number of data for training but it is not always possible to find enough training data for some applications. Also, CNNs are good at generalizing the training, but not for finding the accurate edges of cervical cells at the current implementations for cervical cell segmentation.

One solution to improve CNN segmentation results is to use a post-processing method as edge refinement. Random walker image segmentation method is a graph-based image segmentation method that is good at region growing; so, it can be used as the refinement step. However, random walker is sensitive to initial setup, so if the seeds are not extracted correctly, the final segmentation

results will be poor. In this dissertation we use a CNN-based random walker image segmentation approach for cervical cell segmentation. Different from other methods that use CNN binary segmentation results for fine tuning, CNN probabilistic map is utilized to guide random walker image segmentation method at the refinement step. 
In the second stage, we design a new $\mathrm{CNN}$, and we use the input image along with the segmentation masks from our first stage for cervical cell separation. To the best of our knowledge, there is no work done for overlapping cervical cell separation. We apply our two-stage cervical cell separation algorithm on synthetic cervical cytology images which can open an opportunity to early cervical cancer detection. Results show segmentation accuracy of greater than $99.5 \%$ and separation SSIM score of greater than 0.93 . 
To my lovely husband, who patiently supported me during my program. 


\section{Table of Contents}

$\begin{array}{ll}\text { Abstract } & \text { ii }\end{array}$

Table of Contents $\quad$ v

List of Tables $\quad$ ix

List of Figures $\quad$ xi

$\begin{array}{ll}\text { Abbreviation } & \mathbf{x x}\end{array}$

1 Introduction $\quad 1$

1.1 Image Decomposition . . . . . . . . . . . . . . . . 1

1.1.1 Image Segmentation . . . . . . . . . . . . . . 3

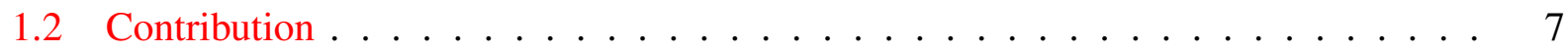

1.3 Dissertation Preview . . . . . . . . . . . . . . . 10

2 Background $\quad 11$

2.1 Partially Overlapped Translucent Objects . . . . . . . . . . . . . . . . 11

2.1 .1 X-ray Images . . . . . . . . . . . . . . . . . 11

2.1 .2 Cytology Images . . . . . . . . . . . . . . . . . . . 13

2.2 Image Segmentation . . . . . . . . . . . . . . . . . . . 14 
2.2.1 Graph Based Image Segmentation Methods . . . . . . . . . . . . . . 15

2.2.1.1 Markov Random Field . . . . . . . . . . . . . . . . 15

2.2.1.2 Probabilistic Image Segmentation . . . . . . . . . . . . 16

2.2.1.3 Random Walker Image Segmentation Algorithm . . . . . . . . . 17

2.3 Machine Learning, Deep Learning and Convolutional Neural Networks . . . . . 20

2.3.1 Deep Learning . . . . . . . . . . . . . . . . . . . . 20

2.3.1.1 Convolutional Neural Networks . . . . . . . . . . . . 21

2.3.1.2 Optimization on $\mathrm{CNNs} \ldots \ldots . \ldots 22$

2.4 CNN-based Image Segmentation . . . . . . . . . . . . . . . . . 23

2.4.1 AlexNet, ZFNet, VGGNet, GoogleNet, and ResNet . . . . . . . . . . . 23

2.5 Image Decomposition . . . . . . . . . . . . . . . . . 32

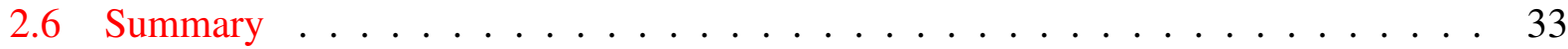

3 Multi-Layer Random Walker Image Segmentation 35

3.1 User-interactive Multi-Layer Image Segmentation . . . . . . . . . . . . . . 35

3.2 Learning-based Multi-Layer Random Walker Image Segmentation . . . . . . . . 40

3.2.1 Label Prior Calculation Method . . . . . . . . . . . . . . 41

3.2.2 Data Set-up for Multi-Layer Random Walker Image Segmentation . . . . . 45

3.2.2.1 Multi-layer User Input Random Walker Image Segmentation Results ............................. 46

3.2.2.2 Multi-layer Multi-label Random Walker Image Segmentation Using Prior Results . . . . . . . . . . . . . . . . . . 50



4 Multi-layer Image Segmentation using CNN 56

$4.1 \quad$ Proposed $\mathrm{CNN} \ldots \ldots \ldots \ldots \ldots \ldots$ 
4.1.1 Proposed CNN for Multi-layer Image Segmentation . . . . . . . . . . 58

4.2 Data Set-up for $\mathrm{CNN} \ldots \ldots \ldots$. . . . . . . . . . . . . . . 60

4.2.1 Data Set-up for Overlapped Image Segmentation . . . . . . . . . . . 61



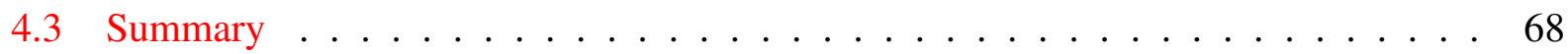

5 Multi-layer Random Walker Image Segmentation for Overlapped Cervical Cells using Probabilistic Deep Learning Methods $\quad 71$

5.1 Proposed CNN-based Image Segmentation for Overlapped Cervical Cells . . . . 72

5.1.1 Proposed Residual CNN . . . . . . . . . . . . . . . . 74

5.2 Jointly Overlapped Cervical Cell Segmentation _ . . . . . . . . . . . . . 77

5.2 .1 Data Set-up . . . . . . . . . . . . . . 77

5.2.2 Nucleus and Cytoplasm Mapping . . . . . . . . . . . . . . 79

5.2.2.1 Nuclei and Approximate Cytoplasm Detection . . . . . . . . . 81

5.2.3 Cells' Borders Fine Tuning . . . . . . . . . . . . . . 86

5.2.4 Performance Evaluation . . . . . . . . . . . . . . . . . 90

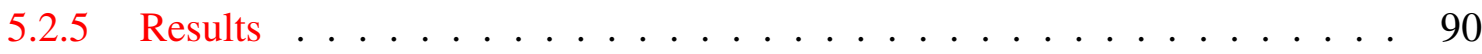

5.3 Multiple Overlapped Cervical Cell Segmentation . . . . . . . . . . . . . 95

5.3 .1 Data Set-up . . . . . . . . . . . . . . . . 95

5.3.2 Nucleus and Cytoplasm Mapping . . . . . . . . . . . . . . . 99

5.3.2.1 Nuclei and Approximate Cytoplasm Detection . . . . . . . . . . 99

5.3.3 Cells' Borders Fine Tuning . . . . . . . . . . . . . . 101

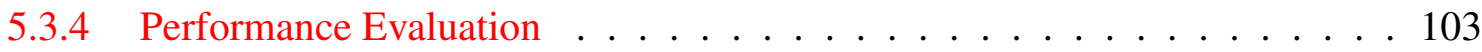

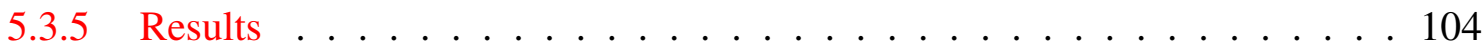

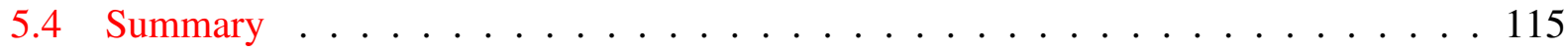


6 Proposed Two-stage CNN for Image Separation

6.1 Proposed $\mathrm{CNN} \ldots \ldots \ldots \ldots \ldots \ldots \ldots$

6.1 Two-Stage CNN for Image Separation . . . . . . . . . . . . . . 118

6.2 Data Setup . . . . . . . . . . . . . . . . . . 120

6.2.1 Two Overlapping Cells . . . . . . . . . . . . . . . . 120

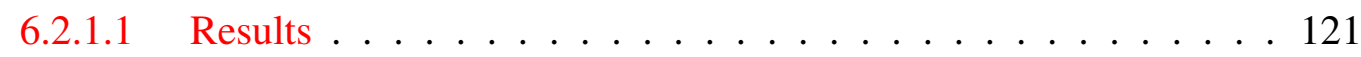

6.2.2 Jointly Overlapped Multiple Cervical Cells . . . . . . . . . . . . . . 127

6.2.2.1 Results ........................ 128

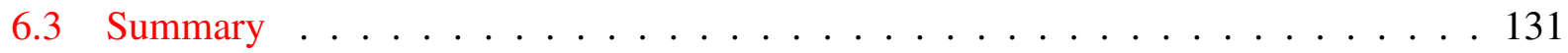

7 Conclusion and Future Work $\quad 133$

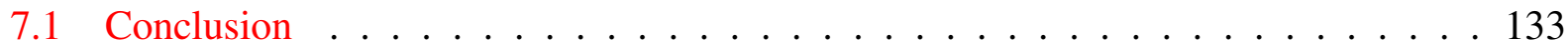

7.2 Future Work . . . . . . . . . . . . . . . . . 136

$\begin{array}{lr}\text { List of References } & 138\end{array}$

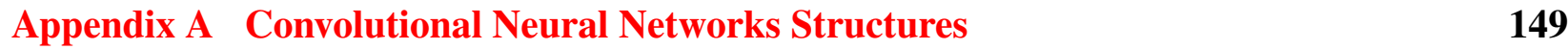

A.1 SegNet Network . . . . . . . . . . . . . . . . . . . . 149

A.2 Residual Network for Overlapped Image Segmentation . . . . . . . . . . . . 149

A.3 Residual Network for Image Decomposition . . . . . . . . . . . . . . . . . . . 149

$\begin{array}{lll}\text { Appendix B Supervised MRF image segmentation formulation } & 171\end{array}$

$\begin{array}{lll}\text { Appendix C Similarity Measures } & 173\end{array}$

$\begin{array}{lll}\text { Appendix D } & \text { Optimization on CNNs } & 176\end{array}$ 


\section{List of Tables}

3.1 Segmentation accuracy and time complexity for the multi-layer user input random walker image segmentation method and the original random walker image segmen-



3.2 Segmentation accuracy for our proposed multi-layer user input random walker image segmentation method comparing to the original random walker image segmentation $[1]$ on synthetic cervical images. . . . . . . . . . . . . . . . 51

3.3 Segmentation accuracy and execution time for our proposed multi-layer multi-label RW image segmentation method comparing with the original random walker image segmentation method [1], [2] and our proposed multi-layer user input RW image segmentation method [3]. . . . . . . . . . . . . . . . . 54

3.4 Segmentation accuracy and IOU of our proposed multi-layer multi-label RW image segmentation algorithm compared to our multi-layer user input RW image segmentation for synthetic cervical images. . . . . . . . . . . . . . 55

4.1 Segmentation accuracy. . . . . . . . . . . . . . . 65

4.2 Segmentation accuracy. . . . . . . . . . . . . . . 70

5.1 Segmentation quantitative measure. . . . . . . . . . . . . . . . 94

5.2 Training and test time complexity. . . . . . . . . . . . . . 96

5.3 Aggregate mean and standard deviation quantitative segmentation results for the proposed method and those in [4-7] based on a set of 100 test images. . . . . . . . 109 
5.4 Training times and test times. . . . . . . . . . . . . . . . . . . . 114

6.1 Segmentation quantitative measure. . . . . . . . . . . . . . . . . . . . . 124

6.2 Separation quantitative measure. . . . . . . . . . . . . . . . . . . 127

6.3 Separation accuracy. . . . . . . . . . . . . . . . . 131

A.1 SegNet, layers 1-12. . . . . . . . . . . . . . . . . . . . 150

A.2 SegNet, layers 13-24 . . . . . . . . . . . . . . . . . . . . . . 151

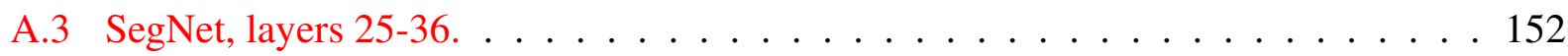

A.4 SegNet, layers $37-48 \ldots \ldots \ldots$. . . . . . . . . . . . . . . 153

A.5 SegNet, layers $49-60 \ldots \ldots \ldots$. . . . . . . . . . . . . . . . 154

A.6 SegNet, layers $61-72 \ldots \ldots \ldots 155$

A.7 SegNet, layers $73-84 \ldots \ldots \ldots \ldots$. . . . . . . . . . . . . . 156

A.8 SegNet, layers $85-91 \ldots \ldots \ldots \ldots$

A.9 Proposed residual network for image segmentation with 81 layers, layers 1-12 . . . 158

A.10 Proposed residual network for image segmentation, layers 13-24 . . . . . . . . . 159

A.11 Proposed residual network for image segmentation, layers 25-36 . . . . . . . . . 160

A.12 Proposed residual network for image segmentation, layers 37-47 . . . . . . . . . 161

A.13 Proposed residual network for image segmentation, layers 48-58 . . . . . . . . . 162

A.14 Proposed residual network for image segmentation, layers 59-70 . . . . . . . . . 163

A.15 Proposed residual network for image segmentation, layers 71-81 . . . . . . . . . . 164

A.16 Proposed residual network for image decomposition with 66 layers, layers 1-12. . . 165

A.17 Proposed residual network for image decomposition, layers 13-24 . . . . . . . . . 166

A.18 Proposed residual network for image decomposition, layers 25-36 . . . . . . . . 167

A.19 Proposed residual network for image decomposition, layers 37-48 . . . . . . . . 168

A.20 Proposed residual network for image decomposition, layers 49-60 . . . . . . . . 169

A.21 Proposed residual network for image decomposition, layers 61-66. . . . . . . . . 170 


\section{List of Figures}

1.1 X-ray image samples from airport security devices [8]. . . . . . . . . . . 5

1.2 Papanicolaou (PAP) red stain kit (cytology stain) [9]. . . . . . . . . . . . . . 6

2.1 An xray image sample from the airport security device [10]. . . . . . . . . . 13

2.2 Normal pap. Superficial cells with smaller nuclei, and intermediate cells (center) with larger nuclei are seen $[11] . \ldots \ldots \ldots \ldots \ldots$

2.3 Inception layer for GoogLeNet. . . . . . . . . . . . . . . . . . . . . . . . 24

2.4 Learning residual function for ResNet. . . . . . . . . . . . . . . . . . . 25

2.5 AlexNet structure. . . . . . . . . . . . . . . . . 26

2.6 ZFNet structure. . . . . . . . . . . . . . . . . 27

2.7 VGGNet structure. . . . . . . . . . . . . . . . . . . . . . 29

2.8 GoogLeNet structure. . . . . . . . . . . . . . . . . 30

2.9 ResNet structure. . . . . . . . . . . . . . . . . . . 30

2.10 SegNet network [12] for semantic image segmentation. . . . . . . . . . . 31 
3.1 $3 D$ lattice structure for a $2 D$ image $J$ with $N$ regions. An $N$-layer lattice with the number of layers equal to the number of regions. $v_{11}^{1}$ represents node $v_{11}$ at first layer while $v_{11}^{N}$ represents node $v_{11}$ at layer $N . w\left(v_{11}^{1}, v_{22}^{1}\right)=w\left(v_{11}, v_{22}\right)=w_{11,22}$ is the edge weight between nodes $v_{11}$ and $v_{22}$ at first layer which is proportional to the similarity of the nodes $v_{11}$ and $v_{22}$ in the input image $J$. The edge weights of the nodes between layers are set to zero to make the object layers disjoint, for example $w\left(v_{11}^{1}, v_{11}^{2}\right)=w\left(v_{11}^{1}, v_{22}^{N}\right)=0 \ldots \ldots \ldots$. . . . . . . . . . 37

3.2 Learning set pixels. . . . . . . . . . . . . . . . . . . . . . 43

3.3 Textures and synthetic images generated to evaluate multi-layer random walker image segmentation algorithm: a) textures [13] and b) image mixtures. . . . . . . .

3.4 Segmentation results for multi-layer user input random walker image segmentation method (first row), original RW [1] (second row), and the ground truth (third row). a) Image mixture with two sources, b) image mixture of three sources. The seeds are selected by the user. . . . . . . . . . . . . . . . .

3.5 Segmentation results for cervical images with input image with initial seeds (left), multi-layer user input random walker image segmentation method (right top), original RW [1] (right middle), and ground truth image segmentation (right bottom). The seeds are selected by the user. . . . . . . . . . . . . . .

3.6 The ground truth image with three translucent overlapped sources in the image mixture (first column). Initial seeds that are used for the second, third and last columns are shown as red points in the original image. Segmentation results for [1] and [3] with one seed for each segment (second and third columns, respectively), learning-based multi-layer random walker image segmentation method without seeds (fourth column), and learning-based multi-layer random walker image segmentation method with one seed for each segment (last column). . . . . . . . . . . 52 
3.7 Segmentation results for cervical images using multi-layer multi-label random walker image segmentation method. The original synthetic cervical cell (first column), proposed multi-layer user input RW image segmentation method (second column), proposed multi-layer multi-label RW image segmentation (third and fourth column), and ground truth image segmentation (last column). The seeds are put by user. . . . . . . . . . . . . . . . . . 5

4.1 Proposed network with residual blocks for image segmentation using translucent partially overlapped objects. . . . . . . . . . . . . . . . . . 59

4.2 An overview of our proposed separation network with $S C_{1, i}, i=1, \ldots, 6$ and $S C_{2, i}, i=1, \ldots, 3$ representing the skip connections within and between encoder and decoder blocks respectively, $E_{i}, D_{i}, i=1, \ldots, 4$ the four encoder and decoder convolutional blocks, and In and Out are the input and output images. Solid arrows represent the normal layer connections while dotted arrows represent the skip

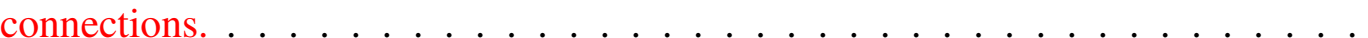

4.3 A few synthetic images and corresponding labels for the first dataset. The absence of an object is presented with a black object map. . . . . . . . . . . . . . . 62

4.4 A synthetic cytology image and its corresponding labels for the second dataset. . . 64

4.5 A few synthetic image mixtures of partially overlapped two cervical cells using Beer-Lambert law $[14] \ldots \ldots \ldots \ldots \ldots \ldots$. . . . . . . . . . . . . . . . . . . .

4.6 Results for the proposed network on first dataset. Top: segmentation results, middle: ground truth segmentation, bottom: original image mixture and image sources.

4.7 Results for the proposed network on our second dataset. Left: original image mixture, middle: segmentation results, right: ground truth. . . . . . . . . . . . . 68

4.8 Results for the proposed network on third dataset. Left: original image mixture, top right: ground truth segmentation, bottom right: segmentation results. . . . . . . 69 
5.1 Proposed three-step image segmentation method for jointly overlapped cells dataset. The confident pixels in third step are pixels with high probability values from CNN probabilistic output maps. . . . . . . . . . . . . . . 72

5.2 Proposed three-step image segmentation method for multiple overlapped cells dataset. 73

5.3 Proposed residual CNN for initial probabilistic image segmentation that produces multi-layer probabilistic image maps (first step of Fig. 5.1, 5.2) . . . . . . . . . 75

5.4 A training sample containing (a) the CNN input image and the binary training masks for the (b) background, (c) nuclei, and (d)-(e) two non-overlapping cytoplasm images. . . . . . . . . . . . . . . . . . 76

5.5 A training sample containing (a) the CNN input image and the binary training masks for the (b) background, (c) nuclei, and (d)-(g) four non-overlapping cytoplasm images. . . . . . . . . . . . . . . . . . . . . 77

5.6 A few samples of individual cervical cells from the ISBI 2014 Overlapping Cervical Cytology Image Segmentation Challenge dataset [15] to create jointly overlapped cervical cell dataset using the Beer-Lambert law [14] . . . . . . . . . . . 78

5.7 Illustration of the Beer-Lambert law for two overlapping cells [14] . . . . . . . . 79

5.8 A few synthetic image mixtures from jointly overlapped cervical cell dataset, created using Beer-Lambert law using (5.2) for $N_{o}(x) \leq 2 \ldots \ldots$. . . . . . . . 79

5.9 Sample images from the CNN and RW processing: (a) original image, (b)-(e) the CNN output probabilistic maps for (b) background $I_{b}$, (c) cell nuclei $I_{n}$, and the two cytoplasm layers (d) $I_{c_{1}}$, (e) $I_{c_{2}}$. (f) A cell's nucleus location, (g) confident pixels as seeds for cytoplasm (red) and background (green), (h)-(i) nuclei-seeded RW segmentation output and its corresponding probabilistic map. . . . . . . . . 83

5.10 Two examples of region growing for cytoplasm approximation. a),c) Original images, b),d) segmentation results. . . . . . . . . . . . . . . . 85 
5.11 Cell segmentation results after cytoplasm refinement algorithm for one overlapped region. a) Original image, b) CNN binary output result, c) region of interest for refinement, d)-e) first and second cell segmentation using Algorithm 7. . . . . . . .

5.12 Our proposed multi-layer random walker image segmentation method. a) input image, b) nuclei locations, c) cytoplasm approximate using Algorithm 6, d) cytoplasm refinement using Algorithm 7, and e) final segmentation using Algorithm 8. . . . .

5.13 Example experimental result for the proposed three-step CNN-based image segmentation method. a) input image, b) nuclei location extraction, c) nuclei-seeded region growing using Algorithm 6, d) cytoplasm candidates using thresholding, e) cytoplasm refinement using Algorithm 7, f) final cytoplasm segmentation using Algorithm 8 , and $\mathrm{g}$ ) is the ground truth. The information from cytoplasm candidates improved the final segmentation results. For cells that cytoplasm candidates were not useful, the results from nuclei-seeded region growing was used for the final segmentation results. For the segmentation in e) compared to the ground truth, the segmentation performance in $\mathrm{f}$ ) is $A c c=0.9996, D S C=0.9939, F N R=0.0042$, $T P R=0.9958$, and $F P R=0.0002 \ldots \ldots \ldots \ldots$

5.14 Comparing image segmentation results. a) is the ground truth image segmentation, b) our proposed three step method, compared to three c)-e) graph-based image segmentation methods [5-7], f) three-step CNN-based image segmentation method [4]. 92

5.15 A few samples of individual cervical cells from the ISBI2014 Overlapping Cervical Cytology Image Segmentation Challenge dataset [15] to create multiple overlapped cervical cell dataset using Beer-Lambert Law [14]. . . . . . . . . . . . . 97

5.16 Example synthetic image mixtures from the overlapped cervical cell dataset created using $(5.2) \ldots \ldots \ldots \ldots \ldots \ldots \ldots \ldots \ldots \ldots \ldots \ldots \ldots \ldots \ldots \ldots \ldots \ldots \ldots$ 
5.17 Approximating cells' cytoplasm with a)-f) the CNN output probabilistic maps for (a) background $I_{b}$, (b) cell nuclei $I_{n}$, and the four cytoplasm layers (c) $I_{c_{1}}$, (d) $I_{c_{2}}$, (e) $I_{c_{3}}$, (f) $I_{c_{4}}$. f)-i) proposed method to approximate the cytoplasm. g) original image, h) A cell's nucleus location, i) confident pixels as seeds for cytoplasm (red) and background (green), j)-k) nuclei-based RW segmentation output and its corresponding probabilistic map and 1) final segmentation for the sample cell. The results from j), k) and c)-f) are conditionally merged to find final segmentation at 1). 101

5.18 Visualization for correlation of $M_{n c, j}$ and $M_{n c, j} \vee M_{c, j}$ : a) high correlation, b) low

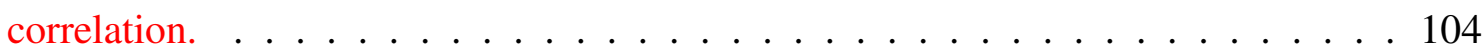

5.19 Cell segmentation results after cytoplasm refinement algorithm. a) Original image, b),f) nuclei-based cell cytoplasm, c),g) CNN-based cell cytoplasm, d),h) CNNbased cell refinement using Algorithm 7, e),i) final cell segmentation using Algo-

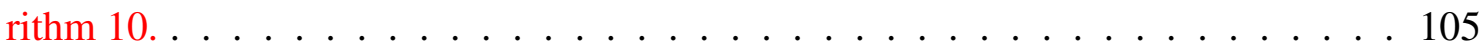

5.20 Our proposed multi-layer random walker image segmentation method. a) input image, b) nuclei locations, c) nuclei-seeded random walker image segmentation for region growing, d) segmentation results using region refinement and e) ground

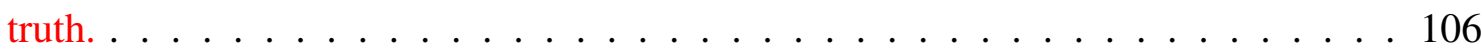


5.21 Example experimental result for the proposed three-step CNN-based image segmentation method. a) input image, b) nuclei location extraction, c) nuclei-seeded region growing using multi-layer random walker image segmentation, d) cytoplasm candidates using thresholding, e) cytoplasm refinement using Algorithm 7, f) final cytoplasm segmentation using Algorithm 10, and g) is the ground truth. The information from cytoplasm candidates improved the final segmentation results. For cells that cytoplasm candidates were not useful, the results from nuclei-seeded region growing was used for the final segmentation results. For the segmentation in e) compared to the ground truth, the segmentation performance in f) is $A c c=0.998$, $D S C=0.968, F N R=0.021, T P R=0.979$, and $F P R=0.001 . \quad \ldots \ldots 106$

5.22 Comparing image segmentation results. a) is the ground truth image segmentation, b) the proposed three stage method, compared to three c)-e) graph-based image segmentation methods [5-7], f) three-step CNN-based image segmentation method [4]. 107

5.23 Comparing image segmentation results. a) The original image, b) our proposed three stage method, compared to three c)-e) graph-based image segmentation methods [5-7], f) three-step CNN-based image segmentation method [4] . . . . . . . . 110

5.24 Comparing image segmentation results. a) The original image, b) our proposed three stage method, compared to three c)-e) graph-based image segmentation methods [5-7], f) three-step CNN-based image segmentation method [4] . . . . . . . 111

5.25 Comparing image segmentation results. a) The original image, b) our proposed three stage method, compared to three c)-e) graph-based image segmentation methods [5-7], f) three-step CNN-based image segmentation method [4]. . . . . . . . . 112

5.26 Comparing image segmentation results. a) The original image, b) our proposed three stage method, compared to three c)-e) graph-based image segmentation methods [5-7], f) three-step CNN-based image segmentation method [4]. . . . . . . . . 113 
6.1 Proposed two-stage CNN . . . . . . . . . . . . . . . . . . . . . . . . 117

6.2 Proposed residual network for image separation. . . . . . . . . . . . . . . . . 119

6.3 A few synthetic image mixtures of partially overlapped two cervical cells using Beer-Lambert law (5.2) . . . . . . . . . . . . . . . . . . . . . . 121

6.4 Results for the segmentation CNN. a) Original image, b) ground truth segmentation, c) segmentation results. . . . . . . . . . . . . . . . . . . . . . 122

6.5 Results for the separation CNN. a) Original image, b) ground truth original sources, c) separation results. The PSNR and SSIM values are averaged over the two decomposed images. . . . . . . . . . . . . . . . . . . . . . . . . . . 123

6.6 Good image segmentation leads to good image separation and small residual image. a) Ground truth image segmentation, b) ground truth image sources, c) image segmentation results, d) image separation results, e) original image, f) regenerated image, g) and h) residual image in linear and $\mathrm{dB}$ scales respectively. . . . . . . . 125

6.7 Poor image separation and large residual image as a result of poor image segmentation. a) Ground truth image segmentation, b) ground truth image sources, c) image segmentation results, d) image separation results, e) original image, f) regenerated image, g) and h) residual image in linear and dB scales respectively. . . . . . . . . 126

6.8 A few synthetic image mixtures of jointly overlapped cervical cells using BeerLambert law (5.2). . . . . . . . . . . . . . . 128

6.9 Results for our proposed two-stage method. a) Original image, b) ground truth image segmentation, c) ground truth image sources, d) image segmentation results, e) image separation results. The Acc, IoU, PSNR, and SSIM values are averaged over the 10 separated cells in the image mixture. Cells are shown in two nonoverlapped images for simplicity. . . . . . . . . . . . . . . . . . . . . . . . . 129 
6.10 Results for our proposed two-stage method. a) Ground truth image segmentation, b) ground truth image sources, c) image segmentation results, d) image separation results, e) original image, f) regenerated image, g) and h) residual image in linear and $\mathrm{dB}$ scales respectively. The Acc, IoU, PSNR, and SSIM values are averaged over the 10 separated cells in the image mixture. Cells are shown in two nonoverlapped images for simplicity. . . . . . . . . . . . . . . . 130

D.1 Gradient descent oscillates towards local optimum when state space is curved much more steeply towards one dimension. Left: stochastic gradient descent without momentum, right: with momentum [16] . . . . . . . . . . . . . . 177

D.2 Stochastic gradient descent methods with momentum. Left: original Stochastic gradient descent with momentum, right: Nestrov accelerated gradient method [17]. 178

D.3 Comparing Adagrad optimization method with Stochastic gradient descent method [18] . . . . . . . . . . . . . . . . . . . 179

D.4 RMSprop optimization method [19] . . . . . . . . . . . . . . . 180

D.5 Comparison between adaptive learning rate methods $[20] \ldots \ldots$. . . . . . 181

D.6 Different gradient optimization methods $[21] \ldots \ldots$. . . . . . . . 182 


\section{Abbreviations}

\section{List of Abbreviations}

\begin{tabular}{cc|cc}
\hline RW & Random walker & CPU & Central processing unit \\
CNN & Convolutional neural network & HE & Hematoxylin and eosin \\
MRF & Markov random field & Acc & Accuracy \\
GMM & Gaussian mixture model & IoU & Intersection of union \\
CRF & Conditional random field & SSIM & Structural similarity \\
SVM & Support vector machine & PSNR & Peak signal to noise ratio \\
Conv & Convolutional layer & PCA & Principal component analysis \\
FC & Fully connected layer & ICA & Independent Component Analysis \\
Relu & Rectified linear unit & SVD & Singular Value Decomposition \\
BN & Batch normalization layer & NMF & Non-negative matrix factorization \\
\hline & Graphical processing unit & & \\
\hline
\end{tabular}




\section{Chapter 1}

\section{Introduction}

Cervical cancer is one of the dangerous cancers in women around the world. Early detection of cancerous cells helps to improve the chance of treatment and some tests such as pap smear or Thinprep are important routine tests in screening to diagnose cervical cancer. Although cervical cell segmentation is a strong tool to detect abnormality of the nucleus and cytoplasm, it is difficult due to partial overlap of the cells. Also having the image values of each individual cervical cell in a multiple cell image give more information in a computer based diagnostic system but it needs cervical cell decomposition.

\subsection{Image Decomposition}

Image separation or image decomposition is to reconstruct image sources from the input image. Each image source corresponds to a physical object. Multiple image sources may combine linearly or non-linearly to create an image mixture. Let there be $m$ different image sources with size $a \times b$ and $n$ different mixing vectors of size $m \times 1$ creating $n$ different image mixtures with size $a \times b$. If the number of image mixtures which is called observations is more or equal than the number of image sources, there is a mathematical solution that can be achieved through different methods for image separation problem. 
However, if the number of observations is less than the number of image sources, the problem is called under-determined and there is not a guaranteed mathematical solution for these types of problems. One solution to overcome the lack of information is to use statistical information about the image sources that are going to be extracted. Image segmentation, partitioning an image into multiple sets of pixels with similar characteristics, can be considered as a side information for image decomposition. For example, in image matting problem a binary mask to show the location of foreground and background called alpha matt is given as side information to distinguish foreground from background.

The goal in this work is to perform image separation of image structures and objects that overlap in the imaging plane but exhibit transparency that could potentially be separated. Examples of imaging where object lucency commonly occurs includes cell cytology imaging, $\mathrm{x}$ ray imaging, positron emission tomography imaging, hyperspectral imaging, photographing semireflective/semi-transparent materials, and document scanning. In this dissertation, we focus on the problem of separating overlapping cells in cervical cell cytology images.

Four different common approaches for image separation are PCA, ICA, SVD, and NMF [22]. PCA projects the high-dimensional data into orthogonal bases that are second-order decorrelated. ICA calculates a transformation over the data to make them higher-order statistically independent. SVD projects the data into the maximum power bases calculated from the data. However, none of them guarantee to have non-negative elements, and therefore, the obtained source signals might not have physical meaning [22].

$\mathrm{NMF}$ is a strong tool that is being used in matrix decomposition where the factor matrices are nonnegative [23]. NMF is formulated as

$$
\mathbf{Y}=\mathbf{A X}
$$

where $\mathbf{Y}$ is the observation matrix and $\mathbf{A}$ and $\mathbf{X}$ are factors. However, since the decomposition problem is not convex, there will be no promise to find a unique solution to the problem. One way 
to overcome this non-uniqueness issue is to add more terms as constraints to the cost function in order to penalize unwanted solutions.

The main issue in these four approaches is that usually need the number of observations to be equal to or greater than the number of image sources. However, in some applications we need to separate a single image into two or more image sources and therefore, the above methods will not guarantee to give good results for these under-determined problems.

One solution for under-determined image separation problems is to use statistical information about the dataset that is going to be separated. For example, image segmentation can be considered as side information to solve under-determined image decomposition problems.

\subsubsection{Image Segmentation}

The goal in image segmentation is to divide an image into regions with similar characteristics. This process is widely used in different applications.

Image segmentation methods can be categorized into two main groups: supervised and unsupervised.

- Supervised methods try to find a model for segmentation first by focusing on the important features extracted from the image. These features can be extracted from the whole image or part of it.

A large subset of supervised methods that are widely used for image processing applications are machine learning methods [24]. A basic and fundamental step in common machine learning algorithms is extracting useful features from data. However, it is not always easy to find a good set of features for a specific type of data and application and finding a good set of features is still an open research area.

Deep neural networks in deep learning [25-27] have shown to solve this problem with the cost of high training time. Deep neural networks use complex structures and are trained on 
a large number of training data. Given enough training, these structures automatically find important features and learn based on these extracted features. Deep learning has emerged as a new field of research in machine learning since 2006 and has flourished in the past decade as a powerful tool in many learning applications, such as image segmentation.

One class of deep neural networks is convolutional neural networks (CNN) [28, 29]. CNNs usually use a feed-forward neural network structure because of simplicity [30-33]. CNNs typically consist of convolutional layers, pooling layers, fully connected layers and normalization layers [12,30-34]. These networks use gradient based back-propagation methods for weight tuning. CNNs have been used widely in image processing fields such as computer vision, medical diagnosis, and robot remote controls [35, 36].

- Unsupervised image segmentation methods rely on the image content and try to partition the image based on the similarity and discontinuity of the pixels [37]. Since unsupervised methods do not need manually-labeled data, these methods can be used for specific types of the applications where there are no labeled data.

- Graph-based approaches, which can be implemented as a subset of either supervised or unsupervised methods, represent the image as a graph with the pixels as graph nodes and similarity between pixels as graph edges. The similarity measure is a mathematical function that quantifies the similarity between two things by using their features. These features can be represented as the image intensity, color or edge information and will be defined based on the application. Graph-based approaches have been considered as powerful methods in image segmentation since 1971 [38].

Ordinary image segmentation methods are designed to create non-overlapping regions in images. But, in some data, such as cytology images or x-ray images, translucent regions partially overlap. Ordinary image segmentation methods will perform poorly or not work at all on data with 

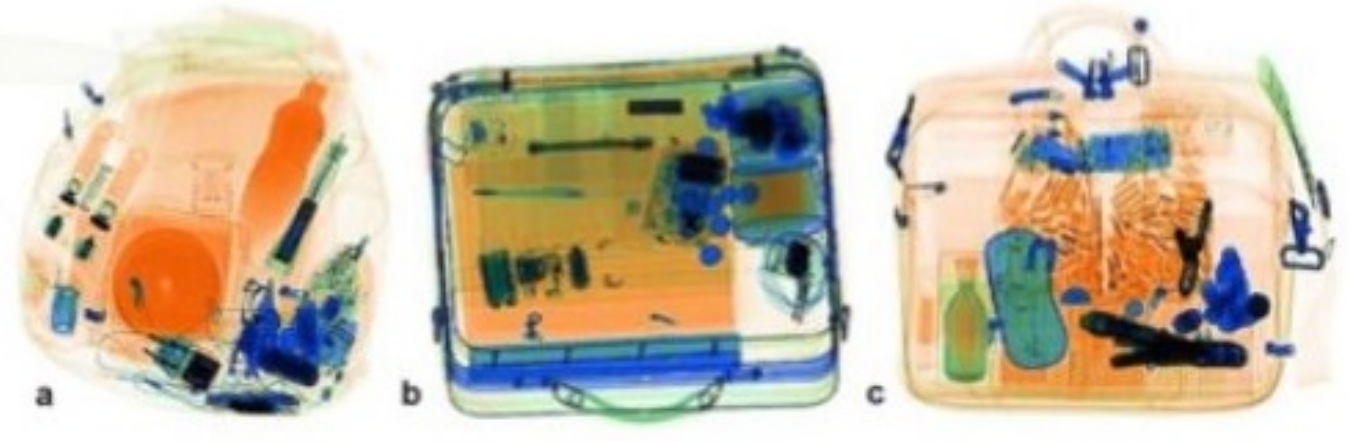

Figure 1.1: X-ray image samples from airport security devices [8].

overlapped regions.

One example of images with translucent partially overlapped objects are x-ray images. Having a set of partially overlapped objects, as x-ray radiation penetrates through the objects, the overlapped part which is denser than non-overlapped parts absorb more radiation. This results in an image with translucent partially overlapped objects. Figure 1.1 shows an example of pseudo colored xray imaging from airport baggage security.

Another example of images with translucent partially overlapped objects is cytology imaging where cells are usually stained before put under the microscope. The result of putting cells on slides causes pressure on them and makes a flat $2 D$ plane. Sometimes translucent parts of the cells partially overlap, which creates an image of combination of translucent partially overlapped objects (Figure 1.2).

Assume to have a mixture of two partially overlapped sources. Ordinary image segmentation methods can produce three different results. Either assign the overlapped region to first region or second region or make three regions, where none of them is the desired result. Therefore, the ordinary image segmentation methods do not give reasonable results for images with partially overlapped sources. One solution is to use multi-layer image segmentation, where it creates multiple 


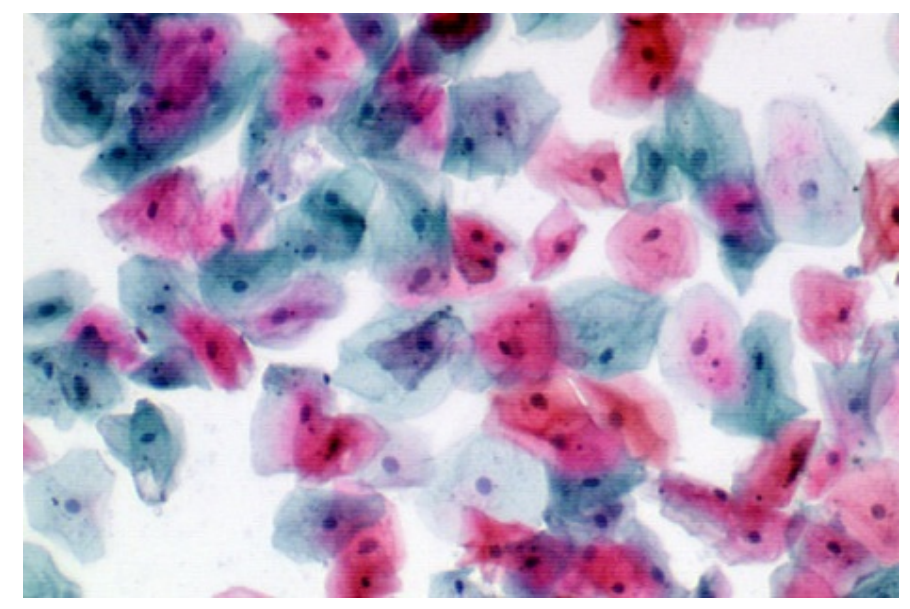

Figure 1.2: Papanicolaou (PAP) red stain kit (cytology stain) [9].

images in the output, each image having segmentation map of one of the sources. Multi-layer object detection of overlapped objects has recently received interest in different applications, such as airport baggage surveillance and health screening [39].

In this dissertation, we focus on overlapping cervical cytology images. Some research has been done on overlapping cervical cell segmentation recently. Lu et. al. [5] improved joint optimization multiple level set functions for overlapping cervical cells. Authors in $[6,40]$ set up a two-step framework to segment nucleus and cytoplasm of multi-layer overlapping cervical cells. Ushizima et. al. [41] proposed a framework based on region merging, local thresholding, and voronoi diagram for overlapping cervical cell segmentation. their work won cervical cytology image segmentation challenge 2014. Authors in [42] proposed a three-step segmentation method by using superpixel partitioning and cell-wise contour refinement for overlapping cervical cells. In [4, 43] authors created a multi-step approach for overlapping cervical cell segmentation using CNN. They refined the results of $\mathrm{CNN}$ for accurate segmentation. Although $\mathrm{CNN}$ is good at generalizing the training, it is not very good at getting highly accurate edges at the current implementations for cervical cell segmentation $[4,43]$. Therefore, $\mathrm{CNN}$ is good to give a rough segmentation of the cells, and then we have to do edge refinement after. 
Random walker image segmentation method is good at region growing so it can be used as the refinement step to fine tune the $\mathrm{CNN}$ segmentation results. However, random walker is sensitive to initial setup, so if the seeds are not extracted correctly, the final segmentation results will be poor.

By using a combination of $\mathrm{CNN}$ and random walker image segmentation, we take the advantage of each part and remove the disadvantage of it. Different from other methods that use discrete segmentation results from $\mathrm{CNN}[4,43]$ for fine tuning, we use $\mathrm{CNN}$ probabilistic map to try to guide the random walker at the refinement step.

\subsection{Contribution}

The goal in this work is to perform image separation of overlapping cervical cell images. We propose a two-stage method for image separation.

In the first stage, we propose a three-step CNN-based multi-layer image segmentation method that creates a multi-layer segmentation map from the input image where each layer represents one of the image sources in the input image. Starting with a residual UNet shape CNN (Appendices A.1,A.2), we create a probabilistic map of the nuclei, cytoplasms, and background for the input image [44]. A multi-layer random walker image segmentation method [3,45] is then applied for nuclei-based region growing. The cytoplasm borders are then refined using nuclei-based extracted regions and CNN-based cytoplasm candidates [46].

We then use image segmentation results for image separation in the second stage. In the second stage we design a new residual network (Appendix A.3) and we use the image along with the segmentation information obtained by our first stage as the input to train our proposed image separation CNN $[47,48]$.

One drawback for CNNs is the need of a large number of training data. However, in our case the training dataset is limited. We focused on cervical cell cytology images and created synthetic images close to the real images by using Beer-Lambart law [14]. 
In summary, our contribution is listed as follows

- We modified the original random walker image segmentation algorithm [1] and multi-label random walker image segmentation algorithm [2] to segment translucent partially overlapped objects (Chapter 3).

- We created an $N$-layer Laplacian matrix, where $N$ is the number of objects in the input image.

- We modified the seeds and boundary conditions for each matrix layer to produce an N-layer output image.

- These contributions were incorporated into the following publications

* T. L. Mahyari and R. M. Dansereau, "Random walks for image segmentation containing translucent overlapped objects," in 2017 IEEE Global Conference on Signal and Information Processing (GlobalSIP), pp. 46-50, Nov 2017 [3].

* T. L. Mahyari and R. M. Dansereau, "Learning-based multilabel random walks for image segmentation containing translucent overlapped objects," in 2017 40th International Conference on Telecommunications and Signal Processing (TSP), pp. 610-614, July 2017 [45].

- We designed a proposed convolutional neural network (CNN) to segment translucent partially overlapped objects (Chapter 4). This contribution was incorporated into the following publication

- T. L. Mahyari and R. M. Dansereau, ”Deep learning methods for image segmentation containing translucent overlapped objects," in 2019 IEEE Global Conference on Signal and Information Processing (GlobalSIP), pp. 1-5, Nov 2019 [44]. 
- We proposed a three step multi-layer image segmentation method where we combined the designed $\mathrm{CNN}$ with the proposed random walker image segmentation method to improve cervical cell segmentation (Chapter 5).

- We used the probabilistic output maps from the designed CNN to guide our multi-layer random walker image segmentation algorithm.

- Using Hungarian algorithm [49], we matched the CNN-based segmentation results to the seeded-based segmentation results and modified them for fine tuning cell segmentation results.

- These contributions were incorporated into the following publication

* T. L. Mahyari and R. M. Dansereau, ”Two Step CNN-based method for Overlapped Cervical Cell Decomposition”, 2021. Submitted to Neurocomputing journal [48].

- We designed a new residual network (Appendix A.3) and we used the input image along with the segmentation information obtained by our first stage as the input to our proposed image separation CNN (Chapter 6).

- These contributions were incorporated into the following publications

- T. L. Mahyari and R. M. Dansereau, "Deep learning methods for image decomposition of cervical cells," Accepted in 28th European Signal Processing Conference (EUSIPCO 2020), 2020 [47].

- T. L. Mahyari and R. M. Dansereau, "Multi-layer Random Walker Image Segmentation for Overlapped Cervical Cells using Probabilistic Deep Learning Methods”, 2021. Ready to submit, journal paper [46].

- We created synthetic images close to the real images by using Beer-Lambart law [14] for our both designed CNNs to overcome the lack of large number of available labeled training data 
(Chapters 5, 6).

\subsection{Dissertation Preview}

In Chapter 2, we give background on graph-based and learning-based image segmentation methods. Then we explain machine learning methods, deep learning and convolutional neural network methods. In Chapter 3, we propose a multi-layer random walker image segmentation method for partially-overlapped translucent regions. Our proposed multi-layer image segmentation method using convolutional neural networks is explained in Chapter 4. Our three-step CNN-based algorithm for multiple overlapped cervical cell image segmentation is explaind in Chapter 5. Chapter 6 explains our proposed two-stage CNN for image decomposition. Finally, conclusions and future work comes in Chapter 7. 


\section{Chapter 2}

\section{Background}

In many image segmentation applications the objects in the image are not overlapped. However, for some data the objects in the image are partially overlapped.

\subsection{Partially Overlapped Translucent Objects}

Xray images and cytology images are two examples of the partially overlapped translucent objects. In the following, we explain these two types of images.

\subsubsection{X-ray Images}

$\mathrm{X}$-ray is a high frequency electromagnetic radiation with the typical wavelength of 0.01 to 10 nanometers first discovered by Wilhelm Conrad Röntgen in 1895 [50].

Because of the short length of waves, x-rays usually penetrate through materials and barely interact with them which results in sharp shadows for radiography. X-rays are categorized in two main classes of hard and soft. 
- Hard x-rays have higher radiation energies. These high energy photons are not easily absorbed and penetrate through the materials very easily. Because their high energy and momentum makes them difficult to stop, they are dangerous for living organisms and are usually used for non-living applications such as bagging inspections.

- Soft x-rays have lower radiation energy. This type of $\mathrm{x}$-ray is used for applications with thinner and more volatile objects. Also, because soft x-rays are less harmful, they are used for medical purposes.

As x-ray passes through an object, the denser parts or the object absorb more radiation and therefore, makes darker regions on the image. The amount of x-ray energy absorption depends on two factors:

1) The thickness of the material $x$

2) The characteristics of the material, called absorption coefficient $A$.

The energy absorption can be written as

$$
I=I_{0} e^{-\mu \rho x}
$$

where $I_{0}$ is the initial x-ray beam intensity, $\mu$ is linear attenuation coefficient, and $\rho$ and $x$ are the density and thickness of the material respectively.

Assume that two objects are overlapped partially. As the x-ray penetrates through the objects, the overlapped part which is denser than non-overlapped parts absorb more radiation. Assuming to have close density values for the two overlapped objects, we can formulate energy decay for the overlapped part as the linear superposition of the energy decay of the two individual objects. Figure 2.1 shows an example of x-ray for airport bagging inspection. 


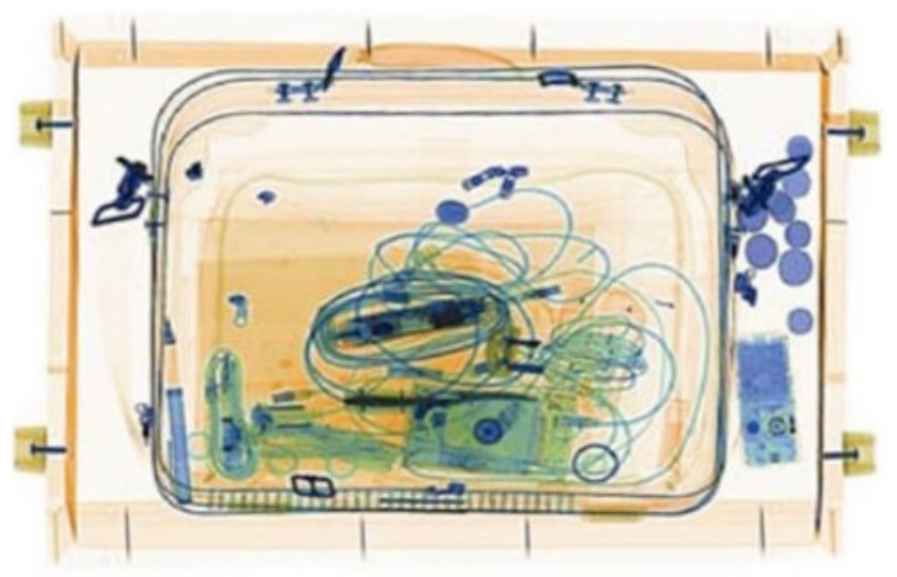

Figure 2.1: An xray image sample from the airport security device [10].

$\mathrm{X}$-ray imaging is used in many applications such as bagging inspection [51], medical imaging [52], astronomy [53], material analysis [54], manufacturing quality control [55], and fraud detection in art [56].

\subsubsection{Cytology Images}

Cytology is the examination of our body cells under microscope.

Although the science of cytology and cytopathology recognized in $18^{\text {th }}$ century, the standardization of this branch of pathology was not founded until late $20^{\text {th }}$ century [57]. The science of cytopathology has two major branches, exfoliative and aspiration biopsy [57].

- In exfoliative cytology, the samples are from exfoliated surfaces. This includes gynecological samples, respiratory/exfoliative cytology, urinary cytology, body fluid cytology, gastrointestinal tract, discharge cytology, scrape cytology [57].

- In aspiration biopsy a fine needle is used to make diagnosis on aspirating cellular material from any lesion in the body [57].

In cytology imaging, cells are usually stained before put under the microscope. There are 


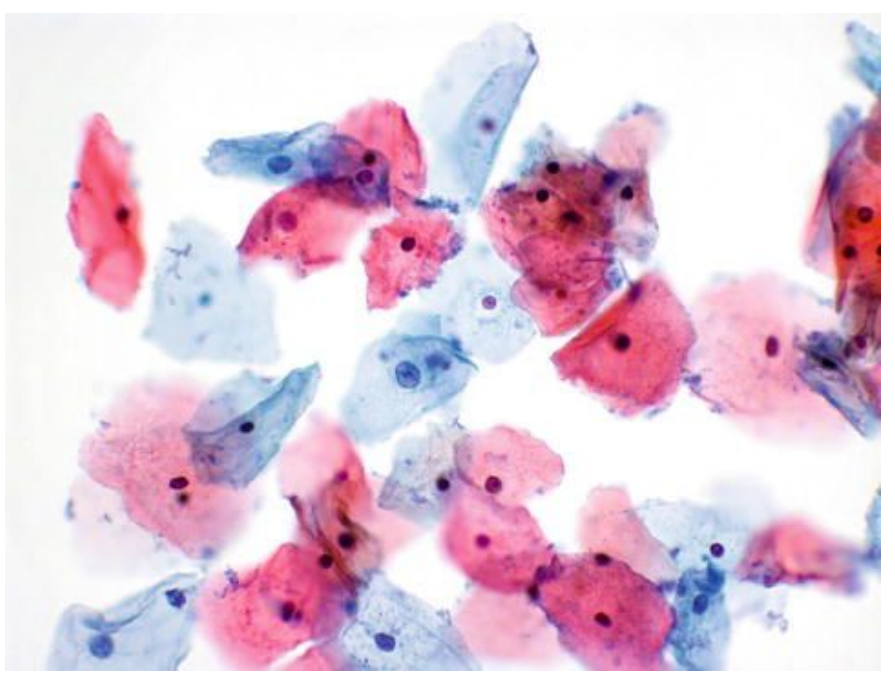

Figure 2.2: Normal pap. Superficial cells with smaller nuclei, and intermediate cells (center) with larger nuclei are seen [11].

three common ways of staining which are air-dried based stains, ethanol-based solution stains, and hematoxylin and eosin (HE) stains [57]. The result of putting cells on the slides causes pressure on them and makes a flat $2 D$ plane. However, sometimes cells partially overlap which creates an image of combination of translucent partially overlapped objects (Figure 2.2).

\subsection{Image Segmentation}

Image segmentation is widely used in different applications such as object detection [58], object recognition [59], and disease diagnosis in medical imaging [60, 61].

Graph-based approaches which can be implemented as a subset of either supervised or unsupervised methods represent the image as a graph with the pixels as graph nodes and similarity between the pixels as the graph edges. The similarity measure is a mathematical measure to calculate the similarity between two or more objects in the image. It can use image intensity, color or edge information as the measuring attribute and will be defined based on the application. Graph-based 
approaches have been considered as powerful methods in image segmentation since 1971 [38]. A method presented by Grady in 2006 [1] was a graph-based segmentation method to solve multidimensional image segmentation problems.

\subsubsection{Graph Based Image Segmentation Methods}

Graph-based segmentation methods model an image $I$ as a weighted undirected graph $G=\{V, \varepsilon\}$, where the graph nodes $V$ represent the image pixels and the edges $\varepsilon$ represent the neighborhood pixels. The edge weights are calculated by a similarity measure between the neighboring nodes. The algorithms then partition the graph by minimizing an energy function over the edge weights; nodes clustered to each partition are considered as a segment. Some examples are normalized cuts [62], random walker [1], graph cut [63], isoperimetric partitioning [64], and minimum spanning tree-based segmentation [65]. In [62], after generating the image graph, the authors partition the image nodes into disjoint sets of vertices where the similarity measure among the nodes in a set is high and across different sets is low. In [65], the author applied a minimal spanning tree on the edge weights to partition the nodes into a set of disjoint vertices. In [64], the authors change the segmentation problem into solving a linear system using the image graph. In [63], a max-flow/mincut analysis is used to find the minimum-weight cut between the source and the sink after providing some initial seeds.

\subsubsection{Markov Random Field}

Markov random field (MRF) is a graph were input images are modeled as random variables with relationships captured by the conditional dependencies [66] as graph edges. The outputs, or segments in the segmentation algorithm, can be predicted by a maximum a posteriori decision rule using Bayes' theorem. 
Supervised MRF image segmentation methods combine the concept of graph theory and learning systems by minimizing an energy function over the similarity of the nodes' features and the regularization of the neighboring pixels using initial user defined seeds.

Assume we have a set of nodes $V$ in an image graph and a set of labels $\mathcal{L}=\left\{\ell_{1}, \ell_{2}, \ldots, \ell_{Q}\right\} \in \mathbb{N}$ where each label $\ell_{k}$ represents a segment in the image and $Q=|\mathcal{L}|$ represents the cardinality of the set $\mathcal{L}$. The goal is assigning a label $\ell_{k}$ to each node $v, \mathcal{X}=\left\{\mathcal{X}_{v} \mid \mathcal{X}_{v}=\ell_{k}, v \in V, k=1,2, \ldots, Q\right\}$ relative to its similarity to that node.

It is shown in Appendix (B) that the solution is obtained by minimizing

$$
E(\mathcal{X})=\sum_{v_{i} \in V}\left\{\Phi_{v_{i}}\left(\mathcal{X}_{v_{i}}\right)+\alpha \sum_{v_{j} \in \mathcal{E}_{v_{i}}} \Psi_{v_{i} v_{j}}\left(\mathcal{X}_{v_{i}}, \mathcal{X}_{v_{j}}\right)\right\}
$$

where $\Phi_{v_{i}}\left(\mathcal{X}_{v_{i}}\right)$ is the data-term that calculates the similarity of node $v_{i}$ to label $\mathcal{X}_{v_{i}}, \Psi_{v_{i} v_{j}}\left(\mathcal{X}_{v_{i}}, \mathcal{X}_{v_{j}}\right)$ is the regularization term that penalizes if adjacent nodes $v_{i}$ and $v_{j}$ have different labels $\mathcal{X}_{v_{i}}$ and $\mathcal{X}_{v_{j}}$, and $\mathcal{E}_{v_{i}}$ is the neighbor set of node $v_{i}$. In other words, $\Phi_{v_{i}}\left(\mathcal{X}_{v_{i}}\right)$ finds the solution while $\Psi_{v_{i} v_{j}}\left(\mathcal{X}_{v_{i}}, \mathcal{X}_{v_{j}}\right)$ keeps the solution smooth over the neighborhood.

Different optimization algorithms have been employed to solve (2.2), such as graph cuts $[63,67]$ and random walker (RW) [1]. To help understand the random walker algorithm, we will define the notation for probabilistic image segmentation in next subsection. Then we will explain the original random walker image segmentation algorithm [1] before explaining our proposed method.

\subsubsection{Probabilistic Image Segmentation}

In image segmentation, a label from a labelset $\mathcal{L}$ is assigned to every node $v_{i}$ in the image graph and every label $\ell_{k}$ represents a segment. In probabilistic segmentation, a probabilistic unity-sum vector $\mathcal{P}=\left\{p_{1}, \ldots, p_{Q}\right\}$ is assigned to every node, where $p_{v_{i}}^{\ell_{k}}$ is the probability of assigning label $\ell_{k}$ to node $v_{i}$. One of the famous probabilistic image segmentation algorithms is random walker image segmentation algorithm first proposed by Grady [1]. 


\subsubsection{Random Walker Image Segmentation Algorithm}

The work by Grady was first to propose the use of RW in image segmentation [1]. Assume that we have an image graph $G=\{V, \mathcal{E}\}$ where the graph nodes $V$ represent the image pixels and the edges $\varepsilon$ represent the neighborhood pixels. Laplacian matrix $L$ is defined as

$$
L_{i j}= \begin{cases}d_{i} & \text { if } i=j \\ -w_{i j} & \text { if } v_{i} \text { and } v_{j} \text { are adjacent nodes } \\ 0 & \text { otherwise }\end{cases}
$$

where $w_{i j}$ is the edge weights between node $v_{i}$ and $v_{j}$

$$
w_{i j}=\exp \left(-\beta\left(J\left(v_{i}\right)-J\left(v_{j}\right)\right)\right),
$$

$d_{i}$ is the weighted degree of node $v_{i}$

$$
d_{i}=\sum_{j} w_{i j},
$$

where $J\left(v_{i}\right), J\left(v_{j}\right)$ are the intensity values of nodes $v_{i}$ and $v_{j}$ in the image graph $J(v)$ and $\beta$ is a free parameter. Also, assume that we have a set of all labeled nodes $M=\left\{m_{1}, m_{2}, \ldots, m_{T}\right\}$ called seeds where $T$ is the number of seeds and the set of all unseeded nodes $U=\left\{u_{1}, u_{2}, \ldots, u_{S}\right\}$, where $S$ is the number of unseeded nodes in the image graph.

By partitioning $V$ into $V_{M} \mid V_{U}$, such that $V_{M} \cup V_{U}=V$ and $V_{M} \cap V_{U}=\emptyset$, we can rearrange the Laplacian matrix $L$ to have seeded nodes $L_{M}$ and unseeded nodes $L_{U}$ partitioned as

$$
L=\left[\begin{array}{cc}
L_{M} & B \\
B^{T} & L_{U}
\end{array}\right] .
$$

Grady showed that minimizing (2.2) can be converted to solving the linear equation

$$
L_{U} p_{u_{i}}^{\ell_{k}}=-B^{T} p_{m_{i}}^{\ell_{k}}
$$


for one label or

$$
L_{U} \mathcal{P}_{U}=-B^{T} \mathcal{P}_{M}
$$

for all labels by using the combinatorial formulation of the Dirichlet problem [1], where matrices $\mathcal{P}_{U}$ and $\mathcal{P}_{M}$ are the probability of each label in $\mathcal{L}$ for all nodes $U$ and $M$ respectively:

$$
\begin{gathered}
\mathcal{P}_{U}=\left\{p_{u_{i}}^{\ell_{k}} \mid i=1, \ldots, S, k=1, \ldots, Q\right\}, \\
\mathcal{P}_{M}=\left\{p_{m_{i}}^{\ell_{k}} \mid i=1, \ldots, T, k=1, \ldots, Q\right\} .
\end{gathered}
$$

Having the set of $M$ seeds, Grady [1] then defined the boundary conditions as

$$
p_{m_{i}}^{\ell_{k}}\left\{\begin{array}{cl}
1 & \text { if } \mathcal{X}_{m_{i}}=\ell_{k} \\
0 & \text { otherwise }
\end{array}\right.
$$

where $p_{m_{i}}^{\ell_{k}}$ is the probability of assigning seed $m_{i}$ to label $\ell_{k}$. In other words, it assigns seed $m_{i}$ to its corresponding label $\ell_{k}$.

Solving (2.7) will give the probability $p_{u_{i}}^{\ell_{k}}$ of assigning label $\ell_{k}$ to node $u_{i}$ in the image graph.

Since the algorithm needs user input, it is not efficient for real time segmentation applications. Grady improved his work by adding learning information to the algorithm instead of user input [2].

For each label $\ell_{k} \in \mathcal{L},(2.2)$ can be represented as

$$
E_{\text {total }}^{k}=E_{\text {aspatial }}^{k}+\gamma E_{\text {spatial }}^{k}
$$

where

$$
E_{\text {aspatial }}^{k}=\sum_{v_{i} \in V} \Phi_{v_{i}}\left(\mathcal{X}_{v_{i}}=\ell_{k}\right)
$$

captures the similarity of nodes to label $\ell_{k}$ and

$$
E_{\text {spatial }}^{k}=\sum_{v_{i} \in V} \sum_{v_{j} \in \varepsilon_{v_{i}}} \Psi_{v_{i} v_{j}}\left(\mathcal{X}_{v_{i}}=\ell_{k}, \mathcal{X}_{v_{j}} \neq \ell_{k}\right)
$$


regularizes label's assignment around neighboring nodes. The goal is then to minimize the energy function $E_{\text {total }}^{k}$ in (2.12).

Grady [2] has shown that the minimum energy to (2.12) is obtained when $P^{k}$ satisfies the solution to

$$
\left(L+\gamma \sum_{r=1}^{Q} \Lambda^{r}\right) P^{k}=\lambda^{k}
$$

with

$$
\begin{gathered}
\Lambda^{k}=\operatorname{diag}\left(\lambda^{k}\right), \\
\lambda_{i}^{k}=p\left(\mathcal{X}_{v_{i}}=\ell_{k}\right),
\end{gathered}
$$

where $\lambda_{i}^{k}$ shows the probability density that the intensity at node $v_{i}$ belongs to the intensity distribution of label $\ell_{k}$ and $\lambda^{k}$ is the vector of all nodes' probabilities to label $\ell_{k}$. Therefore, in (2.15)

$$
P^{k}=\left\{P_{v_{i}}^{k} \mid v_{i} \in V\right\}
$$

where $P_{v_{i}}^{k}$ is the probability of assigning label $\ell_{k}$ to $v_{i}$ proportional to its intensity value and its neighborhood regularization.

Grady used intensity values as image features and image intensity differences as similarity measure for the data-term. However, in some applications higher dimensional image features and more complex similarity measures might be used to improve the results of the algorithm. We explained some of common similarity measures in Appendix C. 


\subsection{Machine Learning, Deep Learning and Convolutional Neu- ral Networks}

Machine learning is built upon the combination of different fields of science and mathematics such as statistics, neural networks, optimization theory, and adaptive control. Machine learning has been used on a variety of problems, including recognition, classification, and diagnosis where the decisions are supposed to be made by computer based on the learning phase [68].

A small branch of machine learning methods which is widely used recently and got real interest of researchers is deep learning (also called as deep structured learning or hierarchical learning) [35, 36].

\subsubsection{Deep Learning}

Deep learning can be described as four different related definitions [69]:

- A branch of machine learning methods that uses many non-linear internal (or hidden) layers for feature extraction, pattern analysis or classification. These methods can be either supervised or unsupervised.

- A branch of machine learning algorithms that uses hierarchical structure of layers to be able to represent the complex relationship among data in multiple layers of lower-level data representation. Therefore, they create a hierarchical model of features or concepts that high-level concepts will be represented through cascading the lower-level concepts.

- A branch of machine learning algorithms that make different layers of abstraction in a hierarchy model using artificial neural networks. the statistical lower-level layers will represent more complex higher-level statistical layers and concepts. 
- A branch of machine learning to make artificial intelligence closer to human intelligence by using multiple cascaded layers of representation and abstraction.

Common machine learning methods such as Gaussian mixture models (GMM), conditional random fields (CRFs), support vector machines (SVM), logistic regression models, and kernel regression models use at most two layers of non-linear feature transformation while deep learning methods have many-layer structures [69].

A basic and fundamental step in common machine learning algorithms is extracting useful features from data. However, it is not always easy to find a good set of features for a specific type of data and application and it is still an open research area. Deep neural networks in deep learning [25-27] have shown to solve this problem with the cost of high learning time. Using the idea of the structure of brain, deep neural networks [25-27] use complex structures and are fed a lot of information to train. Given enough training, these structures automatically find the important features and learn based on these extracted features.

\subsubsection{Convolutional Neural Networks}

One class of deep neural networks is convolutional neural networks, CNN, [28, 29]. CNNs usually use feed-forward neural network structure because of simplicity [30-33]. CNNs typically consist of convolutional layers, pooling layers, fully connected layers, relu layers, and normalization layers [12,30-34]. They use gradient based back-propagation methods for weight tuning.

- Convolutional layers (Conv) apply convolution operations to their input neurons of a layer and provide outputs to those neurons and pass them as input to the neurons of next layers. The output size of convolutional layers depend on filter size, strides and zeropads as well as the size of the input. For an input of size $W_{1} \times H_{1} \times D_{1}$ with $K$ filters of size $F \times F \times D_{1}$, 
with stride $S$ and zero pad $P$, the output size is $W_{2} \times H_{2} \times K$ where

$$
\begin{aligned}
& W_{2}=\frac{W_{1}-F+2 \times P}{S}+1 \\
& H_{2}=\frac{H_{1}-F+2 \times P}{S}+1
\end{aligned}
$$

with $F \times F \times D_{1} \times K$ weights and $K$ biases.

- Pooling layers combine a set of outputs of neurons from a layer to one output and pass it as input for the neurons of next layers. These layers typically use the maximum function to downsample the signal.

- Fully connected layers (FC) connect every neuron in one layer to every neuron in next layer.

- Rectified linear unit (Relu), is the most common used activation layer in CNNs because of its simplicity, fast convergence and sparsity characteristics. For the input value $x$, the output value of relu layer is $y=\max (0, x)$.

- Batch normalization layers $(\mathrm{BN})$ normalize the weights in layers.

\subsubsection{Optimization on CNNs}

After defining the network structure, the training then becomes an optimization problem to find the best weights of the nodes in the network to minimize the error between the obtained output and the desired output. Having weights $W$, the loss function is defined as $L(W, X, y)$, where $X$ and $y$ are the training examples and labels. The goal is then to find the best values for network parameters, weights, to minimize the loss function. Gradient Descent optimization algorithms are widely used in deep learning and specifically deep CNNs. Appendix D gives a list of common optimization methods that are used in CNNs.

CNNs have been used widely in image processing fields such as computer vision, medical diagnosis, and robot remote controls [35,36]. In the next section we first explain the structure of the 
most commonly used CNNs for image classification methods. We then explain the modifications made on the networks in order to be able to do semantic segmentation.

\subsection{CNN-based Image Segmentation}

The most common CNNs that are widely used for image classification are AlexNet [30], VGGNet [31], and GoogLeNet [32]. These networks use softmax function for image classification at the end. Assuming to feed an image $I$ to one of these networks, the input to the softmax layer becomes $x_{i}, i=1, \ldots, K$ vector, where $K$ is the total number of classes and $x_{i}$ represents the possibility of assigning class $i$ to the image. Softmax layer then creates output vector $y_{i}, i=1, \ldots, K$ as probability of assigning each class to that image

$$
y_{i}=\frac{e^{x_{i}}}{\sum_{j=1}^{K} e^{x_{i}}}, i=1, \ldots, K,
$$

In (2.20) $e^{x_{i}}$ changes all values to positive and dividing by $\sum_{j=1}^{K} e^{x_{i}}$ makes all values sum to one to create probability vector.

In this section we first describe the structure of these three famous networks as well as two other classification networks. Then we explain the modification made to the first three networks to use them for semantic segmentation.

\subsubsection{AlexNet, ZFNet, VGGNet, GoogleNet, and ResNet}

AlexNet was introduced in 2012 where the task was to classify images [30]. Authors in [30] created an 8 learned layer network and used gradient descent method on a batch of 128 examples to train their model. They trained their network 90 cycles through the training set of 1.2 million colored images of size $227 \times 227$ which took five to six days on two NVIDIA GTX 580 3GB GPUs. The model was tested then on ILSVRC-2010 dataset [70]. 


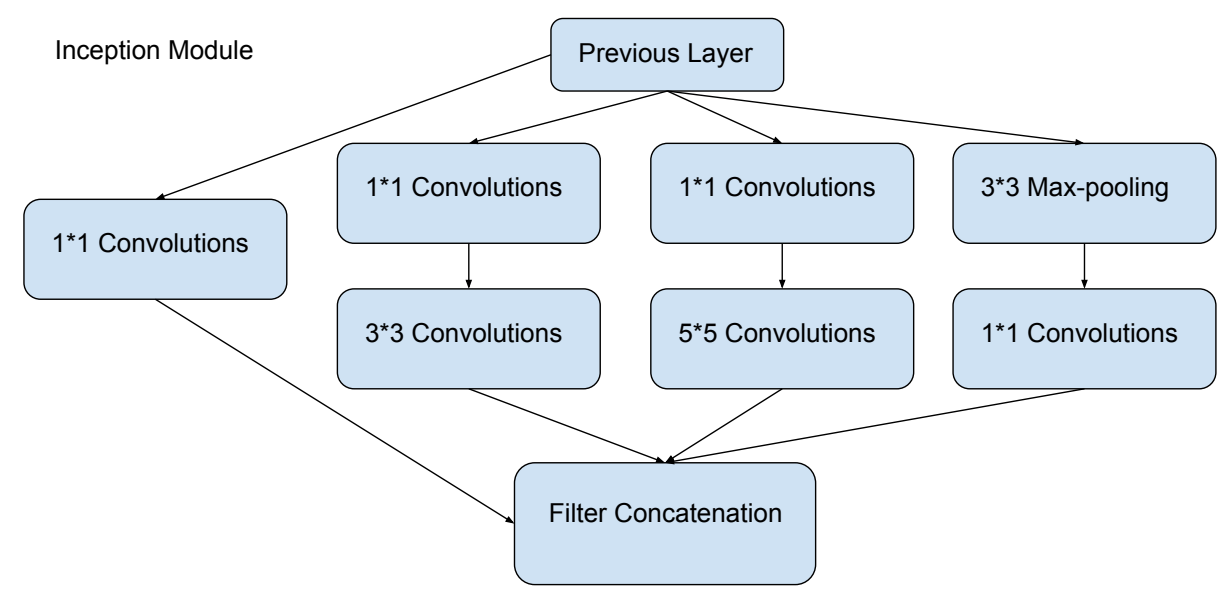

Figure 2.3: Inception layer for GoogLeNet.

In 2013 ZFNet [33] was introduced were authors used the same structure as in AlexNet but modified the network parameters. They trained their network through the training set of 1.3 million colored training images of size $224 \times 224$ with a set of 50 thousands and 100 thousands of validation and test images respectively.

In 2014 both GoogLeNet [32] and VGGNet [31] were introduced. Authors in VGGNet [31] used a $16-19$ layer network with convolutional layers of size $3 \times 3$. The model was applied on ILSVRC-2012 dataset [71] which includes images of 1000 classes, and is split into three sets: training (1.3 million images), validation (50 thousands images), and testing (100 thousandsimages with held-out class labels). On a system equipped with four NVIDIA Titan Black GPUs, training a single net took $2-3$ weeks.

Authors in GoogLeNet [32] implemented a 22 layers deep network by introducing the inception layer (Figure 2.3). They used the ILSVRC-2014 classification dataset [72] which involves the images of 1000 categories in the Imagenet hierarchy. This dataset split into three sets of 1.2 million images for training, 50,000 for validation and 100,000 images for testing. Their networks were trained using the DistBelief [73] on CPU based implementation; however, their model could be trained to convergence using few high-end GPUs within a week. 


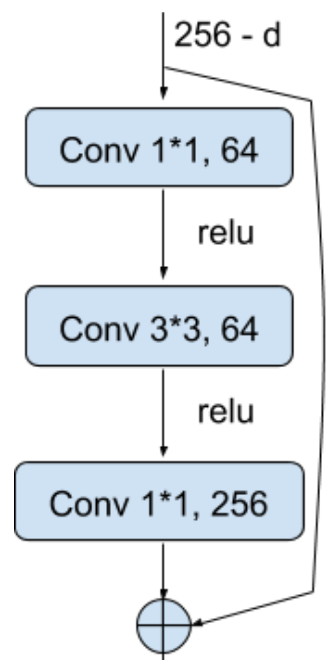

Figure 2.4: Learning residual function for ResNet.

In 2015 a new approach in creating the convolutional neural networks was introduced by [34]. This residual neural network called ResNet [34] allowed to create deeper networks with less complexity. By reformulating layers as learning residual functions, authors could gain accuracy from considerably increased depth (Figure 2.4). Comparing to VGGNet [31], ResNet [34] uses 8 times deeper network with less complexity.

To get more familiar with these networks, here we study their structures in details.

- AlexNet [30] contains eight learned layers: five convolutional and three fully-connected. The first convolutional layer filters the $227 \times 227 \times 3$ input image with 96 kernels of size $11 \times 11 \times 3$ with a stride of 4 pixels (this is the distance between the receptive field centers of neighboring neurons in a kernel map). The second convolutional layer takes as input the (response-normalized and pooled) output of the first convolutional layer and filters it with 256 kernels of size $5 \times 5 \times 48$.

The third, fourth, and fifth convolutional layers are connected to one another without any intervening pooling or normalization layers. The third convolutional layer has 384 kernels of size $3 \times 3 \times 256$ connected to the (normalized, pooled) outputs of the second convolutional 


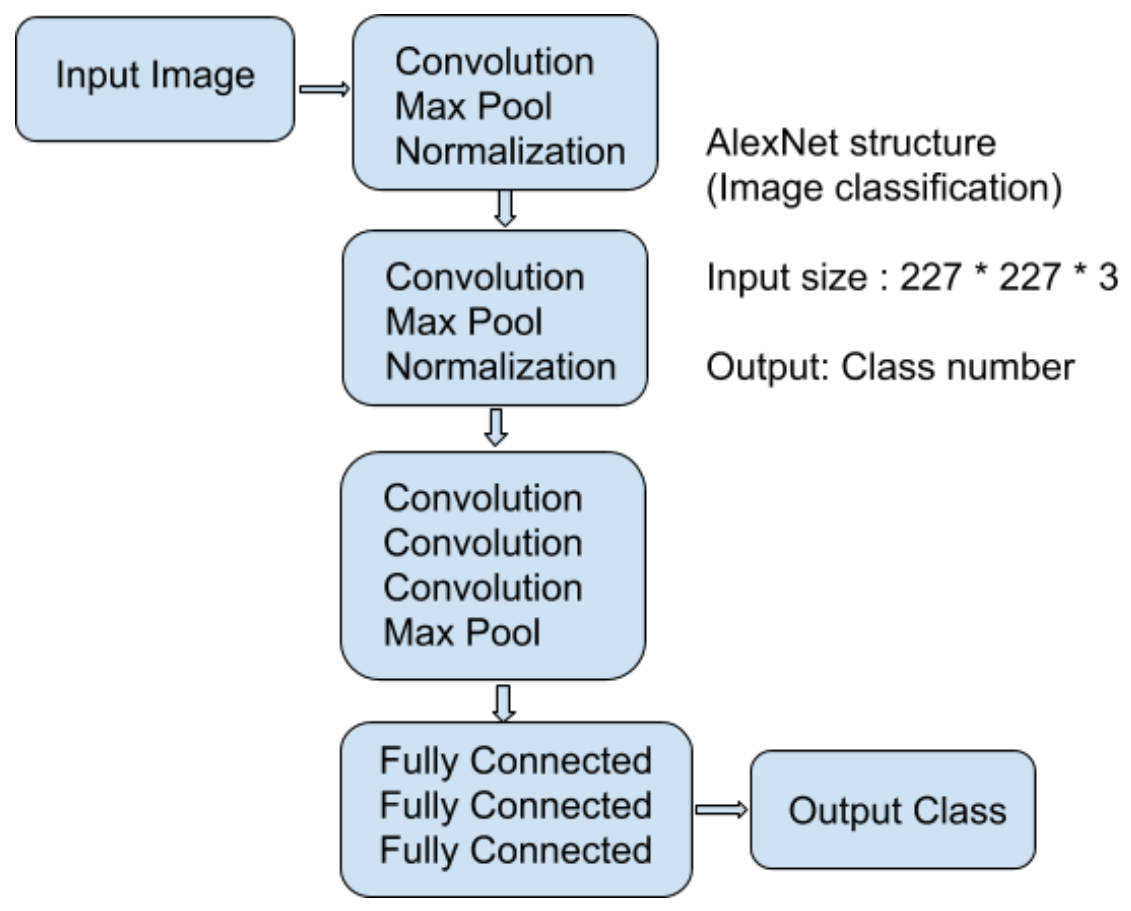

Figure 2.5: AlexNet structure.

layer. The fourth convolutional layer has 384 kernels of size $3 \times 3 \times 192$, and the fifth convolutional layer has 256 kernels of size $3 \times 3 \times 192$. The fully-connected layers have 4096 neurons each. Figure 2.5 shows the network structure.

- A colored image of size $224 \times 224$ is given to ZFNet [33] as an input. Similar to AlexNet [30] an 8 learned layer network is used to learn the model. However, the authors in [33] use kernels of size $7 \times 7 \times 3$ with a stride of 2 for the first convolutional layer. Also instead of $384,384,256$ number of filters for the third, fourth and fifth convolutional layers in AlexNet [30], authors in ZFNet [33] use 512, 1024, 512 number of filters respectively. Figure 2.6 shows the network structure.

- In VGGNet [31] the input of a fixed-size $224 \times 224$ RGB image will be fed to the network after subtracting the mean RGB value which is computed on the training set, from each pixel. 


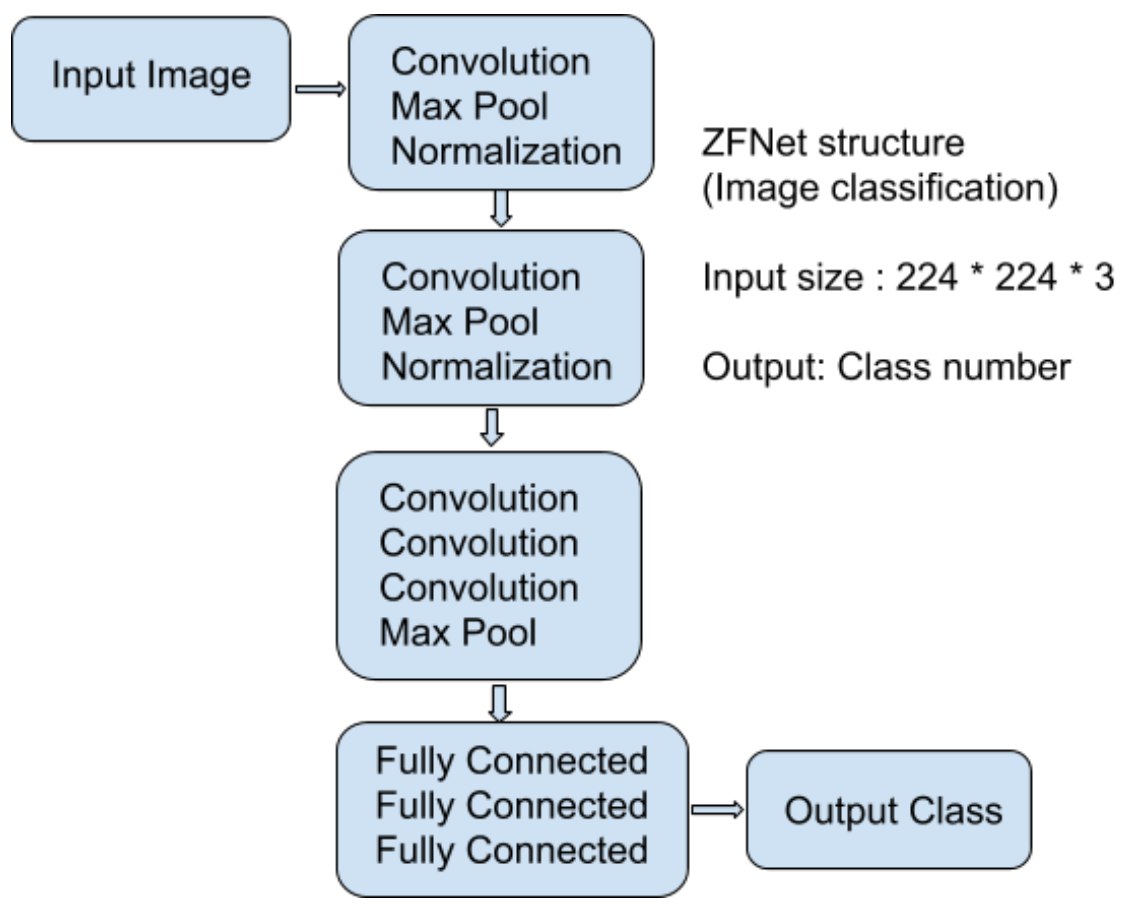

Figure 2.6: ZFNet structure. 
The size of convolutional layers is $3 \times 3$. In one of the configurations they also utilize $1 \times 1$ convolution filters, which can be seen as a linear transformation of the input channels (followed by non-linearity). The convolution stride is 1 pixel with the padding of 1 pixel for $3 \times 3$ convolutional layers. Five max-pooling layers follow some of the convolutional layers (not all the convolutional layers are followed by max-pooling). Max-pooling is performed over a $2 \times 2$ pixel window, with stride 2 . A stack of convolutional layers (which has a different depth in different architectures) is followed by three fully connected layers: the first two have 4096 channels each, the third performs 1000 neurons. The final layer is the soft-max classification layer. The configuration of the fully connected layers is the same in all networks. All hidden layers are equipped with ReLU [30]. Figure 2.7 shows the network structure.

- GoogLeNet [32] is a 22 layers deep learning network with two convolutional layers of size $7 \times 7$ with stride of 2 and $3 \times 3$ with stride of 1 respectively, three max pool layers of size $3 \times 3$ with stride 2 , one average pool layer of size $7 \times 7$ with stride 1,9 inception layers, one $40 \%$ dropout layer, one fully connected layer with 1000 neurons and finally, a soft-max layer. Figure 2.8 shows the network structure.

- ResNet [34] is a 152 layers deep learning network. A colored image of size $224 \times 224$ is fed to the network as input. Authors in [34] use Stochastic Gradient Descent (SGD) with a mini-batch size of 256. Their learning rate starts from 0.1 and is divided by 10 when the error plateaus. They do not use dropout layer. Figure 2.9 shows the network structure.

These structures were first implemented for image classification, however, in [74] the authors modified AlexNet, VGGNet and GoogLeNet for semantic segmentation. They simply added upsampling layers to the network to increase the size of the intermediate layers that were decreased after convolutional layers and max pool layers. In this way they kept the size of the output layer 


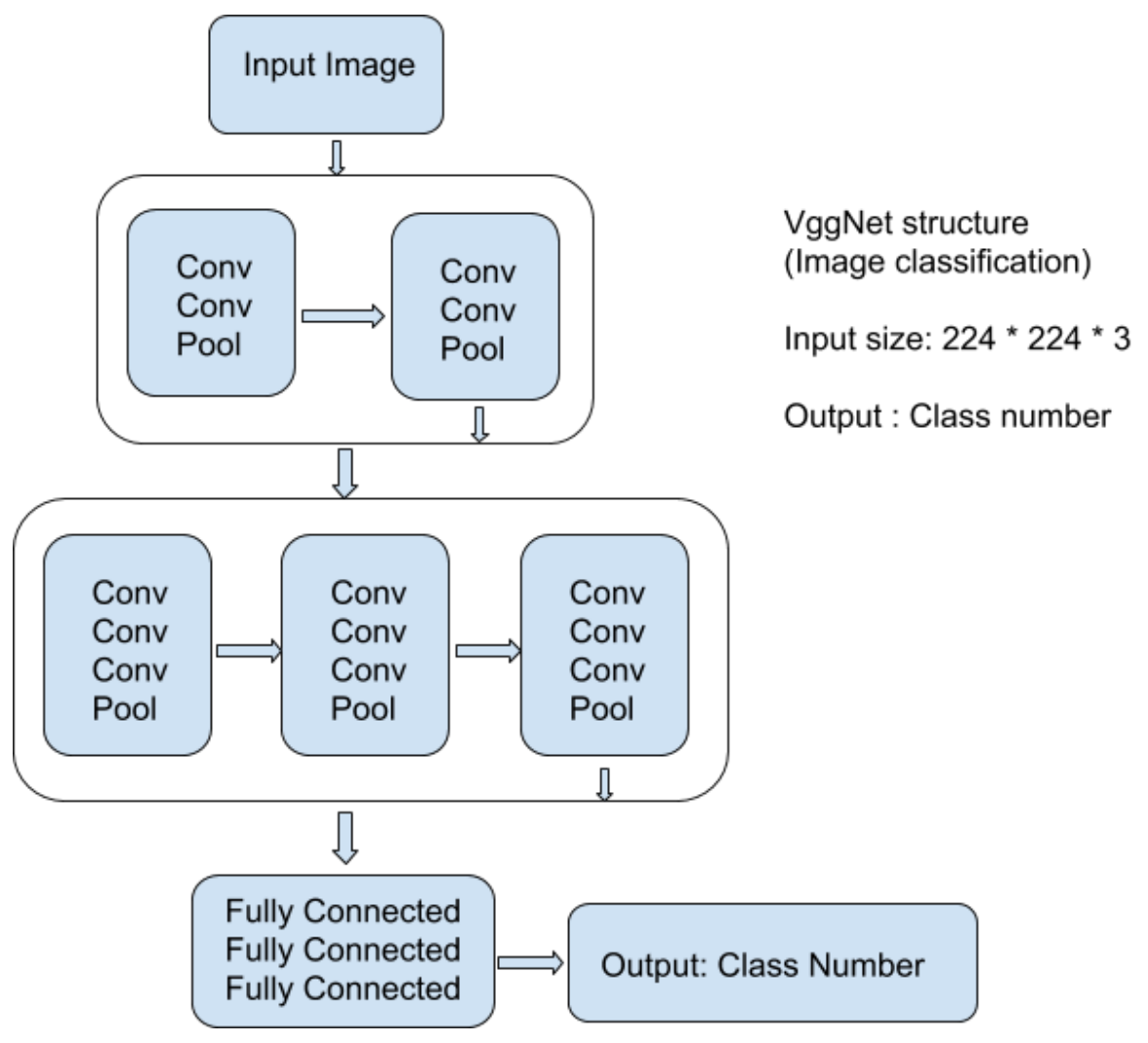

Figure 2.7: VGGNet structure. 




Figure 2.8: GoogLeNet structure.

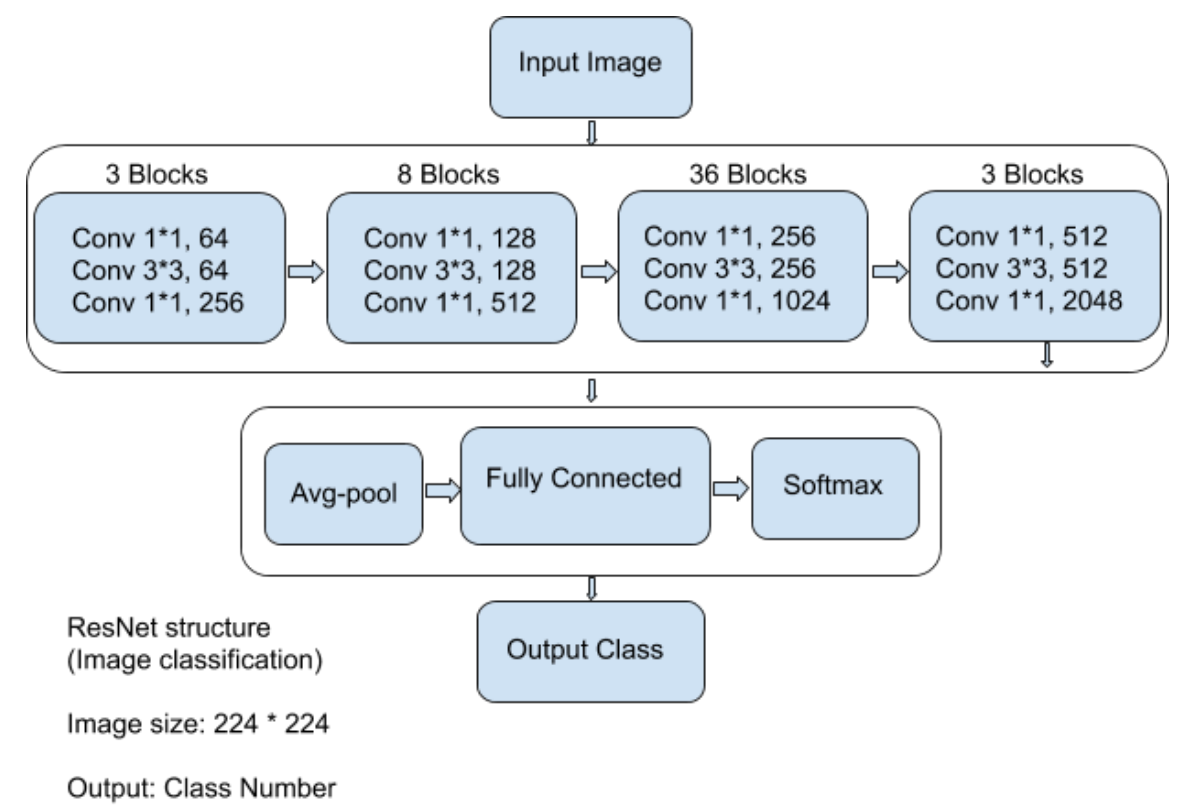

Figure 2.9: ResNet structure. 


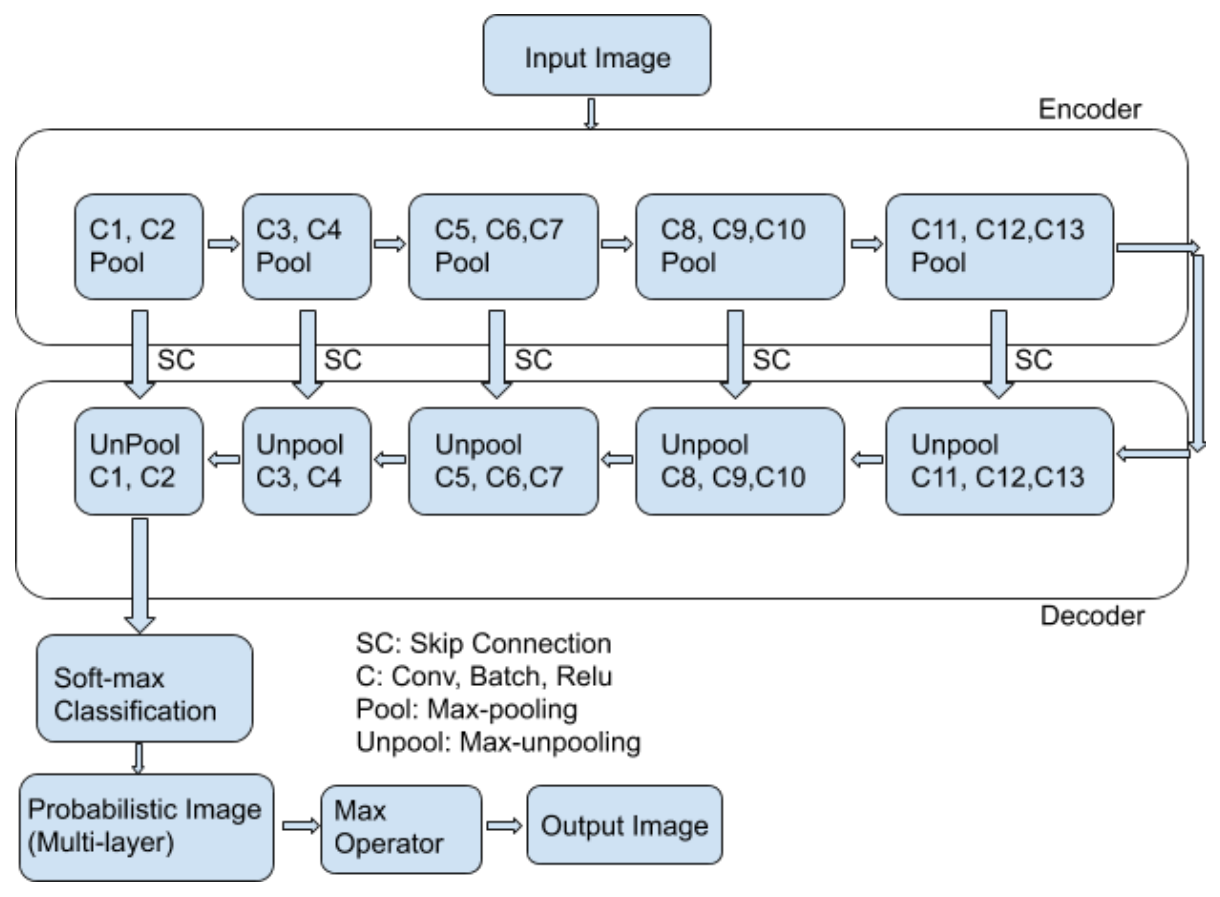

Figure 2.10: SegNet network [12] for semantic image segmentation.

the same as the size of the input image so that the learning could be done for segmentation. Following [74], authors in SegNet [12] modified VGGNet [31] to use transfer learning for image segmentation application (Figure 2.10).

These networks are trained with thousands of different types of image data to make a standard network for many applications, therefore, the number of layers and weights is large.

To do image segmentation using CNNs, there are two different ways:

- Implement one's own structure and tune the weights using the input/output learning phase. In this approach, the network will be application dependent and the number of layers and weights can be reduced because of the specific type of data that is going to be processed. However, since there are a large number of weights in a CNN, this approach needs a lot of time and data during training. Therefore, choosing this approach over transfer learning is 
dependent on the application [75].

- Start with a pre-trained network and augment the training with application specific data, which is called transfer learning. This approach is often preferred since one can save on the amount of data needed and the learning time.

\subsection{Image Decomposition}

A form of image mixtures occurs when imaging through glass or some semi-reflective/semitransparent medium that creates overlays of image information where the scene on the other side of the glass is mixed with a reflection on the glass. In [76], a joint diagonalization parameter estimation approach is taken for a frequency-domain separation. In [77], this type of reflection mixture is handled using a cyclic permutation approach while in [78], a generalized mixture ratio is estimated for the image separation. In [79], an iterative sparse blind separation algorithm is employed.

Other image separation techniques rely on multiple images or separate characteristics of the images. In [80], bleed-through in two-sided document scanning is considered where scans of either

side of a document reveal shared elements with different cross-mixture levels. In [81] and [82], hyperspectral image information is used to help separate image elements based on the different spectral characteristics of those image elements. In [83], the two different but correlated images captured from dual energy x-ray imaging are used when performing image separation. In [84], information from x-ray imaging and visible-light imaging of a painting are used to reveal and separate hidden paintings on canvases that have been painted over.

Many works have used NMF method for image decomposition [22,85-92]. Some works such as Zhou et al. [85] and Miao et al. [22] added the volume constraint term while others such as Qian et al. $[86,91]$ used sparsity as the constraint in the time and wavelet domain respectively. Some others used the graph regularization term as their constraint [87-90] and in some other works, 
authors used a transformation domain to solve NMF problem [92].

Although these methods decompose images into sources with physical meaning, these method usually need more than one observation to work properly. Therefore, these methods will not guarantee to give good results for under-determined problems. One way to solve under-determined image separation problem is to extract some statistical information about the image sources that are going to be extracted.

Some works use learning methods to extract these types of information. Authors in [93] used CNNs to extract foreground region from the background. They created 49300 training images containing 493 distinct foreground images and chose 49300 background images from a database of 100000 images. They also created a foreground mask for each of the training images. They designed a network and trained their network using the training images and their corresponding masks. They then used the trained network to decompose foreground region in a test image by giving the test image and its corresponding mask.

In this dissertation, we use segmentation map of partially overlapped cervical cells for image separation. Our proposed method is explained in the next Chapters.

\subsection{Summary}

Convolutional neural networks (CNNs) are recently used widely in computer vision applications, such as image segmentation. SegNet network [12] is a well-known CNN for semantic segmentation. Although SegNet has shown good performance over a wide variety of images, the complexity of the system is high because of a large number of network layers and parameters. Also SegNet is trained over images with non-overlapping regions. In many image segmentation applications the regions in the image are not overlapped. However, for some data the regions in the image are partially overlapped. Therefore, SegNet segmentation accuracy might not be excellent for images with translucent partially overlapped objects. Also, none of the above CNNs are used for image 
separation. To segment and separate partially overlapped regions using CNNs, one can implement a new network with fewer network layers and train it from the scratch for their specific applications. 


\section{Chapter 3}

\section{Multi-Layer Random Walker Image Segmentation}

\subsection{User-interactive Multi-Layer Image Segmentation}

We propose a method based on the random walker image segmentation algorithm to segment an input image into a set of output images, called layers, each containing one segment in that input image. Similar to the original random walker image segmentation method [1], a user will select a set of pixels, called seeds, to guide the segmentation algorithm (user-interactive).

Assume that we have an input image $J: \mathcal{R}^{2} \mapsto \mathcal{R}$ as a mixture of $N$ overlapped regions. The goal is to generate $N$ output images $O=\left\{O_{a} \mid a=1,2, \ldots, N\right\}, O_{a}: \mathcal{R}^{2} \mapsto \mathcal{R}$ from the input image $J$, each containing one region in the input image.

We first create an image graph $G=\{V, \mathcal{E}\}$ for the input image $J$ [1]. Then $N$ copies of this $2 D$ lattice will be generated and overlaid to make a $3 D$ lattice $G=\left\{V_{1}, V_{2}, \ldots, V_{N}, \hat{\mathcal{E}}\right\}$, where $V_{a}=\left\{v_{i} \mid v_{i} \in V\right\}$ is an $a^{t h}$ copy of the image graph with $a=1,2, \ldots, N$ and $\hat{\mathcal{E}}=\left\{\mathcal{E}_{i a, j b}\right\}$, where $\mathcal{E}_{i a, j b}$ is the connection between nodes $v_{i}$ in the $a^{t h}$ layer and $v_{j}$ in the $b^{t h}$ layer (Figure 3.1) with edge weights

$$
w\left(v_{i}^{a}, v_{j}^{b}\right)= \begin{cases}w\left(v_{i}, v_{j}\right)=w_{i j} & \text { if } a=b, \\ 0 & \text { otherwise }\end{cases}
$$


where $w_{i j}$ is obtained by (2.4)

In Figure 3.1, nodes within each layer, $v_{11}^{1}, v_{12}^{1}$ for example, are connected to their 8 neighbors with edge weights proportional to their similarity measure calculated by (3.1) and (2.4), for example $w\left(v_{i}^{a}, v_{j}^{a}\right)=w\left(v_{i}, v_{j}\right)=w_{i j}$ with $i=11, j=12, a=1$. Each node is connected to its $9 \times(N-$ 1) neighbors between layers in the $3 D$ lattice.However, because the input image is a mixture of $N$ objects with statistically independent shapes, locations and orientations, the image values for overlapping regions in the input image are statistically independent. Therefore, the overlaid layers should be disconnected and edge weights between layers should be set equal to zero to remove dependence between the sources. For example, nodes between layers such as $v_{11}^{1}, v_{12}^{2}$ are connected with edge weights equal to zero to make the layers independent of each other.

Similar to [3], we generate a new symmetric Laplacian matrix $\hat{L}$ in order to diffuse the image values within the layers and not between them using

$$
\hat{L}=\left[\begin{array}{ccccc}
L^{1} & \mathbf{0} & \mathbf{0} & \cdots & \mathbf{0} \\
\mathbf{0} & L^{2} & \mathbf{0} & \cdots & \mathbf{0} \\
\vdots & & & & \\
\mathbf{0} & \mathbf{0} & \mathbf{0} & \cdots & L^{N}
\end{array}\right],
$$

where $L^{l}, l=1,2, \ldots N$ is the Laplacian matrix at layer $l$ and is obtained as

$$
L_{i j}^{l}= \begin{cases}d_{i}^{l} & \text { if } i=j \\ -w_{i j}^{l} & \text { if } v_{i}^{l} \text { and } v_{j}^{l} \text { are adjacent nodes } \\ 0 & \text { otherwise }\end{cases}
$$

where $d_{i}^{l}$ is the weighted degree of node $v_{i}$ at layer $l$

$$
d_{i}^{l}=\sum_{j} w_{i j}^{l}
$$




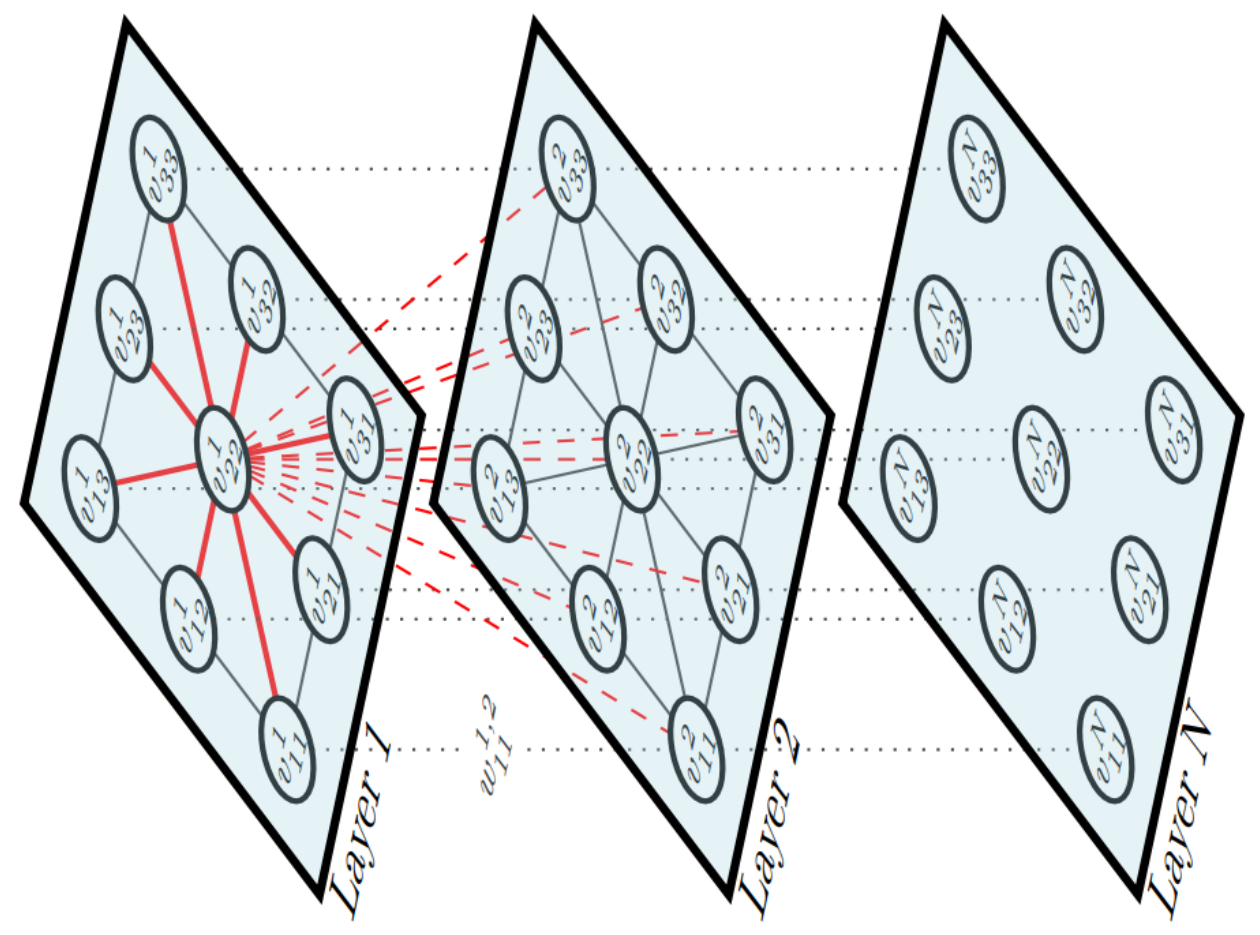

Figure 3.1: $3 D$ lattice structure for a $2 D$ image $J$ with $N$ regions. An $N$-layer lattice with the number of layers equal to the number of regions. $v_{11}^{1}$ represents node $v_{11}$ at first layer while $v_{11}^{N}$ represents node $v_{11}$ at layer $N . w\left(v_{11}^{1}, v_{22}^{1}\right)=w\left(v_{11}, v_{22}\right)=w_{11,22}$ is the edge weight between nodes $v_{11}$ and $v_{22}$ at first layer which is proportional to the similarity of the nodes $v_{11}$ and $v_{22}$ in the input image $J$. The edge weights of the nodes between layers are set to zero to make the object layers disjoint, for example $w\left(v_{11}^{1}, v_{11}^{2}\right)=w\left(v_{11}^{1}, v_{22}^{N}\right)=0$. 
with $w_{i j}^{l}=w\left(v_{i}^{l}, v_{j}^{l}\right)$ obtained from (3.1) and $\mathbf{0}$ is a zero matrix of the size of matrix $L^{l}$ with $|V|$ rows and $N$ columns, where $|V|, N$ are the number of pixels and number of regions in the input image respectively. Then the seeds are manually selected by a user (Section 2.2.1.3) and their boundary conditions will be calculated using (2.11). We finally modify the selected seeds and their corresponding boundary conditions to match to the multi-layer Laplacian matrix.

Assume that at layer $l$ we have a set of all labeled nodes $M^{l}=\left\{m_{1}^{l}, m_{2}^{l}, \ldots, m_{T}^{l}\right\}$ called seeds where $T$ is the number of seeds and the set of all unseeded nodes $U^{l}=\left\{u_{1}^{l}, u_{2}^{l}, \ldots, u_{S}^{l}\right\}$, where $S$ is the number of unseeded nodes in the image graph. Also, assume that $V_{l}$ is the number of all nodes at layer $l$. By partitioning $V^{l}$ into $V_{M}^{l} \mid V_{U}^{l}$, such that $V_{M}^{l} \cup V_{U}^{l}=V^{l}$ and $V_{M}^{l} \cap V_{U}^{l}=\emptyset$, we can rearrange the Laplacian matrix $L^{l}$ to have seeded nodes $L_{M}^{l}$ and unseeded nodes $L_{U}^{l}$ partitioned as

$$
L^{l}=\left[\begin{array}{cc}
L_{M}^{l} & B^{l} \\
B^{l T} & L_{U}^{l}
\end{array}\right]
$$

To create $M^{l}$ and their corresponding boundary conditions $\mathcal{P}_{M}^{l}$ at layer $l$, we modify them as follows

Assume we have a set of initial seeds $M$ and $N=|\mathcal{L}|$ different regions (called labels) with $\mathcal{L}=\left\{\ell_{1}, \ell_{2}, \ldots, \ell_{N}\right\}$. We replicate seeds for each layer to generate $\hat{M}=\left\{\hat{m}_{i_{l}}\right\}$, where $\hat{m}_{i_{l}}$ is the $i^{\text {th }}$ seed at the $l^{\text {th }}$ layer with modified boundary conditions

$$
p_{\hat{m}_{i}^{l}}^{\ell_{k}}= \begin{cases}1 & \text { if } \quad \mathcal{X} \hat{m}_{i}^{l}=\ell_{k}, l=k \\ 0 & \text { otherwise }\end{cases}
$$

where $p_{\hat{m}_{i}^{l}}^{\ell_{k}}$ is the probability of assigning node $\hat{m}_{i}$ to $k^{\text {th }}$ region at layer $l$, and $\mathcal{X} \hat{m}_{i}^{l}$ is the labeled seed $\hat{m}_{i}$ at layer $l$. As a result, the set of unlabeled nodes become $\hat{U}=\left\{\hat{u}_{i}^{l}\right\}$, where $\hat{u}_{i}^{l}$ is the $i^{\text {th }}$ unseeded node at the $l^{\text {th }}$ layer.

Having the new seeds and the modified boundaries, the solution to the segmentation problem will be obtained by replacing $L$ with $\hat{L}, M$ with $\hat{M}, \mathcal{P}_{M}$ with $\mathcal{P}_{\hat{M}}$, and $\mathcal{P}_{U}$ with $\mathcal{P}_{\hat{U}}$ in (2.8). 
The steps of the proposed multi-layer random walker image segmentation method are described in Algorithm 1.

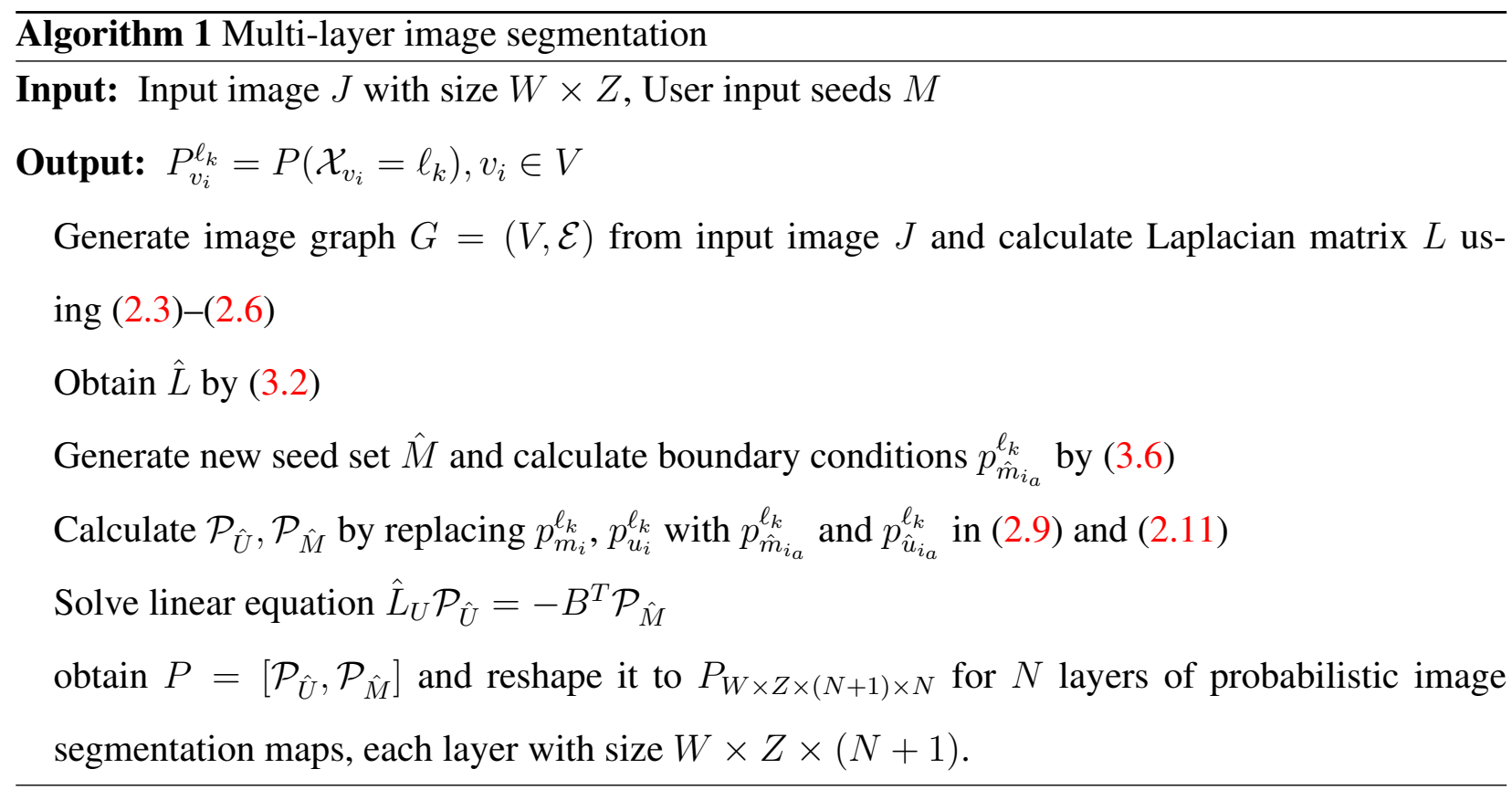

Applying Algorithm 1 will create a set of probabilistic image maps for the $2 D$ image layers $P_{W \times Z \times(N+1) \times N}$; each probabilistic image map belongs to one region in the original image that separates foreground (the desired region) from background in each layer.

However, providing a lot of seeds might sometimes be too time consuming and might make the method perform poorly or not work at all. Therefore, we extend our work to be able to segment multi-layer translucent overlapped objects using soft seed information. 


\subsection{Learning-based Multi-Layer Random Walker Image Seg- mentation}

Supervised image segmentation methods usually start with information extracted from the learning phase to separate an image into non-overlapping regions. We propose a supervised image segmentation technique to segment images with translucent partially-overlapped objects.

Neighborhood patches on image data that represent local geometry on the data can be shown as feature vectors. Although larger neighborhood patches around each pixel give more information for that pixel's texture, it makes the calculations more complex by increasing the dimensions in (2.14). We represented each segment on the image data as a $K$ dimensional feature vector where $K$ is the total number of neighboring pixels.

We examined a few neighborhood systems and chose $K=25$ as a trade-off between complexity and accuracy of the algorithm. Then the probability of assigning image pixels to each segment, called data-term, is obtained by calculating the geodesic distance between the pixels' feature vectors and the segments' feature vectors. This data-term is then used as soft seeds in the RW algorithm instead of user predefined labels.

In multi-label image segmentation by Grady [2], each label has a unique intensity distribution and $\lambda_{i}^{k}$ is simply calculated based on the intensity values using (2.17). This will cause problems with complex structures or large data sizes to give poor results. Some works improved the quality or the computation time of RW image segmentation by improving the feature extraction algorithm [94] or doing offline precomputation [95].

High-dimension feature vectors learn texture of data by representing data on a higher dimensional map. We propose a new feature extractor that finds the similarity between the nodes' and labels' features on a $K$ dimensional feature space.

In the training phase, we use images with ground truth labels and we collect some random 
pixels from each segment. Then for each collected pixel the image intensity values of its $K$ nearest neighbors is gathered and put in a $K$ dimensional feature vector. Each label is then represented as a set of feature vectors for each segment. The geodesic distance for the $K$ dimensional feature vectors is then considered as the similarity measure between feature vectors of the pixels in the image and labels.

\subsubsection{Label Prior Calculation Method}

Assume that we have $M$ images $\mathcal{I}=\left\{I_{m} \mid m=1, \ldots, M\right\}$ in the training phase with $I_{m}\left(v_{i}\right)$ as the intensity value at location $v_{i}$ in image $I_{m}$. Also, assume that we have $N$ partially overlapped objects in each image. These $N$ overlapped objects will create $Y \leq 2^{N}$ non-overlapped regions.

We generate a new label set $\hat{\mathcal{L}}=\left\{\hat{\ell}_{1}, \hat{\ell}_{2}, \ldots, \hat{\ell}_{Y}\right\} \in \mathcal{N}$ where labels $\hat{\ell}_{k}$ represent nonoverlapped segments in the image and $Y=|\hat{\mathcal{L}}|$. We also define $\hat{\mathcal{X}}=\left\{\hat{\mathcal{X}}_{v} \mid \hat{\mathcal{X}}_{v}=\hat{\ell}_{k}, v \in V, k=\right.$ $1,2, \ldots, Y\}$. At each image $m$, we then take $E$ random pixels from each of the $Y$ different regions to make a learning set of size $T=M \times E$ pixels for each region and $S=M \times E \times Y$ total learning pixels.

Assume that we have

$$
I_{m}\left(v_{e}, r\right)=\left\{I_{m}\left(v_{j}\right) \mid v_{j} \in \varepsilon_{v_{e}, r}\right\}
$$

where $\varepsilon_{v_{e}, r}$ is the neighbor pixels of the $e^{t h}$ pixel at image $I_{m}$ (Fig. 3.2), and $r$ defines the distance between pixels

$$
\varepsilon_{v_{e}, r}=\left\{v_{j} \mid \operatorname{Dist}\left(v_{e}, v_{j}\right)<r\right\}
$$

where $\operatorname{Dist}\left(v_{e}, v_{j}\right)$ is the Euclidean distance between pixels $v_{e}$ and $v_{j}$ in the image.

We explore different neighborhood sizes, $r$, and compare computational complexity and accuracy of the algorithm for each $r$. We notices that although increasing the neighborhood size helps improving the accuracy of our algorithm, the rate of improvement decays as the neighborhood size 
increases. Also the complexity of our algorithm exponentially increases with the value of $r$, therefore, we chose $r=2$ as a trade off between complexity and accuracy of the algorithm. The feature vector for the $e^{t h}$ pixel at the $m^{\text {th }}$ image with $r$ neighborhood radius is given as

$$
\Phi_{k}\left(I_{m}, e\right)=\left\{I_{m}\left(v_{e}, r\right) \mid \overline{\mathcal{X}}_{v_{e}}=\bar{\ell}_{k}\right\}
$$

where $\Phi_{k}\left(I_{m}, e\right)$ is a $K$ dimensional feature vector and $I_{m}\left(v_{e}, r\right)$ is obtained using (3.7), (3.8). Then for each region, a learning set is created as

$$
s_{k}=\left\{C_{m, k} \mid m=1,2, \ldots, M\right\},
$$

where

$$
C_{m, k}=\left\{\Phi_{k}\left(I_{m}, e\right) \mid e=1,2, \ldots, E\right\}
$$

Finally, the total learning set becomes

$$
S=\left\{s_{k} \mid k=1,2, \ldots, Y\right\}
$$

Having the learning set $S$ and test image $J$, we then define the similarity measure matrix $D$ of size $|V| \times Y$ as the shortest path between image $J$ pixels' feature vectors and labels' feature vectors where $|V|$ is the total number of pixels in image $J$ and $Y$ is the total number of regions.

$$
D\left(\hat{\mathcal{X}}_{v}=\hat{\ell}_{k}\right)=\min \left\{\cup_{m=1}^{M} \cup_{e=1}^{E} \sqrt{\left(\Phi(J(v))-\Phi_{k}\left(I_{m}, e\right)\right)^{2}}\right\}
$$

where $\Phi_{k}\left(I_{m}, e\right)$ represents the $k$ dimensional feature vector of $e^{t h}$ pixel from $m^{\text {th }}$ image $I_{m}$ for label $\hat{\ell}_{k}$ and $\Phi(J(v))$ represents the $k$ dimensional feature vector for pixel $v$ in image $J$.

Having $D$, we define a new data-term $\lambda_{i}^{k}$ as follows

We first calculate $A_{v}^{k}$ as

$$
A_{v}^{k}=\min \left\{\cup_{j=1}^{Y}\left\{D\left(\hat{\mathcal{X}}_{v}=\hat{\ell}_{j}\right) \mid \mathcal{X}_{v}=\ell_{k}\right\}\right\}
$$




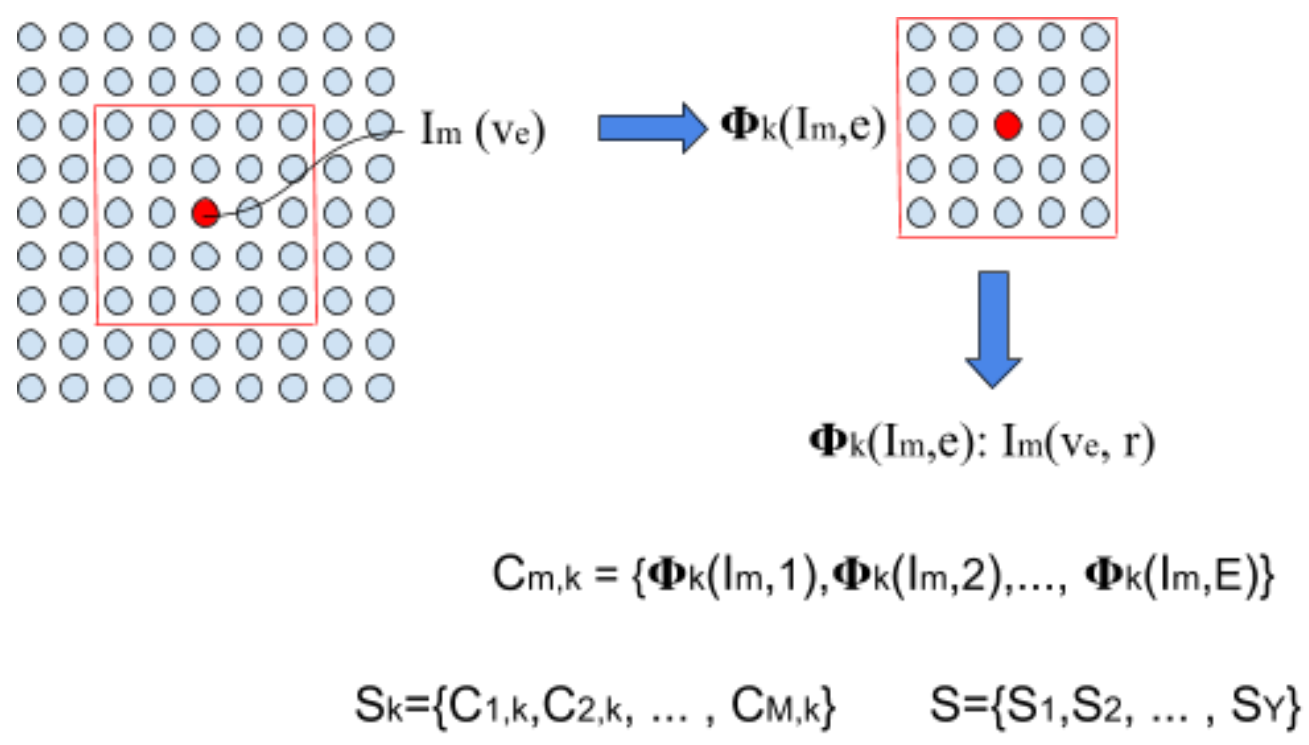

Figure 3.2: Learning set pixels.

where $\ell_{k} \in N$. Then vector $B_{v}$ is $k$ dimensional similarity measure of pixel $v$ to all $N$ labels

$$
B_{v}=\cup_{k=1}^{N}\left\{A_{v}^{k}\right\} .
$$

The new data-term $\lambda_{v}^{k}$ will be calculated as

$$
\lambda_{v}^{k}= \begin{cases}1 & \text { if } \min \left(B_{v}\right)=A_{v}^{k}, \\ 0 & \text { otherwise. }\end{cases}
$$

Combining (2.15) and the multi-layer Laplacian matrix in [3], we have

$$
\left(\hat{L}+\gamma \sum_{r=1}^{N} \Lambda^{r}\right) P^{k}=\lambda^{k}
$$

where $P^{k}, \Lambda^{r}$, and $\lambda^{k}$ are obtained by (2.18), (2.16) and (3.16) respectively, $N$ is the number of partially overlapped regions, and $\hat{L}$ is calculated by (3.2). 

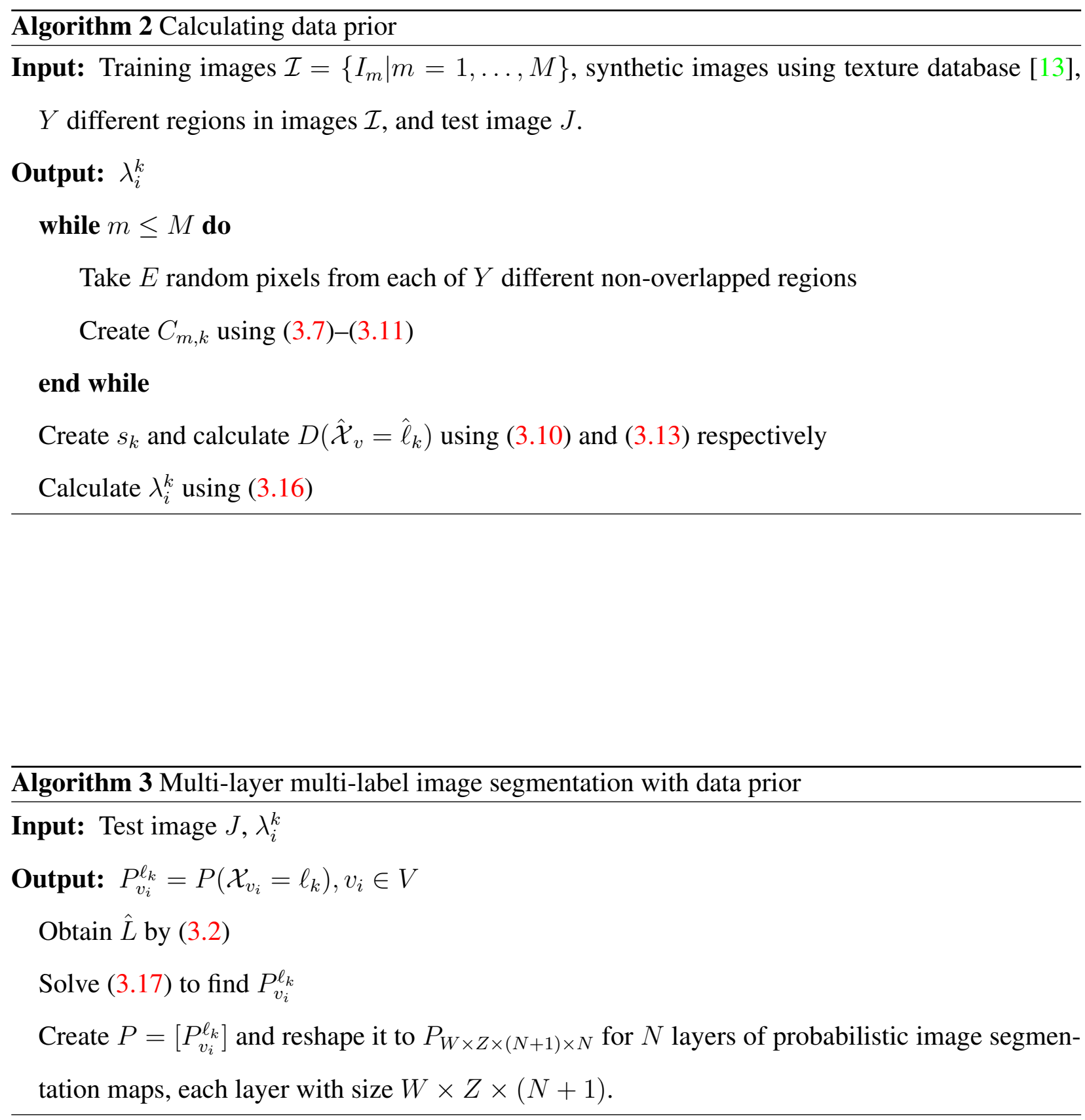
The steps of the proposed multi-layer multi-label random walker image segmentation method using data prior are described in Algorithm 2 and Algorithm 3.

We can also extend the algorithm to add seeds to data prior to improve the results. Assuming there are $\hat{M}$ initial seeds with initial condition values $\mathcal{P}_{\hat{M}}$ and $\hat{U}$ unknown nodes in image $J,(3.17)$ becomes

$$
\left(\hat{L}_{\hat{U}}+\gamma \sum_{r=1}^{N} \Lambda^{r}\right) \mathcal{P}_{\hat{U}}^{k}=\lambda^{k}-B^{T} \mathcal{P}_{\hat{M}}^{k} .
$$

Algorithm 4 describes the steps for multilayer image segmentation with data prior using initial seeds.

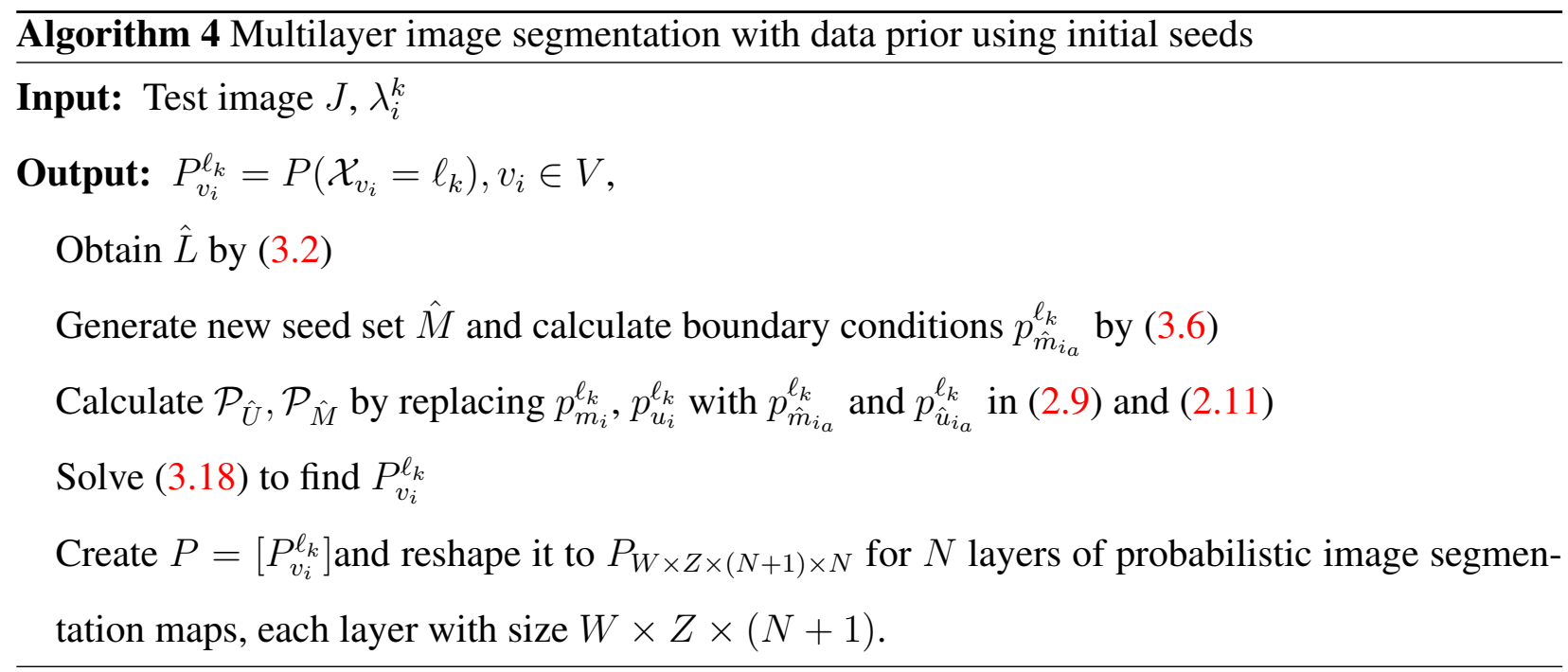

\subsubsection{Data Set-up for Multi-Layer Random Walker Image Segmentation}

To test the multi-layer random walker image segmentation method, we generated synthetic images with a mixture of two or three sources.

We created two or three distorted circles and we added different textures from [13] to these objects to form the image $I_{l}$ as

$$
I_{l}=\sum_{m=1}^{3} a_{m, l} \cdot\left\{\left(O_{m} \cdot P_{m}(x, y)\right) \circledast G\right\},
$$


where $O_{m}$ is the $m_{t h}$ distorted circle using [96] with the size $W \times Z, P_{m}(x, y)$ is the $m_{t h}$ texture from [13] with the size $W \times Z$ from $P_{m}$ with size $F \times H$

$$
P_{m}(x, y)=P_{m}(x: x+W, y: y+Z)
$$

where $x, y$ are random numbers such that

$$
1<x, \quad x+W<F, \quad 1<y, \quad y+Z<H .
$$

". " is dot product, $\circledast G$ is the convolution with the Gaussian filter $G$ of window size of 5 and $\sigma=.5$, and $a_{m}, m=1,2,3$ is a weighting for a linear combination. We added noise to the mixing vector $A=\left\{a_{m} \mid m=1,2,3\right\}$ to create different mixing vectors $A_{l}=\left\{a_{m, l} \mid m=1,2,3, l \in[0,1]\right\}$ for different images

$$
a_{m, l}=a_{m}+N_{l}(0, \sigma), \quad N_{l}=\frac{1}{\sigma \sqrt{2 \pi}} e^{\frac{l^{2}}{2 \sigma^{2}}}
$$

where $l \in[0,1]$ is a random number and $\sigma$ is the power of the noise added to $a_{m}$ and

$$
a_{m}=\{0.4,0.65,0.35\}
$$

Figure 3.3 shows a few samples of the textures and the synthetic image mixtures, respectively.

\subsubsection{Multi-layer User Input Random Walker Image Segmentation Results}

We applied our multi-layer user input RW image segmentation method on these synthetic images. We also applied our multi-layer user input RW method on synthetic images with two partially overlapped cervical cells. Figures 3.4 and 3.5 show the segmentation results for both synthetic datasets.

We calculated the segmentation accuracy by

$$
A c c=\frac{\left|\zeta_{G}=\zeta_{R}\right|}{|V|} \times 100 \%
$$



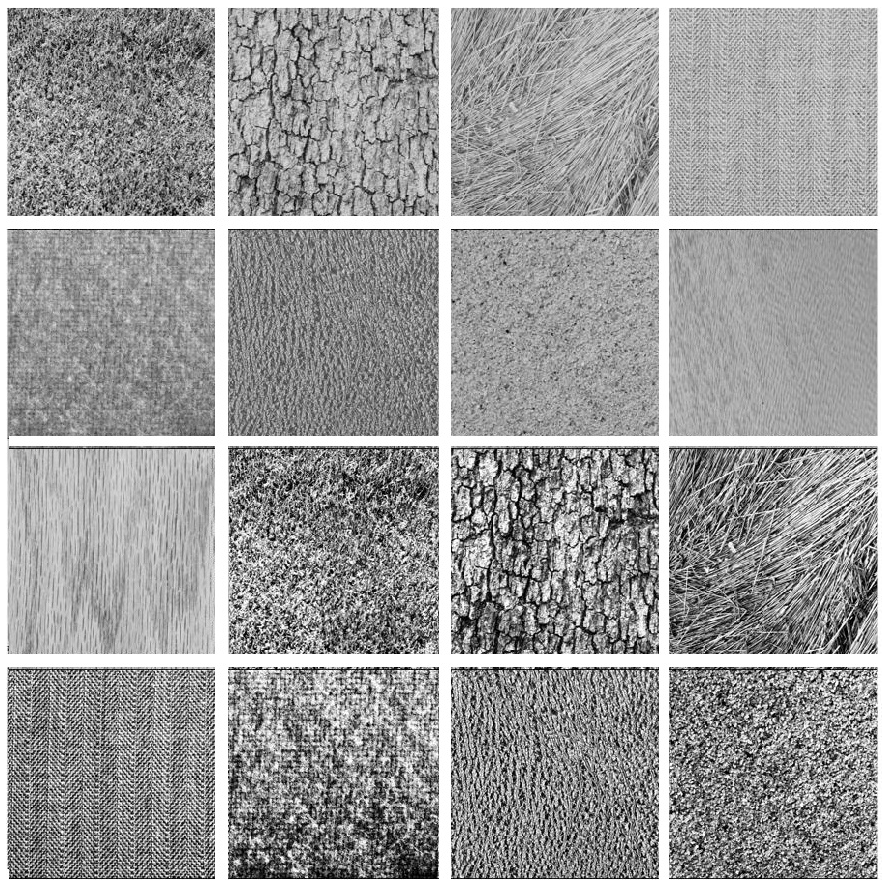

(a)
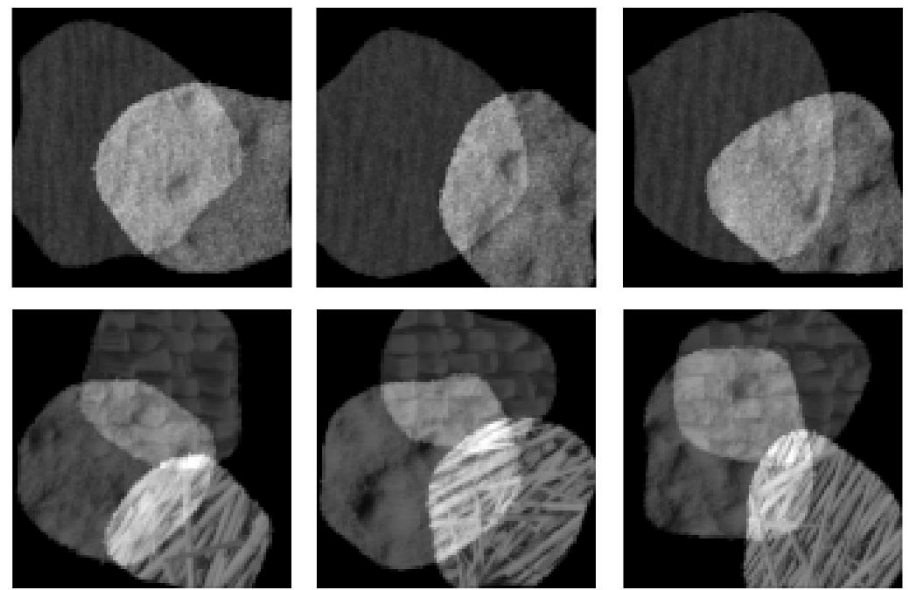

(b)

Figure 3.3: Textures and synthetic images generated to evaluate multi-layer random walker image segmentation algorithm: a) textures [13] and b) image mixtures. 


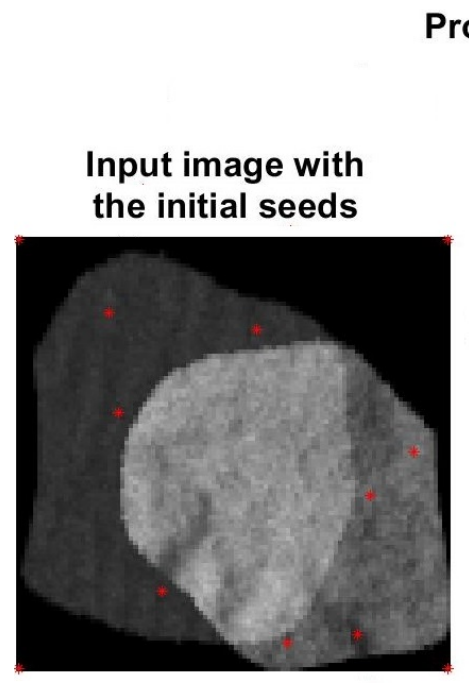

Proposed multi-layer image segmentation

Input image with the initial seeds


Random walker image segmentation
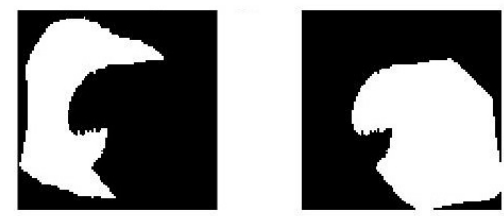

Ground truth image segmentation
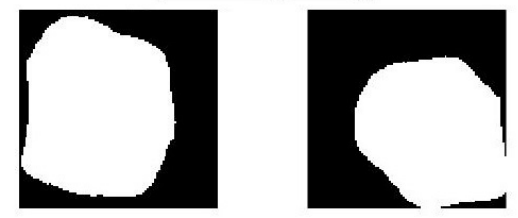

(a)

Proposed multi-layer image segmentation
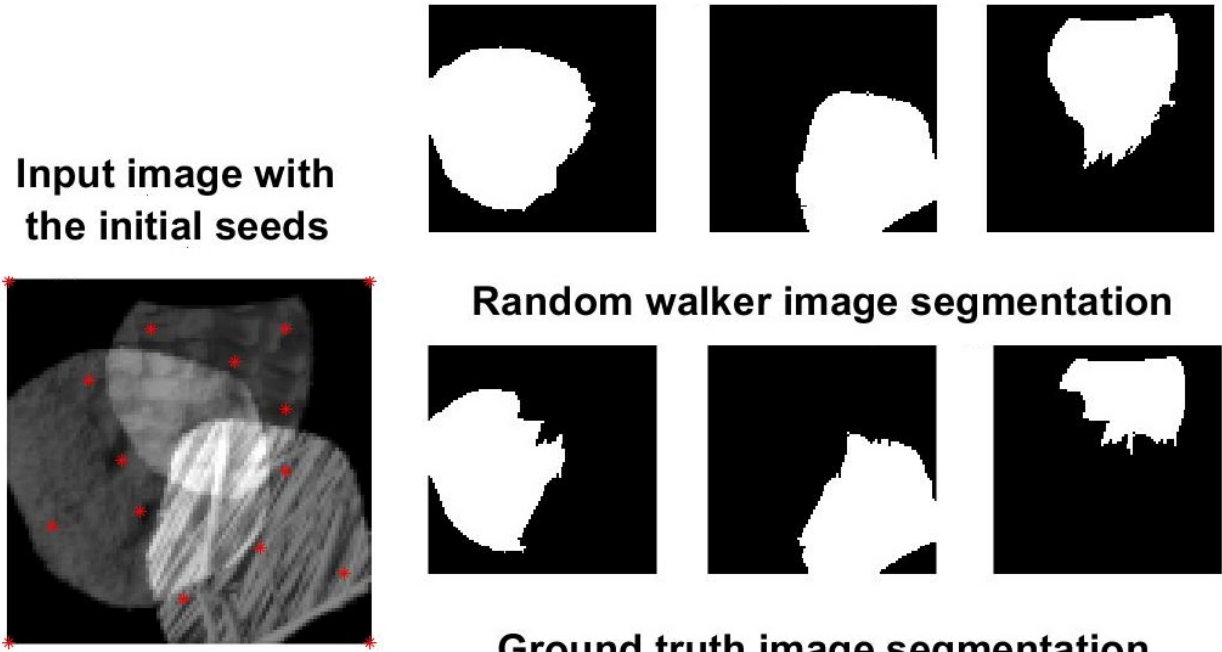

Random walker image segmentation
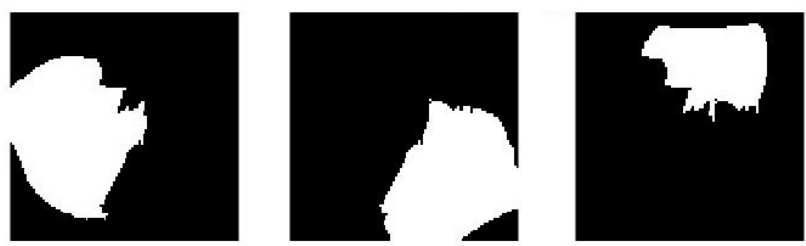

Ground truth image segmentation
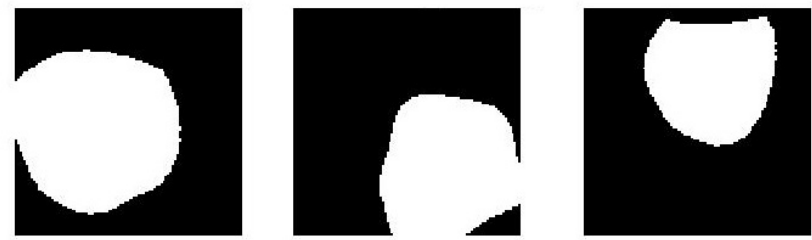

(b)

Figure 3.4: Segmentation results for multi-layer user input random walker image segmentation method (first row), original RW [1] (second row), and the ground truth (third row). a) Image mixture with two sources, b) image mixture of three sources. The seeds are selected by the user. 


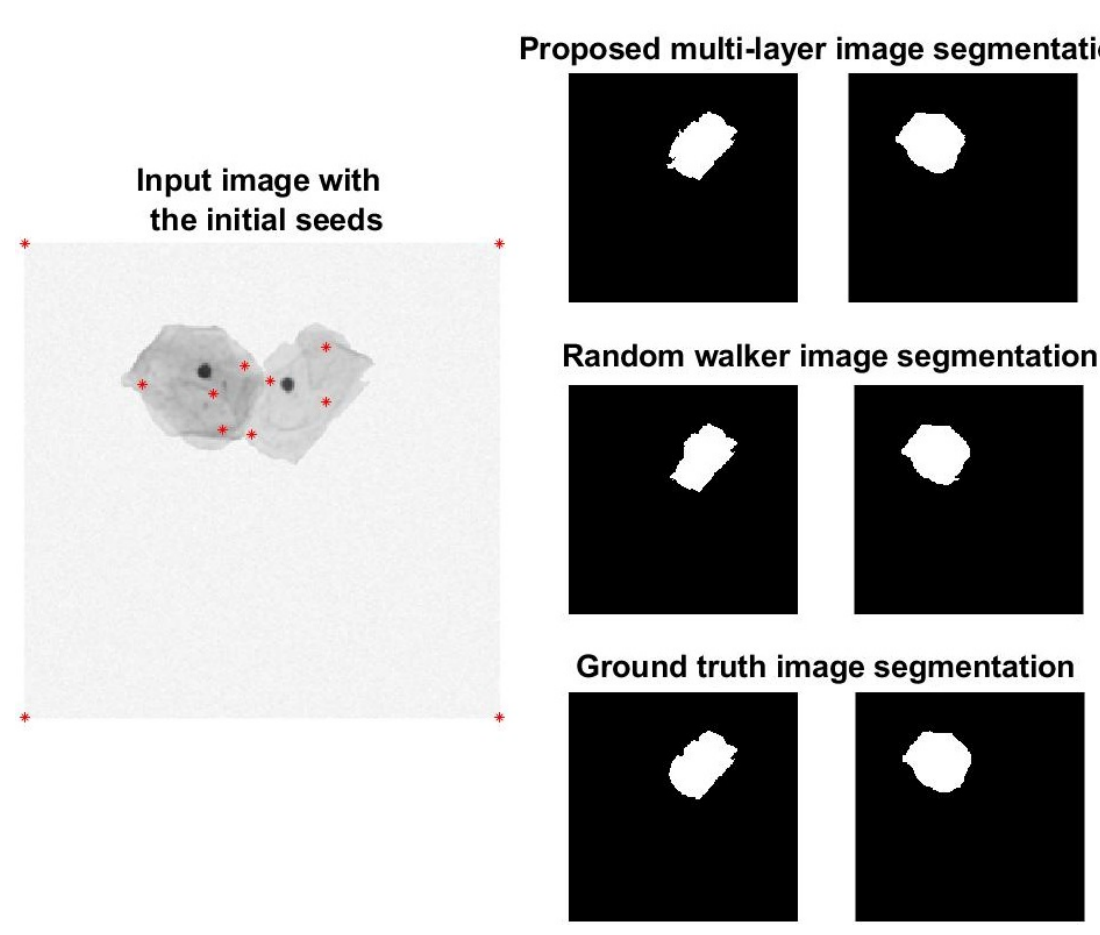

Figure 3.5: Segmentation results for cervical images with input image with initial seeds (left), multi-layer user input random walker image segmentation method (right top), original RW [1] (right middle), and ground truth image segmentation (right bottom). The seeds are selected by the user. 
where $\zeta_{G}=\zeta_{R}$ is a set of nodes where the ground truth labeling $\zeta_{G}$ is the same as the results $\zeta_{R}$ and $|V|$ is the total number of nodes in the image graph. We also measured the intersection of union (IoU) using

$$
I o U=\frac{\zeta_{G} \cap \zeta_{R}}{\zeta_{G} \cup \zeta_{R}} \times 100 \%
$$

where $\cap$ and $\cup$ represent intersection and union of ground truth segmentation and segmentation results, respectively. Table 3.1 shows the accuracy and IOU of the proposed segmentation algorithm compared to the original random walker image segmentation [1] for two and three overlapped sources. The results are averaged over 20 different trials.

Table 3.1: Segmentation accuracy and time complexity for the multi-layer user input random walker image segmentation method and the original random walker image segmentation [1].

\begin{tabular}{|c|c|c|c|c|}
\hline \multirow{2}{*}{ Description } & \multicolumn{2}{|c|}{ Multi-layer RW Method } & \multicolumn{2}{|c|}{ Method in [1] } \\
& Two sources & Three sources & Two sources & Three sources \\
\hline Accuracy(\%) & 98.63 & 97.81 & 91.46 & 92.12 \\
\hline IOU(\%) & 84.1 & 92.8 & 46.3 & 45.6 \\
\hline
\end{tabular}

Table 3.2 shows the accuracy and IOU of our multi-layer user input RW image segmentation algorithm compared to the original random walker image segmentation [1] for synthetic cervical images. The results are averaged over 20 different trials.

\subsubsection{Multi-layer Multi-label Random Walker Image Segmentation Using Prior Results}

We applied our multi-layer multi-label RW image segmentation method on the synthetic distorted circle dataset and synthetic cervical cell dataset. 
Table 3.2: Segmentation accuracy for our proposed multi-layer user input random walker image segmentation method comparing to the original random walker image segmentation [1] on synthetic cervical images.

\begin{tabular}{|c|c|c|}
\hline Description & Multi-layer RW Method & Method in [1] \\
\hline Accuracy(\%) & 99.70 & 99.70 \\
\hline IOU(\%) & 93.52 & 89.86 \\
\hline
\end{tabular}

In the learning phase, we take 50 images and for each image we collect some random pixels from each segment. Then for each collected pixel we put image intensity values of that pixel and its 24 neighbors in a 25-dimensional vector as the feature vector for that selected pixel. Then a probability density function is calculated for each of the segments and is considered as the soft seed values.

Figure 3.6 shows the segmentation results for an input image with three image sources and their ground truth segmentation. In Fig. 3.6, the first column is the original image and the ground truth segmentation. Second and third columns are methods in [1] and [3], respectively. Our proposed data prior segmentation methods with and without seeds are in the last two columns. In Fig. 3.6, segmentation results are provided with one seed for each segment. It can be seen in Fig. 3.6 that when the number of provided seeds is limited (second, third and last columns), segmentation accuracy can be significantly improved by adding a data prior (last column).

Figure 3.7 shows the segmentation results for synthetic cervical cell dataset.

Table 3.3 compares the accuracy of our two proposed segmentation algorithms with the methods in [1], [2], and [3] using (3.24), (3.25). For methods in [1], [3] and our data prior+seeds we provided one seed for each label (last three rows in Table 3.3). The results are averaged over 20 


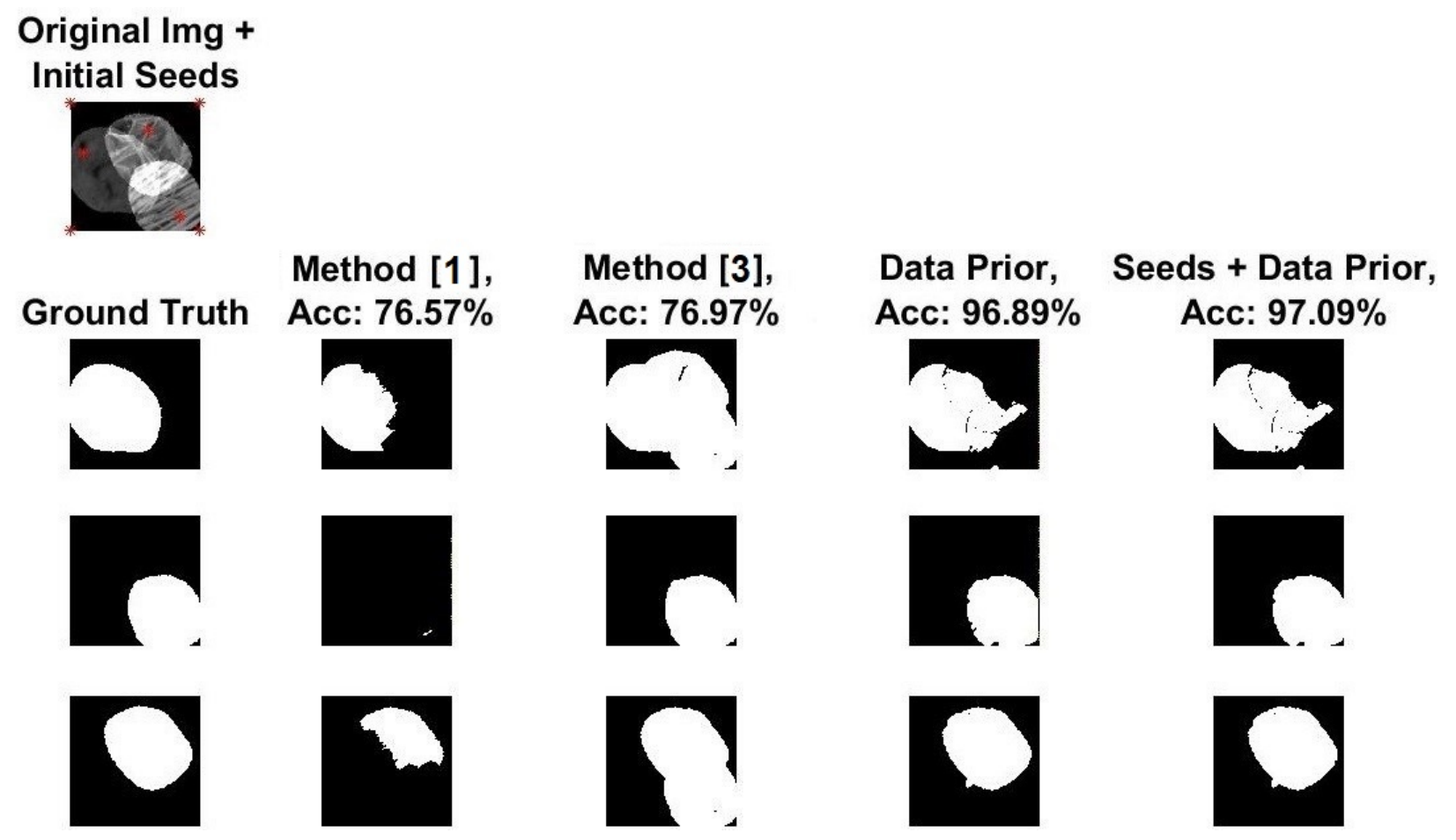

Figure 3.6: The ground truth image with three translucent overlapped sources in the image mixture (first column). Initial seeds that are used for the second, third and last columns are shown as red points in the original image. Segmentation results for [1] and [3] with one seed for each segment (second and third columns, respectively), learning-based multi-layer random walker image segmentation method without seeds (fourth column), and learning-based multi-layer random walker image segmentation method with one seed for each segment (last column). 


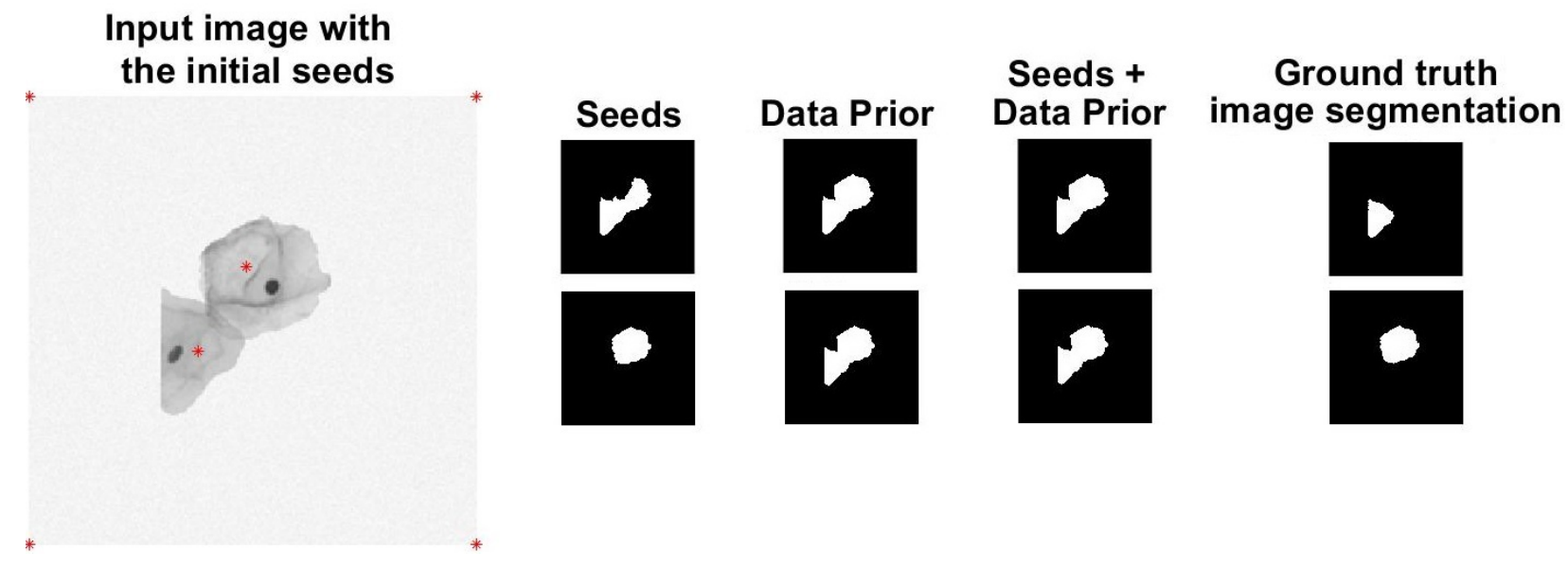

Figure 3.7: Segmentation results for cervical images using multi-layer multi-label random walker image segmentation method. The original synthetic cervical cell (first column), proposed multi-layer user input RW image segmentation method (second column), proposed multilayer multi-label RW image segmentation (third and fourth column), and ground truth image segmentation (last column). The seeds are put by user.

different trials for three translucent overlapped images. All algorithms are run by Matlab software on an $i 7 \mathrm{CPU}$ at $3.6 \mathrm{GHz}$.

In the data prior algorithm by Grady [2], $\lambda_{i}^{k}$ is calculated based on intensity values in the image. Therefore, this method will not work properly for images with more complex textures and will give poor segmentation results. Also, the image segmentation method with seeds by Grady [1] is not designed to deal with the overlapped regions, therefore, its segmentation accuracy is less than the modified version in [3]. Besides, [1] and [3] will not work efficiently when the number of provided seeds is limited (third and forth rows in Table 3.3). Therefore, their results are not good compared to our proposed method. Our proposed methods show significant improvement in segmentation accuracy for complex textures when few or zero number of seeds is provided. 
Table 3.3: Segmentation accuracy and execution time for our proposed multi-layer multi-label RW image segmentation method comparing with the original random walker image segmentation method [1], [2] and our proposed multi-layer user input RW image segmentation method [3].

\begin{tabular}{|c|c|c|}
\hline Segmentation Method & $\operatorname{Accuracy}(\%)$ & $\operatorname{Time}(s)$ \\
\hline Method in [2] & 25.34 & 6.0352 \\
\hline Data Prior (proposed) & 95.55 & 6.0423 \\
\hline Method in [1] & 78.34 & 0.0978 \\
\hline Method in [3] & 86.69 & 0.1332 \\
\hline Data Prior + Seeds (proposed) & 96.02 & 6.0784 \\
\hline
\end{tabular}

Table 3.4 shows the accuracy and IOU of our proposed multi-layer multi-label RW image segmentation algorithm compared to our multi-layer user input RW image segmentation [3] for synthetic cervical images. The results are averaged over 20 different trials.

Although the proposed learning-based RW method performs well for images with different complex textures, it gives poor results for synthetic images with two partially overlapped cervical cells, because of having similar textures. Therefore, we extended our learning method and trained a deep neural network which is explained in the next chapter. 
Table 3.4: Segmentation accuracy and IOU of our proposed multi-layer multi-label RW image segmentation algorithm compared to our multi-layer user input RW image segmentation for synthetic cervical images.

\begin{tabular}{|c|c|c|}
\hline Description & Accuracy $(\%)$ & $\mathrm{IOU}(\%)$ \\
\hline Data Prior & 95.51 & 51.03 \\
\hline Method in [3] & 98.04 & 70.89 \\
\hline Data Prior + Seeds & 95.51 & 50.82 \\
\hline
\end{tabular}

\subsection{Summary}

In this Chapter, we modified original random walker image segmentation algorithm and multi-label random walker image segmentation algorithm to segment images with translucent partially overlapped objects. We created an $N$-layer Laplacian matrix, where $N$ is the number of objects in the input image. We then modified seeds and boundary conditions for each matrix layer. Running the modified algorithm produced multiple image output, each image output belonging to one of the objects in the input image. We then extend our work to select seeds automatically by using a learning method to overcome the time consuming step for user input seed selection. However, finding the proper features is application dependent and is a challenging problem in learning applications. Convolutional neural networks have the advantage of finding important features automatically from the data. Therefore, to improve the seed selection algorithm we used a convolutional neural network. The details of the proposed implemented CNN for image segmentation is explained in the next chapter. 


\section{Chapter 4}

\section{Multi-layer Image Segmentation using CNN}

In the previous chapter we used learning models to improve our multi-layer image segmentation. However, finding proper features for a specific application and data type is a fundamental key in learning systems.

Convolutional neural networks (CNNs) are a subset of deep learning methods recently used widely in computer vision applications, such as image segmentation. One of the biggest advantages of CNNs is that they extract useful features automatically from the data.

To do image segmentation using CNNs, there are two different ways:

- Implement one's own structure and tune the weights using the input/output learning phase. In this approach, the network will be application dependent and the number of layers and weights can be reduced because of the specific dataset that is going to be processed. However, since there are a large number of weights in a $\mathrm{CNN}$ network, this approach needs a lot of time and data for training.

- Start with a pre-trained network and modify the weights with the set of ones own input/output pairs, which is called transferring a network. This approach is usually preferred since one can save on the amount of data needed and the training time.

In this dissertation, we are focusing on using CNNs for image segmentation using images with 
translucent partially overlapped objects. SegNet network [12] is a well-known method for semantic segmentation. However, since the SegNet network is used for a wide range of semantic segmentation applications, we need to use SegNet network [12] as transfer learning. Although SegNet has shown good performance over a wide variety of images, the complexity of the system is high because of a large number of network layers. Also, because it is trained over images with non-overlapping objects, segmentation accuracy might not be excellent for images with translucent partially overlapped objects. Therefore, we implement a new network with fewer network layers and train it from the scratch for our own image segmentation problem.

However, because in the training phase the network starts with random initial weights, the number of training data might need to be increased. Also because of the random start, the convergence of the network might be not promised. Residual networks have shown to improve convergence of the neural network and hence reduce the needed training time. Therefore, the convergence of a new network with random start can be improved by using residual blocks. We implemented a residual CNN with 81 layers. Our proposed CNN is explained in the next section.

\subsection{Proposed CNN}

The SegNet network [12] has 91 layers containing an encoder network, a decoder network and a soft-max pixel-wise classification layer. The encoder network consists of 13 convolutional layers with a kernel size of $3 \times 3$. Each convolutional layer is followed by a batch normalization layer, an element-wise rectified linear unit (ReLU) and a max-pooling layer with $2 \times 2$ window size and stride

of 2. To restore the size of the image, for each max-pooling in the encoder there is an unpooling layer at the decoder network. Each max-unpooling layer is followed by a convolutional layer with kernel size of $3 \times 3$, a batch normalization layer, and a ReLU layer. The network is finally followed by the soft-max pixel classification layer (Figure 2.10). 
For every pixel $z$ in the image, soft-max layer provides a probabilistic vector $\sigma(z)_{i}$ where

$$
\sigma(z)_{i}=\frac{e^{z_{i}}}{\sum_{j=1}^{K} e^{z_{j}}}, \quad i=1, \ldots, K, \quad z=\left(z_{1}, z_{2}, \ldots, z_{K}\right) \in R^{K}
$$

and $\sum_{j=1}^{K} e^{z_{j}}=1$.

Images of size $360 \times 480$ are fed into the network. The output of the network is a $360 \times 480 \times K$

image where each layer represents the probability of each of $K$ classes for each pixel. SegNet then uses a max operator on the soft-max layer to create one output image with non-overlapping segments.

\subsubsection{Proposed CNN for Multi-layer Image Segmentation}

We propose a residual network with 81 layers for multi-layer image segmentation. Similar to the SegNet network, our proposed network consists of an encoder network, a decoder network, a sigmoid pixel classification layer and skip connections between peer blocks at the encoder to the decoder to improve the convergence of the network. Our encoder network has four sets of layers. Each set has 2 or 3 convolutional layers with kernel size of $3 \times 3$ followed by a batch normalization layer and a ReLU layer. At the end of each set we added a max-pooling layer with $2 \times 2$ window size and stride of 2. Similar to the encoder network, our decoder network has four sets of layers. Each set of layers start with a max-unpooling layer followed by a convolutional layer with kernel size $3 \times 3$, a batch normalization layer and a ReLU layer. A threshold operator on sigmoid classification layer at the end provides the multi-layer image segmentation output. Figure 4.1 shows the structure of our proposed network with 81 layers. The list of all layers and their descriptions for the SegNet and the proposed residual network is shown in Appendix A.1, A.2.

Also our designed segmentation $\mathrm{CNN}$ uses an additional set of skip (residual) connections between consecutive blocks at the encoder and the decoder to eliminate singularity in our network and help it to converge when trained [97]. Figure 4.2 shows the overview of the two types of the skip 


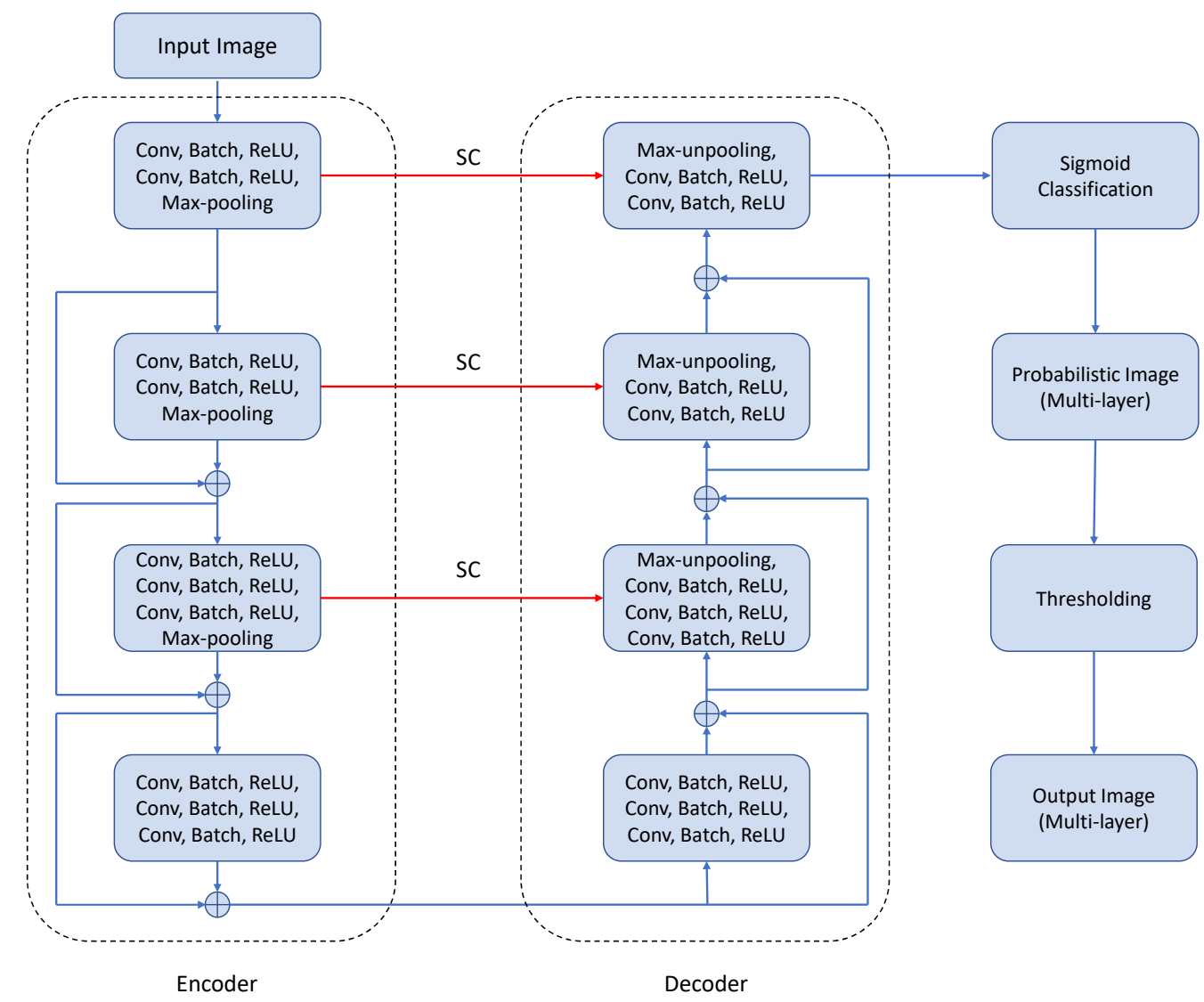

Figure 4.1: Proposed network with residual blocks for image segmentation using translucent partially overlapped objects. 


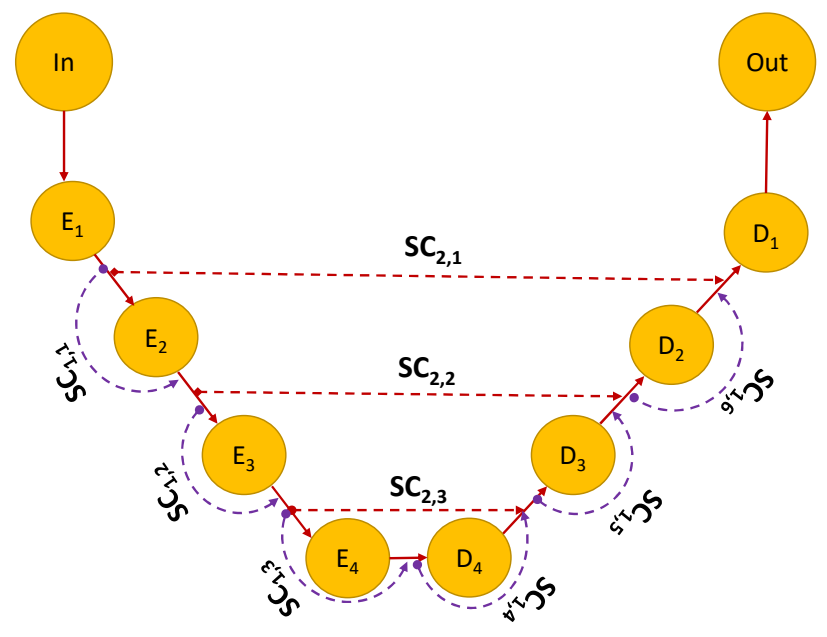

Figure 4.2: An overview of our proposed separation network with $S C_{1, i}, i=1, \ldots, 6$ and $S C_{2, i}, i=1, \ldots, 3$ representing the skip connections within and between encoder and decoder blocks respectively, $E_{i}, D_{i}, i=1, \ldots, 4$ the four encoder and decoder convolutional blocks, and In and Out are the input and output images. Solid arrows represent the normal layer connections while dotted arrows represent the skip connections.

connections in our proposed network. In Figure 4.2, $S C_{1, i}, i=1, \ldots, 6$ and $S C_{2, i}, i=1, \ldots, 3$ represent the skip connections within and between encoder and decoder blocks respectively, $E_{i}$, $D_{i}, i=1, \ldots, 4$ are the four encoder and decoder convolutional blocks, and In and Out are the input and output images. The proposed residual CNN is a modified version of the CNN used in our previous work in [44].

\subsection{Data Set-up for CNN}

We implemented our own proposed residual network with 81 layers with multiple output layers and trained it with our data. 


\subsubsection{Data Set-up for Overlapped Image Segmentation}

In order to evaluate our proposed method, we built three different synthetic datasets.

- A dataset of 700 images including 420 training images and 280 test images was created. Four distorted geometrical shapes with four different textures from [13] were created using

$$
I_{l}=\sum_{m=1}^{4} a_{m, l, t} \cdot\left\{\left(O_{m} \cdot P_{m}(x, y)\right) \circledast G\right\}, t=0,1
$$

where $O_{m}$ is the $m_{t h}$ distorted geometrical shape using [96] with the size $V \times W, P_{m}(x, y)$ is the $m_{t h}$ texture from [13] with the size $W \times Z$ from $P_{m}$ with size $F \times H$

$$
P_{m}(x, y)=P_{m}(x: x+W, y: y+Z)
$$

where $x, y$ are random numbers such that

$$
1<x, \quad x+W<F, \quad 1<y, \quad y+Z<H
$$

". " is dot product, and $\circledast G$ is the convolution with the Gaussian filter $G$ of window size of 5 and $\sigma=.5$. In order to randomly choose two, three or four of the components, we created the mixing vector $A_{l, t}=\left\{a_{m, l, t} \mid m=1,2,3,4, \quad l \in[0,1], \quad t=0,1\right\}$ such that

$$
a_{m, l, t}= \begin{cases}a_{m}+N_{l}(0, \sigma), & \text { if } t=1 \\ 0, & \text { if } t=0\end{cases}
$$

where $N_{l}=\frac{1}{\sigma \sqrt{2 \pi}} e^{\frac{l^{2}}{2 \sigma^{2}}}, l \in[0,1]$ is a random number either 0 or 1 , and $\sigma$ is the power of the noise added to $a_{m}$ and

$$
a_{m}=\{0.4,0.65,0.35,0.85\}
$$

Therefore, we create a set of images mixed of two, three or four objects. For each mixed image $I$ in the dataset, a four-layered image label was created, each layer containing the 


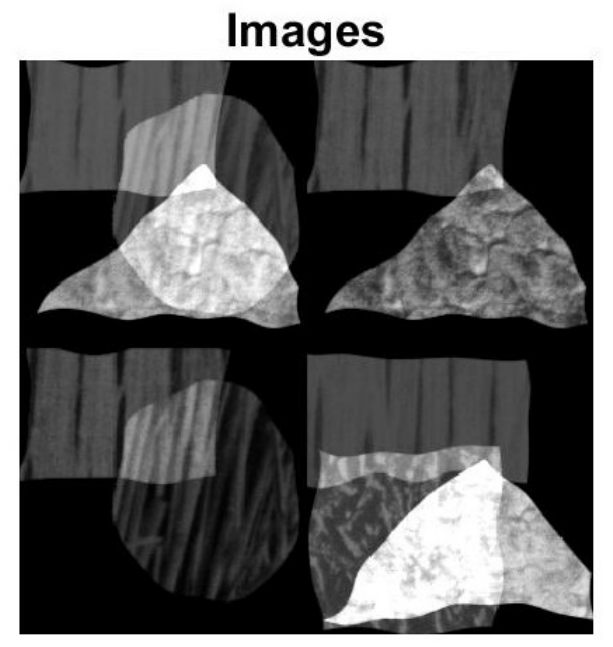

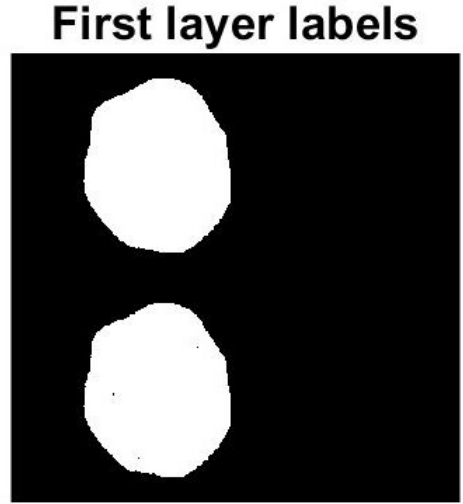

Third layer labels

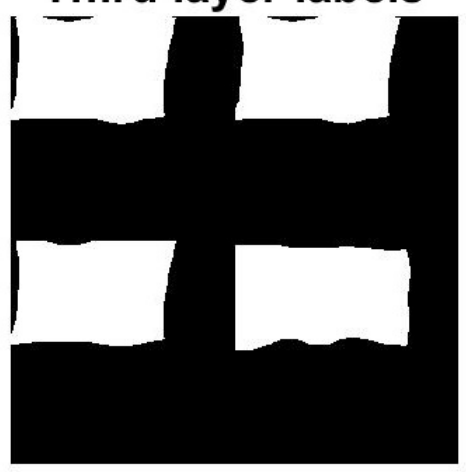

Second layer labels

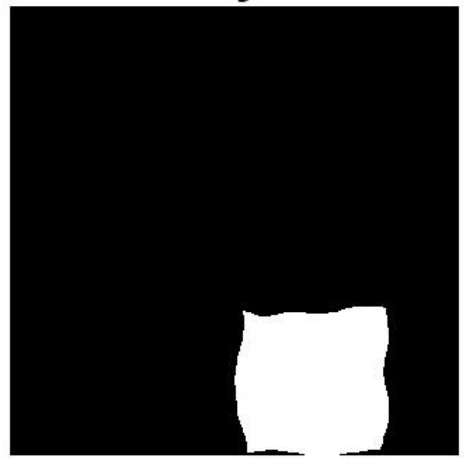

Fourth layer labels

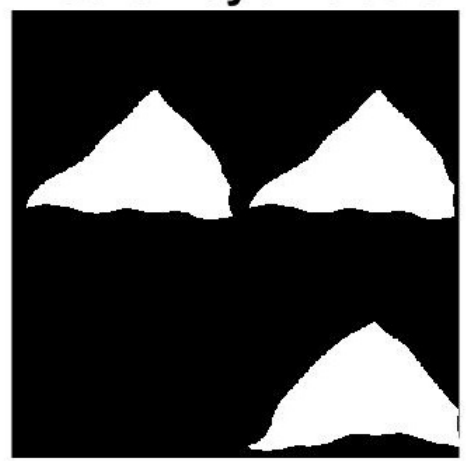

Figure 4.3: A few synthetic images and corresponding labels for the first dataset. The absence of an object is presented with a black object map.

black-and-white map of one of the original shapes. Therefore, the layers are allowed to have overlap, but each layer contains the map of its own object. Figure 4.3 shows a few dataset images and their labels accordingly.

- A dataset of 51000 images including 50000 training images and 1000 test images of size $256 \times 256$ was created. We first picked 100 cells from cytology images and created the ground truth segmentation. Then we applied an affine transform to each cell to generate a variation over the dataset using

$$
F(p)=A p+q
$$


where $p \in O_{m}, A$ is a linear transformation in $\mathbb{R}^{2}, q$ is a displacement vector in $\mathbb{R}^{2}$, and $O_{m}$ is the $m_{t h}$ object and $m=1,2, \ldots, 100$. We then created 2000 images, each having 12 of these transformed cells. Finally, for the 50000 images we picked three images at random, $I_{\text {in }}, n=1,2,3, i=1, \ldots, 50000$, and combined them using alpha blending method (4.7)(4.11) to have a translucent overlapped image.

$$
I_{i}=\sum_{n=1}^{3} R_{i n}+\sum_{n \neq p, n, p=1}^{3} \alpha_{n} J_{i n p}+\sum_{n=1}^{3} \beta_{n} K_{i n}
$$

where $R_{\text {in }}$ is the non-overlapped part of the image layers $I_{\text {in }}$ with $n=1,2,3$ and $i=$ $1,2, \ldots, 50000, J_{i n p}$ represents the partial overlap of all regions $O_{i n}$ and $O_{i p}$ in the $n^{\text {th }}$ and $p^{\text {th }}$ image layers $I_{i n}$ and $I_{i p}$

$$
\begin{array}{r}
J_{i n p}=\left\{Q_{i} \mid Q_{i}=O_{i n} \cap O_{i p}, \quad O_{i n} \in I_{i n},\right. \\
\left.O_{i p} \in I_{i p}, \quad n \neq p, \quad n, p=1,2,3\right\}
\end{array}
$$

and $K_{i n}$ is the partial overlap of all objects $O_{i 1}, O_{i 2}$ and $O_{i 3}$ in three image layers $I_{i 1}, I_{i 2}$ and $I_{i 3}$

$$
K_{i} n=\left\{Q_{i} \mid Q_{i}=\cap_{n=1}^{3} O_{i n} \quad O_{i n} \in I_{i n}\right\}
$$

and

$$
\begin{aligned}
& \alpha_{n}= \begin{cases}0.45 & \text { for } n=1, \\
0.35 & \text { for } n=2, \\
0.45 & \text { for } n=3,\end{cases} \\
& \beta_{n}= \begin{cases}0.2 & \text { for } n=1 \\
0.12 & \text { for } n=2, \\
0.2 & \text { for } n=3\end{cases}
\end{aligned}
$$



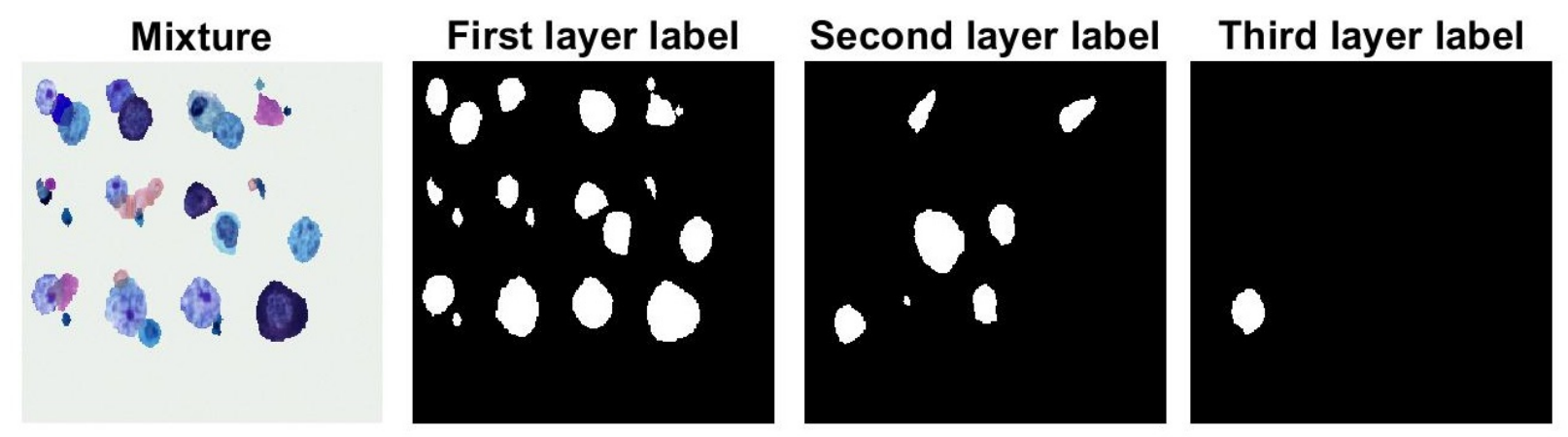

Figure 4.4: A synthetic cytology image and its corresponding labels for the second dataset.

are the coefficients for blending.

The labels consists of layers with segmentation of objects, each layer with non-overlapped objects. Figure 4.4 shows a few dataset images and their corresponding labels.

- We extracted 24 individual cervical cell images from the ISBI 2014 Overlapping Cervical Cytology Image Segmentation Challenge dataset [15]. With these cell images, we applied random affine transforms to increase variation in our cell sample population. Then, we created synthetic images by overlapping and combining two cell images using the Beer-Lambert law [14]. The detailed information about how to create this dataset is explained in Chapter 5. Following the above approach, we generated 51000 synthetic images including 50000 training images and 1000 test images by overlapping and combining real cervical cell images using Beer Lambert Law [14]. Fig. 4.5 shows a few synthetic cervical cell images.

\subsubsection{Results}

The effect of adding vertical skip connections are already discussed in many networks such as SegNet [12]. In order to show the effect of adding vertical skip (residual) connections to our network in terms of number of needed training data, training time and overall segmentation accuracy, we 



Figure 4.5: A few synthetic image mixtures of partially overlapped two cervical cells using BeerLambert law [14].

Table 4.1: Segmentation accuracy.

\begin{tabular}{|c|c|c|c|c|}
\hline Description & Accuracy $(\%)$ & IoU $(\%)$ & Training Time $($ Sec $)$ & Training Data \\
\hline Net1 & 99.37 & 97.59 & 3043 & 420 \\
\hline Net2 & 99.55 & 98.07 & 829 & 420 \\
\hline
\end{tabular}

compared two networks with and without vertical skip connections (Table 4.1). In Table 4.1, Net1 and Net2 represent CNNs without and with vertical skip connections, and accuracy and IOU are calculated by (3.24), (3.25) for 280 test images. We used our first synthetic dataset to evaluate $N e t 1$ and Net2.

It is seen in Table 4.1 that with the same amount of training data, the training time for Net2 is significantly improved along with the slightly improve on the accuracy and IOU for test images.

We trained our proposed network for our first dataset on Matlab platform with 50 epochs respectively. For the second and third datasets, we trained our proposed network on a Python platform with 50 epochs using 50000 training data of size $256 \times 256$. We used a learning rate of 0.001 and 
explored a few optimizers, such as stochastic gradient descent (SGD) [98], stochastic gradient descent with momentum (SGDM) [16], Rmsprop [99], Adam and AdaMax [100]. We then chose the Adam optimizer because it converges faster for our datasets. Figures 4.6, 4.7, 4.8 show the results of the segmentation for all three datasets.

Figure 4.6 shows the segmentation results for the first datasets using our proposed network. The first row represents the output segmentation, the second row represents the ground truth segmentation and the third row shows the input image and the four original sources. As can be seen in Fig. 4.6 the input image has three sources and the last source is set to zero. The last image in the second row is set to blank as there are only three sources while the other three images are the ground truth segmentation of the corresponding sources in the input image.

Figure 4.7 represents the segmentation results for the second dataset. The left image represents the original image mixture with overlapped regions. The right image in the figure is the ground truth segmentation of the image mixture. Three image segmentation layers are shown as red, green and blue colors in the image respectively. Starting from the first segmentation layer, the objects that are overlapped with first layer objects are moved to the second segmentation layer and the overlapped objects with the first and second layers are moved to the third segmentation layer. The middle image in the figure represents the segmentation results. Same as ground truth image segmentation, red, green, and blue colors in the image represent objects in first, second, and third image segmentation layers, respectively.

In Figure 4.8, left represents the original synthetic cervical cell composed of two real individual cells, top and bottom right represent the ground truth and segmentation results respectively. Table 4.2 shows the accuracy of the segmentation results calculated by (3.24), (3.25) for our three datasets. For the first dataset the results are averaged over 280 test images. For the second and third datasets the results are averaged over 1000 test images.

In Table 4.2, we applied our proposed residual network on our three datasets where First, 


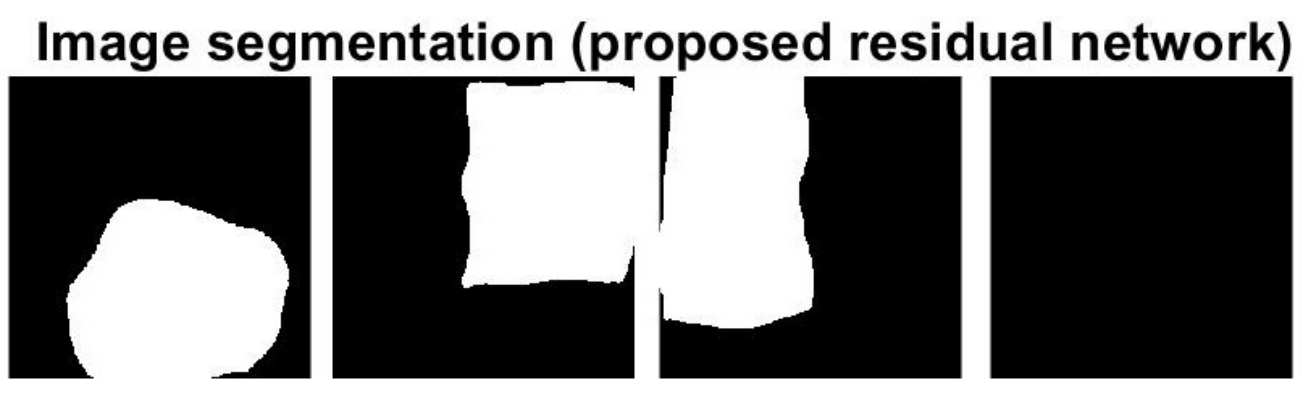

\section{Ground truth image segmentation}
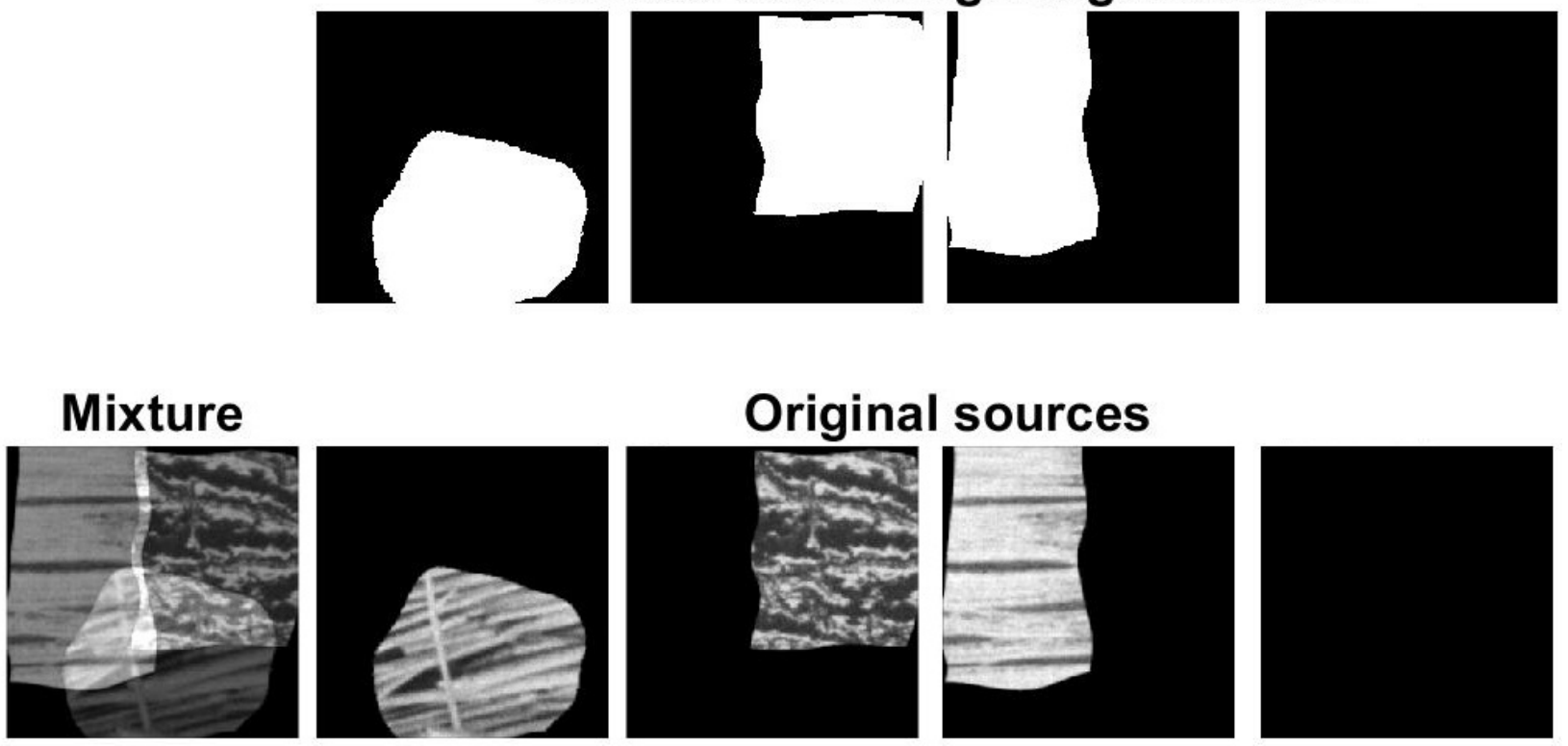

Figure 4.6: Results for the proposed network on first dataset. Top: segmentation results, middle: ground truth segmentation, bottom: original image mixture and image sources. 


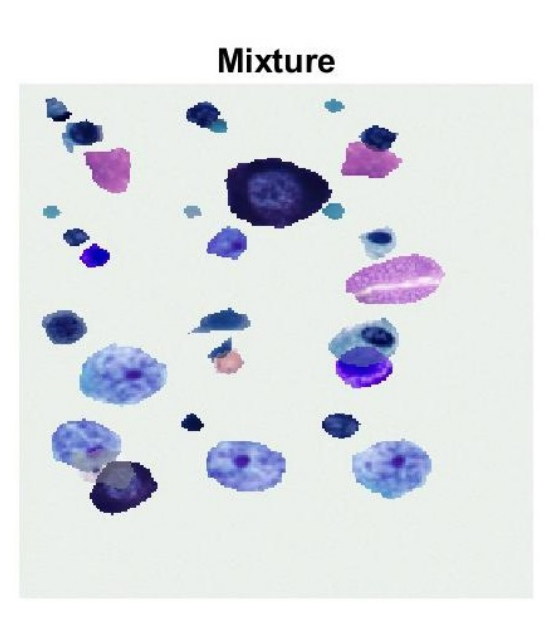

\section{Image segmentation} (proposed residual network)
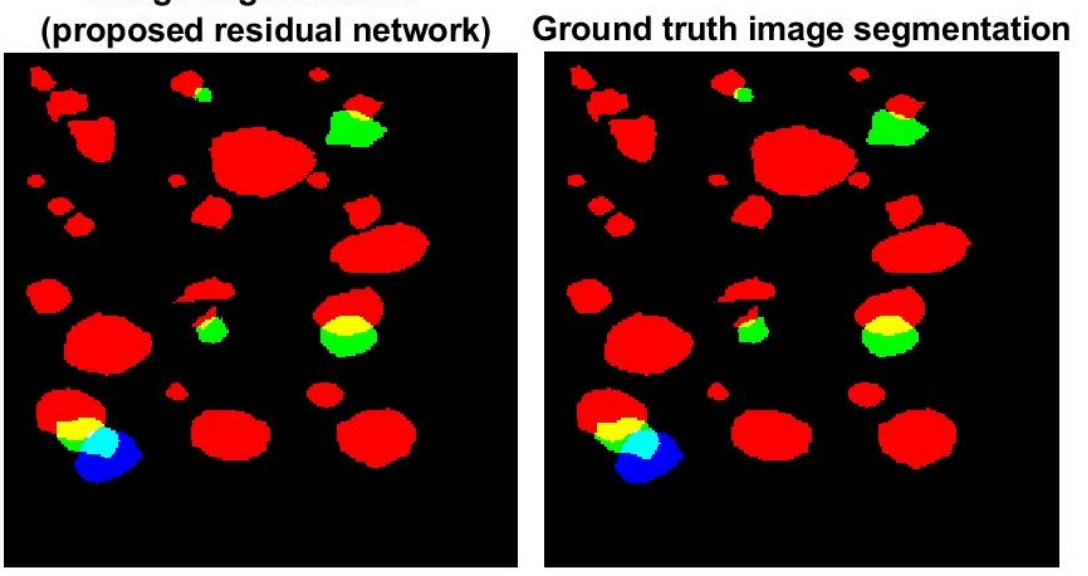

Figure 4.7: Results for the proposed network on our second dataset. Left: original image mixture, middle: segmentation results, right: ground truth.

Second and Third represent the three datasets. Comparing to our first dataset, for the second and third datasets we created much more complex images in order to be close to real cytology image datasets. Therefore, we increased the number of training images for both second and third datasets. We then used this segmentation results on our third dataset for image decomposition. Our proposed two stage CNN for image decomposition is explained in Chapter 6.

\subsection{Summary}

In this chapter, we implemented a convolutional neural network to segment images with translucent partially overlapped objects. Taking the advantage of shape of SegNet network and residual networks we designed a network with U-shape and residual blocks to improve the convergence of network and reducing the number of training data. Although the proposed network performs well for simple datasets, the current implementations of CNNs are not good to find the precise edge borders for cervical cell segmentation. Therefore, we used the proposed CNN to find an approximate 
Mixture

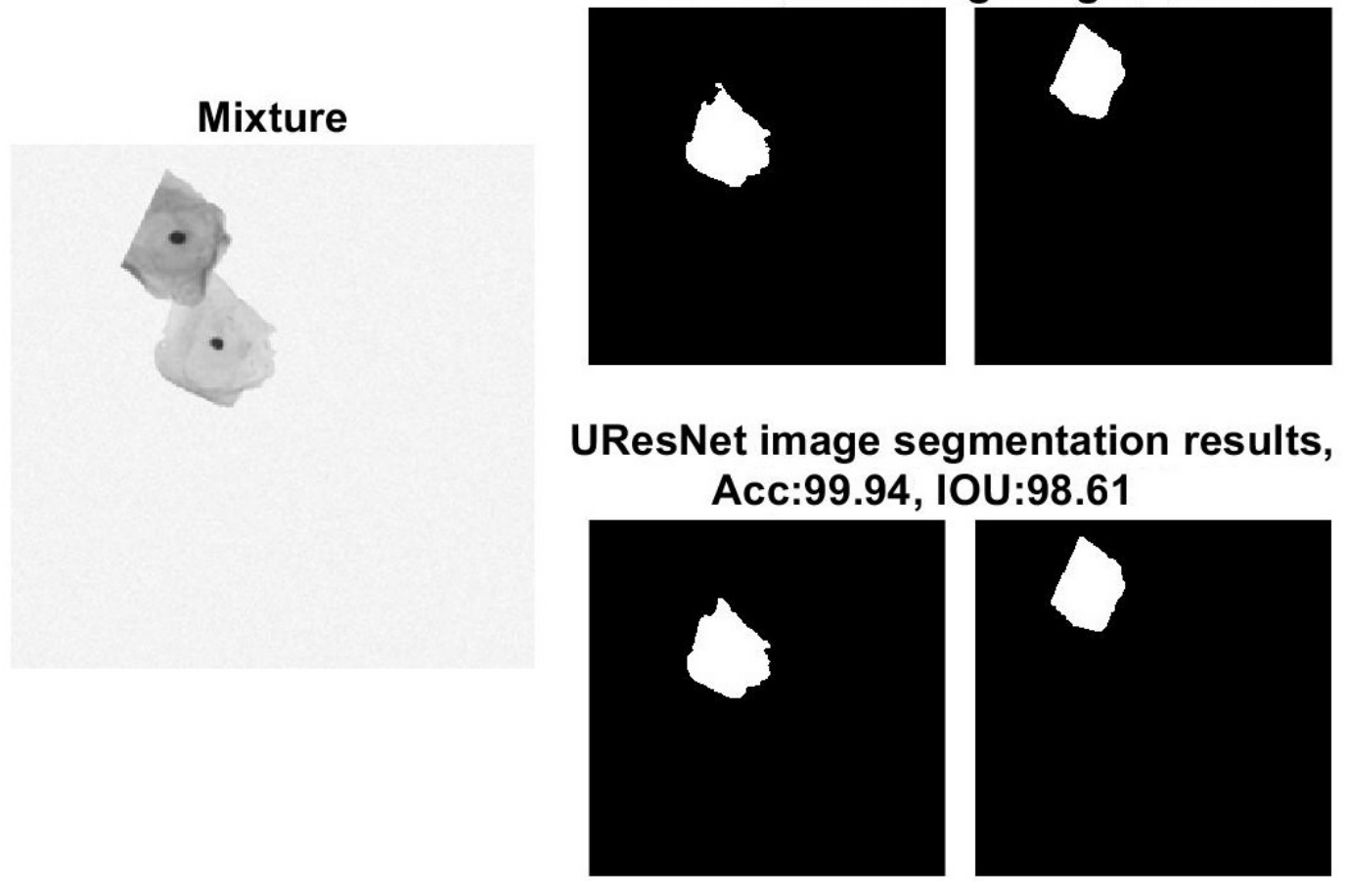

Ground truth image segmentation

UResNet image segmentation results,

Figure 4.8: Results for the proposed network on third dataset. Left: original image mixture, top right: ground truth segmentation, bottom right: segmentation results.

cell segmentation and then we used a fine tuning method to refine the cell borders (Chapter 5). We finally used the segmentation masks obtained from our proposed CNN-based method in Chapter 5 for image separation in Chapter 6. 
Table 4.2: Segmentation accuracy.

\begin{tabular}{|c|c|c|c|}
\hline Description & Accuracy $(\%)$ & $\operatorname{IoU}(\%)$ & Time $(\mathrm{Sec})$ \\
\hline First & 99.55 & 98.06 & 829 \\
\hline Second & 99.78 & 95.81 & 101320 \\
\hline Third & 99.87 & 97.17 & 100870 \\
\hline
\end{tabular}




\section{Chapter 5}

\section{Multi-layer Random Walker Image Segmentation for Overlapped Cervical Cells using Probabilistic Deep Learning Methods}

In $[4,43]$, the authors created a multi-step approach for overlapping cervical cell segmentation using a CNN. They refine the results of the CNN for accurate segmentation. Although a CNN is good at generalizing the training, it is not very good at getting highly precise edges in current implementations for cervical cell segmentation $[4,43]$. Neural networks' inability to perform highly precise edges stem from their intrinsic practical limitations on computational complexity and training data. CNNs for image processing utilize multiple layers of convolutional filters followed by max-pooling layers which reduce the image resolution. Therefore, a CNN is good to give a rough segmentation of the cells, and then we have to do edge refinement afterwards.

The random walker image segmentation method is good at region growing so it can be used as the refinement step to fine tune the CNN segmentation results. However, random walker struggles when it has poor initial setup, so if the seeds are not extracted correctly, the final segmentation results will be poor.

By using a combination of $\mathrm{CNN}$ and random walker image segmentation, we take advantage of 


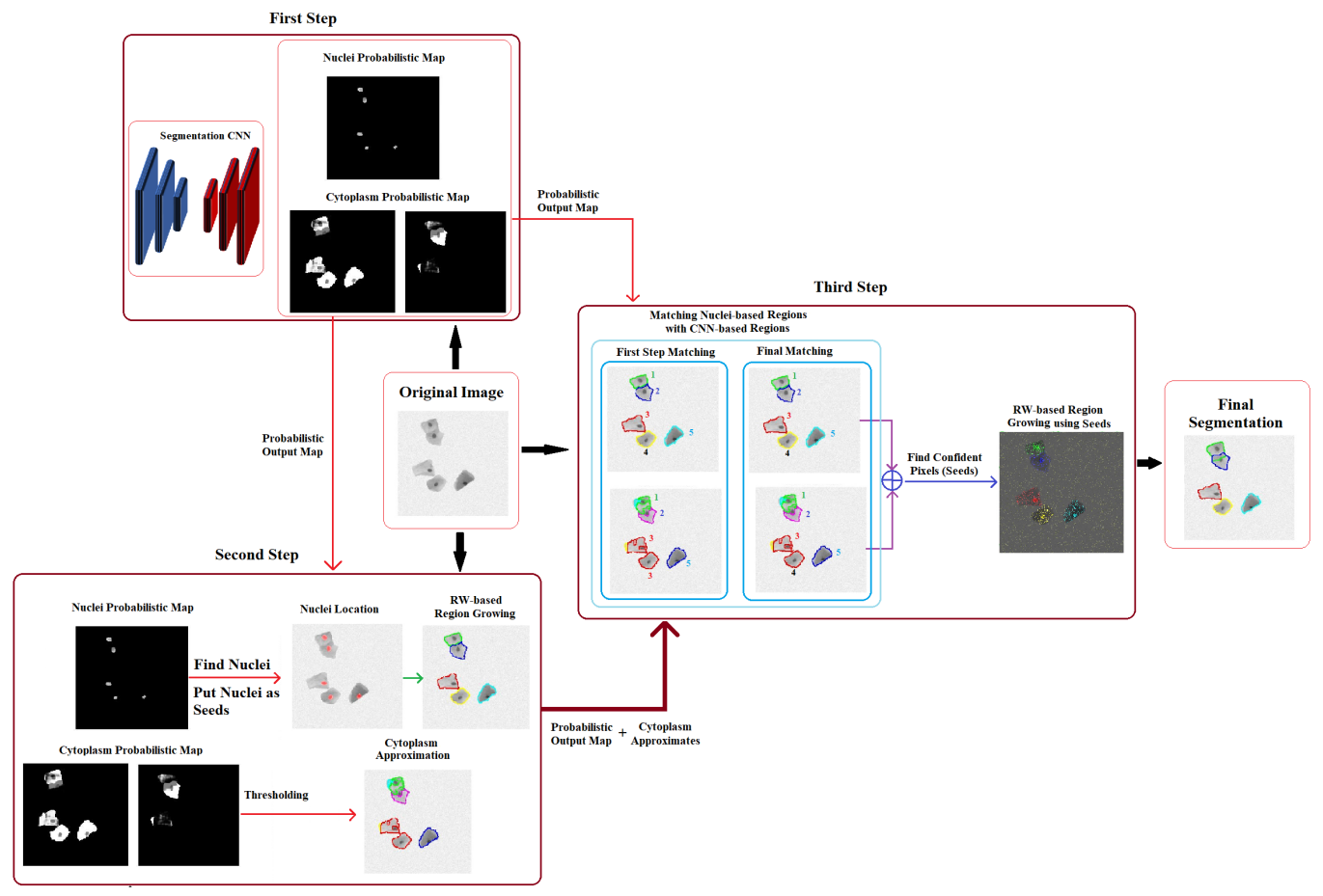

Figure 5.1: Proposed three-step image segmentation method for jointly overlapped cells dataset. The confident pixels in third step are pixels with high probability values from CNN probabilistic output maps.

each part and remove their disadvantage. Different from other methods that use discrete segmentation results from $\mathrm{CNN}[4,43]$ for fine tuning, we use a CNN probabilistic map to try to guide the random walker at the refinement step.

\subsection{Proposed CNN-based Image Segmentation for Overlapped Cervical Cells}

We propose two three-step methods for partially overlapped cervical cell segmentation. Our threestep proposed image segmentation methods are shown in Figures 5.1 and 5.2. 


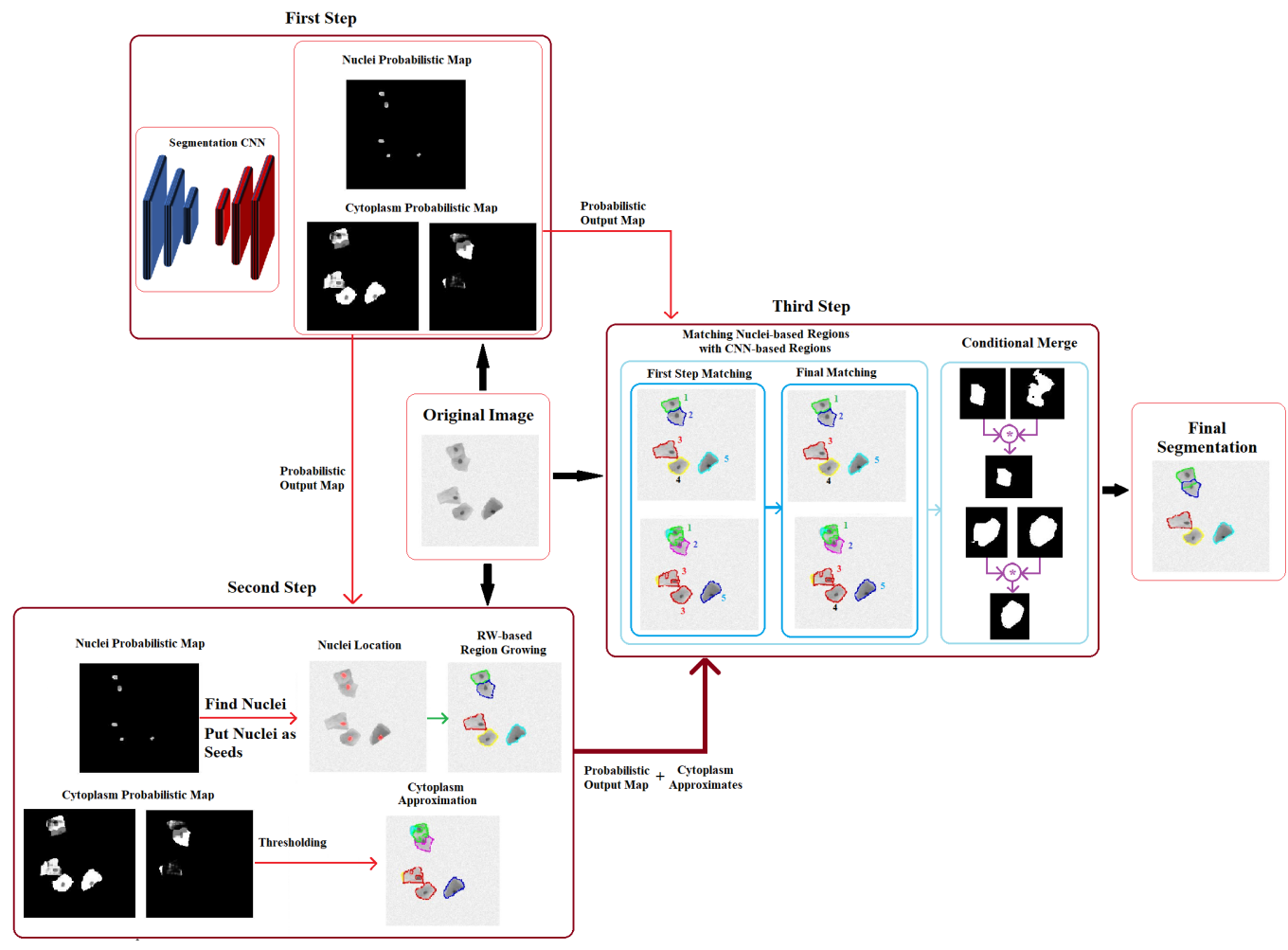

Figure 5.2: Proposed three-step image segmentation method for multiple overlapped cells dataset.

In Figure 5.1, the final step of the proposed method is shown for one cell for simplicity. In Figures 5.1 and 5.2, matching in the third step tries to match the cytoplasm segmentation obtained from the nuclei-based cytoplasm approximation and CNN output cytoplasm approximation.

In the first step in Fig. 5.1, 5.2 we use a proposed residual CNN to generate probabilistic maps for cell nuclei and cytoplasms. In the second step, we chose nodes that have high probability in the CNN nuclei probabilistic output map and use these nodes as seeds for a multi-layer random walker image segmentation for nuclei-seeded region growing. In this same step, we also find cell cytoplasm candidates by thresholding the CNN cytoplasm probabilistic output maps to obtain a cytoplasm approximation across the image. In the third step, we first find a one-to-one correspondence between the results of nuclei-seeded region growing and cytoplasm approximation. To make 
the correspondence, we use the Hungarian algorithm [49]. We then modify the corresponding regions by checking the similarity between them. If the similarity of the matching regions is less than a threshold we select confident pixels from nuclei-based region growing segments and use them as seeds to modify the borders of the cytoplasms in the cytoplasm approximation segments. We then apply two different methods called jointly overlapped and multiple overlapped cervical cell segmentation.

For the first proposed method, we merge the information of the corresponding regions to find a set of new seeds for the final multi-layer random walker image segmentation algorithm. For the second proposed method we apply conditional merging on the corresponding segments to obtain the final segmentation. The following subsections provide details of the two proposed approaches.

\subsubsection{Proposed Residual CNN}

The residual $\mathrm{CNN}$ is designed with 81 layers consisting of an input layer (1 layer), an encoder network (36 layers), a decoder network (36 layers), a logistic sigmoid layer (1 layer), a probabilistic output layer (1 layer), and three sets of skip connection layers, each having a convolution layer and a normalization layer (6 layers). The structure of the residual CNN is shown in Fig. 5.3. Residual neural networks have been shown to improve convergence, to reduce training time, and to allow for deeper networks with improved accuracy [34]. Hence, the designed CNN in Fig. 5.3 uses residual connections to allow a deeper network that will still converge when trained. The proposed residual $\mathrm{CNN}$ is a modified version of the CNN used in our previous work in [45] and is augmented to produce the probabilistic image maps.

The encoder network is broken down into four groups of layers. Each group has 2 or 3 convolutional layers with kernel size of $3 \times 3$ followed by a batch normalization layer and a ReLU layer. At the end of each group, a max-pooling layer is included with $2 \times 2$ window size and stride

of 2. Residual connections are made across consecutive groups of layers with an addition layer 


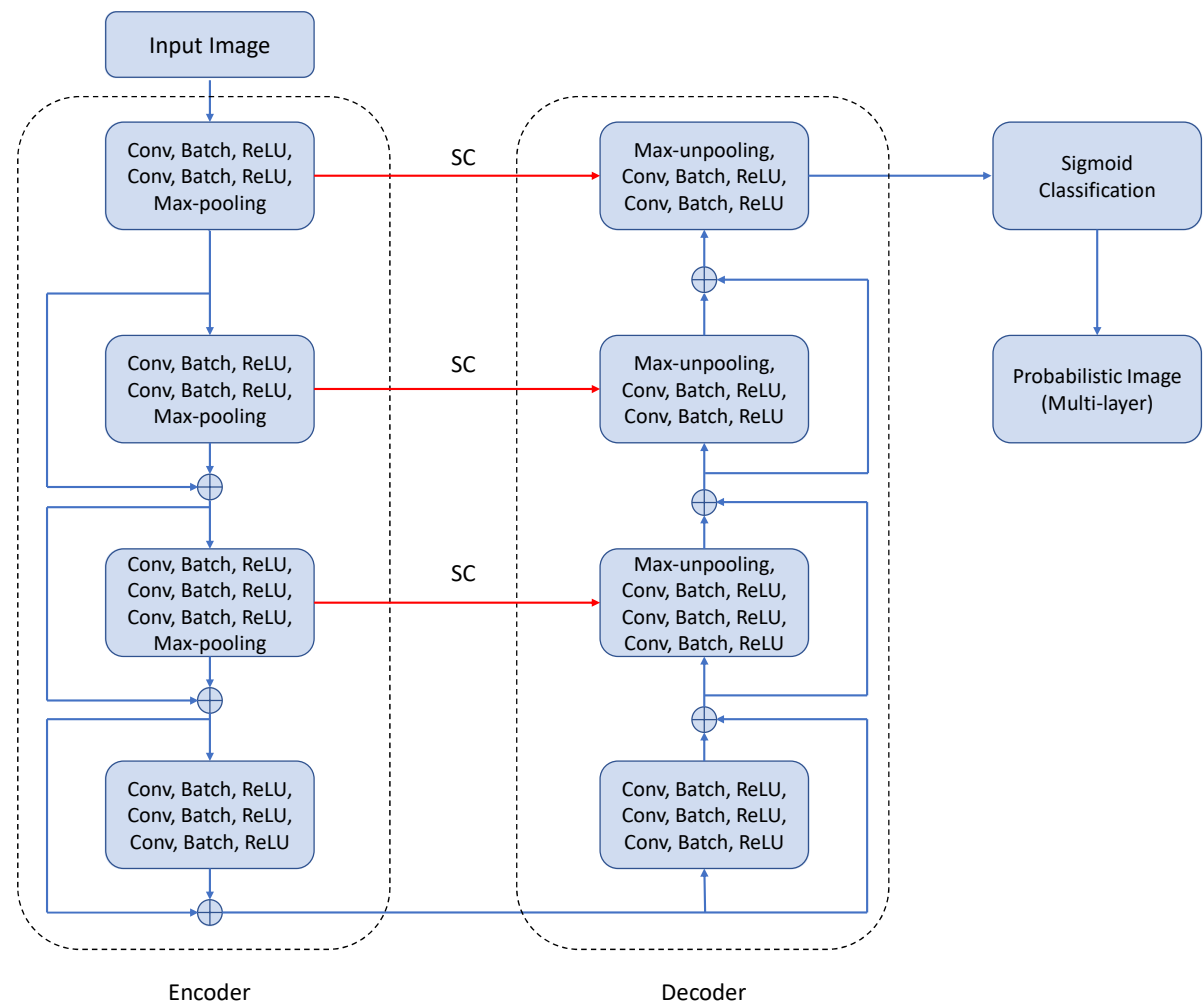

Figure 5.3: Proposed residual $\mathrm{CNN}$ for initial probabilistic image segmentation that produces multi-layer probabilistic image maps (first step of Fig. 5.1, 5.2).

included between groups. Similar to the encoder network, the decoder network has four groups of layers. Each group of layers starts with a max-unpooling layer followed by a convolutional layer with kernel size $3 \times 3$, a batch normalization layer and a ReLU layer. Also, residual connections are made across consecutive groups of layers with an addition layer between groups.

In the proposed residual $\mathrm{CNN}$, we also include skip connections between each group of encoding layers to their corresponding group of decoding layers following the approach for U-Net [101]. A sigmoid layer at the end of the CNN provides the output probabilistic image maps. Details of our network with 81 layers is explained in Appendix (A.2). 

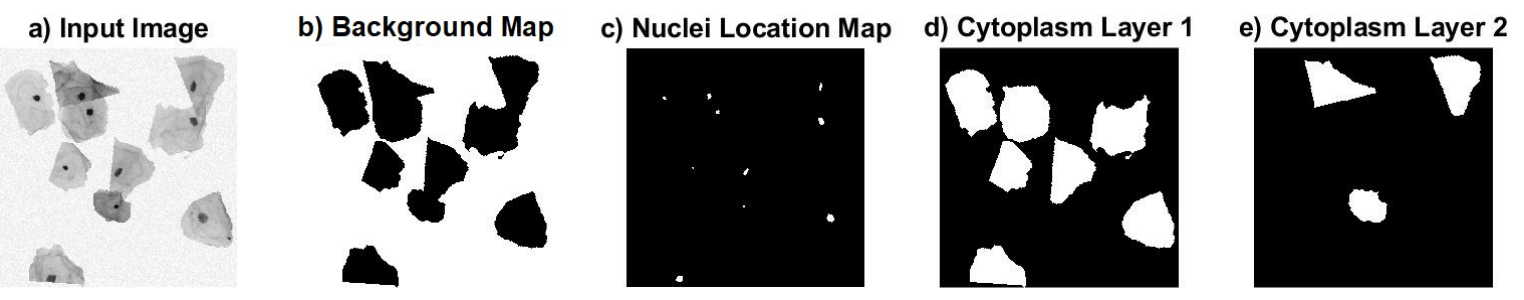

Figure 5.4: A training sample containing (a) the $\mathrm{CNN}$ input image and the binary training masks for the (b) background, (c) nuclei, and (d)-(e) two non-overlapping cytoplasm images.

Since training a CNN works best with a large amount of data, we created images from individual real cervical cell images that we synthetically manipulated, overlapped, and combined. By synthetically creating the overlapped cell images from individual real cervical cells, we can also generate binary ground truth images for the background, cell nuclei, and all of the individual cell cytoplasm for training of the CNN. To train our proposed CNN, we generate two datasets called jointly overlapped cervical cell dataset and multiple overlapped cervical cell dataset.

For the jointly overlapped cervical cell dataset, 50000 images with ground truth labels are provided as the training samples for the $\mathrm{CNN}$, each training sample containing the input image with the binary images of background layer, nuclei layer, and two non-overlapping cytoplasm layers. Figure 5.4 shows an example of the training data.

For multiple overlapped cervical cell dataset, 100000 images with ground truth labels are provided as the training samples for the $\mathrm{CNN}$, each training sample containing the input image with the binary images of background layer, nuclei layer, and four non-overlapping cytoplasm layers. Figure 5.5 shows an example of the training data with ground truth labels. 
a) Input Image

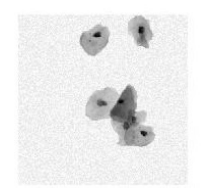

b) Background

Layer

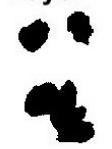

c) Nuclei Layer

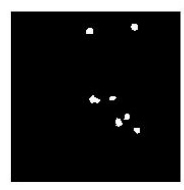

d) Cytoplasm

Layer 1

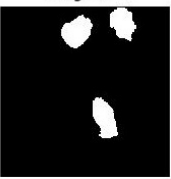

e) Cytoplasm

Layer 2

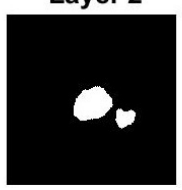

f) Cytoplasm

Layer 3

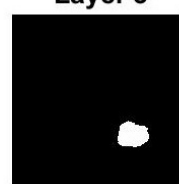

g) Cytoplasm

Layer 4

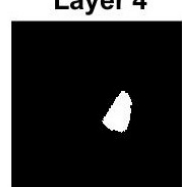

Figure 5.5: A training sample containing (a) the $\mathrm{CNN}$ input image and the binary training masks for the (b) background, (c) nuclei, and (d)-(g) four non-overlapping cytoplasm images.

\subsection{Jointly Overlapped Cervical Cell Segmentation}

A dataset of 50000 images with ground truth labels are provided as the training samples for the $\mathrm{CNN}$. Each training sample containing the input image, an image of up to $N$ partially overlapping cells and the binary images for the background $I_{b}$ where there are no cells, the locations of cell nuclei $I_{n}$, and the cell cytoplasm $I_{c_{1}}$ and $I_{c_{2}}$ without overlap at each layer. Note that $I_{c_{1}}$ and $I_{c_{2}}$ may each contain multiple cells, but organized where no overlap exists in each layer.

\subsubsection{Data Set-up}

We extracted 65 individual cervical cell images from the ISBI 2014 Overlapping Cervical Cytology Image Segmentation Challenge dataset [15]. Figure 5.6 shows a few samples from the dataset.

With these cell images, we applied random affine transforms to increase variation in our cell sample population. Then, we created synthetic images by overlapping and combining cell images using the Beer-Lambert law [14].

he Beer-Lambert law describes the attenuation of light traveling through materials [14]. Given a background light intensity $E_{B}$, object molar attenuation coefficient $\epsilon$, the material's attenuating species molar concentration $\gamma$, the object's light traveling distance $L$, and the object's transmitted 


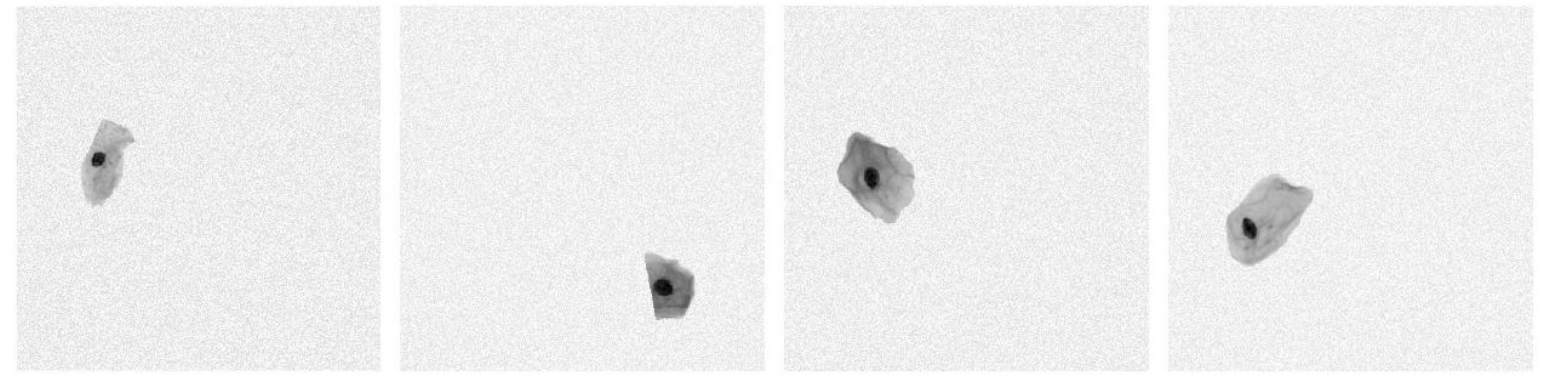

Figure 5.6: A few samples of individual cervical cells from the ISBI 2014 Overlapping Cervical Cytology Image Segmentation Challenge dataset [15] to create jointly overlapped cervical cell dataset using the Beer-Lambert law [14].

light $E_{T}$, the Beer-Lambert law gives the optical intensity profile as [14]

$$
E_{T}=E_{B} e^{-\epsilon \gamma L}
$$

Consider that we have a set of cells $C=\left\{c_{1}, \ldots, c_{N}\right\}$ where in any particular region $x$ only $N_{o}(x) \leq N$ cells overlap. If each cell $c_{i}$ has transmitted light $E_{c_{i}}, i=1, \ldots, N$, then in a particular region $x$ with only $N_{o}(x)$ cells, the transmitted light through the overlapped cells $E_{T}(x)$ can be calculated as

$$
E_{T}(x)=E_{B}^{1-\sum_{i \in C_{o}(x)} \alpha_{i}(x)} \prod_{i \in C_{o}(x)} E_{c_{i}}^{\alpha_{i}(x)}
$$

where $C_{o}(x) \subseteq C$ is the subset of overlapping cells at $x$ and $\alpha_{i}(x)$ is a control parameter for the thickness of a cell in the overlapping region $x$ [14]. Figure 5.7 shows an example of applying the Beer-Lambert law when $N_{o}(x)=2$ where the two cells overlap. For the cell overlap in Fig. 5.7, $E_{T}(x)=E_{B}^{1-\alpha_{c_{1}}(x)-\alpha_{c_{2}}(x)} E_{c_{1}}^{\alpha_{c_{1}(x)}} E_{c_{2}}^{\alpha_{c_{2}(x)}}$.

Following this approach, we generated 50000 synthetic images by overlapping and combining real cervical cell images using (5.2). Images were produced with 10 cervical cells and any particular region $x$ has a maximum overlap of two cells (i.e., $\forall x, N_{o}(x) \leq 2$ ). To determine $E_{B}$ in (5.2), we averaged over the background area for all the training images in the ISBI 2014 dataset [15]. 


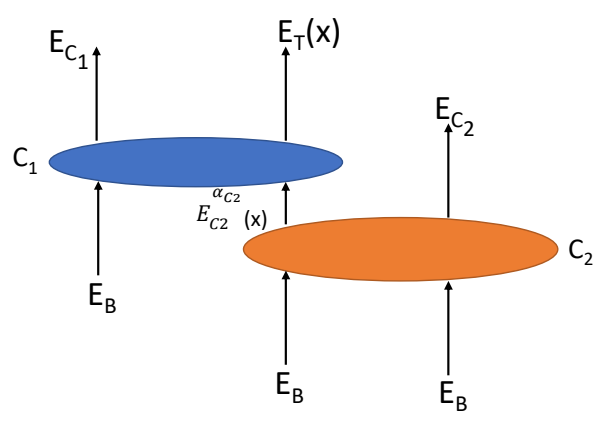

Figure 5.7: Illustration of the Beer-Lambert law for two overlapping cells [14].
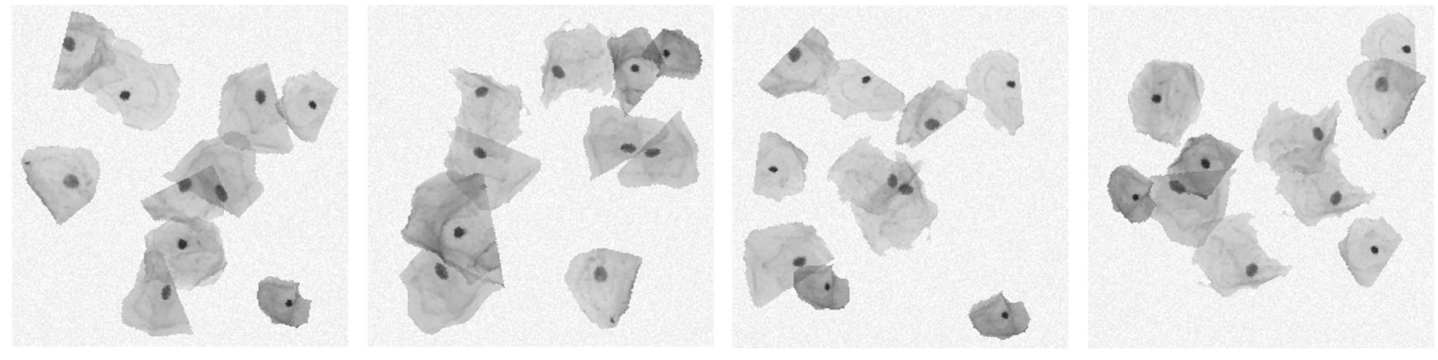

Figure 5.8: A few synthetic image mixtures from jointly overlapped cervical cell dataset, created using Beer-Lambert law using (5.2) for $N_{o}(x) \leq 2$.

Also, the ordered set $[0.95,0.9]$ was used for $\alpha_{i}(x)$ for $N_{o}(x) \leq 2$ as the coefficients to control the difference of the thickness in the overlapping area. We rotated and translated the cells from the ISBI 2014 dataset [15] and the percentage of overlap for cells in each image was randomly selected. Figure 5.8 shows a few synthetic image mixtures created using this approach.

Algorithm 5 explains generating our dataset.

\subsubsection{Nucleus and Cytoplasm Mapping}

An input image $I$ is given to the proposed residual network in the first phase. At the second phase of the proposed method, we take the probabilistic image maps from the residual CNN for 
Algorithm 5 Generating dataset synthetically manipulated from real individual cervical cells for jointly overlapped cervical cell segmentation.

Input: 65 Real individual cervical cells with size $512 \times 512$ from ISBI 2014 dataset [15].

Output: Synthetically generated dataset $D$ with 50000 images, each image containing image mixture, segmentation map of cells and original individual cells.

Repeat for 50000 times

1) Randomly choose two cells from the real individual cervical cells and put them in two separate image layers.

2) For each image layer

$1-$ Repeat four times

2 - Choose one cell from the real individual cervical cells and apply random rotation to the cell.

3- Apply a random translation to the cell to remove its probable overlap with other cells in the same layer.

3) End.

4) Combine the two image layers using (5.2) with random percentage of overlap for cells in each image and put it to the dataset $D$.

5) Put the two-layer image segmentation map and two-layer image separation of individual cells along to its corresponding image mixture.

End.

Down sample the images in $D$ to the size $256 \times 256$. 
the background $I_{b}$, cell nuclei $I_{n}$, and the two cell cytoplasm layers $I_{c_{1}}$ and $I_{c_{2}}$, and use these to generate an $N$-layer probabilistic image map, where each layer contains the map of individual segmented cells and $N$ is the number of nuclei in $I_{n}$. The steps taken for this second phase are outlined in the following paragraphs.

\subsubsection{Nuclei and Approximate Cytoplasm Detection}

We first find the location of the nucleus for each cell and the approximate location of the cells' cytoplasm by using a threshold $t_{i}$ on the $\mathrm{CNN}$ output probabilistic maps using

$$
B_{i}= \begin{cases}1, & \text { if } I_{i}>t_{i}, i \in\left\{b, n, c_{1}, c_{2}\right\} \\ 0, & \text { otherwise }\end{cases}
$$

where $B_{i}$ is a binary image containing the approximate location of the background, nuclei or cytoplasm, and $t_{i}$ is a threshold value for layer $i$. We then apply the morphological connected component algorithm [102] on $B n$ to create an $N$ layer nuclei masks $M_{n, l}, l=1,2, \ldots, N$, where $N$ is the number of detected nuclei in $B n$. Having the binary masks $M_{n, l}, l=1,2, \ldots, \ldots, N$, $B_{b}$ we then select the seeds and apply a multi-layer random walker image segmentation algorithm (Algorithm 1) to extract the approximate cytoplasm candidates. The details are explained in the following paragraphs.

Nuclei-based Seed Selection From the $N$-layer nuclei masks $M_{n, l}$, we set the nuclei probabilistic image maps $P_{n, l}$ as

$$
P_{n, l}(x)= \begin{cases}I_{n}(x), & \text { if } M_{n, l}(x)=1 \\ 0, & \text { otherwise }\end{cases}
$$

where $P_{n, l}(x)$ is the probability of the $l^{\text {th }}$ cell's nuclei at pixel $x$ in layer $l$. 
We also create the background probabilistic map

$$
P_{B}(x)= \begin{cases}I_{b}(x), & \text { if } B_{b}(x)=1 \\ 0, & \text { otherwise. }\end{cases}
$$

Once the probabilistic image maps for nuclei locations $P_{n, l}$ and background $P_{b}$ are obtained, we define a set of seed pixels that we are confident belong to different image regions. We define the foreground confident pixel set as

$$
C_{l}=\left\{x \mid P_{n, l}(x)>T_{f}\right\}, \quad l=1,2, \ldots, N
$$

where $x$ represents the pixels in the image and $T_{f}$ is a threshold value for foreground seed selection. We also define the background confident pixel set as

$$
C_{b}=\left\{x \mid P_{b}(x)>T_{b}\right\}
$$

where $P_{b}(x)$ is the probabilistic image map of background and $T_{b}$ is the threshold value for back-

ground seed selection. In other words, for each layer $l$ or $b$, we collect the pixel $x$ as foreground seed if $P_{n, l}(x)>T_{f}$ or as background seed if $P_{b}(x)>T_{b}$. For the setup, we use empiricallyselected threshold values of $T_{f}=0.2$ and $T_{b}=0.9$. We use these pixels as seeds for the proposed multi-layer random walker image segmentation algorithm.

Figure 5.9 shows an example of approximating cells' cytoplasm with original image in (a), the probabilistic image map of the $\mathrm{CNN}$ in (b)-(e), seed selection in (f), confident pixels as seeds for cytoplasm (red) and background (green) in (g), nulcei-based cytoplasm approximation output and its corresponding probabilistic map in (h)-(i).

After selecting the seeds, we apply Algorithm 6 to extract the $N$-layer approximate cytoplasm candidates.

Proposed nuclei-based Multi-layer Random Walker Image Segmentation Assume we have an input image $I: \mathcal{R}^{2} \mapsto \mathcal{R}$ of size $m \times n$ with a mixture of $N$ partially overlapped objects. The 


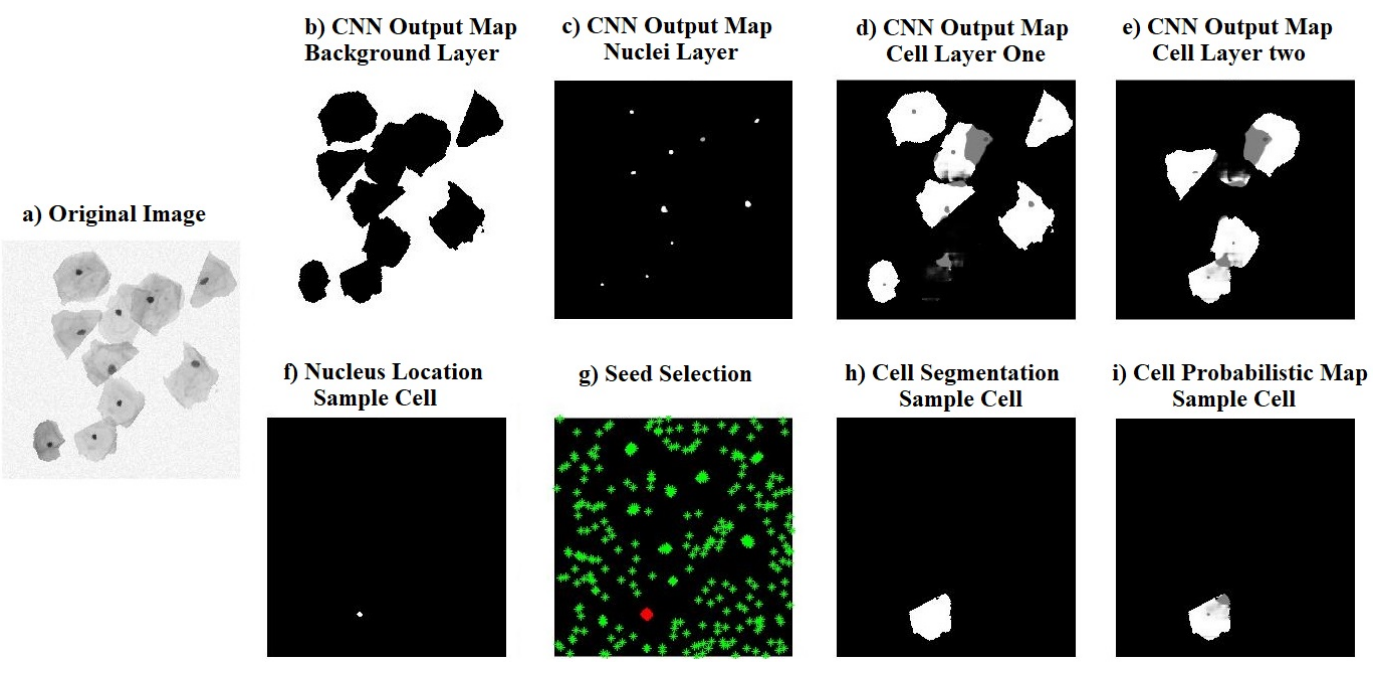

Figure 5.9: Sample images from the $C N N$ and RW processing: (a) original image, (b)-(e) the CNN output probabilistic maps for (b) background $I_{b}$, (c) cell nuclei $I_{n}$, and the two cytoplasm layers (d) $I_{c_{1}}$, (e) $I_{c_{2}}$. (f) A cell's nucleus location, (g) confident pixels as seeds for cytoplasm (red) and background (green), (h)-(i) nuclei-seeded RW segmentation output and its corresponding probabilistic map.

goal is to generate $N$ output image layers $O_{l}, l=1,2, \ldots, N$, where $O_{l}: \mathcal{R}^{2} \mapsto \mathcal{R}$ from the input image $I$, each containing one of the objects from the input image.

Having the probabilistic image maps for image $I$ from the residual $\mathrm{CNN}$ as well as the confident pixels as user input seeds $\left\{C_{l}, C_{b}\right\}$ for the nuclei location layer output from (5.6) and (5.7), we first create an image graph $G=(V, \mathcal{E})$ for the input $I$ and calculate Laplacian matrix $L$ using (3.3)(3.5). Then, we use Algorithm 1 to obtain $P r_{v_{l}}^{\ell_{k}}, v \in V$, where $P r_{v_{l}}^{\ell_{k}}$ is the probability of assigning label $\ell_{k}$ to node $v_{l}$, and $v_{l}$ is the $v^{\text {th }}$ node at layer $l$.

Applying the multi-layer random walker image segmentation method from Algorithm 1 will create a set of probabilistic image maps for the $2 D$ image layers $P r_{v_{l}}^{\ell_{k}}, l=1,2, \ldots, N$, of size $m \times$ $n \times(N+1) \times(N+1)$ with the extra +1 in the dimensions to include a background label and layer; 
each probabilistic image map belongs to one object in the original image that separates foreground (the desired object) from background in each layer. We then find the cytoplasm segmentation candidate at layer $l$ using

$$
M_{n c, l}(v)= \begin{cases}1, & \text { if } P r_{v_{l}}^{\ell_{l}}>T, \\ 0, & \text { otherwise, }\end{cases}
$$

where $T$ is a threshold value and $M_{n c, l}$ is the cytoplasm approximation using nuclei-seeded region growing at layer $l$ following applying algorithm 1. Note that (5.8) uses $P r_{v_{l}}^{\ell_{k}}$ with $k=l$ such that the $l^{\text {th }}$ label is associated with the $l^{\text {th }}$ layer. Algorithm 6 summarizes the nuclei-seeded cytoplasm approximation method.

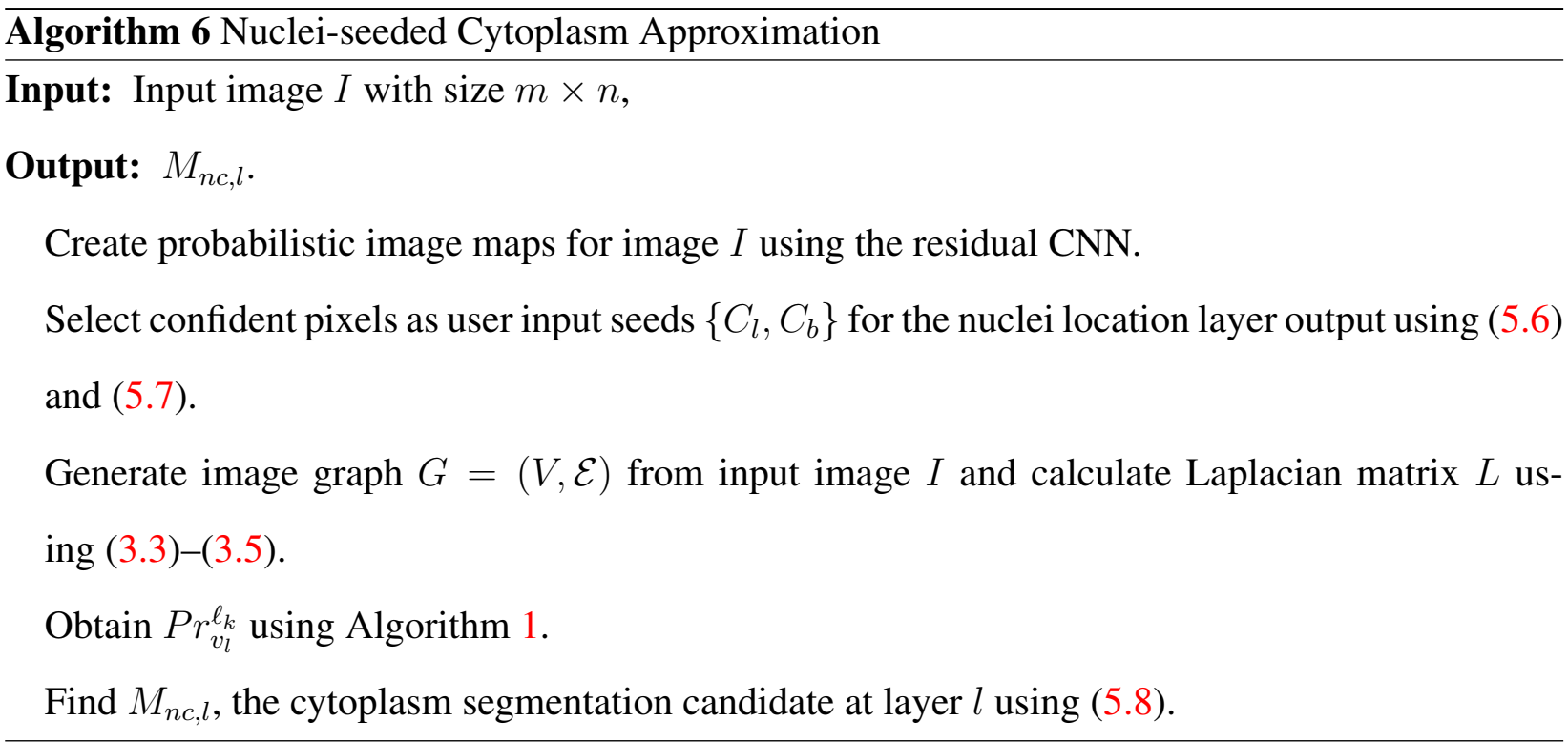

Figure 5.10 shows example cytoplasm segmentation results for the nuclei-seeded cytoplasm approximation step.

It is seen in Figure 5.10 that the cytoplasm borders are not accurate. For example, in Figure 5.10-b the segmented regions are not overlapped correctly. Also, in Figure 5.10-d some of the cells' cytoplasm are not found. 

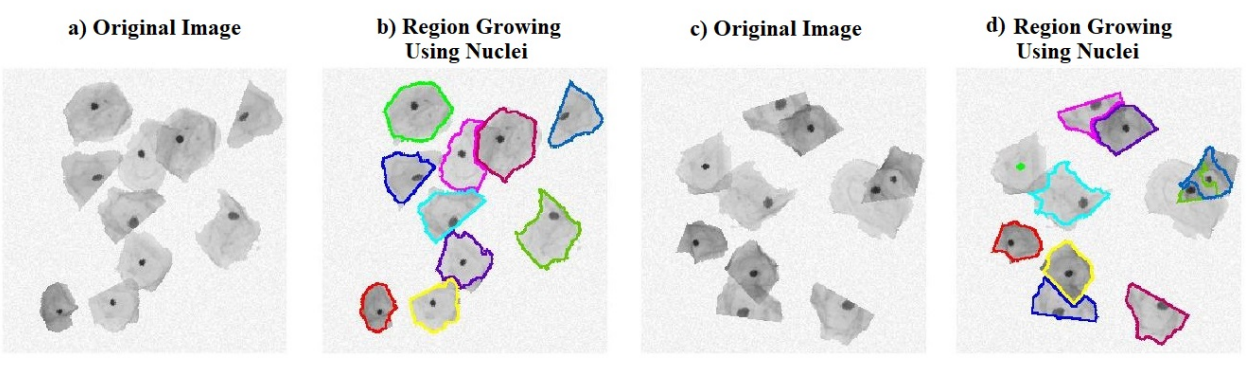

Figure 5.10: Two examples of region growing for cytoplasm approximation. a),c) Original images, b),d) segmentation results.

CNN-based Cytoplasm Approximation A morphological connected component algorithm [102] is applied to binary images $B_{i}, i \in\left\{c_{1}, c_{2}\right\}$ to find the approximate cytoplasm masks $M_{c, l}, l=1,2, \ldots, N$ where $N$ is the number of objects in the image, and the probabilistic map $P_{c}=\left\{p_{c, l} \mid l=1,2, \ldots, N\right\}$, is obtained as

$$
p_{c, l}(x)= \begin{cases}\max _{i}\left(I_{i}(x)\right), i \in\left\{c_{1}, c_{2}\right\}, & \text { if } M_{c, l}(x)=1 \\ 0, & \text { otherwise }\end{cases}
$$

where $p_{c, l}(x)$ is the probability of $l^{\text {th }}$ object at pixel $x$ at layer $l$ and $I_{i}$ represents the $i^{t h}$ layer in the CNN output map.

is the probability of the $l^{t h}$ object at pixel $v$ at layer $l$ and $I_{i}$ represents the $i^{t h}$ layer in the CNN probabilistic output map. After finding the candidate segmentation maps for the cells' cytoplasm, we fine-tune the borders of the cells. We extract additional information by merging the results of the approximate locations extracted from the $\mathrm{CNN}$ outputs and region growing using random walker image segmentation algorithm using nuclei locations as discussed in the following subsection. 


\subsubsection{Cells' Borders Fine Tuning}

With the approximate location of the $i^{t h}$ cytoplasm $M_{n c, i}$ (from nuclei-seeded region growing) and $j^{\text {th }}$ cytoplasm $M_{c, j}$ (from the CNN-based cytoplasm approximation) a matching $M$ is determined to correspond $M_{c, j}$ to $M_{n c, i}, i, j=1, \ldots, N$. We create a bipartite graph $G=(V, \mathcal{E})$ where the nodes $V$ are the masks generated by nuclei-seeded region growing and $\mathrm{CNN}$-based cytoplasm approximation, and each edge $\mathcal{E}(i, j)$ has weight $w(i, j)$ determined by

$$
w(i, j)=D\left(\text { Centroid }\left(M_{n c, i}\right), \text { Centroid }\left(M_{c, j}\right)\right)
$$

where $M_{n c, i}$ is the cytoplasm approximation of the $i^{\text {th }}$ cell using nuclei-seeded region growing, and $M_{c, j}$ is the CNN-based cytoplasm approximation of the $j^{\text {th }}$ cell using threshold on the CNN probabilistic output maps $I_{c_{1}}$ and $I_{c_{2}}, D$ is the Euclidean distance and Centroid $(I)$ is calculated by

$$
\text { Centroid }(I)=\frac{1}{|M|} \sum_{i \in I} x_{i}
$$

where $x_{i}$ is the location of $i^{t h}$ pixel in image $I$ where $I\left(x_{i}\right)=1$ and $|M|$ is the number of elements in set

$$
M=\left\{x_{i} \mid I\left(x_{i}\right)=1\right\}
$$

We then find a matching $M$ in $G$ by maximizing the matching weights in $w(i, j)$ using the Hungarian method [49] with $w(i, j)$ as the adjacency matrix. A perfect matching for $M$ is a subset of the graph edges $\mathcal{E}$ such that $\forall v \in V$ at most one edge is connected to $v$ such that $w(M)=$ $\sum_{i, j \in M} w(i, j)$ is maximized. If the cells are not overlapped, a one-to-one mapping will be found between $M_{n c, i}$ and $M_{c, j}$. However, if some parts of the cells are overlapped, the mapping results will not be unique and, therefore, some segments may not be mapped. Assume that we sort the segments after applying Hungarian method [49] such that $M_{c, j}, M_{n c, j}, j=1,2, \ldots, N_{1}$ are matching where $N_{1}<=N$. We then find the segments $M_{n c, i}, i=1,2, \ldots, N$, where no matching is found and 
correspond them to $M_{c, j}, j=N_{1}+1, \ldots, N$, as $\left(i, j_{i}^{*}\right)$ where

$$
j_{i}^{*}=\underset{j}{\operatorname{argmax}} w(i, j)
$$

We then use confident pixels in $M_{n c, i}, i=1,2, \ldots, N$ as seeds in $M_{c, j_{i}^{*}}$ and apply a random walker image segmentation algorithm to refine the cytoplasm borders in $M_{c, j_{i}^{*}}$. Algorithm 7 summarizes the cytoplasm approximation refinement using nuclei-seeded cytoplasm and CNN-based cytoplasm matching.

Algorithm 7 Cytoplasm Approximation Refinement

Input: $M_{n c, i}, \quad M_{c, j}, i, j=1,2, \ldots, N$,

Output: Border refinement for cytoplasm $M_{c, j_{i}^{*}}$

Measure the similarity between $M_{n c, i}, \quad M_{c, j}, i, j=1,2, \ldots, N$ using (5.10).

Apply Hungarian method and sort the segments to find the corresponding masks $M_{n c, j}, M_{c, j}, j=$ $1,2, \ldots, N_{1}, N_{1}<=N$.

Find the segments $M_{n c, i}, i=1,2, \ldots, N$ where no matching is found and correspond them to $M_{c, j_{i}^{*}}$ using (5.13).

Use confident pixels in $M_{n c, i}, i=1,2, \ldots, N$ as hard seeds in $M_{c, j_{i}^{*}}$ and apply Algorithm 1 to refine the cytoplasm borders in $M_{c, j_{i}^{*}}$.

Figure 5.11 shows an example where the refinement algorithm finds and segments the overlapped regions. In Figure 5.11-b, it is seen that after creating $N$-layer cervical cell segmentation masks from the CNN output, one of the output binary masks contains the mask of two cervical cells. Figure 5.11-c shows the region of interest for refinement. Applying Algorithm 7 will then create the two separated segmentation masks and tune the cell's borders (Figure 5.11-d and Figure 5.11-e).

After finding the corresponding segments, we add the information from $M_{c, j}$ into $M_{n c, j}$ and select new confident pixels obtained by CNN probabilistic output maps. Finally, we refine the cell boundaries by applying proposed random walker image segmentation algorithm using $C_{b}$ in (5.7) 
a) Original Image

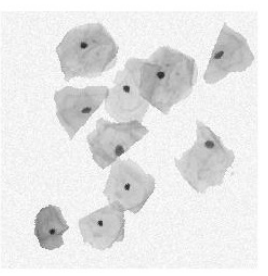

b) CNN Binary Output Map

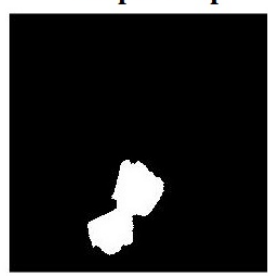

c) Refinement Region



d) First Cell

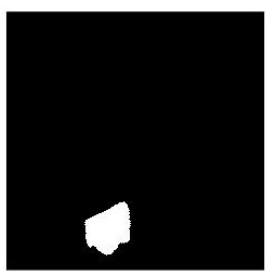

e) Second Cell

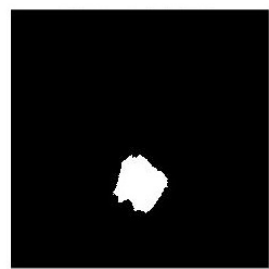

Figure 5.11: Cell segmentation results after cytoplasm refinement algorithm for one overlapped region. a) Original image, b) $\mathrm{CNN}$ binary output result, c) region of interest for refinement, d)-e) first and second cell segmentation using Algorithm 7.

and the new seed set $C_{l}$ as

$$
C_{l}=\left\{v_{l} \mid p_{c, l}\left(v_{l}\right)>T_{b}\right\}, l=1,2, \ldots, N
$$

where $p_{c, l}\left(v_{l}\right)$ is the probability map of the $l^{t h}$ cytoplasm, $M_{c, l}$, at pixel $v$ at layer $l$ and $T_{b}$ is the threshold value. In other words, for each layer $l$, we collect the pixel $v$ as foreground seed if $p_{c, l}\left(v_{l}\right)>T_{b}$ or as background seed if $P_{B}\left(v_{l}\right)>T_{b}$ for threshold value $T_{b}=0.9$. We use these pixels as seeds and run Algorithm 1 to obtain $\operatorname{Pr}_{v_{l}}^{\ell_{k}}, v \in V$, where $\operatorname{Pr}_{v_{l}}^{\ell_{k}}$ is the probability of assigning label $\ell_{k}$ to node $v_{l}$, and $v_{l}$ is the $v^{\text {th }}$ node at layer $l$.

After applying Algorithm 1, we then find the final cytoplasm segmentation at layer $l$

$$
C F_{l}\left(v_{l}\right)=P r_{v_{l}}^{\ell_{k}}>T
$$

where $T=0.8$ is the threshold value and $C F_{l}$ is the refined cytoplasm at layer $l$. Algorithm 8 summarizes the fine tuning cytoplasm segmentation method.

Figure 5.12 shows an example of our proposed three step image segmentation method. 

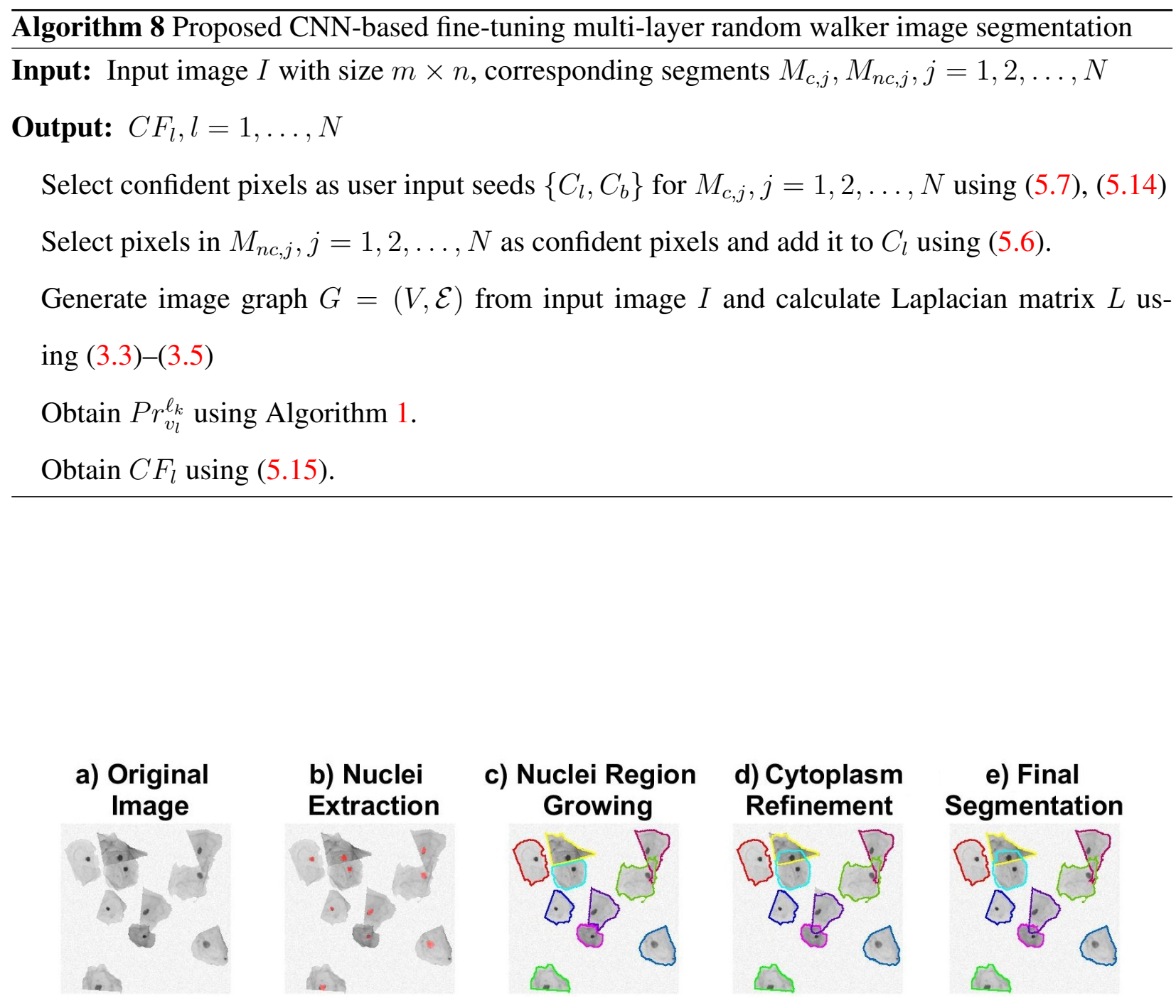

Figure 5.12: Our proposed multi-layer random walker image segmentation method. a) input image, b) nuclei locations, c) cytoplasm approximate using Algorithm 6, d) cytoplasm refinement using Algorithm 7, and e) final segmentation using Algorithm 8. 


\subsubsection{Performance Evaluation}

We synthetically manipulated, overlapped, and combined 65 individual real cervical cells to create 50000 images, each with 10 cervical cells for training our CNNs. Given the enormous number of choices for different cervical cell selection, location, and orientation, these samples represent a very small subset of all possible combinations. To evaluate performance, we applied our method on synthetic cervical cells and compared the results with [5-7].

\subsubsection{Results}

The CNN is trained over the dataset of 50000 synthetically generated images discussed in Sec. 5.2.1 with 45000 images used for training and 5000 images used for validation. Each training entry consists of an input image as the mixture of 10 cells and the output binary masks of the background layer, nuclei layer, and two cytoplasm layers. The training is done using Keras/Tensorflow over 50 epochs using a learning rate of 0.001 and the Adam optimizer.

For the multi-layer random walker image segmentation, we use empirically-selected threshold values of $t_{n}=0.2$ and $t_{i}=0.18, i \in\left\{b, c_{1}, c_{2}\right\}$ for (5.3) to create masks for finding seeds. The seeds were selected using $T_{f}=0.2$ and $T_{b}=0.9$ to find the approximate cytoplasm with $T=0.5$ and finally, the cytoplasm refinement is created using $T=0.8$. After separately training the $\mathrm{CNN}$, the entire system with all three phases was tested over 100 newly generated images and the results averaged to obtain aggregated values. Similar to the training/validation images, the test images are synthetically generated from real cells in the ISBI 2014 dataset; therefore, the ground truth segmentation of the background, nuclei, and cytoplasm masks are available.

Figure 5.13 shows an example of cell segmentation results after cytoplasm refinement, including the original image, the nuclei extraction step, the initial cell cytoplasm region growing results using Algorithm 6, the results of the refinement steps using Algorithms 7 and 8, and the ground truth for comparison. 

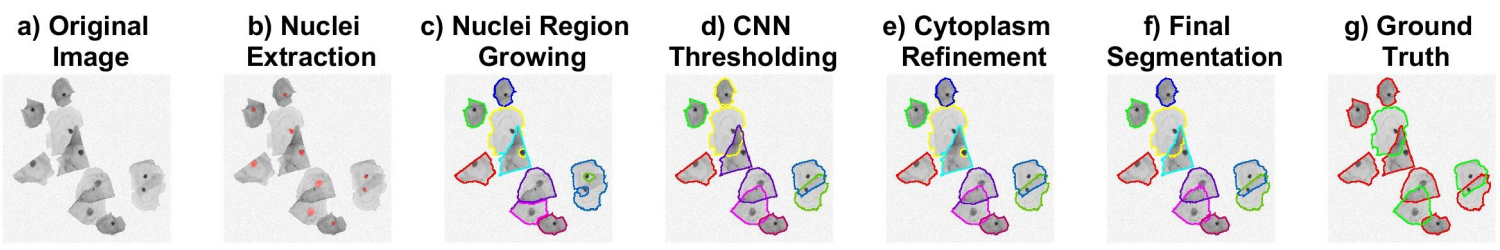

Figure 5.13: Example experimental result for the proposed three-step CNN-based image segmentation method. a) input image, b) nuclei location extraction, c) nuclei-seeded region growing using Algorithm 6, d) cytoplasm candidates using thresholding, e) cytoplasm refinement using Algorithm 7, f) final cytoplasm segmentation using Algorithm 8, and g) is the ground truth. The information from cytoplasm candidates improved the final segmentation results. For cells that cytoplasm candidates were not useful, the results from nuclei-seeded region growing was used for the final segmentation results. For the segmentation in e) compared to the ground truth, the segmentation performance in f) is $A c c=0.9996, D S C=0.9939$, $F N R=0.0042, T P R=0.9958$, and $F P R=0.0002$. 
a) Ground Truth

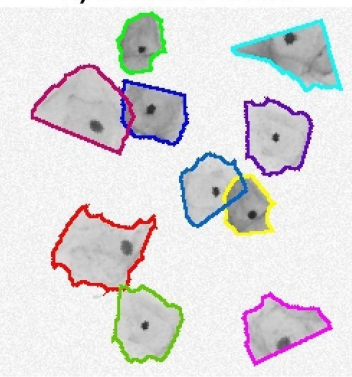

d) Method in [6]

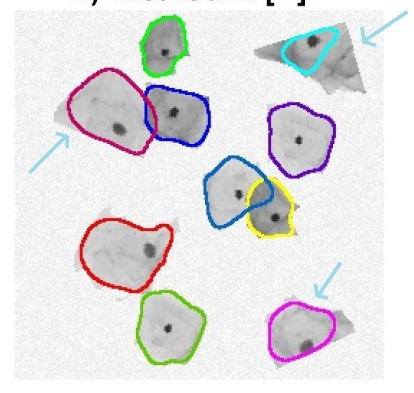

b)

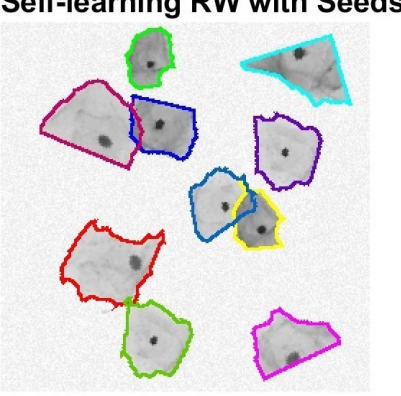

e) Method in [7]

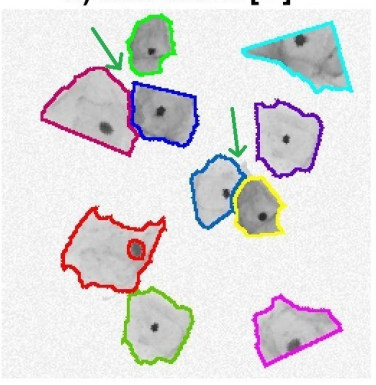

c) Method in [5]

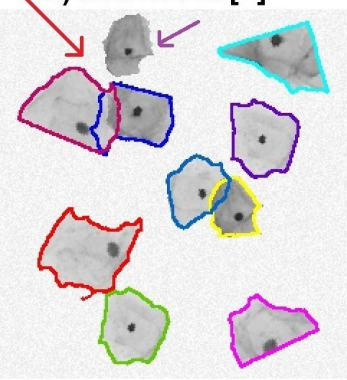

f) Method in [4]



Figure 5.14: Comparing image segmentation results. a) is the ground truth image segmentation, b) our proposed three step method, compared to three c)-e) graph-based image segmentation methods [5-7], f) three-step CNN-based image segmentation method [4].

Figure 5.14 compares our method to three different graph-based image segmentation methods and one CNN-based image segmentation method for multiple cervical cells [4-7]. Arrows in Figure 5.14 show where other methods fail to segment the cells correctly. 
We evaluated our segmentation results quantitatively by

$$
\begin{aligned}
& A c c=\frac{T P+T N}{T P+T N+F P+F N} \\
& D S C=\frac{2 * T P}{2 * T P+F P+F N} \\
& T P R=\frac{T P}{T P+F N} \\
& F N R=\frac{F N}{F N+T P} \\
& F P R=\frac{F P}{F P+T N}
\end{aligned}
$$

where $A c c$ is the accuracy, $D S C$ is the dice score measure, $T P R, F N R, F P R$ are the true positive rate, false negative rate, and false positive rate and

- TP: (True Positive) is the number of foreground pixels correctly segmented at each output image layer,

- TN: (True Negative) is the number of background pixels correctly segmented at each output image layer,

- FP: (False Positive) is the number of background pixels segmented as foreground at each output image layer,

- FN: (False Negative) is the number of foreground pixels segmented as background at each output image layer.

Table 5.1 shows the segmentation quantitative results. We compared our proposed method to three different graph-based image segmentation methods and one CNN-based method for multiple cervical cells [4-7]. The results are averaged over 100 synthetic jointly overlapped cervical cell images.

In Table 5.1, mean and std represent the average and standard deviation values of the measures. For simplicity to compare different methods, the best value for each segmentation quantitative measurement is represented as the bold font. 
Table 5.1: Segmentation quantitative measure.

\begin{tabular}{|c|c|c|c|c|c|c|c|c|c|c|}
\hline \multirow{2}{*}{ Description } & \multicolumn{2}{|c|}{ Acc } & \multicolumn{2}{|c|}{ DSC } & \multicolumn{2}{|c|}{ FNR } & \multicolumn{2}{|c|}{ TPR } & \multicolumn{2}{|c|}{ FPR } \\
\hline & mean & std & mean & std & mean & std & mean & std & mean & std \\
\hline Proposed Method & 0.995 & 0.006 & 0.932 & 0.061 & 0.021 & 0.024 & 0.979 & 0.024 & 0.005 & 0.006 \\
\hline Lu et al. [5] & 0.993 & 0.004 & 0.897 & 0.050 & 0.072 & 0.056 & 0.928 & 0.056 & 0.005 & 0.003 \\
\hline $\begin{array}{c}\text { Phoulady et al. } \\
\text { [6] }\end{array}$ & 0.993 & 0.003 & 0.882 & 0.044 & 0.187 & 0.063 & 0.813 & 0.063 & 0.0009 & 0.0008 \\
\hline Sung et al. [7] & 0.974 & 0.018 & 0.733 & 0.112 & 0.042 & 0.055 & 0.958 & 0.055 & 0.026 & 0.019 \\
\hline Wan et al. [4] & 0.978 & 0.006 & 0.592 & 0.126 & 0.500 & 0.133 & 0.500 & 0.133 & 0.006 & 0.004 \\
\hline
\end{tabular}

Because our proposed method is a three-step algorithm, the results of the segmentation are dependent on the results of the CNN probabilistic map. For situations where the nuclei of the cells are close to the overlap in the input image, the CNN probabilistic map merges the two cells into one single cell. However, because one of our CNN output layers locates the nuclei of cells, we use a nuclei-based random walker image segmentation method to find a rough estimate of the individual cells. In $[5,6]$, the authors first find the nucleus and then they use this information to find the cell boundaries. It is seen in Figure 5.14 that comparing our proposed method to [4-7], our method gives better cell segmentation results, especially on the cells that have close nuclei in the input image where in the other methods the cell boundaries are merged together in the segmentation output in those areas. Although the methods in [5,6] give better results comparing to [4,7], they don't use offline training, and therefore, these methods are very time consuming. The authors 
in [7] use super pixel information as hard seeds. We provide these hard seed information from our offline CNN-based training, so results of [7] are close to our nuclei-based region growing step. However, this method will give very poor results if the seeds are not selected correctly. Similar to our proposed method, the authors in [4] use a CNN-based method followed by a fine tuning method for cervical cell segmentation. Therefore, the method in [4] is faster than [5] because of the offline training. It also gives better results than [7] where both use offline training. However, because of using the DRLSE package for border refinement, the method in [4] gives poor results when the image mixture has regions with homogeneous image intensity values comparing to our proposed method. Table 5.2 gives the training and test time complexity for different methods. The training time used for the proposed method and the method in [7] is over 5000 images. The test is the average time to segment one image for all methods. The execution time is averaged over 100 images. The training step simulations are done using python platform on SharcNet cluster [103] and the test for all the simulations are done using Matlab platform on Windows 10 Intel $i 7$ CPU 2.2 GHz.

\subsection{Multiple Overlapped Cervical Cell Segmentation}

Similar to the previous section, a dataset with ground truth labels are provided as the training samples for CNN, each training sample containing the input image with the binary images of background layer, nuclei layer, and non-overlapping cytoplasm layers.

\subsubsection{Data Set-up}

In the jointly overlapped cervical cell dataset, each image consist of 10 individual cervical cells and the maximum overlap of cells in any region of the image is 2 . However, in real images, the cervical cells can have more overlap and the number of cells in the image can vary. Therefore, 
Table 5.2: Training and test time complexity.

\begin{tabular}{|c|c|c|}
\hline Description & Offline training (in hours) & Test (in sec) \\
\hline Proposed Method & 24.4 & $\mathbf{1 . 2 7}$ \\
\hline Lu et al. [5] & -- & 349.45 \\
\hline Phoulady et al. [6] & -- & 3.53 \\
\hline Sung et al. [7] & 24.6 & 13.74 \\
\hline Wan et al. [4] & 25.9 & 19.53 \\
\hline
\end{tabular}

we created a more complex synthetic cervical cell dataset to extend our work and applied a slightly different segmentation method for this multiple overlapped cervical cell dataset. Also by increasing the number of individual cervical cells, a very small subset of all possible combinations was created for our multiple overlapped cervical cell dataset.

Similar to the previous dataset, each training sample contains the input image with the binary images of background layer, nuclei layer, and non-overlapping cytoplasm layers. Unlike the previous dataset, images have varied number of cervical cells and have maximum overlap of four; therefore, each image contains four non-overlapping cytoplasm layers instead of two.

The training and test image datasets were created from 573 individual cervical cells extracted from the ISBI 2014 Overlapping Cervical Cytology Image Segmentation Challenge dataset [15]. Random rotations and translations on these extracted cells were performed to increase variation in the cell sample population. Then, synthetic images were created by overlapping and combining these images of cervical cells using the Beer-Lambert law [14]. Figure 5.15 shows a few individual 


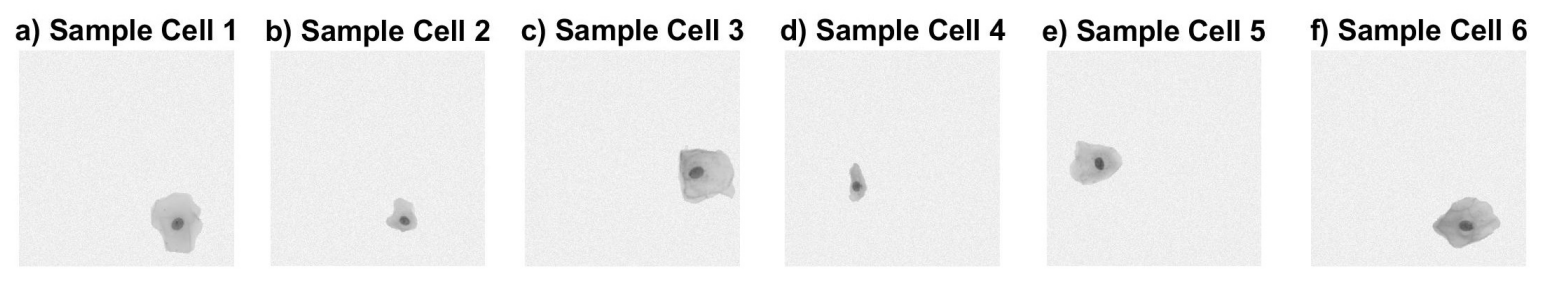

Figure 5.15: A few samples of individual cervical cells from the $I S B I 2014$ Overlapping Cervical Cytology Image Segmentation Challenge dataset [15] to create multiple overlapped cervical cell dataset using Beer-Lambert Law [14].

cervical cell images from the ISBI2014 dataset [15].

Following the approach in 5.2.1, we generated 100000 synthetic images by overlapping and combining real cervical cell images using (5.2). Images were produced with 2, 3, 5, 7, or 10 cervical cells and any particular region $x$ has a maximum overlap of four cells (i.e., $\left.\forall x, N_{o}(x) \leq 4\right)$. To determine $E_{B}$ in (5.2), we averaged over the background area for all the training images in the ISBI 2014 dataset [15]. Also, the ordered set [0.95, 0.9, 0.85, 0.8] was used for $\alpha_{i}(x)$ for $N_{o}(x) \leq 4$ as the coefficients to control the difference of the thickness in the overlapping area. We rotated and translated the cells from the ISBI 2014 dataset [15] and the percentage of overlap for cells in each image was randomly selected. Figure 5.16 shows a few synthetic image mixtures created using this approach.

Since the images in the dataset are synthetically generated, each training and test image consists of an input image $I$ and binary masks of the background layer $B_{b}$, nuclei layer $B_{n}$, and each of the cytoplasm layers, $B_{c_{1}}, B_{c_{2}}, B_{c_{3}}$, and $B_{c_{4}}$ where each $B_{c_{i}}$ contains no overlap but their combination can result in a maximum of $N_{o}(x)=4$.

Algorithm 9 explains generating our dataset. 


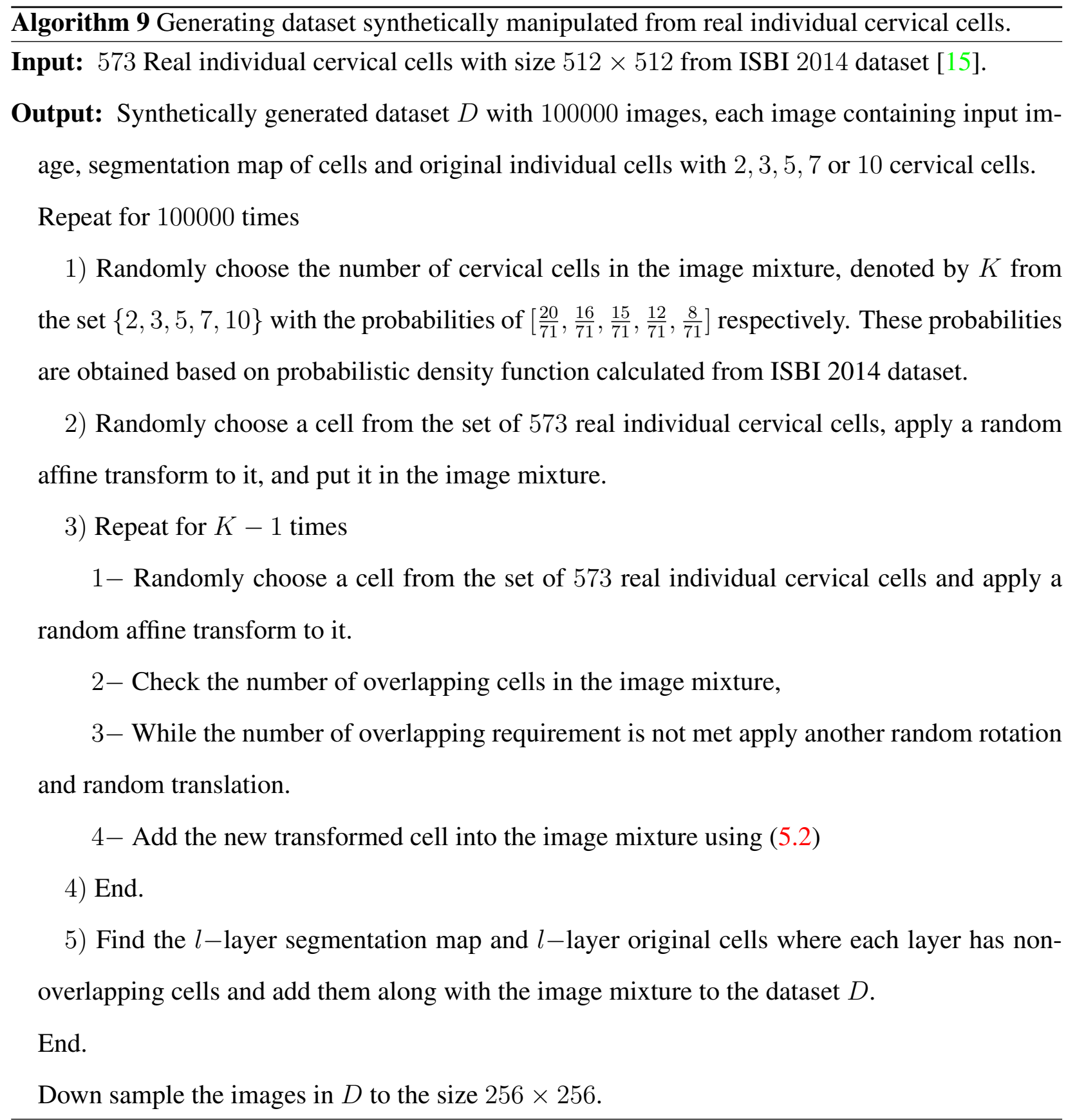



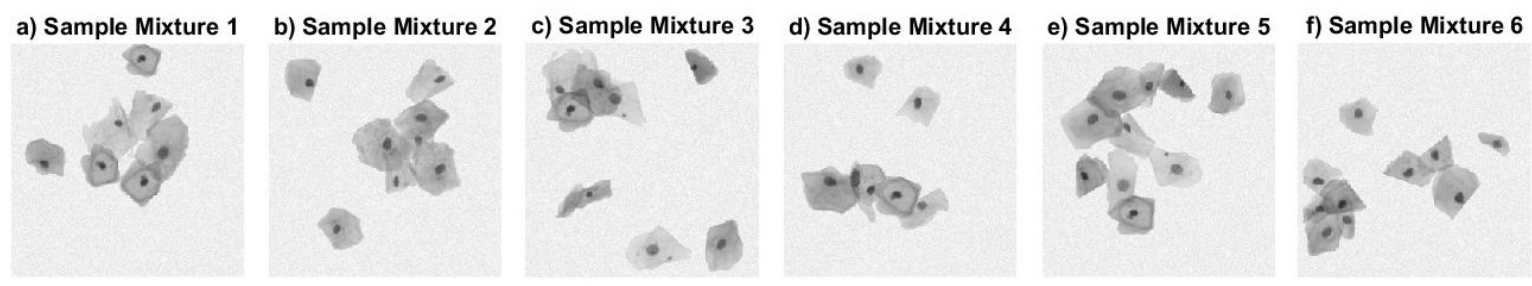

Figure 5.16: Example synthetic image mixtures from the overlapped cervical cell dataset created using (5.2).

\subsubsection{Nucleus and Cytoplasm Mapping}

At the second step of the proposed method, we take the probability image maps from the residual CNN for the background $\left(I_{b}\right)$, cell nuclei $\left(I_{n}\right)$, and the four cell cytoplasm layers $\left(I_{c_{1}}, I_{c_{2}}, I_{c_{3}}\right.$, and $I_{c_{4}}$ ) and use these to generate an $N$-layer probabilistic image where each layer contains the map of individual segmented cells, where $N$ is the number of objects in $I_{n}$. The steps taken are outlined in the following paragraphs.

\subsubsection{Nuclei and Approximate Cytoplasm Detection}

We first find the location of the nucleus for each cell and the approximate location of the cells' cytoplasm by using a threshold $t_{i}$ on the CNN output probabilistic maps using

$$
B_{i}= \begin{cases}1, & \text { if } I_{i}>t_{i}, i \in\left\{b, n, c_{1}, c_{2}, c_{3}, c_{4}\right\} \\ 0, & \text { otherwise }\end{cases}
$$

where $B_{i}$ is a binary image containing the approximate location of the background, nuclei or cytoplasm, and $t_{i}$ is a threshold value for layer $i$. We then apply the morphological connected component algorithm [102] on $B n$ to create an $N$ layer nuclei masks $M_{n, l}, l=1,2, \ldots, N$, where $N$ is the 
number of detected nuclei in $B n$. Having the binary masks $M_{n, l}, l=1,2, \ldots, \ldots, N, B_{b}$ we then select the seeds and apply a multi-layer random walker image segmentation algorithm [45] to extract the approximate cytoplasm candidates. The details are explained in the following paragraphs.

Nuclei-based Seed Selection Similar to the previous section, we find the foreground and background confident pixel sets $C_{l}, l=1,2, \ldots, N$, and $C_{B}$.

Proposed Nuclei-based Multi-layer Random Walker Image Segmentation After selecting the seeds, we run Algorithm 6 to extract the $N$-layer approximate cytoplasm candidates, $M_{n c, l}, l=$ $1,2, \ldots, N$, where $N$ is the number of cervical cells in the image.

Figure 5.17 shows the probabilistic output map of the CNN, seed selection, nuclei-based cytoplasm approximate and final cell segmentation for a sample cell.

CNN-based Cytoplasm Approximation Similar to the previous section, a morphological connected component algorithm [102] is applied to binary images $B_{i}, i \in\left\{c_{1}, c_{2}, c_{3}, c_{4}\right\}$ to find the approximate cytoplasm masks $M_{c, l}, l=1,2, \ldots, N$ where $N$ is the number of objects in the image, and the probabilistic map $P_{c}=\left\{p_{c, l} \mid l=1,2, \ldots, N\right\}$, is obtained as

$$
p_{c, l}(x)= \begin{cases}\max _{i}\left(I_{i}(x)\right), i \in\left\{c_{1}, c_{2}, c_{3}, c_{4}\right\}, & \text { if } M_{c, l}(x)=1 \\ 0, & \text { otherwise }\end{cases}
$$

where $p_{c, l}(x)$ is the probability of $l^{\text {th }}$ object at pixel $x$ at layer $l$ and $I_{i}$ represents the $i^{\text {th }}$ layer in the CNN output map. After finding the candidate segmentation maps for cells' cytoplasm, we fine-tune the borders of the cells. We extract additional information by merging the results of the approximate locations extracted from the $\mathrm{CNN}$ outputs and region growing using random walker image segmentation algorithm using nuclei locations. 


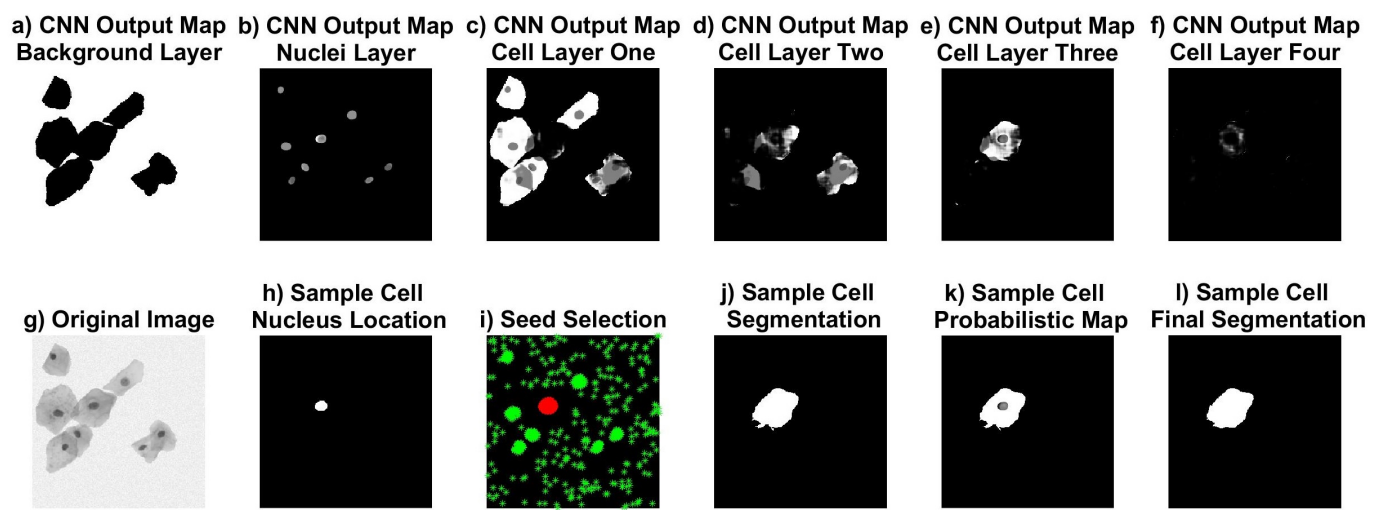

Figure 5.17: Approximating cells' cytoplasm with a)-f) the CNN output probabilistic maps for (a) background $I_{b}$, (b) cell nuclei $I_{n}$, and the four cytoplasm layers (c) $I_{c_{1}}$, (d) $I_{c_{2}}$, (e) $I_{c_{3}}$, (f) $I_{c_{4}}$ f)-i) proposed method to approximate the cytoplasm. g) original image, h) A cell's nucleus location, i) confident pixels as seeds for cytoplasm (red) and background (green), j)k) nuclei-based RW segmentation output and its corresponding probabilistic map and 1) final segmentation for the sample cell. The results from j), k) and c)-f) are conditionally merged to find final segmentation at 1$)$.

\subsubsection{Cells' Borders Fine Tuning}

With the approximate location of the $i^{t h}$ cytoplasm $M_{n c, i}$ (from nuclei-seeded region growing) and $j^{\text {th }}$ cytoplasm $M_{c, j}$ (from the CNN-based cytoplasm approximation) a matching $M$ is determined to correspond $M_{c, j}$ to $M_{n c, i}, i, j=1, \ldots, N$. We create a bipartite graph $G=(V, \mathcal{E})$ where the nodes $V$ are the masks generated by nuclei-seeded region growing and $\mathrm{CNN}$-based cytoplasm approximation, and each edge $\mathcal{E}(i, j)$ has weight $w(i, j)$ determined by

$$
w(i, j)=\left|M_{n c, i} \wedge M_{c, j}\right|
$$

where $\wedge$ is the logical AND operator, $\left|M_{n c, i} \wedge M_{c, j}\right|$ is the number of pixels in $M_{n c, i} \wedge M_{c, j}, M_{n c, i}$ is the cytoplasm approximation of the $i^{t h}$ cell using nuclei-seeded region growing, and $M_{c, j}$ is 
the CNN-based cytoplasm approximation of the $j^{\text {th }}$ cell using threshold on the CNN probabilistic output maps $I_{c_{1}}, I_{c_{2}}, I_{c_{3}}$, and $I_{c_{4}}$. We then run Algorithm 7 to refine the cytoplasm borders in $M_{c, j_{i}^{*}}$, where $j_{i}^{*}$ is obtained by (5.13).

Although the cytoplasm segmentation using nuclei-seeded region growing $M_{n c, j}$ gives a good estimate of the location of the cell, it does not include the overlapped parts. Therefore, after finding the one-to-one matching between $M_{c, j}$ and $M_{n c, j}, j=1,2, \ldots, N$, we include information from $M_{c, j}$ to $M_{n c, j}$ to refine the edge boundaries of the cells using $M_{n c, j} \vee M_{c, j}$, where $j=1,2, \ldots, N$ is the mask layer and $\vee$ is the logical OR operator.

Also, since $M_{c, j}$ might not provide perfect segmentation, we check if the included information is useful or not. We measure the similarity of $M_{n c, j}$ and $M_{n c, j} \vee M_{c, j}$ by computing the sample Pearson correlation coefficient

$$
r_{j}=\operatorname{corr}\left(M_{n c, j}, M_{n c, j} \vee M_{c, j}\right)
$$

for each cell cytoplasm $j$. If the correlation $r_{j}$ is greater than some threshold $T_{c}$, the results of the fine tuning to obtain $M_{n c, j} \vee M_{c, j}$ are kept, otherwise the nuclei-seeded region growing segmentation $M_{n c, j}$ results

$$
C F_{j}= \begin{cases}M_{n c, j} \vee M_{c, j}, & \text { if } r_{j}>T_{c} \\ M_{n c, j}^{T=0.1 \cdots 0.5} \vee M_{c, j}, & \text { if }\left(r_{j} \leq T_{c}\right) \wedge\left(\bar{r}_{j}>T_{c}\right) \\ M_{n c, j} & \text { if }\left(r_{j} \leq T_{c}\right) \wedge\left(\bar{r}_{j} \leq T_{c}\right)\end{cases}
$$

where

$$
\bar{r}_{j}=\operatorname{corr}\left(M_{n c, j}^{T=0.1 \cdots 0.5}, M_{n c, j}^{T=0.1 \cdots 0.5} \vee M_{c, j}\right),
$$

$C F_{j}, j=1,2, \ldots, N$ are the final cytoplasm segmentation masks, $T_{c}=0.85$ and $M_{n c, j}^{T=0.1 \cdots 0.5}$ is calculated using Algorithm 10.

Figure 5.18 illustrates two examples of correlation of $M_{n c, j}$ and $M_{n c, j} \vee M_{c, j}$ with high and low correlation $r_{j}$. Figure 5.19 shows an example where the fine tuning algorithm modifies the regions. 


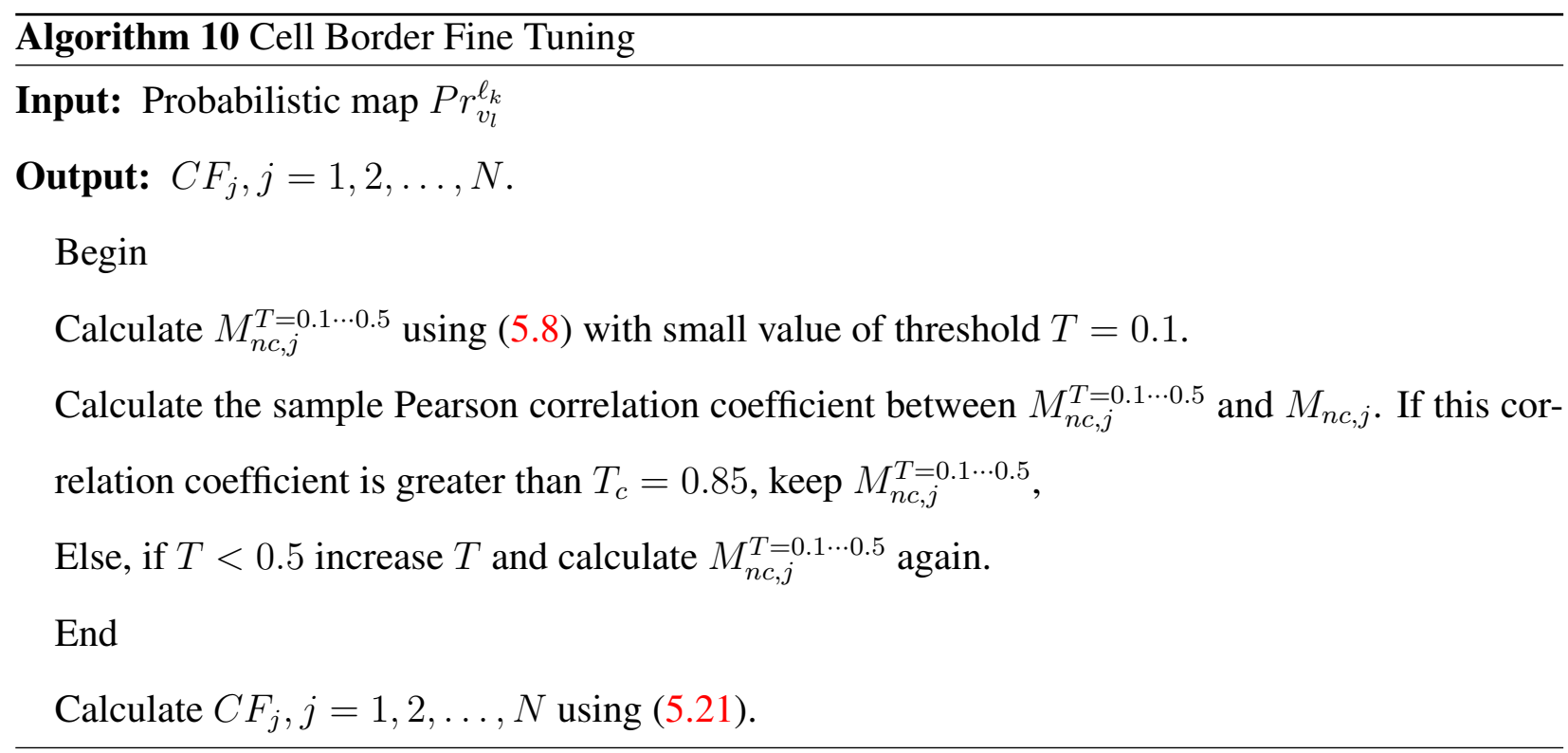

In Fig. 5.19, the top row shows an example when improved segmentation occurs by including the CNN thresholding segmentation results. The bottom row shows an example when including the extra information from the $\mathrm{CNN}$ thresholding to the nuclei-seeded region growing cytoplasm segmentation does not improve the segmentation results and, therefore, we discard including the information.

Figure 5.20 shows an example of cell segmentation results after cytoplasm refinement, including the original image, the nuclei extraction phase, the initial cell cytoplasm region growing results, the results of the refinement stage, and then the ground truth for comparison. It should be noted that the refinement stage in Fig. 5.20 has corrected the segmentation for a number of the overlapping regions for the cells, particularly with the cluster of five cells in the bottom-right of the image.

\subsubsection{Performance Evaluation}

To evaluate the proposed method, we applied the method on synthetic cervical cells and compared the results with [4-7]. Lu et al. [5] proposed multiple level set functions to address overlapping cervical cells. In [6], extracted nuclei locations were used to segment multi-layer overlapping 



Figure 5.18: Visualization for correlation of $M_{n c, j}$ and $M_{n c, j} \vee M_{c, j}$ : a) high correlation, b) low correlation.

cervical cells. Authors in [4] created a CNN-based multi-step approach for overlapping cervical cell segmentation, and in [7], the authors used a superpixel partitioning for segmentation.

\subsubsection{Results}

The CNN is trained over the dataset of 100000 synthetically generated images discussed in Sec. 5.3.1 with 90000 images used for training and 10000 images used for validation. Each training entry consists of an input image and the output binary masks of the background layer, nuclei layer, and four cytoplasm layers. The training is done using Keras/Tensorflow over 50 epochs using a learning rate of 0.001 and the Adam optimizer.

For the multi-layer random walker image segmentation, we use the thresholds $t_{n}=0.2$ and $t_{i}=0.2, i \in\left\{b, c_{1}, c_{2}, c_{3}, c_{4}\right\}$ for (5.17) to create masks for finding seeds. The seeds were selected using $T_{f}=0.2$ and $T_{b}=0.9$ to find the approximate cytoplasm with $T=0.5$ and finally, the cytoplasm refinement is created using $T_{c}=0.85$ and all the threshold values are empirically selected. After separately training the $\mathrm{CNN}$, the entire system with all three phases was tested over 


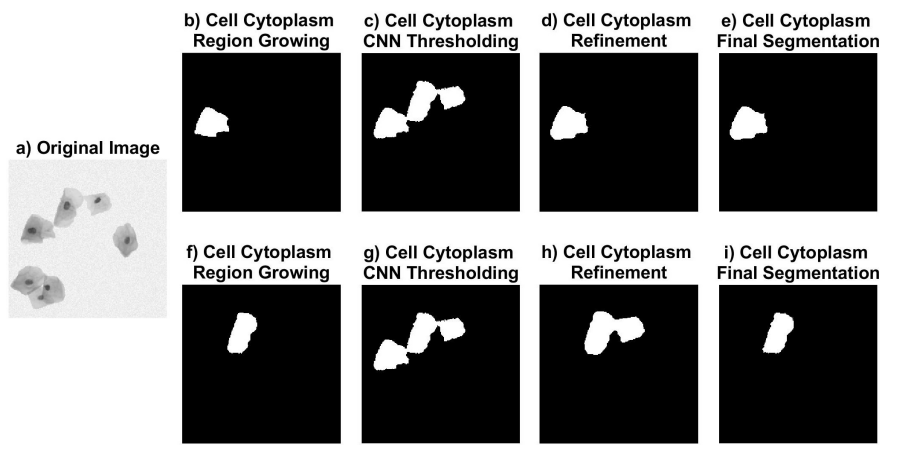

Figure 5.19: Cell segmentation results after cytoplasm refinement algorithm. a) Original image, b),f) nuclei-based cell cytoplasm, c),g) CNN-based cell cytoplasm, d),h) CNN-based cell refinement using Algorithm 7, e),i) final cell segmentation using Algorithm 10.

100 newly generated images and the results averaged to obtain aggregated values. Similar to the training/validation images, the test images are synthetically generated from real cells in the ISBI 2014 dataset; therefore, the ground truth segmentation of the background, nuclei, and cytoplasm masks are available.

Figure 5.21 shows an example of intermediate and final results for the proposed three phase image segmentation method. It is seen in Fig. 5.21 that when the cytoplasm candidates calculated from CNN segmentation in Fig. 5.21(d) are not useful to improve the final results, the results from nuclei-seeded region growing in Fig. 5.21(c) are used as the final segmentation results. However, when cytoplasm candidates in Fig. 5.21(e) are useful, they are used to refine the cell borders to obtain Fig. 5.21(f).

A comparison of the proposed method to four other techniques is shown in Fig. 5.22 with the ground truth segmentation shown in Fig. 5.22(a). Example results from the proposed method is shown in Fig. 5.22(b), while results for three different graph-based methods are shown in Fig. 5.22(c)-(e) [5-7] and to one CNN-based cervical cell segmentation method in Fig. 5.22(f) [4]. The arrows in Fig. 5.22 highlight areas where the methods fail to segment the cells correctly. 

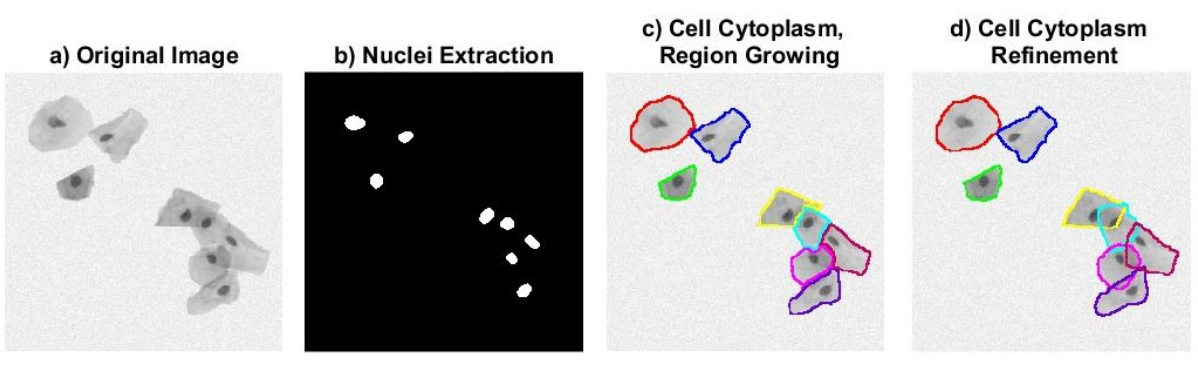

e) Ground Truth

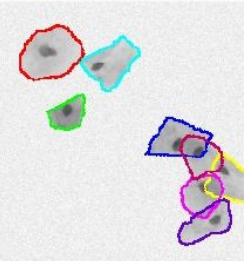

Figure 5.20: Our proposed multi-layer random walker image segmentation method. a) input image, b) nuclei locations, c) nuclei-seeded random walker image segmentation for region growing, d) segmentation results using region refinement and e) ground truth.
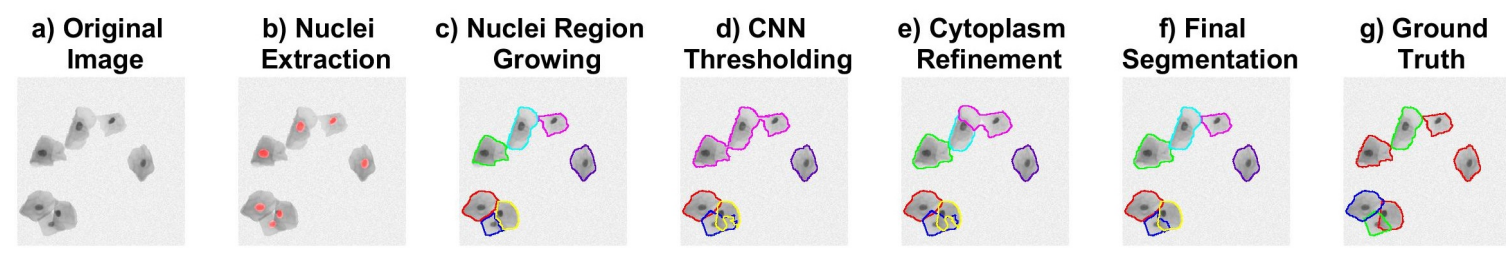

Figure 5.21: Example experimental result for the proposed three-step CNN-based image segmentation method. a) input image, b) nuclei location extraction, c) nuclei-seeded region growing using multi-layer random walker image segmentation, d) cytoplasm candidates using thresholding, e) cytoplasm refinement using Algorithm 7, f) final cytoplasm segmentation using Algorithm 10, and g) is the ground truth. The information from cytoplasm candidates improved the final segmentation results. For cells that cytoplasm candidates were not useful, the results from nuclei-seeded region growing was used for the final segmentation results. For the segmentation in e) compared to the ground truth, the segmentation performance in $\mathrm{f}$ ) is $A c c=0.998, D S C=0.968, F N R=0.021, T P R=0.979$, and $F P R=0.001$. 


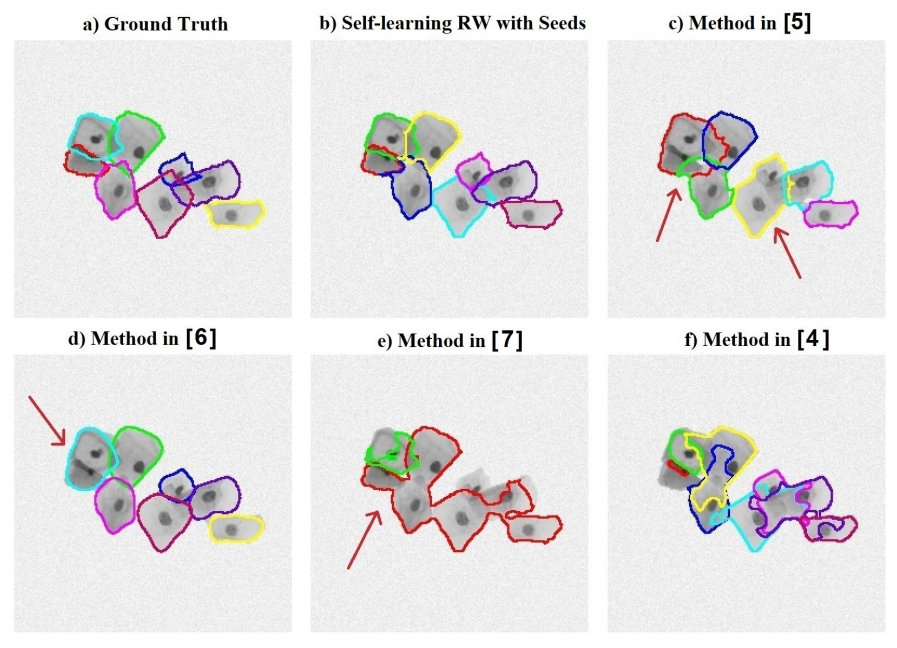

Figure 5.22: Comparing image segmentation results. a) is the ground truth image segmentation, b) the proposed three stage method, compared to three c)-e) graph-based image segmentation methods [5-7], f) three-step CNN-based image segmentation method [4].

It is seen in Fig. 5.22 that the proposed method finds cell borders correctly while the competing methods give a rough estimate of the borders. Also in Fig. 5.22, the other methods fail to find the correct number of cells while the proposed method finds the number of the cells correctly and also the borders for cells are close to the ground truth.

Since the proposed method is a three-step algorithm, the results of the segmentation are dependent on the results of the CNN probabilistic image maps. For situations where the nuclei of the cells are close to each other, the CNN probabilistic image map merges the two cells into one single cell. However, because one of the $\mathrm{CNN}$ output layers locates the nuclei of cells, we use the nuclei-seeded random walker image segmentation method to still find a rough estimate of the individual cells. It is seen in Fig. 5.22 that the proposed method gives better cell segmentation results, especially on cells that have close nuclei. For example, the two cells on the left side of each image in Fig. 5.22 with close nuclei are better segmented with the proposed method than with the other methods. 
In [4-6], the authors first find the nucleus and then use this information to find the cell boundaries. Although the methods in [5,6] give better results compared to [7], they don't use offline training, and therefore, these methods are very time consuming while [7] uses offline training. Authors in [7] use super pixel information as hard seeds. This seed information is provided from the offline CNN-based training, so results of [7] are close to the nuclei-seeded region growing step in the proposed method. However, this method will give very poor results if the seeds are not selected correctly. Similar to our proposed method, the authors in [4] use a CNN-based method followed by a fine tuning method for cervical cell segmentation. Therefore, similar to the proposed method, the method in [4] is faster than [5] because of the offline training. It also gives better results than [7] where both use offline training. However, because of using the DRLSE package for border refinement, the method in [4] gives poor results when the image mixture has regions with homogeneous image intensity values comparing to our proposed method.

Table 5.3 shows quantitative segmentation results for accuracy (Acc), dice score (DSC), false negative rate (FNR), true positive rate (TPR), and false positive rate (FPR). The mean and standard deviation (std) results are based on 100 synthetic overlapped cervical cell image mixtures and the bold font shows the best result for each measure. It is seen from Table 5.3 that the proposed method outperforms the other four methods for nearly all quantitative measures.

Table 5.4 gives the training and test times for the different methods. The training time used for the proposed method and the method in [4,7] is over 90000 images. The test time is the average time to segment one image for all methods, averaged from a run over 100 images. The training of the CNN is done using Keras/Tensorflow on the SharcNet cluster [103] (with 2 GPUs, each having 4 CPUs and 15 Gigabytes memory), while the full three-step segmentation is done using Matlab under Windows 10 Intel $i 7$ CPU $2.2 \mathrm{GHz}$.

It is worth noting that due to lack of access to labeled real data, only synthetic data are used to train the neural networks and to evaluate the performance of the proposed algorithms. Although 
Table 5.3: Aggregate mean and standard deviation quantitative segmentation results for the proposed method and those in [4-7] based on a set of 100 test images.

\begin{tabular}{|c|c|c|c|c|c|c|c|c|c|c|}
\hline \multirow[t]{2}{*}{ Description } & \multicolumn{2}{|c|}{ Acc } & \multicolumn{2}{|c|}{ DSC } & \multicolumn{2}{|c|}{ FNR } & \multicolumn{2}{|c|}{ TPR } & \multicolumn{2}{|c|}{ FPR } \\
\hline & mean & std & mean & std & mean & std & mean & std & mean & std \\
\hline Proposed Method & 0.999 & 0.001 & 0.972 & 0.039 & 0.028 & 0.043 & 0.972 & 0.043 & 0.000 & 0.001 \\
\hline Lu et al. [5] & 0.997 & 0.003 & 0.887 & 0.136 & 0.089 & 0.141 & 0.911 & 0.141 & 0.002 & 0.003 \\
\hline $\begin{array}{c}\text { Phoulady et al. } \\
\text { [6] }\end{array}$ & 0.998 & 0.002 & 0.932 & 0.047 & 0.099 & 0.066 & 0.901 & 0.066 & 0.000 & 0.000 \\
\hline Sung et al. [7] & 0.995 & 0.010 & 0.861 & 0.149 & 0.106 & 0.101 & 0.894 & 0.101 & 0.004 & 0.010 \\
\hline Wan et al. [4] & 0.996 & 0.006 & 0.887 & 0.096 & 0.085 & 0.037 & 0.915 & 0.037 & 0.003 & 0.005 \\
\hline
\end{tabular}

the synthetic data are carefully designed to mimic the real cervical cell images and follow the same statistical properties, it cannot fully replace the real data for training and testing the algorithm. To be used for cell segmentation of real cervical cell images, the neural networks and subsequent algorithms should be trained and fine tuned with an extensive set of labeled real data. With access only to a few real cervical cell images with composition similar to those of synthetic data used for training the algorithms, such a task is impossible. However, to simply illustrate how the proposed algorithms with synthetic training data are generalized to real data, they are applied to some real cervical cell images. Similar to the simulation results on synthetic data, each image set in Figures 5.23-5.26 illustrates one original image and the segmentation results on that image with our proposed method and four competing ones in [4-7]. 
a) Original Image

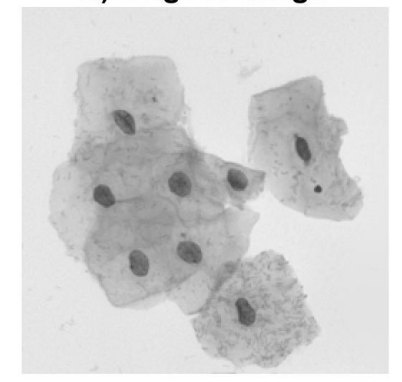

d) Method in [6]

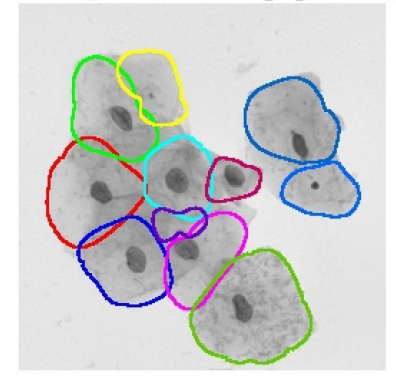

b) Self-learning RW with Seeds

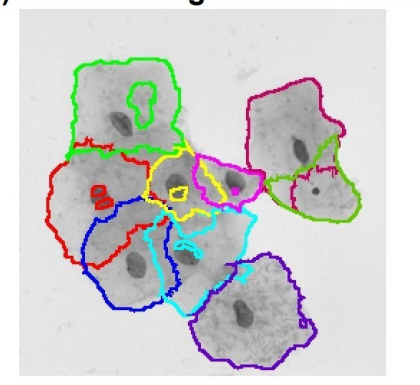

e) Method in [7]

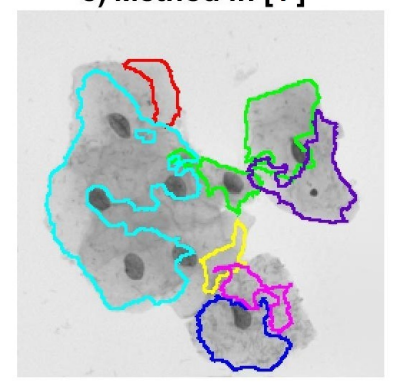

c) Method in [5]

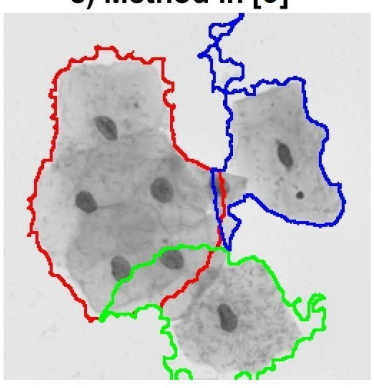

f) Method in [4]

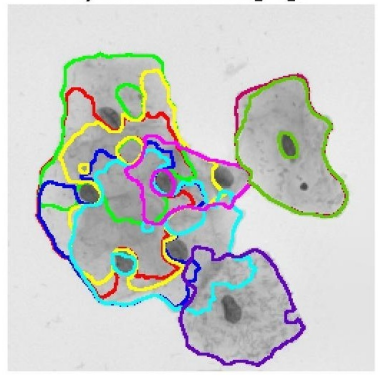

Figure 5.23: Comparing image segmentation results. a) The original image, b) our proposed three stage method, compared to three c)-e) graph-based image segmentation methods [5-7], f) three-step CNN-based image segmentation method [4]. 
a) Original Image

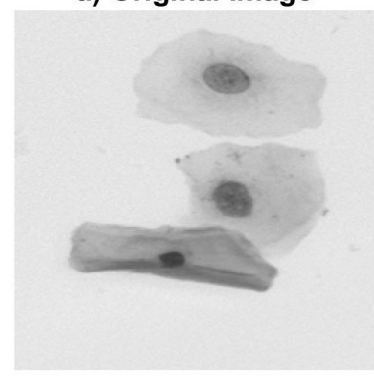

d) Method in [6]

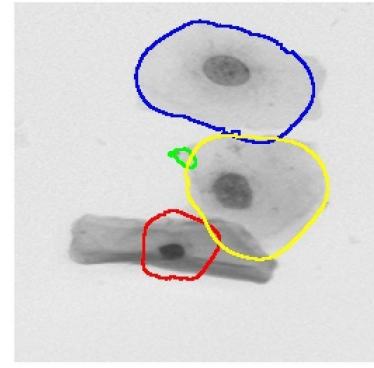

b) Self-learning RW with Seeds

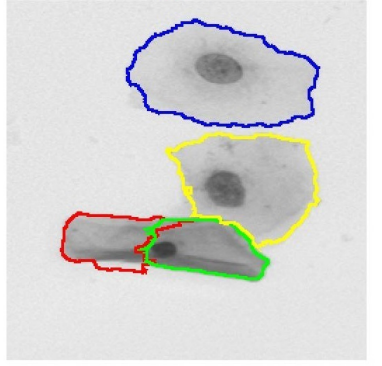

e) Method in [7]

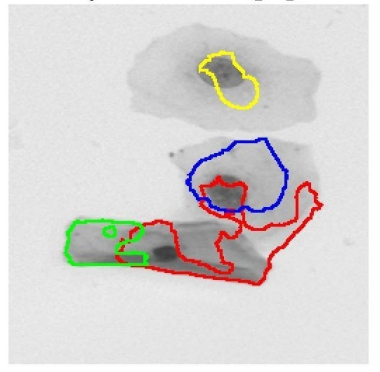

c) Method in [5]

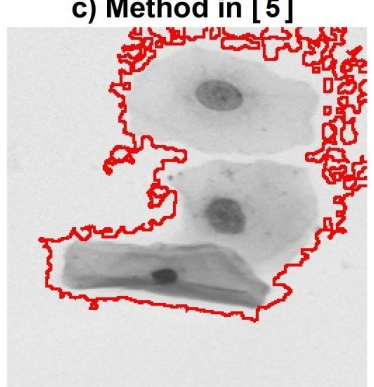

f) Method in [4]

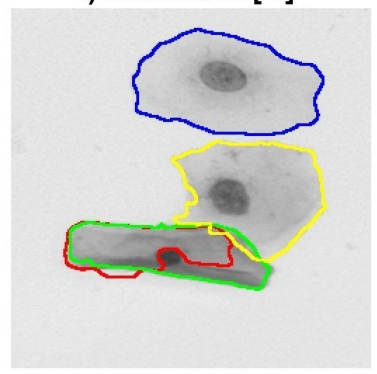

Figure 5.24: Comparing image segmentation results. a) The original image, b) our proposed three stage method, compared to three c)-e) graph-based image segmentation methods [5-7], f) three-step CNN-based image segmentation method [4]. 
a) Original Image



d) Method in [6]

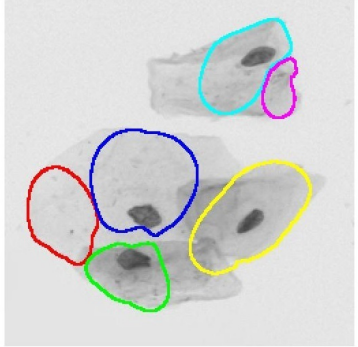

b) Self-learning RW with Seeds

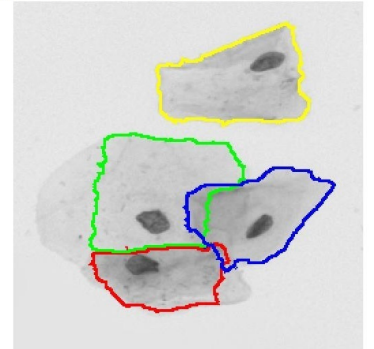

e) Method in [7]

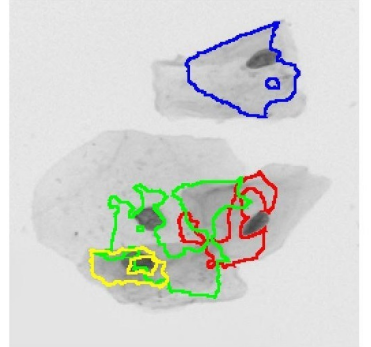

c) Method in [5]

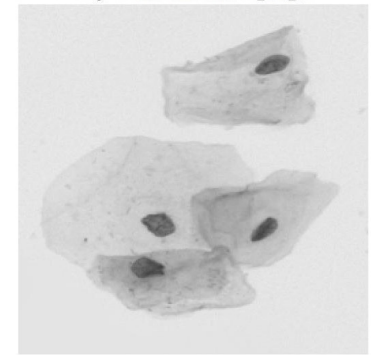

f) Method in [4]

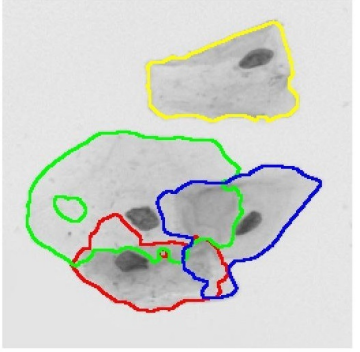

Figure 5.25: Comparing image segmentation results. a) The original image, b) our proposed three stage method, compared to three c)-e) graph-based image segmentation methods [5-7], f) three-step CNN-based image segmentation method [4]. 
a) Original Image

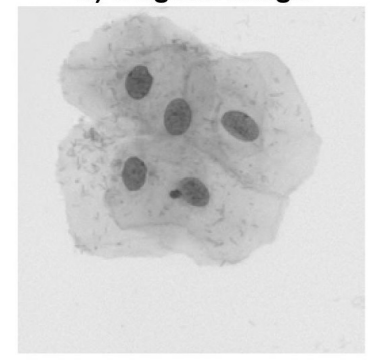

d) Method in [6]

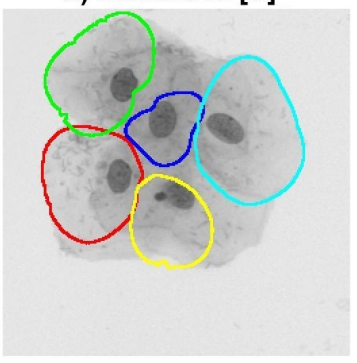

b) Self-learning RW with Seeds

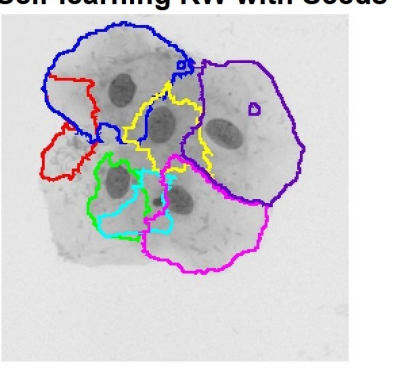

e) Method in [7]

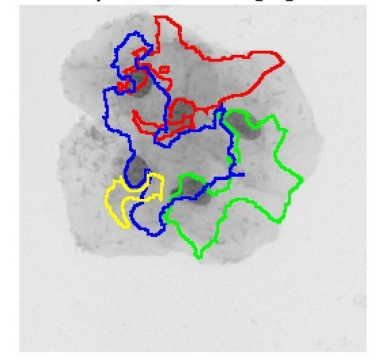

c) Method in [5]

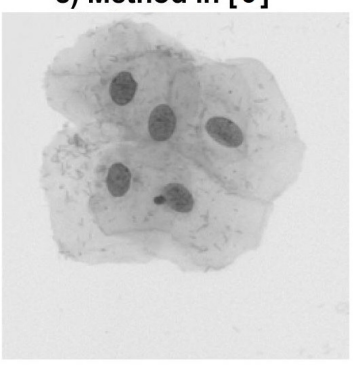

f) Method in [4]

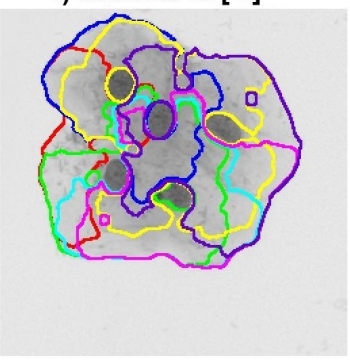

Figure 5.26: Comparing image segmentation results. a) The original image, b) our proposed three stage method, compared to three c)-e) graph-based image segmentation methods [5-7], f) three-step CNN-based image segmentation method [4]. 
Table 5.4: Training times and test times.

\begin{tabular}{|c|c|c|}
\hline Description & Offline training (hours) & Test (sec) \\
\hline Proposed Method & 35.2 & $\mathbf{1 . 1 4}$ \\
\hline Lu et al. [5] & -- & 349.45 \\
\hline Phoulady et al. [6] & -- & 3.53 \\
\hline Sung et al. [7] & 35.2 & 13.74 \\
\hline Wan et al. [4] & 36.4 & 24.72 \\
\hline
\end{tabular}

It is seen in Figures 5.23-5.26 that although none of the methods could find the boundaries of the cells accurately, the proposed method performs better in finding the number of cells and the rough estimate of their boundaries. For example, the method in [5] found wrong approximation of the cells in two of the sample real images shown in Figures 5.23-c and 5.24-c, and was not able to even find any cell segmentation in samples shown in Figures 5.25-c and 5.26-c. The method in [6] found a rough estimation of the segmentation cells, but it could not detect the number of cells accurately (Figures 5.23-d, 5.24-d, 5.25-d, and 5.26-d). The method in [7] fails to find the number of cells and the cytoplasm borders properly (Figures 5.23-e, 5.24-e, 5.25-e, and 5.26-e). Finally, while the method in [4] gives a comparable performance compared to the proposed algorithm in one sample real data (Figure 5.24-f), it does not find the proper number of cells and approximate boundaries for the other three samples (Figures 5.23-e, 5.25-e, 5.26-e). 


\subsection{Summary}

In this chapter, we proposed a CNN-based cervical cell segmentation method. We used our proposed CNN to generate the probabilistic output images for nuclei and cell locations. Having the nuclei output, we applied a multi-layer random walker image segmentation algorithm to find seededbased cell border approximates. Putting a threshold on the probabilistic output for cell borders we also find $\mathrm{CNN}$-based cell border approximates. We then match the CNN-based cell approximates with seeded-based cell approximates using Hungarian algorithm [49]. Finally, we apply the seeded-based region growing or conditional merge for cell border fine tuning. The segmentation masks obtained are then used for cervical cell separation in the next chapter. Although the algorithms give promising results, the methods are tested only on synthetically generated datasets due to the lack of enough labeled real images. Therefore, with the access to a large number of labeled real images, we can train and test our algorithms on real images. 


\section{Chapter 6}

\section{Proposed Two-stage CNN for Image Separation}

In the previous chapter, we proposed a multi-layer random walker image segmentation method for translucent partially overlapped objects. In this chapter, we propose a two-stage method for image separation using translucent partially overlapped objects. In the first stage, we use our proposed residual network for image segmentation from Chapter 5. Then in the second stage, we use the results from the first stage as extra information for image separation.

Our proposed two-stage $\mathrm{CNN}$ is shown in Fig. 6.1 where we show only two cells in the image resulting in two segmented cell images as output for simplicity. Given the challenge of segmenting and separating cell images, an assumption of only two cells is made to simplify the problem in developing our initial proof-of-concept; however, we extend the work for more complex images with 10 jointly overlapping cells.

\subsection{Proposed CNN}

In the previous chapter we proposed CNNs for image segmentation. We compared the proposed networks for accuracy and time complexity. In this chapter, we choose the residual network for image segmentation and we design a new residual network for image separation. 
First Stage

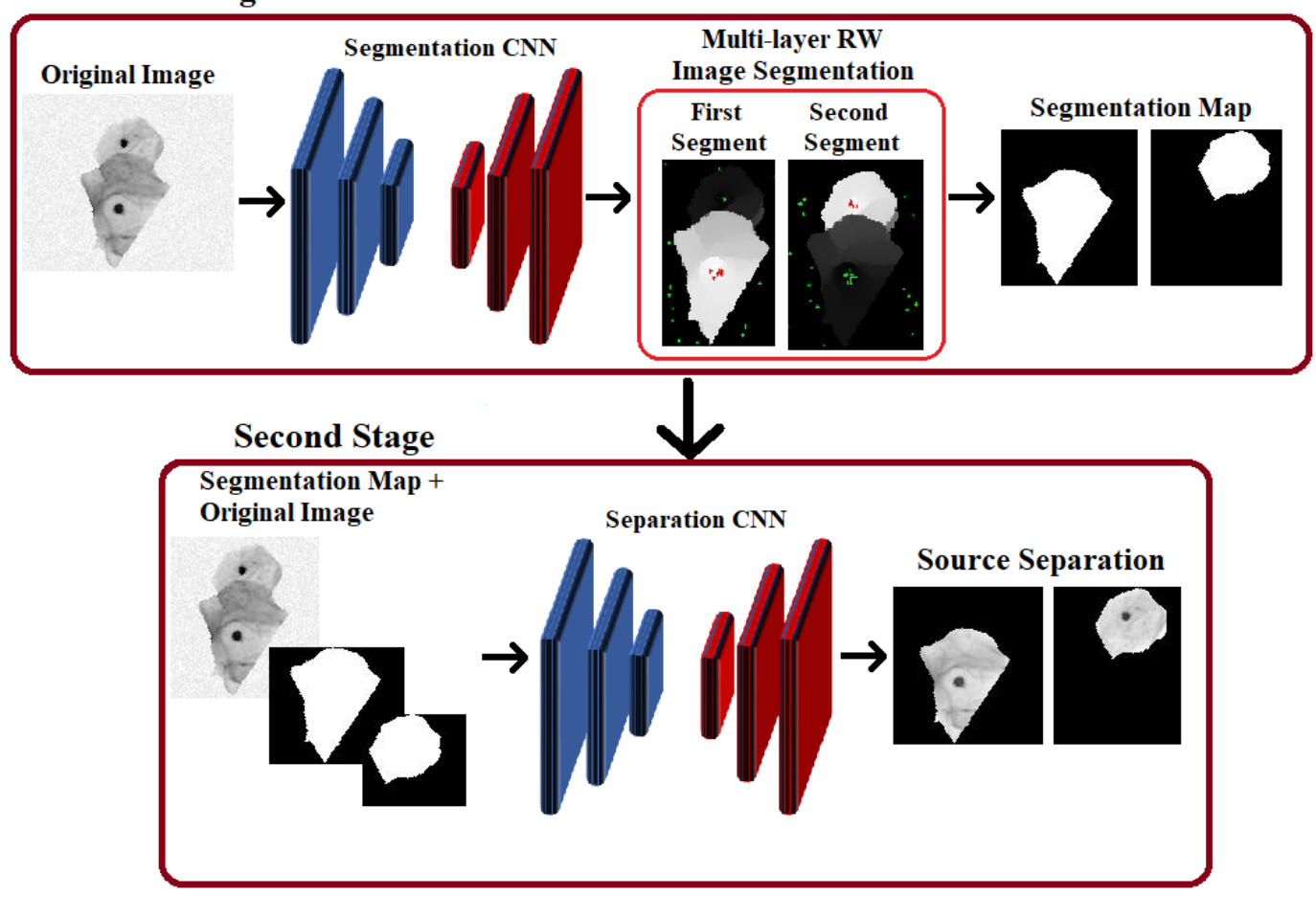

Figure 6.1: Proposed two-stage CNN. 


\subsubsection{Two-Stage CNN for Image Separation}

The first stage of the proposed method uses our work in Chapter 5 applied to segment translucent partially-overlapped cervical cells in cytology images [44]. The network structure in this first stage is shown in Figure 5.3 where the cytology image with overlapping cervical cells is fed in as input and the output is multiple images, each containing the segmentation of one of the cells.

Following cell segmentation, the second step of the proposed method performs the actual cell image separation. For the cell separation, a residual CNN is designed with 66 layers consisting of an input layer (1 layer), an encoder network (31 layers), a decoder network (26 layers), a Dense layer with linear activation (1 layer), an output layer (1 layer), and three sets of skip connection layers, each having 2 layers, a convolution layer and normalization layer (6 layers). The structure of the residual $\mathrm{CNN}$ is shown in Fig. 6.2. In the proposed CNN for image separation, the input is the original cytology image along with the image segmentation masks for each cell as determined from the first stage of our proposed method. The network will be run multiple times, each time produces one of the individual cervical cells.

Because of the under-determined nature of the single-image separation problem, it is essential to use non-linearity in the network. Dense layers in CNNs add interesting non-linearity properties and, therefore, they can model any mathematical functions. But, the computational complexity of dense layers is higher than convolutional layers. So, we use dense layers at the deepest layers of the network with smallest image sizes. On the other hand, because the output of our network performs regression, it should be linear. Therefore, our network should have a linear activation layer at the end. So, we use a dense layer with a linear activation layer at the end. Also the designed CNN in Fig. 6.2 uses residual connections to allow a deeper network that will still converge when trained [34]. The details of the layers for our proposed image separation CNN is shown in Appendix A.3. 


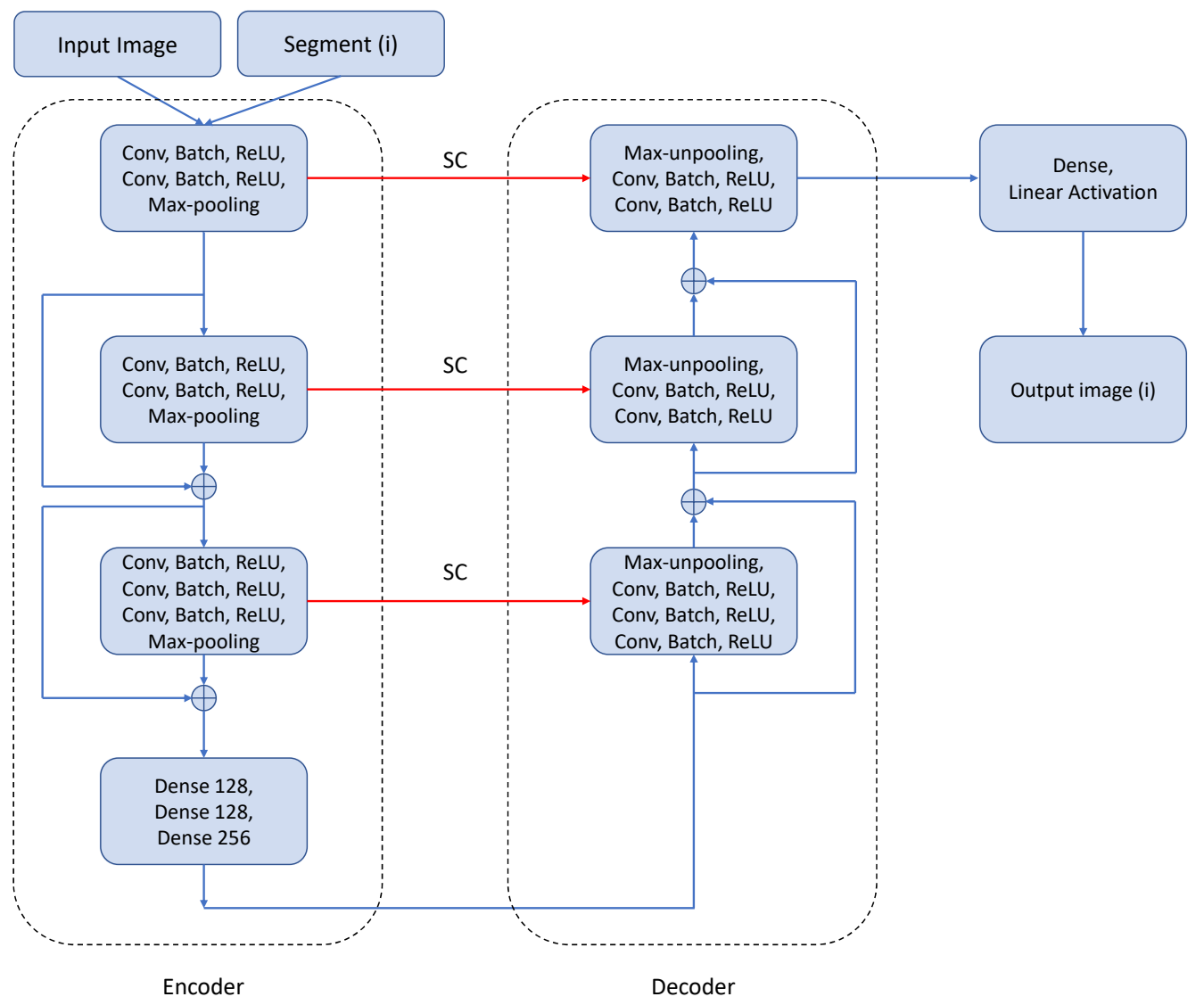

Figure 6.2: Proposed residual network for image separation. 


\subsection{Data Setup}

Since training a CNN works best with a large amount of data, we created images from individual real cervical cells images that we synthetically manipulated, overlapped, and combined. We created two different datasets, one with only two cells partially overlapped and a second one with 10 different cells jointly overlapped.

To evaluate our method, we calculated the segmentation accuracy using (3.24), (3.25) for the first phase. For image separation performance, we measured peak signal-to-noise ratio, PSNR, (6.1) and structural similarity measure (SSIM) [104]

$$
P S N R=10 \log _{10} \frac{255^{2}}{M S E} d B
$$

where $M S E$ is the mean square error

$$
M S E=\frac{1}{m n} \sum_{i=0}^{m-1} \sum_{j=0}^{n-1}[G(i, j)-R(i, j)]^{2}
$$

and where $m \times n$ is the total number of image pixels and $G, R$ are the original source and image separation results, respectively.

\subsubsection{Two Overlapping Cells}

We extracted 24 individual cervical cell images from the ISBI 2014 Overlapping Cervical Cytology Image Segmentation Challenge dataset [15]. With these cell images, we applied random affine transforms to increase variation in our cell sample population. Then, we created synthetic images by overlapping and combining two cell images using the Beer-Lambert law [14].

Following the above approach, we generated 50000 synthetic images by overlapping and combining real cervical cell images using (5.2). In (5.2), we averaged over the background area for all the training images in the ISBI 2014 dataset [15] to find $E_{0}$ and used $\alpha_{1}=0.95$ and $\alpha_{2}=0.9$. Fig. 6.3 shows a few synthetic image mixtures we created using (5.2). 

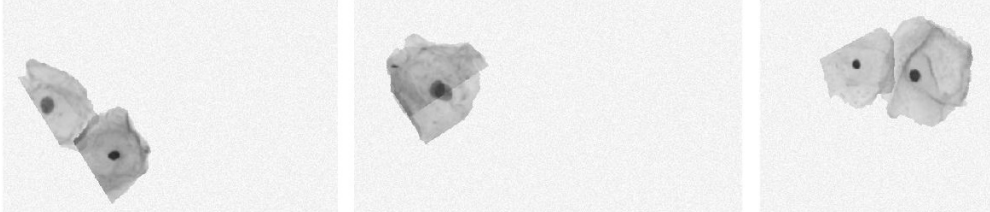

Figure 6.3: A few synthetic image mixtures of partially overlapped two cervical cells using BeerLambert law (5.2).

\subsubsection{Results}

To evaluate our proposed network, we trained a CNN using Python Keras package with 50 epochs using 49000 training data with image size $256 \times 256$. We used a learning rate of 0.001 and Adam optimizer [100] with first and second momentum parameters $\beta_{1}=0.9$ and $\beta_{2}=0.999$. Figures 6.4 and 6.5 show the segmentation and separation results for the proposed network, respectively.

Because there is no work done for cervical cell separation, we were not able to compare our proposed cervical cell separation method with other methods. However, for the segmentation part we compared our proposed segmentation method with two top segmentation methods for overlapping cervical cells $[5,6]$. Table 6.1 shows the accuracy of the segmentation results calculated by (3.24), (3.25). The results are averaged over 1000 test images.

In Table 6.1, mean and std represent the average and standard deviation values for the measures. For simplicity to compare different methods, the best value for each segmentation quantitative measurement is represented as the bold font. It is seen that our method outperforms the two top methods on our dataset. Table 6.2 shows the PSNR and SSIM values for the separation results. The results are averaged over 1000 test images.

In Table 6.2 mean and std represent the average and standard deviation values for the measures. Although our proposed image separation method works for most of the cases in our dataset, it 
b) Ground Truth Image Segmentation

a) Original Image

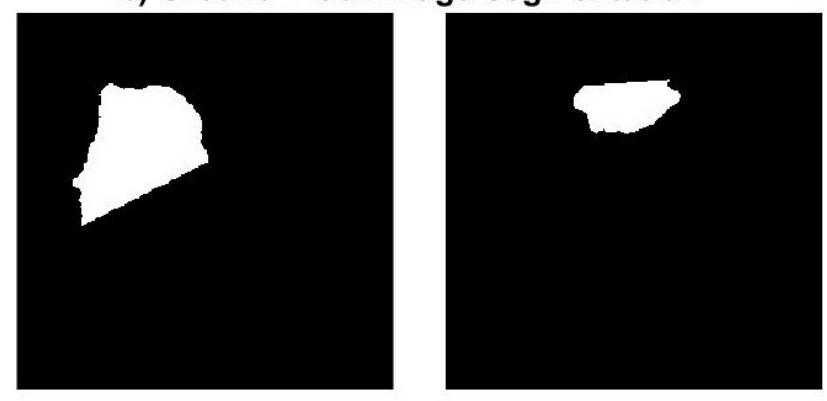

c) Image Segmentation Results (First Phase), Acc: 99.8566 , loU: 97.4529
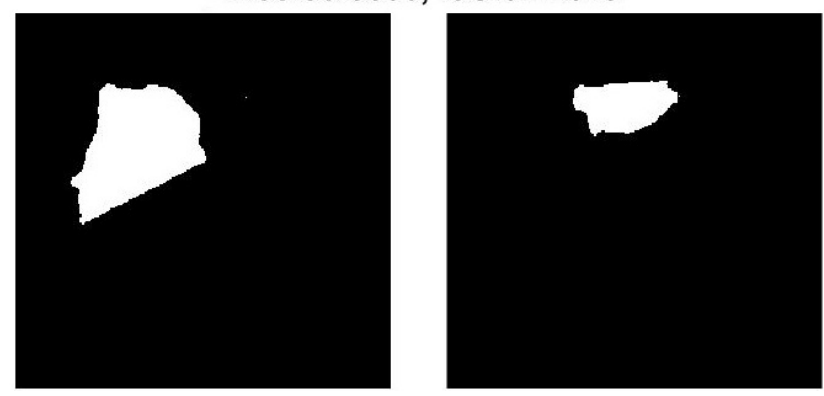

Figure 6.4: Results for the segmentation CNN. a) Original image, b) ground truth segmentation, c) segmentation results. 
b) Ground Truth Image Sources

a) Original Image
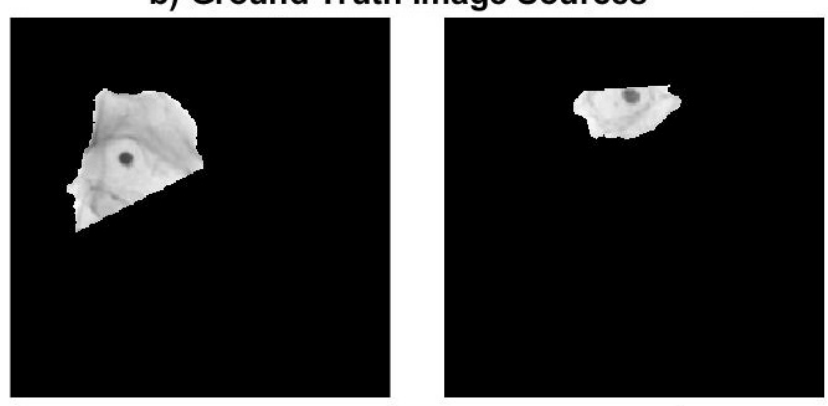

c) Image Separation Result (Second Phase), PSNR:27.52 dB, SSIM:0.98
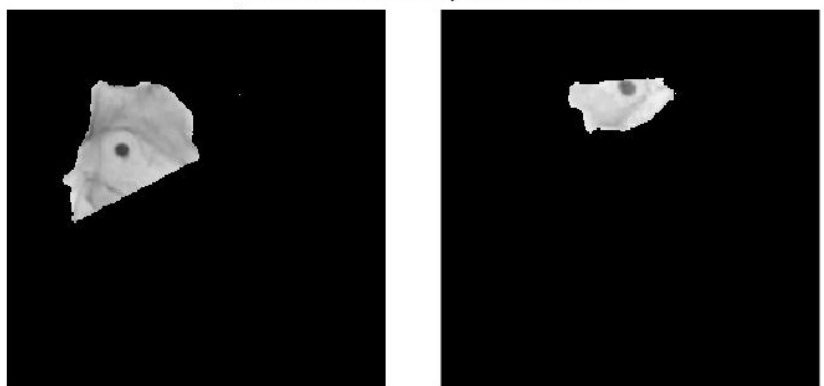

Figure 6.5: Results for the separation CNN. a) Original image, b) ground truth original sources, c) separation results. The PSNR and SSIM values are averaged over the two decomposed images. 
Table 6.1: Segmentation quantitative measure.

\begin{tabular}{|c|c|c|c|c|}
\hline \multirow{2}{*}{ Description } & \multicolumn{2}{|c|}{ Acc (\%) } & \multicolumn{2}{c|}{ IoU (\%) } \\
& mean & std & mean & std \\
\hline Proposed Segmentation & $\mathbf{9 9 . 8 9}$ & $\mathbf{0 . 0 6}$ & $\mathbf{9 7 . 4 2}$ & $\mathbf{1 . 3 8}$ \\
\hline Lu et al. [5] & 99.18 & 1.26 & 84.20 & 18.54 \\
\hline Phoulady et al. [6] & 99.09 & 0.65 & 80.93 & 13.32 \\
\hline
\end{tabular}

fails in some cases. Because our method has two stages, the results for the image separation stage depend on image segmentation results from the first stage. Therefore, poor image segmentation results lead to poor image separation results. To evaluate the second phase of our proposed method, we calculated PSNR and SSIM when the ground truth image segmentation is used as the first phase. The results are shown in Table 6.2 second row.

Figures 6.6 and 6.7 compare the results of image separation when the image segmentation is good and poor, respectively.

In Figures 6.6 and 6.7, sub-images a) and b) show the ground truth image segmentation masks and image sources respectively. Results for first phase and second phase of our proposed method are shown in sub-images c) and d). We used the results from sub-image d) and regenerated the image mixture using (5.2). The difference between real original image and the regenerated image is shown in sub-images g) and h) in linear and dB scales respectively. In Figures 6.6-g, 6.6-h, 6.7$\mathrm{g}$, and 6.7-h, black areas represent zero intensity value. It can be seen that in 6.6-g, the difference between the regenerated image and original image is more around the boundaries of the cells and the rest of the images are very similar. Therefore, the SSIM measure gives high value for image 
a) Ground Truth Image Segmentation

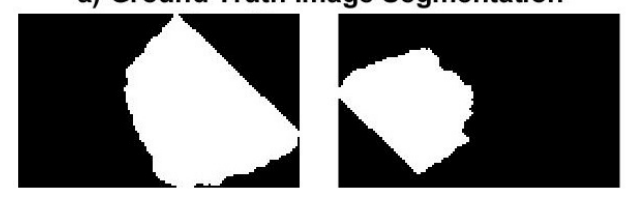

c) Image Segmentation Results (First Phase), Acc:99.95, loU:98.69

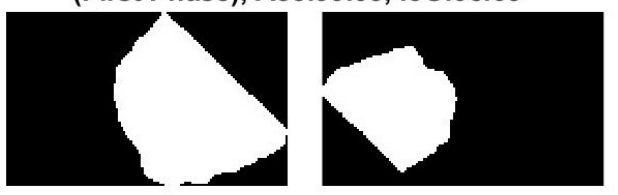

e) Original Image

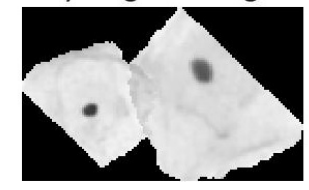

f) Regenerated Image

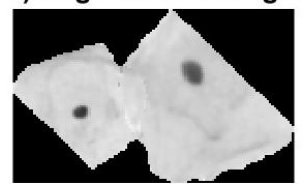

b) Ground Truth Image Sources

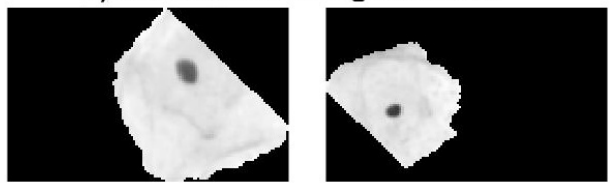

d) Image Separation Results (Second Phase), PSNR:31.32 dB, SSIM:1.00

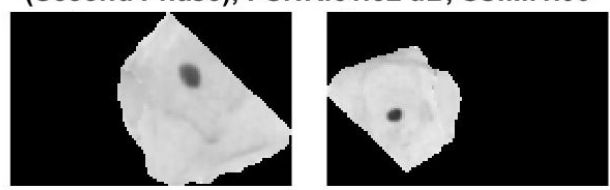

h) Residual Image

g) Residual Image

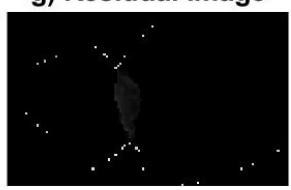
dB Scale

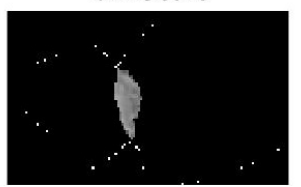

Figure 6.6: Good image segmentation leads to good image separation and small residual image.

a) Ground truth image segmentation, b) ground truth image sources, c) image segmentation results, d) image separation results, e) original image, f) regenerated image, g) and h) residual image in linear and $\mathrm{dB}$ scales respectively. 


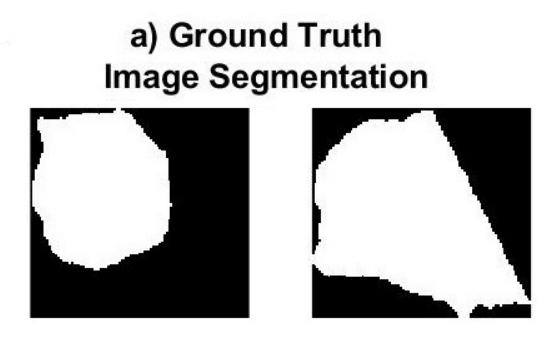

c) Image Segmentation Results (First Phase), Acc:99.47, loU:92.48


e) Original Image

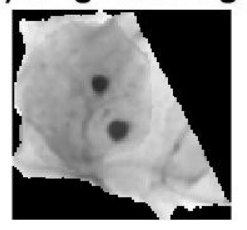

f) Regenerated Image

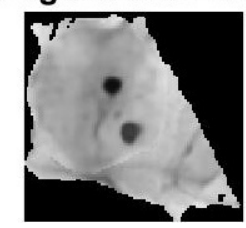

b) Ground Truth Image Sources
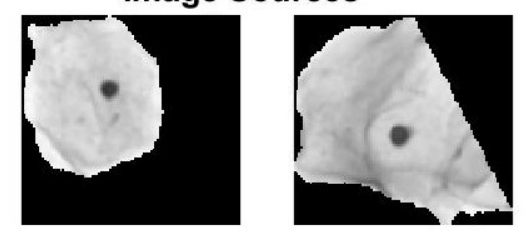

d) Image Separation Results

(Second Phase), PSNR:21.98 dB, SSIM:0.94
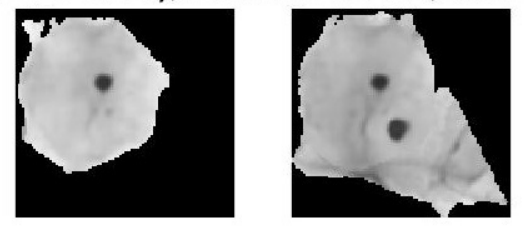

h) Residual Image

g) Residual Image

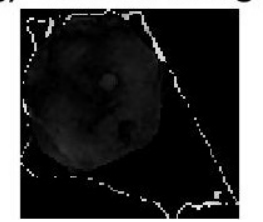

dB Scale

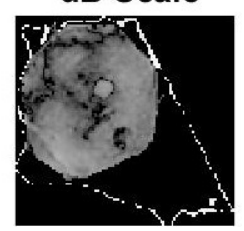

Figure 6.7: Poor image separation and large residual image as a result of poor image segmentation.

a) Ground truth image segmentation, b) ground truth image sources, c) image segmentation results, d) image separation results, e) original image, f) regenerated image, g) and h) residual image in linear and $\mathrm{dB}$ scales respectively. 
Table 6.2: Separation quantitative measure.

\begin{tabular}{|c|c|c|c|c|c|}
\hline \multirow{2}{*}{ Description } & \multicolumn{2}{|c|}{ PSNR (dB) } & \multicolumn{2}{|c|}{ SSIM } \\
& mean & std & mean & std \\
\hline Proposed Separation Using First Stage Segmentation Results & 28.78 & 1.73 & 0.9869 & 0.0052 \\
\hline Proposed Separation Using Ground Truth Segmentation & 45.29 & 4.78 & 0.9990 & 0.0024 \\
\hline
\end{tabular}

separation. However, the difference of intensity values in the overlap area is small but not zero (Figure 6.6-g and 6.6-h). Also, the difference of intensity values around the boundaries is high; therefore, PSNR is not giving a high value.

\subsubsection{Jointly Overlapped Multiple Cervical Cells}

In our previous experiment we used an image mixture with two overlapping cervical cells. However, in real cervical cell images, there can be more than two partially overlapping cervical cells. In order to extend our work, we prepare a dataset of images with more cervical cells. Since training a $\mathrm{CNN}$ works best with a large amount of data, we created images from individual real cervical cells images that we synthetically manipulated, overlapped, and combined. We extracted 65 individual cervical cell images from the ISBI 2014 Overlapping Cervical Cytology Image Segmentation Challenge dataset [15]. With these cell images, we applied random affine transforms to increase variation in our cell sample population. Then, we created synthetic images by jointly overlapping and combining cell images using Algorithm 5.

For the first step of our method, we used our Jointly Overlapped Cervical Cell Segmentation method from Chapter 5 to extract the segmentation map of the cells. For the second step, we 

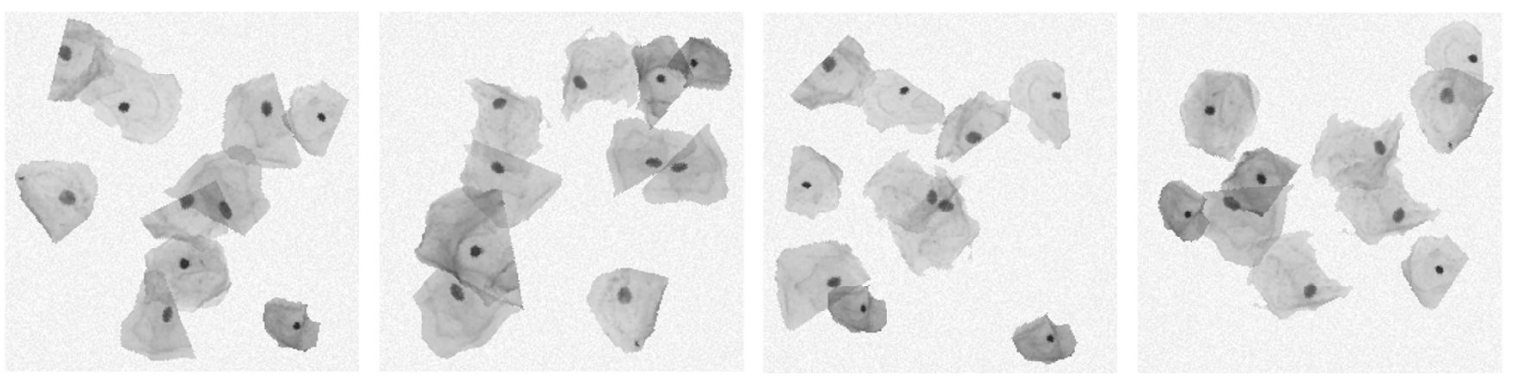

Figure 6.8: A few synthetic image mixtures of jointly overlapped cervical cells using Beer-Lambert law (5.2).

trained a CNN using Python Keras package with 50 epochs using 35000 training data with image size $256 \times 256$. We used a learning rate of 0.001 and Adam optimizer [100] with first and second momentum parameters $\beta_{1}=0.9$ and $\beta_{2}=0.999$. The input to the network is the image mixture along with the ground truth segmentation map of one of the cervical cells and the output of the network is the image intensity values of that cervical cell. Fig. 6.8 shows a few synthetic image mixtures using (5.2).

\subsubsection{Results}

We used the results of the image segmentation from the first stage for image separation in the second stage. Figures 6.9 and 6.10 show the separation results for the proposed two-stage method. For simplicity, we show all 10 cells in two non-overlapped images.

In Figure 6.10, areas with zero intensity value are shown in black. It is seen that the residual image has high intensity values around the borders of the cells, therefore, the PSNR measure gives a low value.

Table 6.3 shows the PSNR and SSIM values for the separation results. The results are averaged over 100 test images, where mean and std represent the average and standard deviation values of the measures. Similar to the previous section, the results for the image separation stage depend 
b) Ground Truth Image Segmentation

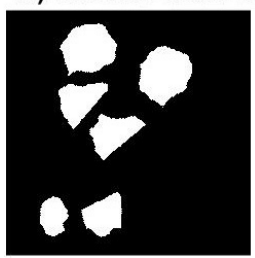

d) Image Segmentation Results (First Phase), Acc:99.8659, loU:95.1194

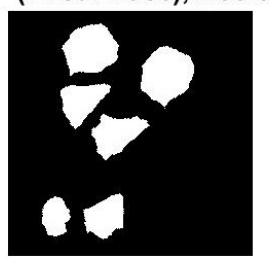

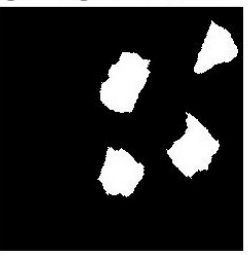

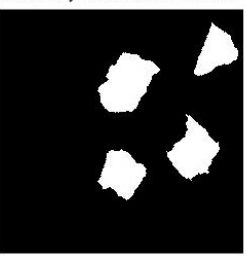

c) Ground Truth Image Sources
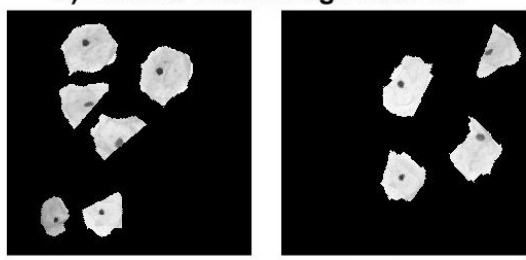

e) Image Separation Results (Second Phase), PSNR: $26.69 \mathrm{~dB}$, SSIM: 0.97
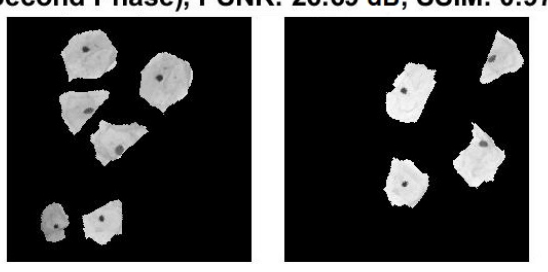

Figure 6.9: Results for our proposed two-stage method. a) Original image, b) ground truth image segmentation, c) ground truth image sources, d) image segmentation results, e) image separation results. The Acc, IoU, PSNR, and SSIM values are averaged over the 10 separated cells in the image mixture. Cells are shown in two non-overlapped images for simplicity. 
a) Ground Truth

Image Segmentation
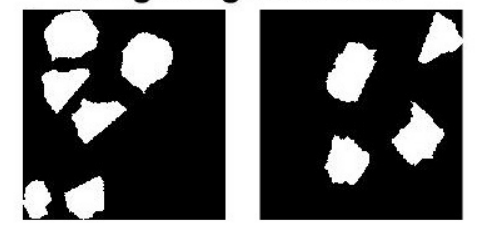

c) Image Segmentation Results

(First Phase), Acc:99.87, loU:95.12
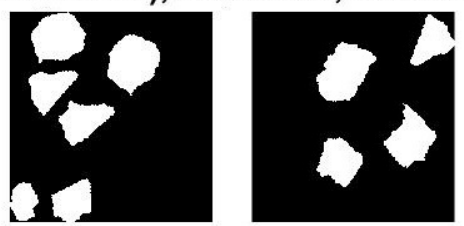

e) Original Image

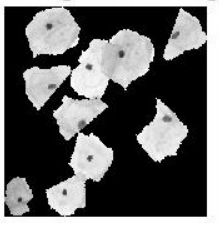

f) Regenerated Image

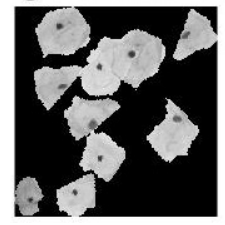

b) Ground Truth Image Sources

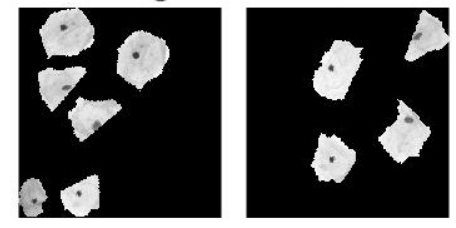

d) Image Separation Results

(Second Phase), PSNR:20.56 dB, SSIM:0.97
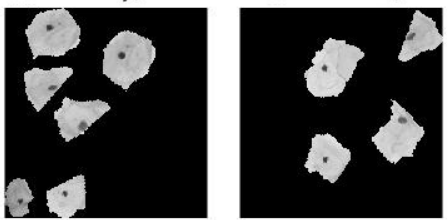

g) Residual Image

h) Residual Image



dB Scale

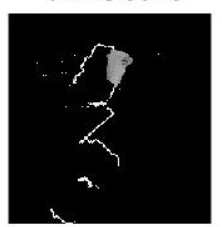

Figure 6.10: Results for our proposed two-stage method. a) Ground truth image segmentation, b) ground truth image sources, c) image segmentation results, d) image separation results, e) original image, f) regenerated image, g) and h) residual image in linear and $\mathrm{dB}$ scales respectively. The Acc, IoU, PSNR, and SSIM values are averaged over the 10 separated cells in the image mixture. Cells are shown in two non-overlapped images for simplicity. 
Table 6.3: Separation accuracy.

\begin{tabular}{|c|c|c|c|c|c|}
\hline & \multicolumn{2}{|c|}{ PSNR (dB) } & \multicolumn{2}{|c|}{ SSIM } \\
\hline mescription & mean & std & mean & std \\
\hline Proposed Separation Using First Stage Segmentation Results & 17.25 & 4.47 & 0.9375 & 0.0445 \\
\hline Proposed Separation Using Segmentation Method in [5] & 15.80 & 2.07 & 0.9139 & 0.0212 \\
\hline Proposed Separation Using Segmentation Method in [6] & 15.13 & 2.18 & 0.9094 & 0.0241 \\
\hline Proposed Separation Using Segmentation Method in [7] & 15.12 & 2.19 & 0.9094 & 0.0242 \\
\hline Proposed Separation Using Segmentation Method in [4] & 15.83 & 2.09 & 0.9142 & 0.0215 \\
\hline Proposed Separation Using Ground Truth Segmentation & 37.79 & 2.39 & 0.9996 & 0.0001 \\
\hline
\end{tabular}

on image segmentation results from the first stage. In order to evaluate the second phase of our proposed method independently, we use ground truth image segmentation for the first stage. To be able to compare our method with others, we used the segmentation results from our proposed method and competing methods for our separation step. We also used the ground truth image segmentation to evaluate only the separation step.

\subsection{Summary}

In this chapter, we implemented a CNN for cervical cell separation. Similar to our proposed segmentation $\mathrm{CNN}$, our separation $\mathrm{CNN}$ includes an encoder and a decoder. We used dense layers in the deepest layers of our network to add non-linearity to our system while keeping the network as 
less complex as possible. A dense layer with linear activation produces the separation output. The proposed CNN takes the segmentation map of one cell along with the original cervical cell image and produces the intensity values for that cell. For images with $N$ number of cells, the CNN will be run $N$ times. To isolate the performance of separation $\mathrm{CNN}$ from the segmentation $\mathrm{CNN}$ one can bypassed the first step by using the ground truth segmentation masks of cells. However, for real images with no segmentation ground truth, one should use the two-step method to provide the cell separation. 


\section{Chapter 7}

\section{Conclusion and Future Work}

\subsection{Conclusion}

In this dissertation, we design a two-stage $\mathrm{CNN}$ for image decomposition. We focus on overlapped cervical cell images and use image segmentation information for under-determined image decomposition. We first propose some novel methods to segment partially overlapped translucent objects. We then use these image segmentation masks for image decomposition.

Starting with weakly supervised seed-based image segmentation, we present a novel graphbased method that creates a multi-layer graph from a $2 D$ image. To diffuse the image values within the graph-layers and not between them, we generate a block diagonal Laplacian matrix that has the $2 D$ Laplacian matrix on the diagonals to separate the graph layers. We then add a few seeds provided by the user. The algorithm then finds regions in a multi-layer output image, each region in a separate output layer. Our results on the synthetic images show the strength of our proposed method.

Because providing a lot of seeds might sometimes be time consuming for the user this method is not suitable for the real-time segmentation applications. Therefore, we extend our work to remove the need for the user-defined seeds. We present a novel supervised image segmentation method 
that uses data priors instead of seeds [45]. In the training phase, for each selected pixel on nonoverlapped regions, we create a $5 \times 5$ window centered at that pixel and generate a $25 \mathrm{D}$ vector of image intensity values. Then we use Euclidean distance at this $25 D$ vector to create the feature space. To create data-term we define the similarity measure as the distances between the seeds' feature vectors and the nodes' feature vectors in the image. We finally use the method in [3] to generate a multi-layer Laplacian matrix. Our method first generates data-term for each segment in the training phase. A new energy function consisting of the generated data-term and the multilayer Laplacian matrix is then obtained and solved to find the regions in the image, each region in a separate output layer. Our results on the synthetic images show the strength of our proposed method compared to [1-3].

However, finding the proper feature space depends on the application and varies by changing the data type. Convolutional neural networks (CNNs) have the advantage of finding important features automatically. Researchers have shown interest in CNNs specifically in computer vision applications and recently CNNs are widely used for image segmentation problems.

We use CNN approaches to segment translucent partially overlapped objects [44]. Although $\mathrm{CNN}$ is good at generalizing the training, it is not very good at getting highly accurate edges at the current implementations for cervical cell segmentation $[4,43]$. Therefore, $\mathrm{CNN}$ is good to give a rough segmentation of the cells, and then we have to do edge refinement after.

Random walker image segmentation method is good at region growing so it can be used as the refinement step to fine tune the $\mathrm{CNN}$ segmentation results. However, random walker struggles when it has poor initial setup, so if the seeds are not extracted correctly, the final segmentation results will be poor.

By using a combination of $\mathrm{CNN}$ and random walker image segmentation, we take the advantage of each part and remove the disadvantage of it. Different from other methods that use discrete segmentation results from $\mathrm{CNN}[4,43]$ for fine tuning, we use CNN probabilistic map to try to 
guide the random walker at the refinement step.

Since training a CNN works best with a large amount of data, we create images from individual real cervical cells images that we synthetically manipulated, overlapped, and combined. We extracted 573 individual cervical cell images from the ISBI 2014 Overlapping Cervical Cytology Image Segmentation Challenge dataset [15]. With these cell images, we apply random affine transforms to increase variation in our cell sample population. Then, we create synthetic images by overlapping and combining multiple cell images using the Beer-Lambert law [14]. To evaluate our method we compare our three step segmentation algorithm with three known methods for overlapped cervical cell segmentation methods using our synthetically manipulated dataset.

We finally design a two-stage CNN for image decomposition. We use deep learning for overlapped cervical cell image separation [47]. Although deep learning is widely used for other image applications, we believe that there is no work done yet for cervical cell separation. We implement a two-stage CNN for image decomposition. In the first stage, the network segments the partially overlapped translucent regions and this segmentation information is then fed into the second stage of the network for image separation. To evaluate our method we create a dataset of 50000 training images with two partially overlapped cervical cells and a dataset of 35000 training images with 10 jointly overlapped cervical cells. Results for the first dataset show segmentation accuracy of greater than $97 \%$ and decomposition SSIM score of more than 0.99 . For the second dataset we use our three step segmentation algorithm first to create the segmentation map of the image mixture. We then used the results of image segmentation step for image decomposition. Decomposition results show segmentation accuracy of greater than $99 \%$ and decomposition SSIM score of more than 0.93 . 


\subsection{Future Work}

The proposed segmentation-based separation method provides promising results on synthetically generated data. However, because the separation proposed model consists of two stages, poor results for the image segmentation stage lead to poor image decomposition results. Also, the permutation problem that commonly effects signal separation occasionally occurs resulting in some cell structure mis-separation (for example, one cell given two nuclei and the other given none). Separation results show an increased improvement when using ground truth segmentation instead of the image segmentation results from the first stage. Using a joint segmentation-separation CNN will improve the separation and segmentation results. therefore, one way to improve the final image separation results is to use a combined network that trains the segmentation and separation at the same time.

Also, one of the draw backs of our CNN implementation for both image segmentation and image decomposition networks is that we used max-pool layers at the end of each set of layers to decrease the complexity of the network. In other words our segmentation and decomposition CNNs are UNet-based shape so they decrease the image size after each set of layers and therefore, lose the image information at the deeper layers of the network. So, another way to improve the results is either to use larger image size for training or to slowly decrease the image size in the intermediate network layers.

Although our proposed CNNs for image segmentation and image separation show promising results for our synthetically generated datasets, One might get advantage by using pre-trained networks. Also they are not the only way to implement segmentation and separation CNNs. One way to extend this work is to explore different CNNs, or implement the different CNNs for better performance.

Also, in Chapter 5, many thresholds are used were the values are empirically obtained. However, one can train a small CNN to optimize finding these threshold values. 
Finally, the algorithms in this dissertation were evaluated on synthetically generated datasets. One way to extend the work is to apply the algorithms on images with more overlapped regions, or apply it on real images considering to have enough labeled real images. 


\section{List of References}

[1] L. Grady, "Random walks for image segmentation," IEEE Transactions on Pattern Analysis and Machine Intelligence, vol. 28, pp. 1-17, Nov 2006.

[2] L. Grady, "Multilabel random walker image segmentation using prior models," in CVPR, pp. 763-770, 2005.

[3] T. L. Mahyari and R. M. Dansereau, "Random walks for image segmentation containing translucent overlapped objects," in 2017 IEEE Global Conference on Signal and Information Processing (GlobalSIP), pp. 46-50, Nov 2017.

[4] "Accurate segmentation of overlapping cells in cervical cytology with deep convolutional neural networks," Neurocomputing, vol. 365, pp. 157-170, 2019.

[5] Z. Lu, G. Carneiro, and A. Bradley, "An improved joint optimization of multiple level set functions for the segmentation of overlapping cervical cells," IEEE Transactions on Image Processing, vol. 24, Jan 2015.

[6] H. A. Phoulady, D. B. Goldgof, L. O. Hall, and P. R. Mouton, "A framework for nucleus and overlapping cytoplasm segmentation in cervical cytology extended depth of field and volume images," Computerized Medical Imaging and Graphics, vol. 59, pp. 38-49, 2017.

[7] M. Sung and L. Chang, "Using multi-layer random walker for image segmentation," in 2018 International Workshop on Advanced Image Technology (IWAIT), pp. 1-4, 2018.

[8] A. Schwaninger, "Increasing efficiency in airport security screening," WIT Transactions on the Built Environment, vol. 82, pp. 407-416, 012005.

[9] Papanicolaou (PAP) Red Stain Kit (Cytology Stain), available on 30 Apr 2021. https://www.abcam.com/ papanicolaou-pap-red-stain-kit-cytology-stain-ab150679.html. 
[10] C. V. Bastian, A. Schwaninger, and S. Michel, "Do multi-view x-ray systems improve xray image interpretation in airport security screening?," Zeitschrift für Arbeitswissenschaft, vol. 62, pp. 165-173, 012008.

[11] D. S. Heller, OB-GYN pathology for the clinician: A practical review with clinical correlations. Springer International Publishing, first ed., Jan 2015.

[12] V. Badrinarayanan, A. Kendall, and R. Cipolla, "Segnet: A deep convolutional encoderdecoder architecture for image segmentation," IEEE Transactions on Pattern Analysis and Machine Intelligence, vol. 39, no. 12, pp. 2481-2495, 2017.

[13] USC texture database, available on 30 Apr 2021. http://sipi.usc.edu/ database/database.php?volume=textures.

[14] J. Zhang, Z. Hu, G. Han, and X. He, "Segmentation of overlapping cells in cervical smears based on spatial relationship and overlapping translucency light transmission model," Pattern Recognition, vol. 60, 052016.

[15] Overlapping Cervical Cytology Image Segmentation Challenge - ISBI 2014, available on 30 Apr 2021. https://cs.adelaide.edu.au/ carneiro/isbil4_ challenge/.

[16] S. Ruder, "An overview of gradient descent optimization algorithms," CoRR, vol. abs/1609.04747, Jun 2017.

[17] Y. NESTEROV, "A method for unconstrained convex minimization problem with the rate of convergence $o\left(1 / k^{2}\right)$, , Doklady AN USSR, vol. 269, pp. 543-547, 1983.

[18] B. J. Kim, "Improved deep learning algorithm," Journal of advanced information technology and convergence, vol. 8, no. 2, pp. 119-127, 2018.

[19] RMSprop optimization method, available on 30 Apr 2021. https://engmrk.com/ rmsprop/.

[20] Four different SGD methods, available on 30 Apr 2021. https://tiddler.github. io/optimizers/.

[21] W. J. Song, S. G. Choi, and E. S. Lee, "Prediction and comparison of electrochemical machining on shape memory alloy(sma) using deep neural network(dnn)," Journal of Electrochemical Science and Technology, vol. 10, no. 3, pp. 276-283, 2019. 
[22] L. Miao and H. Qi, "Endmember extraction from highly mixed data using minimum volume constrained nonnegative matrix factorization," IEEE Transactions on Geoscience and Remote Sensing, vol. 45, no. 3, pp. 765-777, 2007.

[23] Y. X. Wang and Y. J. Zhang, "Nonnegative matrix factorization: A comprehensive review," IEEE Transactions on Knowledge and Data Engineering, vol. 25, pp. 1336-1353, Jun 2013.

[24] M. Jena, S. P. Smita, and D. Mishra, "A survey on applications of machine learning techniques for medical image segmentation," International Journal of Engineering and Technology, vol. 7, pp. 4489-4495, Jan 2018.

[25] H. Li, Y. Li, and F. Porikli, "Deeptrack: Learning discriminative feature representations online for robust visual tracking," IEEE Transactions on Image Processing, vol. 25, no. 4, pp. 1834-1848, 2016.

[26] F. Xing, Y. Xie, H. Su, F. Liu, and L. Yang, "Deep learning in microscopy image analysis: A survey," IEEE Transactions on Neural Networks and Learning Systems, vol. 29, no. 10, pp. 4550-4568, 2018.

[27] J. Jotheeswaran and S. Tanwar, "Survey on deep learning for medical imaging," JASC:Journal of Applied Science and Computations, vol. 5, pp. 1608-1620, Dec 2018.

[28] J. Schmidhuber, "Deep learning in neural networks: An overview," Neural Networks, vol. 61, pp. 85-117, Jan 2015.

[29] R. Haridas and J. R. L, "Convolutional neural networks: A comprehensive survey," International Journal of Applied Engineering Research, vol. 14, no. 3, pp. 780-789, 2019.

[30] A. Krizhevsky, I. Sutskever, and G. E. Hinton, "Imagenet classification with deep convolutional neural networks," in Advances in Neural Information Processing Systems 25 (F. Pereira, C. J. C. Burges, L. Bottou, and K. Q. Weinberger, eds.), pp. 1097-1105, Curran Associates, Inc., 2012.

[31] K. Simonyan and A. Zisserman, "Very deep convolutional networks for large-scale image recognition," CoRR, vol. abs/1409.1556, 2014.

[32] C. Szegedy, Wei Liu, Yangqing Jia, P. Sermanet, S. Reed, D. Anguelov, D. Erhan, V. Vanhoucke, and A. Rabinovich, "Going deeper with convolutions," in 2015 IEEE Conference on Computer Vision and Pattern Recognition (CVPR), pp. 1-9, 2015. 
[33] M. D. Zeiler and R. Fergus, "Visualizing and understanding convolutional networks," in Computer Vision - ECCV 2014 (D. Fleet, T. Pajdla, B. Schiele, and T. Tuytelaars, eds.), pp. 818-833, Springer International Publishing, 2014.

[34] K. He, X. Zhang, S. Ren, and J. Sun, “Deep residual learning for image recognition,” CoRR, vol. abs/1512.03385, 2015.

[35] S. Pouyanfar, S. Sadiq, Y. Yan, H. Tian, Y. Tao, M. P. Reyes, M. L. Shyu, S. C. Chen, and S. S. Iyengar, "A survey on deep learning: Algorithms, techniques, and applications," ACM Comput. Surv., vol. 51, pp. 92:1-92:36, Sep 2018.

[36] R. Aarthi and S. Harini, "A survey of deep convolutional neural network applications in image processing," International Journal of Pure and Applied Mathematics, vol. 118, no. 7, pp. 185-190, 2018.

[37] H. Zhang, J. E. Fritts, and S. A. Goldman, "Image segmentation evaluation: A survey of unsupervised methods," Comput. Vis. Image Underst., vol. 110, pp. 260-280, May 2008.

[38] U. Montanari, "On the optimal detection of curves in noisy pictures," Commun. ACM, vol. 14, pp. 335-345, May 1971.

[39] N. Zhang and J. Zhu, "A study of x-ray machine image local semantic features extraction model based on bag-ofwords for airport security," International Journal on Smart Sensing and Intelligent Systems, vol. 8, no. 1, pp. 45-64, 2015.

[40] H. Ahmady Phoulady, D. B. Goldgof, L. O. Hall, and P. R. Mouton, "A new approach to detect and segment overlapping cells in multi-layer cervical cell volume images," in 2016 IEEE 13th International Symposium on Biomedical Imaging (ISBI), pp. 201-204, April 2016.

[41] D. Ushizima, A. Bianchi, and C. Carneiro, "Segmentation of subcellular compartiments combining superpixel representation with voronoi diagrams," in Overlapping Cervical Cytology Image Segmentation Challenge, IEEE ISBI, Beijing, China, April 2014, 1st Place, Apr 2014.

[42] H. Lee and J. Kim, "Segmentation of overlapping cervical cells in microscopic images with superpixel partitioning and cell-wise contour refinement," in IEEE Conference on Computer Vision and Pattern Recognition Workshops (CVPRW), pp. 1367-1373, Jun 2016. 
[43] Y. Song, E. L. Tan, X. Jiang, J. Z. Cheng, D. Ni, S. Chen, B. Lei, and T. Wang, “Accurate cervical cell segmentation from overlapping clumps in pap smear images," IEEE Transactions on Medical Imaging, vol. 36, pp. 288-300, Jan 2017.

[44] T. L. Mahyari and R. M. Dansereau, "Deep learning methods for image segmentation containing translucent overlapped objects," in 2019 IEEE Global Conference on Signal and Information Processing (GlobalSIP), pp. 1-5, Nov 2019.

[45] T. L. Mahyari and R. M. Dansereau, "Learning-based multilabel random walks for image segmentation containing translucent overlapped objects," in 2017 40th International Conference on Telecommunications and Signal Processing (TSP), pp. 610-614, July 2017.

[46] T. L. Mahyari and R. M. Dansereau, Multi-layer Random Walker Image Segmentation for Overlapped Cervical Cells using Probabilistic Deep Learning Methods, 2021. Ready to submit, journal paper.

[47] T. L. Mahyari and R. M. Dansereau, "Deep learning methods for image decomposition of cervical cells," in 28th European Signal Processing Conference (EUSIPCO 2020), 2020.

[48] T. L. Mahyari and R. M. Dansereau, Two Step CNN-based method for Overlapped Cervical Cell Decomposition, 2021. Ready to submit, journal paper.

[49] H. W. Kuhn and B. Yaw, "The hungarian method for the assignment problem," in Naval Res. Logist. Quart, pp. 83-97, 1955.

[50] A. Panchbhai, "Wilhelm conrad rontgen and the discovery of x-rays: Revisited after centennial," Journal of Indian Academy of Oral Medicine and Radiology, vol. 27, no. 1, pp. 90-95, 2015.

[51] N. Hättenschwiler, S. Merks, and A. Schwaninger, "Airport security x-ray screening of hold baggage: $2 \mathrm{~d}$ versus $3 \mathrm{~d}$ imaging and evaluation of an on-screen alarm resolution protocol," in 2018 International Carnahan Conference on Security Technology (ICCST), pp. 1-5, 2018.

[52] T. Nyathi, T. Chirwa, and D. van der Merwe, "A survey of digital radiography practice in four south african teaching hospitals: An illuminative study," Biomedical imaging and intervention journal, vol. 6, pp. 1-6, Jan 2010.

[53] M. Güdel, "X-ray astronomy of stellar coronae," The Astronomy and Astrophysics Review, vol. 12, pp. 71-237, Sep 2004. 
[54] S. Mathanker, "X-ray applications in food and agriculture: A review," Transactions of the ASABE (American Society of Agricultural and Biological Engineers), vol. 56, pp. 12271239, Apr 2013.

[55] S. Inkoom, C. Schandorf, G. Emi-Reynolds, and J. J. Fletcher, Quality Assurance and Quality Control of Equipment in Diagnostic Radiology Practice-The Ghanaian Experience. intech, Nov 2011.

[56] J. Wadum, J. Mass, E. Uffelmann, B. Buckley, I. Grimstad, A. Vila, J. Delaney, V. Andrews, L. Burns, S. Florescu, and A. Hull, "Portable x-ray fluorescence and infrared fluorescence imaging studies of cadmium yellow alteration in paintings by edward munch and henri matisse in oslo, copenhagen, and san francisco," SMITHSONIAN CONTRIBUTIONS TO MUSEUM CONSERVATION, pp. 53-64, Oct 2016.

[57] M. A. Al-Abbadi, "Basics of cytology," Avicenna Journal of Medicine, vol. 1, no. 1, pp. 18$28,2011$.

[58] J. A. Delmerico, P. David, and J. J. Corso, "Building façade detection, segmentation and parameter estimation for mobile robot localization and guidance," in International Conference on Intelligent Robots and Systems, pp. 1632-1639-253, 2011.

[59] Y. Ramadevi, T. Sridevi, B. Poornima, and B.Kalyani, "Segmentation and object recognition using edge detection techniques," International Journal of Computer Science and Information Technology (IJCSIT), vol. 2, no. 6, 2010.

[60] W. Wu, A. Y. C. Chen, L. Zhao, and J. J. Corso, "Brain tumor detection and segmentation in a crf framework with pixel-pairwise affinity and super pixel-level features," International Journal of Computer Aided Radiology and Surgery, vol. 9, pp. 241-253, 2014.

[61] S. Deepa and B. Devi, "A survey on artificial intelligence approaches for medical image classification," Indian Journal of Science and Technology, vol. 4, no. 11, pp. 1583-1595, 2011.

[62] J. Shi and J. Malik, "Normalized cuts and image segmentation," IEEE Transactions on Pattern Analysis and Machine Intelligence, vol. 22, no. 8, pp. 888-905, 2000.

[63] Y. Boykov and V. Kolmogorov, "An experimental comparison of mincut/max-flow algorithms for energy minimization in vision," IEEE Transactions on Pattern Analysis and Machine Intelligence, vol. 26, no. Sep, pp. 1124-1137, 2004. 
[64] L. Grady and E. L. Schwartz, "Isoperimetric graph partitioning for image segmentation," IEEE Transactions on Pattern Analysis and Machine Intelligence, vol. 28, no. 3, pp. 469475, 2006.

[65] C. T. Zahn, “Graph-theoretical methods for detecting and describing gestalt clusters,” IEEE Transactions on Computers, vol. 20, no. 1, pp. 68-86, 1971.

[66] S. Geman and D. Geman, "Stochastic relaxation, Gibbs distributions, and the Bayesian restoration of images," IEEE Transactions on Pattern Analisys and Machine Intelligence, vol. 6, pp. 721-741, Nov 1984.

[67] Y. Boykov and M.-P. Jolly, "Interactive graph cuts for optimal boundary and region segmentation of objects in N-D images," in Proc. of International Conference on Computer Vision, pp. 105-112, 2001.

[68] R. Behera and K. Das, "A survey on machine learning: Concept, algorithms and applications," International Journal of Innovative Research in Computer and Communication Engineering, vol. 2, Feb 2017.

[69] L. Deng and D. Yu, "Deep learning: Methods and applications," Foundations and Trends® in Signal Processing, vol. 7, no. 3-4, pp. 197-387, 2014.

[70] Imagenet Large Scale Visual Recognition Challenge 2010 (ILSVRC2010), available on 30 Apr 2021. http: / / www. image-net.org/challenges/LSVRC/2010/.

[71] Imagenet Large Scale Visual Recognition Challenge 2012 (ILSVRC2012), available on 30 Apr 2021. http://www. image-net.org/challenges/LSVRC/2012/.

[72] Imagenet Large Scale Visual Recognition Challenge 2014 (ILSVRC2014), available on 30 Apr 2021. http: //www. image-net.org/challenges/LSVRC/2014/.

[73] J. Dean, G. Corrado, R. Monga, K. Chen, M. Devin, M. Mao, M. A. Ranzato, A. Senior, P. Tucker, K. Yang, Q. V. Le, and A. Y. Ng, "Large scale distributed deep networks," in Advances in Neural Information Processing Systems 25 (F. Pereira, C. J. C. Burges, L. Bottou, and K. Q. Weinberger, eds.), pp. 1223-1231, Curran Associates, Inc., 2012.

[74] E. Shelhamer, J. Long, and T. Darrell, "Fully convolutional networks for semantic segmentation," IEEE Transactions on Pattern Analysis and Machine Intelligence, vol. 39, no. 4, pp. 640-651, 2017. 
[75] M. Jafari, D. Auer, S. Francis, J. Garibaldi, and X. Chen, "Dru-net: An efficient deep convolutional neural network for medical image segmentation," in 2020 IEEE 17th International Symposium on Biomedical Imaging (ISBI), pp. 1144-1148, 2020.

[76] E. Be'ery and A. Yeredor, "Blind separation of superimposed shifted images using parameterized joint diagonalization," IEEE Transactions on Image Processing, vol. 17, no. 3, pp. 340-353, 2008.

[77] K. Hara, K. Inoue, and K. Urahama, "Separation of layers from images containing multiple reflections and transparency using cyclic permutation," in 2009 IEEE International Conference on Acoustics, Speech and Signal Processing, pp. 1157-1160, Apr 2009.

[78] K. Hara, K. Inoue, and K. Urahama, "Generalized mixture ratio based blind image separation,” IEEE Signal Processing Letters, vol. 20, no. 8, pp. 743-746, 2013.

[79] W. Souidene, A. Aissa-El-Bey, K. Abed-Meraim, and A. Beghdadi, "Blind image separation using sparse representation," 2007 IEEE International Conference on Image Processing (ICIP), vol. 3, pp. 125-128, Sep 2007.

[80] B. Ophir and D. Malah, "Show-through cancellation in scanned images using blind source separation techniques," in 2007 IEEE International Conference on Image Processing (ICIP), vol. 3, pp. 233-236, Sep 2007.

[81] O. Tichý and V. Smidl, "Variational blind source separation toolbox and its application to hyperspectral image data," in European Signal Processing Conference (EUSIPCO), pp. 13261330, August-September 2015.

[82] S. Arberet, "Hyper-demix: Blind source separation of hyperspectral images using local ML estimates," in IEEE International Conference on Image Processing (ICIP), pp. 1393-1396, Sep 2010.

[83] Y. Chen, M. Maitre, and T. Fang, "Transparent layer separation for dual energy imaging," in IEEE International Conference on Image Processing (ICIP), pp. 821-824, Oct 2008.

[84] N. Deligiannis, J. F. C. Mota, B. Cornelis, M. R. D. Rodrigues, and I. Daubechies, "X-ray image separation via coupled dictionary learning," in 2016 IEEE International Conference on Image Processing (ICIP), pp. 3533-3537, Sep 2016.

[85] G. Zhou, Z. Yang, S. Xie, and J. M. Yang, "Online blind source separation using incremental nonnegative matrix factorization with volume constraint," IEEE Transactions on Neural Networks, vol. 22, no. 4, pp. 550-560, 2011. 
[86] Y. Qian, S. Jia, J. Zhou, and A. Robles-Kelly, "Hyperspectral unmixing via 11/2 sparsityconstrained nonnegative matrix factorization," IEEE Transactions on Geoscience and Remote Sensing, vol. 49, no. 11, pp. 4282-4297, 2011.

[87] W. Wang, Y. Qian, and Y. Y. Tang, "Hypergraph-regularized sparse nmf for hyperspectral unmixing," IEEE Journal of Selected Topics in Applied Earth Observations and Remote Sensing, vol. 9, no. 2, pp. 681-694, 2016.

[88] D. Cai, X. He, J. Han, and T. S. Huang, "Graph regularized nonnegative matrix factorization for data representation," IEEE Transactions on Pattern Analysis and Machine Intelligence, vol. 33, no. 8, pp. 1548-1560, 2011.

[89] J. J. Y. Wang, H. Bensmail, and X. Gao, "Multiple graph regularized nonnegative matrix factorization," Pattern Recogn., vol. 46, no. 10, pp. 2840-2847, 2013.

[90] L. Tong, J. Zhou, Y. Qian, and Y. Gao, "Multiple graph regularized NMF for hyperspectral unmixing," in 2015 7th Workshop on Hyperspectral Image and Signal Processing: Evolution in Remote Sensing (WHISPERS), pp. 1-4, June 2015.

[91] J. Rapin, J. Bobin, A. Larue, and J. L. Starck, "Nmf with sparse regularizations in transformed domains," SIAM J. Imaging Sciences, vol. 7, no. 4, pp. 2020-2047, 2014.

[92] R. Zdunek and A. Cichocki, "Blind image separation using nonnegative matrix factorization with gibbs smoothing," in proceedings of ICONIP 2007, Revised Selected Papers, Part II, pp. 519-528, Springer Berlin Heidelberg, 2008.

[93] N. Xu, B. Price, S. Cohen, and T. Huang, "Deep image matting," in 2017 IEEE Conference on Computer Vision and Pattern Recognition (CVPR), pp. 311-320, 2017.

[94] C. Dong, X. Zeng, L. Lin, H. Hu, X. Han, M. Naghedolfeizi, D. Aberra, and Y. W. Chen, “An improved random walker with bayes model for volumetric medical image segmentation," Journal of Healthcare Engineering, vol. 2017, 2017.

[95] S. Andrews, G. Hamarneh, and A. Saad, "Fast random walker with priors using precomputation for interactive medical image segmentation," in Medical Image Computing and Computer-Assisted Intervention - MICCAI 2010, pp. 9-16, 2010.

[96] B-spline Grid, Image and Point based Registration, available on 30 Apr 2021. https://www.mathworks.com/matlabcentral/fileexchange/ 20057-b-spline-grid-image-and-point-based-registration. 
[97] E. Orhan and X. Pitkow, "Skip connections eliminate singularities," in International Conference on Learning Representations, 2018.

[98] S. Mei, A. Montanari, and P. M. Nguyen, "A mean field view of the landscape of two-layer neural networks,” Proc Natl Acad Sci U S A, vol. 115, no. 33, pp. E7665-E7671, 2018.

[99] G. Hinton, Neural Networks for Machine Learning - Lecture 6a - Overview of mini-batch gradient descent., 2012, available on 30 Apr 2021. http://www.cs.toronto.edu/ tijmen/csc321/slides/lecture_slides_lec6.pdf.

[100] D. P. Kingma and J. Ba, "Adam: A method for stochastic optimization," in $\underline{\text { 3rd International }}$ Conference on Learning Representations, ICLR 2015, San Diego, CA, USA, May 7-9, 2015, Conference Track Proceedings, 2015.

[101] O. Ronneberger, P. Fischer, and T. Brox, "U-net: Convolutional networks for biomedical image segmentation," pp. 234-241, 2015.

[102] A. Rosenfeld and J. L. Pfaltz, "Sequential operations in digital picture processing," Journal of the ACM, vol. 13, no. 4, pp. 471-494, 1966.

[103] Sharcnet Cluster, available on 30 Apr 2021. https://www.sharcnet.ca/my/ front/.

[104] Z. Wang, A. C. Bovik, H. R. Sheikh, and E. P. Simoncelli, "Image quality assessment: From error visibility to structural similarity," IEEE Transactions on Image Processing, vol. 13, no. 4, pp. 600-612, 2004.

[105] Z. Kato and T. C. Pong, "A Markov random field image segmentation model for color textured images," Image and Vision Computing, vol. 24, pp. 1103-1114, Oct. 2006.

[106] R. S. Sutton, "Two problems with backpropagation and other steepest-descent learning procedures for networks," in Proceedings of the Eighth Annual Conference of the Cognitive Science Society, Hillsdale, NJ: Erlbaum, 1986.

[107] N. Qian, "On the momentum term in gradient descent learning algorithms," Neural Networks, vol. 12, pp. 145-151, Jan 1999.

[108] J. C. Duchi, E. Hazan, and Y. Singer, "Adaptive subgradient methods for online learning and stochastic optimization.,” J. Mach. Learn. Res., vol. 12, pp. 2121-2159, 2011. 
[109] S. R. Dubey, S. Chakraborty, S. K. Roy, S. Mukherjee, S. K. Singh, and B. B. Chaudhuri, "diffgrad: An optimization method for convolutional neural networks," IEEE Transactions on Neural Networks and Learning Systems, vol. 31, no. 11, pp. 4500-4511, 2020.

[110] T. Dozat, "Incorporating nesterov momentum into adam,” in ICLR workshop, pp. 1-4, 2016. 


\section{Appendix A}

\section{Convolutional Neural Networks Structures}

\section{A.1 SegNet Network}

Tables (A.1-A.8) explain SegNet network [12] with 91 layers.

\section{A.2 Residual Network for Overlapped Image Segmentation}

The details of our proposed residual network with 81 layers is come in Tables (A.9-??).

\section{A.3 Residual Network for Image Decomposition}

For image decomposition we designed a residual network with 66 layers that are explained in Tables (A.16-A.21). 
Table A.1: SegNet, layers 1-12.

\begin{tabular}{|c|c|c|c|}
\hline Layer Number & Layer Name & Layer Type & Layer Description \\
\hline \multirow[t]{2}{*}{1} & 'inputImage' & Image Input & $360 \times 480 \times 3$ images with \\
\hline & & & 'zerocenter' normalization \\
\hline \multirow[t]{2}{*}{2} & 'conv $11_{1} 1^{\prime}$ & Convolution & $643 \times 3 \times 3$ convolutions with \\
\hline & & & stride $\left[\begin{array}{ll}1 & 1\end{array}\right]$, padding $\left[\begin{array}{llll}1 & 1 & 1 & 1\end{array}\right]$ \\
\hline 3 & 'bn_conv1_1' & Batch Normalization & Batch normalization \\
\hline 4 & 'relu1_1' & ReLU & ReLU \\
\hline \multirow[t]{2}{*}{5} & 'conv1_2' & Convolution & $643 \times 3 \times 64$ convolutions with \\
\hline & & & stride $\left[\begin{array}{ll}1 & 1\end{array}\right]$, padding $\left[\begin{array}{llll}1 & 1 & 1 & 1\end{array}\right]$ \\
\hline 6 & 'bn_conv1_2' & Batch Normalization & Batch normalization \\
\hline 7 & 'relu1_2' & ReLU & ReLU \\
\hline \multirow[t]{2}{*}{8} & 'pool1' & Max Pooling & $2 \times 2$ max pooling with stride \\
\hline & & & {$\left[\begin{array}{ll}2 & 2\end{array}\right]$, padding $\left[\begin{array}{llll}0 & 0 & 0 & 0\end{array}\right]$} \\
\hline \multirow[t]{2}{*}{9} & 'conv $21_{-}{ }^{\prime}$ & Convolution & $1283 \times 3 \times 64$ convolutions with stride \\
\hline & & & {$\left[\begin{array}{ll}1 & 1\end{array}\right]$ and padding $\left[\begin{array}{llll}1 & 1 & 1 & 1\end{array}\right]$} \\
\hline 10 & 'bn_conv2_1' & Batch Normalization & Batch normalization \\
\hline 11 & 'relu2_1' & ReLU & ReLU \\
\hline \multirow[t]{2}{*}{12} & 'conv2_2' & Convolution & $1283 \times 3 \times 128$ convolutions with \\
\hline & & & stride $\left[\begin{array}{ll}1 & 1\end{array}\right]$, padding $\left[\begin{array}{llll}1 & 1 & 1 & 1\end{array}\right]$ \\
\hline
\end{tabular}


Table A.2: SegNet, layers 13-24.

\begin{tabular}{|c|c|c|c|}
\hline Layer Number & Layer Name & Layer Type & Layer Description \\
\hline 13 & 'bn_conv2_2' & Batch Normalization & Batch normalization \\
\hline 14 & 'relu2_2' & ReLU & ReLU \\
\hline \multirow[t]{2}{*}{15} & 'pool2' & Max Pooling & $2 \times 2$ max pooling with stride \\
\hline & & & {$\left[\begin{array}{ll}2 & 2\end{array}\right]$, padding $\left[\begin{array}{llll}0 & 0 & 0 & 0\end{array}\right]$} \\
\hline \multirow[t]{2}{*}{16} & 'conv3_1' & Convolution & $2563 \times 3 \times 128$ convolutions with stride \\
\hline & & & {$\left[\begin{array}{ll}1 & 1\end{array}\right]$ and padding $\left[\begin{array}{llll}1 & 1 & 1 & 1\end{array}\right]$} \\
\hline 17 & 'bn_conv3_1' & Batch Normalization & Batch normalization \\
\hline 18 & 'relu3_1' & ReLU & ReLU \\
\hline \multirow[t]{2}{*}{19} & 'conv $3 \_2^{\prime}$ & Convolution & $2563 \times 3 \times 256$ convolutions with \\
\hline & & & stride $\left[\begin{array}{ll}1 & 1\end{array}\right]$, padding $\left[\begin{array}{llll}1 & 1 & 1 & 1\end{array}\right]$ \\
\hline 20 & 'bn_conv3_2' & Batch Normalization & Batch normalization \\
\hline 21 & 'relu3_2' & ReLU & ReLU \\
\hline \multirow[t]{2}{*}{22} & 'conv3_3' & Convolution & $2563 \times 3 \times 256$ convolutions with stride \\
\hline & & & {$\left[\begin{array}{ll}1 & 1\end{array}\right]$, padding $\left[\begin{array}{llll}1 & 1 & 1 & 1\end{array}\right]$} \\
\hline 23 & 'bn_conv3_3' & Batch Normalization & Batch normalization \\
\hline 24 & 'relu3_3' & ReLU & ReLU \\
\hline
\end{tabular}


Table A.3: SegNet, layers 25-36.

\begin{tabular}{|c|c|c|c|}
\hline Layer Number & Layer Name & Layer Type & Layer Description \\
\hline \multirow[t]{2}{*}{25} & 'pool3' & Max Pooling & $2 \times 2$ max pooling with stride \\
\hline & & & {$\left[\begin{array}{ll}2 & 2\end{array}\right]$, padding $\left[\begin{array}{llll}0 & 0 & 0 & 0\end{array}\right]$} \\
\hline \multirow[t]{2}{*}{26} & 'conv4_1' & Convolution & $5123 \times 3 \times 256$ convolutions with \\
\hline & & & stride [1 1 , padding [ $\left[\begin{array}{llll}1 & 1 & 1 & 1\end{array}\right]$ \\
\hline 27 & 'bn_conv4_1' & Batch Normalization & Batch normalization \\
\hline 28 & 'relu4' & ReLU & $\operatorname{ReLU}$ \\
\hline \multirow[t]{2}{*}{29} & 'conv $4 \_2$ ' & Convolution & $5123 \times 3 \times 512$ convolutions with \\
\hline & & & stride $\left[\begin{array}{ll}1 & 1\end{array}\right]$, padding $\left[\begin{array}{llll}1 & 1 & 1 & 1\end{array}\right]$ \\
\hline 30 & 'bn_conv $4 \_2 '$ & Batch Normalization & Batch normalization \\
\hline 31 & 'relu4 $4_{2}^{\prime}$ & ReLU & ReLU \\
\hline \multirow[t]{2}{*}{32} & 'conv4_3' & Convolution & $5123 \times 3 \times 512$ convolutions with \\
\hline & & & stride $\left[\begin{array}{ll}1 & 1\end{array}\right]$, padding $\left[\begin{array}{llll}1 & 1 & 1 & 1\end{array}\right]$ \\
\hline 33 & 'bn_conv4_3' & Batch Normalization & Batch normalization \\
\hline 34 & 'relu4_3' & ReLU & ReLU \\
\hline \multirow[t]{2}{*}{35} & 'pool4' & Max Pooling & $2 \times 2$ max pooling with stride \\
\hline & & & {$\left[\begin{array}{ll}2 & 2\end{array}\right]$, padding $\left[\begin{array}{llll}0 & 0 & 0 & 0\end{array}\right]$} \\
\hline \multirow[t]{2}{*}{36} & 'conv $5 \_1$ ' & Convolution & $5123 \times 3 \times 512$ convolutions with \\
\hline & & & stride $\left[\begin{array}{ll}1 & 1\end{array}\right]$, padding $\left[\begin{array}{llll}1 & 1 & 1 & 1\end{array}\right]$ \\
\hline
\end{tabular}


Table A.4: SegNet, layers 37-48.

\begin{tabular}{|c|c|c|c|}
\hline Layer Number & Layer Name & Layer Type & Layer Description \\
\hline 37 & 'bn_conv5_1' & Batch Normalization & Batch normalization \\
\hline 38 & 'relu5_1' & ReLU & ReLU \\
\hline \multirow[t]{2}{*}{39} & 'conv $5 \_2$ ' & Convolution & $5123 \times 3 \times 512$ convolutions with \\
\hline & & & stride $\left[\begin{array}{ll}1 & 1\end{array}\right]$, padding $\left[\begin{array}{llll}1 & 1 & 1 & 1\end{array}\right]$ \\
\hline 40 & 'bn_conv5_2' & Batch Normalization & Batch normalization \\
\hline 41 & 'relu5_2' & ReLU & ReLU \\
\hline \multirow[t]{2}{*}{42} & 'conv $5 \_3$ ' & Convolution & $5123 \times 3 \times 512$ convolutions with \\
\hline & & & stride $\left[\begin{array}{ll}1 & 1\end{array}\right]$, padding $\left[\begin{array}{llll}1 & 1 & 1 & 1\end{array}\right]$ \\
\hline 43 & 'bn_conv5_3' & Batch Normalization & Batch normalization \\
\hline 44 & 'relu5_3' & ReLU & ReLU \\
\hline \multirow[t]{2}{*}{45} & 'pool5' & Max Pooling & $2 \times 2$ max pooling with stride \\
\hline & & & {$\left[\begin{array}{ll}2 & 2\end{array}\right]$, padding $\left[\begin{array}{llll}0 & 0 & 0 & 0\end{array}\right]$} \\
\hline 46 & 'decoder5_unpool' & Max Unpooling & Max Unpooling \\
\hline \multirow[t]{2}{*}{47} & 'decoder5_conv3' & Convolution & $5123 \times 3 \times 512$ convolutions with \\
\hline & & & stride $\left[\begin{array}{ll}1 & 1\end{array}\right]$, padding $\left[\begin{array}{llll}1 & 1 & 1 & 1\end{array}\right]$ \\
\hline 48 & 'decoder $5 \_b n \_3$ ' & Batch Normalization & Batch normalization \\
\hline
\end{tabular}


Table A.5: SegNet, layers 49-60.

\begin{tabular}{|c|c|c|c|}
\hline Layer Number & Layer Name & Layer Type & Layer Description \\
\hline 49 & 'decoder5_relu ${ }_{3}^{\prime}$ & ReLU & ReLU \\
\hline \multirow[t]{2}{*}{50} & 'decoder5_conv2' & Convolution & $5123 \times 3 \times 512$ convolutions with \\
\hline & & & stride $\left[\begin{array}{ll}1 & 1\end{array}\right]$, padding $\left[\begin{array}{llll}1 & 1 & 1 & 1\end{array}\right]$ \\
\hline 51 & 'decoder5_bn_2' & Batch Normalization & Batch normalization \\
\hline 52 & 'decoder5_relu_2' & ReLU & ReLU \\
\hline \multirow[t]{2}{*}{53} & 'decoder5_conv1' & Convolution & $5123 \times 3 \times 512$ convolutions with \\
\hline & & & stride $\left[\begin{array}{ll}1 & 1\end{array}\right]$, padding $\left[\begin{array}{llll}1 & 1 & 1 & 1\end{array}\right]$ \\
\hline 54 & 'decoder5_bn_1' & Batch Normalization & Batch normalization \\
\hline 55 & 'decoder5_relu_1' & ReLU & ReLU \\
\hline 56 & 'decoder4_unpool' & Max Unpooling & Max Unpooling \\
\hline \multirow[t]{2}{*}{57} & 'decoder4_conv3' & Convolution & $5123 \times 3 \times 512$ convolutions with \\
\hline & & & stride $\left[\begin{array}{ll}1 & 1\end{array}\right]$, padding $\left[\begin{array}{llll}1 & 1 & 1 & 1\end{array}\right]$ \\
\hline 58 & 'decoder4_bn_3' & Batch Normalization & Batch normalization \\
\hline 59 & 'decoder4_relu_3' & $\operatorname{ReLU}$ & ReLU \\
\hline \multirow[t]{2}{*}{60} & 'decoder4_conv2' & Convolution & $5123 \times 3 \times 512$ convolutions with \\
\hline & & & stride $\left[\begin{array}{ll}1 & 1\end{array}\right]$, padding $\left[\begin{array}{llll}1 & 1 & 1 & 1\end{array}\right]$ \\
\hline
\end{tabular}


Table A.6: SegNet, layers 61-72.

\begin{tabular}{|c|c|c|c|}
\hline Layer Number & Layer Name & Layer Type & Layer Description \\
\hline 61 & 'decoder4_bn_2' & Batch Normalization & Batch normalization \\
\hline 62 & 'decoder4_relu_2' & ReLU & ReLU \\
\hline \multirow[t]{2}{*}{63} & 'decoder4_conv1' & Convolution & $2563 \times 3 \times 512$ convolutions with stride \\
\hline & & & {$\left[\begin{array}{ll}1 & 1\end{array}\right]$, padding $\left[\begin{array}{llll}1 & 1 & 1 & 1\end{array}\right]$} \\
\hline 64 & 'decoder4_bn_1' & Batch Normalization & Batch normalization \\
\hline 65 & 'decoder4_relu_1' & ReLU & ReLU \\
\hline 66 & 'decoder3_unpool' & Max Unpooling & Max Unpooling \\
\hline \multirow[t]{2}{*}{67} & 'decoder3_conv3' & Convolution & $2563 \times 3 \times 256$ convolutions with \\
\hline & & & stride $\left[\begin{array}{ll}1 & 1\end{array}\right]$, padding $\left[\begin{array}{llll}1 & 1 & 1 & 1\end{array}\right]$ \\
\hline 68 & 'decoder3_bn_3' & Batch Normalization & Batch normalization \\
\hline 69 & 'decoder3_relu_3' & $\operatorname{ReLU}$ & ReLU \\
\hline \multirow[t]{2}{*}{70} & 'decoder3_conv2' & Convolution & $2563 \times 3 \times 256$ convolutions with \\
\hline & & & stride $\left[\begin{array}{ll}1 & 1\end{array}\right]$, padding $\left[\begin{array}{llll}1 & 1 & 1 & 1\end{array}\right]$ \\
\hline 71 & 'decoder $3 \_b n \_2$ ' & Batch Normalization & Batch normalization \\
\hline 72 & 'decoder3_relu_2' & ReLU & ReLU \\
\hline
\end{tabular}


Table A.7: SegNet, layers 73-84.

\begin{tabular}{|c|c|c|c|}
\hline Layer Number & Layer Name & Layer Type & Layer Description \\
\hline \multirow[t]{2}{*}{73} & 'decoder3_conv1' & Convolution & $1283 \times 3 \times 256$ convolutions with \\
\hline & & & stride $\left[\begin{array}{ll}1 & 1\end{array}\right]$, padding $\left[\begin{array}{llll}1 & 1 & 1 & 1\end{array}\right]$ \\
\hline 74 & 'decoder $3 \_b n \_1$ 1' & Batch Normalization & Batch normalization \\
\hline 75 & 'decoder3_relu_1' & ReLU & ReLU \\
\hline 76 & 'decoder $2 \_$unpool' & Max Unpooling & Max Unpooling \\
\hline \multirow[t]{2}{*}{77} & 'decoder2_conv2' & Convolution & $1283 \times 3 \times 128$ convolutions with \\
\hline & & & stride $\left[\begin{array}{ll}1 & 1\end{array}\right]$, padding $\left[\begin{array}{llll}1 & 1 & 1 & 1\end{array}\right]$ \\
\hline 78 & 'decoder $2 \_b n \_2$ ' & Batch Normalization & Batch normalization \\
\hline 79 & 'decoder $2 \_r e l u \_2$ ' & ReLU & ReLU \\
\hline \multirow[t]{2}{*}{80} & 'decoder2_conv1' & Convolution & $643 \times 3 \times 128$ convolutions with \\
\hline & & & stride $\left[\begin{array}{ll}1 & 1\end{array}\right]$, padding $\left[\begin{array}{llll}1 & 1 & 1 & 1\end{array}\right]$ \\
\hline 81 & 'decoder $2 \_b n \_1$ ' & Batch Normalization & Batch normalization \\
\hline 82 & 'decoder2_relu_1' & ReLU & ReLU \\
\hline 83 & 'decoder1_unpool' & Max Unpooling & Max Unpooling \\
\hline 84 & 'decoder1_conv2' & Convolution & $643 \times 3 \times 64$ convolutions with \\
\hline & & & stride $\left[\begin{array}{ll}1 & 1\end{array}\right]$, padding $\left[\begin{array}{llll}1 & 1 & 1 & 1\end{array}\right]$ \\
\hline
\end{tabular}


Table A.8: SegNet, layers 85-91.

\begin{tabular}{|c|c|c|c|}
\hline Layer Number & Layer Name & Layer Type & Layer Description \\
\hline 85 & 'decoder $1 \_b n \_2$ ' & Batch Normalization & Batch normalization \\
\hline 86 & 'decoder1_relu_2' & ReLU & ReLU \\
\hline \multirow[t]{2}{*}{87} & 'decoder1_conv1' & Convolution & $43 \times 3 \times 64$ convolutions with \\
\hline & & & stride $\left[\begin{array}{ll}1 & 1\end{array}\right]$, padding $\left[\begin{array}{llll}1 & 1 & 1 & 1\end{array}\right]$ \\
\hline 88 & 'decoder $1 \_b n \_1$ ' & Batch Normalization & Batch normalization \\
\hline 89 & 'decoder1_relu_1' & ReLU & ReLU \\
\hline 90 & 'softmax' & Softmax & softmax \\
\hline 91 & 'labels' & Pixel Classification Layer & Class weighted cross-entropy \\
\hline & & & loss with 'Obj1', .., 'Obj4' \\
\hline
\end{tabular}


Table A.9: Proposed residual network for image segmentation with 81 layers, layers 1-12.

\begin{tabular}{|c|c|c|c|}
\hline Layer Number & Layer Name & Layer Type & Layer Description \\
\hline \multirow[t]{2}{*}{1} & 'inputImage' & Image Input & $200 \times 200 \times 3$ images with \\
\hline & & & 'zerocenter' normalization \\
\hline \multirow[t]{2}{*}{2} & 'conv1_1' & Convolution & $643 \times 3 \times 3$ convolutions with \\
\hline & & & stride $\left[\begin{array}{ll}1 & 1\end{array}\right]$, padding $\left[\begin{array}{llll}1 & 1 & 1 & 1\end{array}\right]$ \\
\hline 3 & 'bn_conv1_1' & Batch Normalization & Batch normalization \\
\hline 4 & 'relu1_1' & ReLU & ReLU \\
\hline \multirow[t]{2}{*}{5} & 'conv $1 \_2$ ' & Convolution & $643 \times 3 \times 64$ convolutions with \\
\hline & & & stride $\left[\begin{array}{ll}1 & 1\end{array}\right]$, padding $\left[\begin{array}{llll}1 & 1 & 1 & 1\end{array}\right]$ \\
\hline 6 & 'bn_conv1_2' & Batch Normalization & Batch normalization \\
\hline 7 & 'relu1_2' & ReLU & ReLU \\
\hline \multirow[t]{2}{*}{8} & 'pool1' & Max Pooling & $2 \times 2$ max pooling with stride \\
\hline & & & {$\left[\begin{array}{ll}2 & 2\end{array}\right]$, padding $\left[\begin{array}{llll}0 & 0 & 0 & 0\end{array}\right]$} \\
\hline \multirow[t]{2}{*}{9} & 'conv2_1' & Convolution & $1283 \times 3 \times 64$ convolutions with \\
\hline & & & stride $\left[\begin{array}{ll}1 & 1\end{array}\right]$, padding $\left[\begin{array}{llll}1 & 1 & 1 & 1\end{array}\right]$ \\
\hline 10 & 'bn_conv2_1' & Batch Normalization & Batch normalization \\
\hline 11 & 'relu2_1' & $\operatorname{ReLU}$ & ReLU \\
\hline \multirow[t]{2}{*}{12} & 'conv2_2' & Convolution & $1283 \times 3 \times 128$ convolutions with \\
\hline & & & stride $\left[\begin{array}{ll}1 & 1\end{array}\right]$, padding $\left[\begin{array}{llll}1 & 1 & 1 & 1\end{array}\right]$ \\
\hline
\end{tabular}


Table A.10: Proposed residual network for image segmentation, layers 13-24.

\begin{tabular}{|c|c|c|c|}
\hline Layer Number & Layer Name & Layer Type & Layer Description \\
\hline 13 & 'bn_conv2_2' & Batch Normalization & Batch normalization \\
\hline \multirow[t]{2}{*}{14} & $' a d d_{c} 2^{\prime}$ & Addition & Element-wise addition of 2 inputs \\
\hline & & & conv2_1 and $b n_{-}$conv2_2 \\
\hline 15 & 'relu2_2' & ReLU & ReLU \\
\hline \multirow[t]{2}{*}{16} & 'pool2' & Max Pooling & $2 \times 2$ max pooling with stride \\
\hline & & & {$\left[\begin{array}{ll}2 & 2\end{array}\right]$, padding $\left[\begin{array}{llll}0 & 0 & 0 & 0\end{array}\right]$} \\
\hline \multirow[t]{2}{*}{17} & 'conv $3 \_1$ ' & Convolution & $2563 \times 3 \times 128$ convolutions with \\
\hline & & & stride $\left[\begin{array}{ll}1 & 1\end{array}\right]$, padding $\left[\begin{array}{llll}1 & 1 & 1 & 1\end{array}\right]$ \\
\hline 18 & 'bn_conv3_1' & Batch Normalization & Batch normalization \\
\hline 19 & 'relu3_1' & ReLU & ReLU \\
\hline \multirow[t]{2}{*}{20} & 'conv3_2' & Convolution & $2563 \times 3 \times 256$ convolutions with \\
\hline & & & stride $\left[\begin{array}{ll}1 & 1\end{array}\right]$, padding $\left[\begin{array}{llll}1 & 1 & 1 & 1\end{array}\right]$ \\
\hline 21 & 'bn_conv3_2' & Batch Normalization & Batch normalization \\
\hline 22 & 'relu3_2' & ReLU & ReLU \\
\hline \multirow[t]{2}{*}{23} & 'conv $3 \_3{ }^{\prime}$ & Convolution & $2563 \times 3 \times 256$ convolutions with \\
\hline & & & stride $\left[\begin{array}{ll}1 & 1\end{array}\right]$, padding $\left[\begin{array}{llll}1 & 1 & 1 & 1\end{array}\right]$ \\
\hline 24 & 'bn_conv3_3' & Batch Normalization & Batch normalization \\
\hline
\end{tabular}


Table A.11: Proposed residual network for image segmentation, layers 25-36.

\begin{tabular}{|c|c|c|c|}
\hline Layer Number & Layer Name & Layer Type & Layer Description \\
\hline \multirow[t]{2}{*}{25} & $' a d d_{c} 3^{\prime}$ & Addition & Element-wise addition of 2 inputs \\
\hline & & & conv3_1 and bn_conv3_3 \\
\hline 26 & 'relu3_3' & $\operatorname{ReLU}$ & ReLU \\
\hline \multirow[t]{2}{*}{27} & 'pool3' & Max Pooling & $2 \times 2$ max pooling with stride \\
\hline & & & {$\left[\begin{array}{ll}2 & 2\end{array}\right]$, padding $\left[\begin{array}{llll}0 & 0 & 0 & 0\end{array}\right]$} \\
\hline \multirow[t]{2}{*}{28} & 'conv $4_{-} 1^{\prime}$ & Convolution & $5123 \times 3 \times 256$ convolutions with \\
\hline & & & stride $\left[\begin{array}{ll}1 & 1\end{array}\right]$, padding $\left[\begin{array}{llll}1 & 1 & 1 & 1\end{array}\right]$ \\
\hline 29 & 'bn_conv4_1' & Batch Normalization & Batch normalization \\
\hline 30 & 'relu4_1' & ReLU & ReLU \\
\hline \multirow[t]{2}{*}{31} & 'conv $4 \_2$ ' & Convolution & $5123 \times 3 \times 512$ convolutions with \\
\hline & & & stride $\left[\begin{array}{ll}1 & 1\end{array}\right]$, padding $\left[\begin{array}{llll}1 & 1 & 1 & 1\end{array}\right]$ \\
\hline 32 & 'bn_conv4 $\_2^{\prime}$ & Batch Normalization & Batch normalization \\
\hline 33 & 'relu4_2' & $\operatorname{ReLU}$ & $\operatorname{ReLU}$ \\
\hline \multirow[t]{2}{*}{34} & 'conv4_3' & Convolution & $5123 \times 3 \times 512$ convolutions with \\
\hline & & & stride $\left[\begin{array}{ll}1 & 1\end{array}\right]$, padding $\left[\begin{array}{llll}1 & 1 & 1 & 1\end{array}\right]$ \\
\hline 35 & 'bn_conv4_3' & Batch Normalization & Batch normalization \\
\hline 36 & 'add_ct' & Addition & Element-wise addition of 2 inputs \\
\hline & & & conv4_1 and bn_conv4_3 \\
\hline
\end{tabular}


Table A.12: Proposed residual network for image segmentation, layers 37-47.

\begin{tabular}{|c|c|c|c|}
\hline Layer Number & Layer Name & Layer Type & Layer Description \\
\hline 37 & 'relu4_3' & ReLU & ReLU \\
\hline \multirow[t]{2}{*}{38} & 'decoder4_conv3' & Convolution & $5123 \times 3 \times 512$ convolutions with \\
\hline & & & stride $\left[\begin{array}{ll}1 & 1\end{array}\right]$, padding $\left[\begin{array}{llll}1 & 1 & 1 & 1\end{array}\right]$ \\
\hline 39 & 'decoder $4 \_b n \_3$ ' & Batch Normalization & Batch normalization \\
\hline 40 & 'decoder4_relu_3' & ReLU & ReLU \\
\hline \multirow[t]{2}{*}{41} & 'decoder4_conv2' & Convolution & $5123 \times 3 \times 512$ convolutions with \\
\hline & & & stride $\left[\begin{array}{ll}1 & 1\end{array}\right]$, padding $\left[\begin{array}{llll}1 & 1 & 1 & 1\end{array}\right]$ \\
\hline 42 & 'decoder $4 \_b n \_2$ ' & Batch Normalization & Batch normalization \\
\hline 43 & 'decoder4_relu_2' & ReLU & ReLU \\
\hline \multirow[t]{2}{*}{44} & 'decoder4_conv1' & Convolution & $2563 \times 3 \times 512$ convolutions with \\
\hline & & & stride $\left[\begin{array}{ll}1 & 1\end{array}\right]$, padding $\left[\begin{array}{llll}1 & 1 & 1 & 1\end{array}\right]$ \\
\hline 45 & 'decoder4_bn_1' & Batch Normalization & Batch normalization \\
\hline \multirow[t]{2}{*}{46} & 'add_d $d 4^{\prime}$ & Addition & Element-wise addition of 2 inputs \\
\hline & & & 'decoder4_conv3' and 'decoder4_bn_1' \\
\hline 47 & 'decoder4_relu_1' & ReLU & ReLU \\
\hline
\end{tabular}


Table A.13: Proposed residual network for image segmentation, layers 48-58.

\begin{tabular}{|c|c|c|c|}
\hline Layer Number & Layer Name & Layer Type & Layer Description \\
\hline 48 & 'decoder3_unpool' & Max Unpooling & Max Unpooling \\
\hline \multirow[t]{2}{*}{49} & 'decoder3_conv3' & Convolution & $2563 \times 3 \times 256$ convolutions with \\
\hline & & & stride $\left[\begin{array}{ll}1 & 1\end{array}\right]$, padding $\left[\begin{array}{llll}1 & 1 & 1 & 1\end{array}\right]$ \\
\hline 50 & 'decoder $3 \_b n \_3$ ' & Batch Normalization & Batch normalization \\
\hline 51 & 'decoder3_relu_3' & ReLU & ReLU \\
\hline \multirow[t]{2}{*}{52} & 'decoder3_conv2' & Convolution & $2563 \times 3 \times 256$ convolutions with \\
\hline & & & stride $\left[\begin{array}{ll}1 & 1\end{array}\right]$, padding $\left[\begin{array}{llll}1 & 1 & 1 & 1\end{array}\right]$ \\
\hline 53 & 'decoder $3 \_b n \_2$ ' & Batch Normalization & Batch normalization \\
\hline 54 & 'decoder3_relu_2' & ReLU & ReLU \\
\hline \multirow[t]{2}{*}{55} & 'decoder3_conv1' & Convolution & $1283 \times 3 \times 256$ convolutions with \\
\hline & & & stride $\left[\begin{array}{ll}1 & 1\end{array}\right]$, padding $\left[\begin{array}{llll}1 & 1 & 1 & 1\end{array}\right]$ \\
\hline 56 & 'decoder $3 \_b n_{-} 1^{\prime}$ & Batch Normalization & Batch normalization \\
\hline \multirow[t]{2}{*}{57} & 'add_d $d 3^{\prime}$ & Addition & Element-wise addition of 2 inputs \\
\hline & & & 'decoder3_conv3' and 'decoder $3 \_b n_{-} 1^{\prime}$ \\
\hline 58 & 'decoder3_relu_1' & ReLU & ReLU \\
\hline
\end{tabular}


Table A.14: Proposed residual network for image segmentation, layers 59-70.

\begin{tabular}{|c|c|c|c|}
\hline Layer Number & Layer Name & Layer Type & Layer Description \\
\hline 59 & 'decoder2_unpool' & Max Unpooling & Max Unpooling \\
\hline \multirow[t]{2}{*}{60} & 'decoder2_conv2' & Convolution & $1283 \times 3 \times 128$ convolutions with \\
\hline & & & stride $\left[\begin{array}{ll}1 & 1\end{array}\right]$, padding $\left[\begin{array}{llll}1 & 1 & 1 & 1\end{array}\right]$ \\
\hline 61 & 'decoder $2 \_b n \_2$ ' & Batch Normalization & Batch normalization \\
\hline 62 & 'decoder2_relu_2' & ReLU & ReLU \\
\hline \multirow[t]{2}{*}{63} & 'decoder2_conv1' & Convolution & $643 \times 3 \times 128$ convolutions with \\
\hline & & & stride $\left[\begin{array}{ll}1 & 1\end{array}\right]$, padding $\left[\begin{array}{llll}1 & 1 & 1 & 1\end{array}\right]$ \\
\hline 64 & 'decoder $2 \_b n \_1$ ' & Batch Normalization & Batch normalization \\
\hline \multirow[t]{2}{*}{65} & 'add_d $d 2^{\prime}$ & Addition & Element-wise addition of 2 inputs \\
\hline & & & 'decoder2_conv2' and 'decoder2_bn_1' \\
\hline 66 & 'decoder2_relu_1' & ReLU & ReLU \\
\hline 67 & 'decoder1_unpool' & Max Unpooling & Max Unpooling \\
\hline \multirow[t]{2}{*}{68} & 'decoder1_conv2' & Convolution & $643 \times 3 \times 64$ convolutions with \\
\hline & & & stride $\left[\begin{array}{ll}1 & 1\end{array}\right]$, padding $\left[\begin{array}{llll}1 & 1 & 1 & 1\end{array}\right]$ \\
\hline 69 & 'decoder1_bn_2' & Batch Normalization & Batch normalization \\
\hline 70 & 'decoder1_relu_2' & ReLU & ReLU \\
\hline
\end{tabular}


Table A.15: Proposed residual network for image segmentation, layers 71-81.

\begin{tabular}{|c|c|c|c|}
\hline Layer Number & Layer Name & Layer Type & Layer Description \\
\hline \multirow[t]{2}{*}{71} & 'decoder1_conv1' & Convolution & $43 \times 3 \times 64$ convolutions with \\
\hline & & & stride $\left[\begin{array}{ll}1 & 1\end{array}\right]$, padding $\left[\begin{array}{llll}1 & 1 & 1 & 1\end{array}\right]$ \\
\hline 72 & 'decoder1_bn_1' & Batch Normalization & Batch normalization \\
\hline 73 & 'decoder1_relu_1' & ReLU & ReLU \\
\hline 74 & 'sigmoid' & Sigmoid & sigmoid \\
\hline \multirow[t]{2}{*}{75} & 'labels' & Pixel Classification Layer & Class weighted cross-entropy \\
\hline & & & loss with 'Obj $1^{\prime}, \cdots,{ }^{\prime} O b j 4^{\prime}$ \\
\hline \multirow[t]{2}{*}{76} & 'skipConv3' & Convolution & $2561 \times 1$ convolutions with \\
\hline & & & stride $\left[\begin{array}{ll}2 & 2\end{array}\right]$, padding $\left[\begin{array}{llll}0 & 0 & 0 & 0\end{array}\right]$ \\
\hline 77 & 'skipBN3' & Batch Normalization & Batch normalization \\
\hline \multirow[t]{2}{*}{78} & 'skipConv2' & Convolution & $1281 \times 1$ convolutions with \\
\hline & & & stride $\left[\begin{array}{ll}2 & 2\end{array}\right]$, padding $\left[\begin{array}{llll}0 & 0 & 0 & 0\end{array}\right]$ \\
\hline 79 & 'skipBN2' & Batch Normalization & Batch normalization \\
\hline \multirow[t]{2}{*}{80} & 'skipConv1' & Convolution & $641 \times 1$ convolutions with \\
\hline & & & stride $\left[\begin{array}{ll}2 & 2\end{array}\right]$, padding $\left[\begin{array}{llll}0 & 0 & 0 & 0\end{array}\right]$ \\
\hline 81 & 'skipBN1' & Batch Normalization & Batch normalization \\
\hline
\end{tabular}


Table A.16: Proposed residual network for image decomposition with 66 layers, layers 1-12.

\begin{tabular}{|c|c|c|c|}
\hline Layer Number & Layer Name & Layer Type & Layer Description \\
\hline \multirow[t]{2}{*}{1} & 'inputImage' & Image Input & $256 \times 256 \times 3$ images with \\
\hline & & & 'zerocenter' normalization \\
\hline \multirow[t]{2}{*}{2} & 'conv1_1' & Convolution & $643 \times 3 \times 3$ convolutions with \\
\hline & & & stride $\left[\begin{array}{ll}1 & 1\end{array}\right]$, padding $\left[\begin{array}{llll}1 & 1 & 1 & 1\end{array}\right]$ \\
\hline 3 & 'bn_conv1_1' & Batch Normalization & Batch normalization \\
\hline 4 & 'relu1_1' & ReLU & ReLU \\
\hline \multirow[t]{2}{*}{5} & 'conv $1 \_2^{\prime}$ & Convolution & $643 \times 3 \times 64$ convolutions with \\
\hline & & & stride $\left[\begin{array}{ll}1 & 1\end{array}\right]$, padding $\left[\begin{array}{llll}1 & 1 & 1 & 1\end{array}\right]$ \\
\hline 6 & 'bn_conv1_2' & Batch Normalization & Batch normalization \\
\hline 7 & 'relu1_2' & ReLU & ReLU \\
\hline \multirow[t]{2}{*}{8} & 'pool1' & Max Pooling & $2 \times 2$ max pooling with stride \\
\hline & & & {$\left[\begin{array}{ll}2 & 2\end{array}\right]$, padding $\left[\begin{array}{llll}0 & 0 & 0 & 0\end{array}\right]$} \\
\hline \multirow[t]{2}{*}{9} & 'conv2_1' & Convolution & $1283 \times 3 \times 64$ convolutions with \\
\hline & & & stride $\left[\begin{array}{ll}1 & 1\end{array}\right]$, padding $\left[\begin{array}{llll}1 & 1 & 1 & 1\end{array}\right]$ \\
\hline 10 & 'bn_conv2_1' & Batch Normalization & Batch normalization \\
\hline 11 & 'relu2_1' & $\operatorname{ReLU}$ & ReLU \\
\hline \multirow[t]{2}{*}{12} & 'conv2_2' & Convolution & $1283 \times 3 \times 128$ convolutions with \\
\hline & & & stride $\left[\begin{array}{ll}1 & 1\end{array}\right]$, padding $\left[\begin{array}{llll}1 & 1 & 1 & 1\end{array}\right]$ \\
\hline
\end{tabular}


Table A.17: Proposed residual network for image decomposition, layers 13-24.

\begin{tabular}{|c|c|c|c|}
\hline Layer Number & Layer Name & Layer Type & Layer Description \\
\hline 13 & 'bn_conv2_2' & Batch Normalization & Batch normalization \\
\hline \multirow[t]{2}{*}{14} & 'add_c $2^{\prime}$ & Addition & Element-wise addition of 2 inputs \\
\hline & & & conv2_1 and $b n_{-}$conv2_2 \\
\hline 15 & 'relu2_2' & ReLU & ReLU \\
\hline \multirow[t]{2}{*}{16} & 'pool2' & Max Pooling & $2 \times 2$ max pooling with stride \\
\hline & & & {$\left[\begin{array}{ll}2 & 2\end{array}\right]$, padding $\left[\begin{array}{llll}0 & 0 & 0 & 0\end{array}\right]$} \\
\hline \multirow[t]{2}{*}{17} & 'conv $3 \_1$ ' & Convolution & $2563 \times 3 \times 128$ convolutions with \\
\hline & & & stride $\left[\begin{array}{ll}1 & 1\end{array}\right]$, padding $\left[\begin{array}{llll}1 & 1 & 1 & 1\end{array}\right]$ \\
\hline 18 & 'bn_conv3_1' & Batch Normalization & Batch normalization \\
\hline 19 & 'relu3_1' & ReLU & ReLU \\
\hline \multirow[t]{2}{*}{20} & 'conv3_2' & Convolution & $2563 \times 3 \times 256$ convolutions with \\
\hline & & & stride $\left[\begin{array}{ll}1 & 1\end{array}\right]$, padding $\left[\begin{array}{llll}1 & 1 & 1 & 1\end{array}\right]$ \\
\hline 21 & 'bn_conv3_2' & Batch Normalization & Batch normalization \\
\hline 22 & 'relu3_2' & ReLU & ReLU \\
\hline \multirow[t]{2}{*}{23} & 'conv $3 \_3{ }^{\prime}$ & Convolution & $2563 \times 3 \times 256$ convolutions with \\
\hline & & & stride $\left[\begin{array}{ll}1 & 1\end{array}\right]$, padding $\left[\begin{array}{llll}1 & 1 & 1 & 1\end{array}\right]$ \\
\hline 24 & 'bn_conv3_3' & Batch Normalization & Batch normalization \\
\hline
\end{tabular}


Table A.18: Proposed residual network for image decomposition, layers 25-36.

\begin{tabular}{|c|c|c|c|}
\hline Layer Number & Layer Name & Layer Type & Layer Description \\
\hline \multirow[t]{2}{*}{25} & 'add_c3' & Addition & Element-wise addition of 2 inputs \\
\hline & & & conv3_1 and $b n \_c o n v 3 \_3$ \\
\hline 26 & 'relu3_3' & ReLU & ReLU \\
\hline 27 & 'f $f c_{-} 1$ & Fully Connected Layer & 128 fully connected layer \\
\hline 28 & 'relufc_1' & ReLU & ReLU \\
\hline 29 & 'f $f \_-2$ & Fully Connected Layer & 128 fully connected layer \\
\hline 30 & 'relufc_2' & ReLU & ReLU \\
\hline 31 & 'f $f \_3$ & Fully Connected Layer & 256 fully connected layer \\
\hline 32 & 'relufc_3' & ReLU & ReLU \\
\hline 33 & 'decoder3_unpool' & Max Unpooling & Max Unpooling \\
\hline \multirow[t]{2}{*}{34} & 'decoder3_conv3' & Convolution & $2563 \times 3 \times 256$ convolutions with \\
\hline & & & stride $\left[\begin{array}{ll}1 & 1\end{array}\right]$, padding $\left[\begin{array}{llll}1 & 1 & 1 & 1\end{array}\right]$ \\
\hline 35 & 'decoder $3 \_b n \_3{ }^{\prime}$ & Batch Normalization & Batch normalization \\
\hline 36 & 'decoder3_relu_3' & ReLU & ReLU \\
\hline
\end{tabular}


Table A.19: Proposed residual network for image decomposition, layers 37-48.

\begin{tabular}{|c|c|c|c|}
\hline Layer Number & Layer Name & Layer Type & Layer Description \\
\hline \multirow[t]{2}{*}{37} & 'decoder3_conv2' & Convolution & $2563 \times 3 \times 256$ convolutions with \\
\hline & & & stride $\left[\begin{array}{ll}1 & 1\end{array}\right]$, padding $\left[\begin{array}{llll}1 & 1 & 1 & 1\end{array}\right]$ \\
\hline 38 & 'decoder $3 \_b n \_2$ ' & Batch Normalization & Batch normalization \\
\hline 39 & 'decoder3_relu_2' & ReLU & ReLU \\
\hline \multirow[t]{2}{*}{40} & 'decoder3_conv1' & Convolution & $1283 \times 3 \times 256$ convolutions with \\
\hline & & & stride $\left[\begin{array}{ll}1 & 1\end{array}\right]$, padding $\left[\begin{array}{llll}1 & 1 & 1 & 1\end{array}\right]$ \\
\hline 41 & 'decoder3_bn_1' & Batch Normalization & Batch normalization \\
\hline \multirow[t]{2}{*}{42} & 'add_d $3^{\prime}$ & Addition & Element-wise addition of 2 inputs \\
\hline & & & 'decoder3_conv3' and 'decoder3_bn_1' \\
\hline 43 & 'decoder3_relu_1' & ReLU & ReLU \\
\hline 44 & 'decoder2_unpool' & Max Unpooling & Max Unpooling \\
\hline \multirow[t]{2}{*}{45} & 'decoder2_conv2' & Convolution & $1283 \times 3 \times 128$ convolutions with \\
\hline & & & stride $\left[\begin{array}{ll}1 & 1\end{array}\right]$, padding $\left[\begin{array}{llll}1 & 1 & 1 & 1\end{array}\right]$ \\
\hline 46 & 'decoder $2 \_b n \_2$ ' & Batch Normalization & Batch normalization \\
\hline 47 & 'decoder2_relu_2' & ReLU & ReLU \\
\hline 48 & 'decoder2_conv1' & Convolution & $643 \times 3 \times 128$ convolutions with \\
\hline & & & stride $\left[\begin{array}{ll}1 & 1\end{array}\right]$, padding $\left[\begin{array}{llll}1 & 1 & 1 & 1\end{array}\right]$ \\
\hline
\end{tabular}


Table A.20: Proposed residual network for image decomposition, layers 49-60.

\begin{tabular}{|c|c|c|c|}
\hline Layer Number & Layer Name & Layer Type & Layer Description \\
\hline 49 & 'decoder $2 \_b n \_1$ ' & Batch Normalization & Batch normalization \\
\hline \multirow[t]{2}{*}{50} & 'add_d2' & Addition & Element-wise addition of 2 inputs \\
\hline & & & 'decoder $2 \_c o n v 2$ ' and 'decoder2_bn_1' \\
\hline 51 & 'decoder2_relu_1' & ReLU & ReLU \\
\hline 52 & 'decoder1_unpool' & Max Unpooling & Max Unpooling \\
\hline \multirow[t]{2}{*}{53} & 'decoder1_conv2' & Convolution & $643 \times 3 \times 64$ convolutions with \\
\hline & & & stride $\left[\begin{array}{ll}1 & 1\end{array}\right]$, padding $\left[\begin{array}{llll}1 & 1 & 1 & 1\end{array}\right]$ \\
\hline 54 & 'decoder $1 \_b n \_2$ ' & Batch Normalization & Batch normalization \\
\hline 55 & 'decoder1_relu_2' & ReLU & ReLU \\
\hline \multirow[t]{2}{*}{56} & 'decoder1_conv1' & Convolution & $23 \times 3 \times 64$ convolutions with \\
\hline & & & stride $\left[\begin{array}{ll}1 & 1\end{array}\right]$, padding $\left[\begin{array}{llll}1 & 1 & 1 & 1\end{array}\right]$ \\
\hline 57 & 'decoder1_bn_1' & Batch Normalization & Batch normalization \\
\hline 58 & 'decoder1_relu_1' & ReLU & $\operatorname{ReLU}$ \\
\hline 59 & 'reshape & Reshape layer & Reshape \\
\hline 60 & 'f $c_{-} 4^{\prime}$ & Fully connected layer & 2 fully connected layer \\
\hline & & & with linear activation \\
\hline
\end{tabular}


Table A.21: Proposed residual network for image decomposition, layers 61-66.

\begin{tabular}{|c|c|c|c|}
\hline Layer Number & Layer Name & Layer Type & Layer Description \\
\hline \multirow[t]{2}{*}{61} & 'skipConv3' & Convolution & $2561 \times 1$ convolutions with \\
\hline & & & stride $\left[\begin{array}{ll}1 & 1\end{array}\right]$, padding $\left[\begin{array}{llll}0 & 0 & 0 & 0\end{array}\right]$ \\
\hline 62 & 'skipBN3' & Batch Normalization & Batch normalization \\
\hline \multirow[t]{2}{*}{63} & 'skipConv2' & Convolution & $1281 \times 1$ convolutions with \\
\hline & & & stride $\left[\begin{array}{ll}1 & 1\end{array}\right]$, padding $\left[\begin{array}{llll}0 & 0 & 0 & 0\end{array}\right]$ \\
\hline 64 & 'skipBN2' & Batch Normalization & Batch normalization \\
\hline \multirow[t]{2}{*}{65} & 'skipConv1' & Convolution & $641 \times 1$ convolutions with \\
\hline & & & stride $\left[\begin{array}{ll}1 & 1\end{array}\right]$, padding $\left[\begin{array}{llll}0 & 0 & 0 & 0\end{array}\right]$ \\
\hline 66 & 'skipBN1' & Batch Normalization & Batch normalization \\
\hline
\end{tabular}




\section{Appendix B}

\section{Supervised MRF image segmentation formulation}

Define a feature set $f=\left\{f_{v}, v \in V\right\}$, where $f_{v}$ represents the set of features for each node $v$ in the image graph. The maximum a posteriori (MAP) estimate of nodes' labels is obtained as

$$
\hat{\omega}^{M A P}=\arg \max _{\omega \in \Omega} P(\omega \mid f)
$$

where $P(\omega \mid f)$ measures the probability of labeling given the observed feature $f$ and

$$
\Omega=\left\{\omega^{i}\right\}, \omega_{i}=\left\{\omega_{v}^{i} \mid \omega_{v}^{i} \in \mathcal{L}, v \in V\right\}
$$

is the set of all possible combinations of assigning $\omega_{v} \in \mathcal{L}$ to nodes $v \in V$,

$$
|\Omega|=Q^{|V|}
$$

By Bayes' theorem we have

$$
P(\omega \mid f)=\frac{P(f \mid \omega) P(\omega)}{P(f)}
$$

Using (B.4) in (B.1) and assuming i.i.d. probability distribution over $P\left(f_{v_{i}} \mid \omega_{v_{i}}\right), v_{i} \in V$, we can rewrite (B.1) as

$$
\hat{\omega}^{M A P}=\arg \max _{\omega \in \Omega} \prod_{v_{i} \in V} P\left(f_{v_{i}} \mid \omega_{v_{i}}\right) \prod_{v_{i}, v_{j} \in V, i \neq j} P\left(\omega_{v_{i}} \mid \omega_{v_{j}}\right) .
$$


Assuming we have a first-order neighborhood system (first-order conditional dependency between nodes)

$$
P\left(\omega_{v_{i}} \mid \omega_{v_{j}}, j \neq i\right)=P\left(\omega_{v_{i}} \mid \omega_{v_{j}}, v_{j} \in \mathcal{N}_{v_{i}}\right)
$$

where $\mathcal{N}_{v_{i}}$ is the neighborhood of node $v_{i}$. Therefore, (B.5) is reduced to

$$
\hat{\omega}^{M A P}=\arg \max _{\omega \in \Omega} \prod_{v_{i} \in V} P\left(f_{v_{i}} \mid \omega_{v_{i}}\right) \prod_{v_{i}, v_{j} \in V, v_{j} \in \mathcal{N}_{v_{i}}} P\left(\omega_{v_{i}} \mid \omega_{v_{j}}\right) .
$$

Therefore the final energy function to be minimized becomes [105]

$$
E(\omega)=\sum_{v_{i} \in V} \Phi_{v_{i}}\left(\omega_{v_{i}}\right)+\alpha \sum_{\left(v_{i}, v_{j}\right) \in \varepsilon_{v_{i}}} \Psi_{v_{i} v_{j}}\left(\omega_{v_{i}}, \omega_{v_{j}}\right)
$$

where $\varepsilon_{v_{i}}$ is the edge set of the image graph connected to node $v_{i} \in V, v_{j} \in V$ is a neighbor of node $v_{i}, \Phi_{v_{i}}\left(\omega_{v_{i}}\right)$ is called data-term that calculates the similarity of node $v_{i}$ to label $\omega_{v_{i}}$ and $\Psi_{v_{i} v_{j}}\left(\omega_{v_{i}}, \omega_{v_{j}}\right)$ is called regularization term that penalizes if adjacent nodes $v_{i}, v_{j}$ have different labels $\omega_{v_{i}}, \omega_{v_{j}}$. 


\section{Appendix C}

\section{Similarity Measures}

- Euclidean distance is the distance between two points in Euclidean space. Assuming to have two points $x_{s}=\left\{x_{s 1}, x_{s 2}, \ldots, x_{s N}\right\}, s=1, \ldots m$ and $y_{t}=\left\{y_{t 1}, y_{t 2}, \ldots, y_{t N}\right\}, t=1, \ldots m$ in the $N-D$ Euclidean space, the Euclidean distance is obtained by

$$
D_{s t}=\sqrt{\left(x_{s}-y_{t}\right)\left(x_{s}-y_{t}\right)^{\prime}}=\sqrt{\sum_{i=1}^{N}\left(y_{t i}-x_{s i}\right)^{2}}
$$

where $A^{\prime}$ is the transpose of $A$. A modification to Euclidean distance is standardized Euclidean distance where each term $\left(y_{t i}-x_{s i}\right)^{2}, i=1, \ldots N$ in C.1 is scaled by dividing by the standard deviation of that term

$$
D_{s t}=\sqrt{\left(x_{s}-y_{t}\right) V^{-1}\left(x_{s}-y_{t}\right)^{\prime}}
$$

where $V$ is a diagonal matrix whose $j_{t h}$ diagonal element is a scaling factor for $j_{t h}$ dimension.

- Cityblock distance is obtained by the sum of absolute values of the Cartesian coordinates of two points $x_{s}, y_{t}$

$$
D_{s t}=\Sigma_{i=1}^{N}\left|y_{t i}-x_{s i}\right|
$$

- Mahalanobis distance measures a distance between two points $x_{s}$ and $y_{t}$ of the same distribution with covariance matrix $C$

$$
D_{s t}=\sqrt{\left(x_{s}-y_{t}\right) C^{-1}\left(x_{s}-y_{t}\right)^{\prime}}
$$


- Distance correlation between two random vectors is measured by dependence of those two vectors

$$
D_{s t}=1-\frac{\left(x_{s}-\overline{x_{s}}\right)\left(y_{t}-\overline{y_{t}}\right)^{\prime}}{\sqrt{\left(x_{s}-\overline{x_{s}}\right)\left(x_{s}-\overline{\left.x_{s}\right)^{\prime}}\right.} \sqrt{\left(y_{t}-\overline{y_{t}}\right)\left(y_{t}-\overline{\left.y_{t}\right)^{\prime}}\right.}}
$$

where

$$
\overline{x_{s}}=\frac{1}{N} \Sigma_{i=1}^{N} x_{s i}
$$

and

$$
\overline{y_{t}}=\frac{1}{N} \sum_{i=1}^{N} y_{t i}
$$

- Minkowski distance which is a generalization of Euclidean distance and Cityblock distance is a distance in a normed vector space. Minkowski distance of order $p$ is obtained by

$$
D_{s t}=\sqrt[p]{\sum_{i=1}^{N}\left|x_{s i}-y_{t i}\right|^{p}}
$$

when $p=1,2$, inf Minkowski distance is the Cityblock distance, Euclidean distance, and Chebyshev distance respectively.

- Chebyshev distance is the maximum distance between two vectors along all their coordinate dimensions. Chebyshev distance is obtained by

$$
D_{s t}=\max _{i}\left\{\left|x_{s i}-y_{t i}\right|\right\}
$$

- Cosine distance or cosine similarity is measured as the cosine of the angle between two vectors

$$
D_{s t}=1-\frac{x_{s} y_{t}^{\prime}}{\sqrt{\left(x_{s} x_{s}^{\prime}\right)\left(y_{t} y_{t}^{\prime}\right)}}
$$

- Jaccard distance measures the size of the intersection divided by the size of the union of the samples as the similarity of the sample set

$$
D_{s t}=\frac{\#\left[\left(x_{s j} \neq y_{t j}\right) \cap\left(\left(x_{s j} \neq 0\right) \cup\left(y_{t j} \neq 0\right)\right)\right]}{\#\left[\left(x_{s j} \neq 0\right) \cup\left(y_{t j} \neq 0\right)\right]}
$$


- Hamming distance measures the number of dimensions in two vectors that are different

$$
D_{s t}=\frac{\#\left(x_{s j} \neq y_{t j}\right)}{N}
$$

where $N$ is the dimension of the samples.

- Spearman distance is obtained by

$$
D_{s t}=1-\frac{\left(r_{s}-\overline{r_{s}}\right)\left(r_{t}-\overline{r_{t}}\right)^{\prime}}{\sqrt{\left(r_{s}-\overline{r_{s}}\right)\left(r_{s}-\overline{r_{s}}\right)^{\prime}} \sqrt{\left(r_{t}-\overline{r_{t}}\right)\left(r_{t}-\overline{r_{t}}\right)^{\prime}}}
$$

where $r_{a j}$ is the rank of sample $A_{a j}$ over $\left.A_{1 j}, A_{2 j}, \ldots, A_{m j}\right\}$, and

$$
\begin{aligned}
& r_{a}=\left\{r_{a 1}, r_{a 2}, \ldots, r_{a N}\right\} \\
& \overline{r_{a}}=\frac{1}{N} \sum_{j=1}^{N} r_{a j}=\frac{n+1}{2}
\end{aligned}
$$




\section{Appendix D}

\section{Optimization on CNNs}

Stochastic Gradient Descent and Stochastic Gradient Descent with Momentum A simple form of gradient descent optimization can be written as

$$
W_{j}=W_{j-1}-\eta \nabla_{W} L\left(W ; x^{i} ; y^{i}\right)
$$

where $W_{j}$ is the weights for the $j^{t h}$ iteration, $\eta$ is the learning parameter and $x^{i}$ and $y^{i}$ are the $i^{t h}$ training sample and label respectively [106].

Batch gradient descent method tries to reduce the sensitivity of the optimization to the start state, as it recomputes the gradient for similar examples before updating parameters

$$
W_{j}=W_{j-1}-\eta \nabla W L\left(W ; x^{i: i+n} ; y^{i: i+n}\right), n>1
$$

In an $N$ dimensional state space $S$, gradient descent performs well when $S$ is uniformly curved towards the dimensions. However if it curves much more steeply towards one or more of the dimensions, gradient descent oscillates across the slopes and makes hesitant approach towards the local optimum.

A solution to this problem is gradient descent methods with momentum [107] where a weighted 

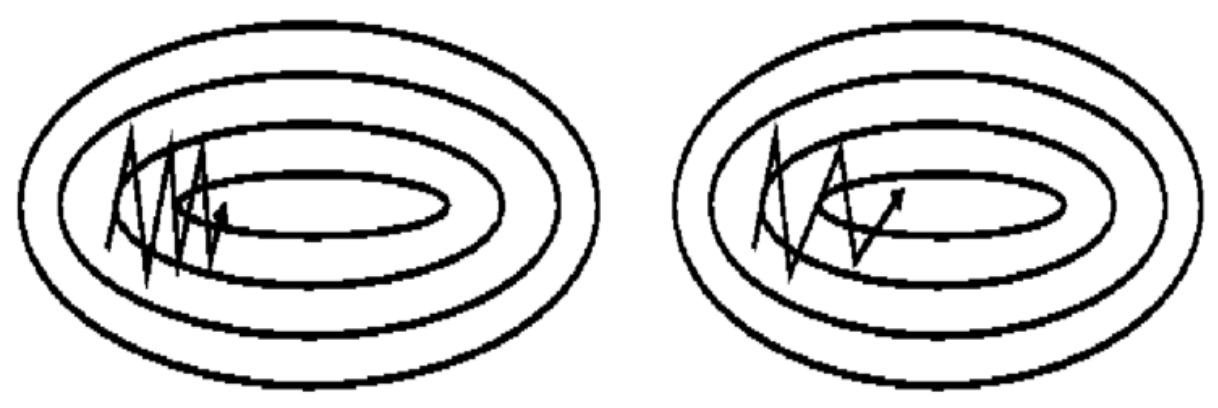

Figure D.1: Gradient descent oscillates towards local optimum when state space is curved much more steeply towards one dimension. Left: stochastic gradient descent without momentum, right: with momentum [16].

value of last update vector called momentum is added to the current update vector

$$
\begin{aligned}
& v_{t}=\beta v_{t-1}+\eta \nabla_{W} L\left(W ; x^{i: i+n} ; y^{i: i+n}\right) \\
& W=W-v_{t}
\end{aligned}
$$

where the momentum term $\beta$ is usually set to 0.9 . Figure D.1 shows Stochastic gradient descent with and without momentum where searching space is curved much more steeply towards one dimension.

However the problem with Stochastic gradient descent with momentum is that because this method follows the slope it does not knew when to slow down if the slope goes up to the hill again. A solution to this problem is Nesterov accelerated gradient method where it modifies the momentum based on the prediction of the next parameters' values instead of their current values [17]

$$
\begin{aligned}
& v_{t}=\beta v_{t-1}+\eta \nabla_{W} L\left(W-\beta v_{t-1} ; x^{i: i+n} ; y^{i: i+n}\right) \\
& W=W-v_{t}
\end{aligned}
$$

Figure D.2 shows the difference between Stochastic gradient descent with momentum with or without Nestrov parameter.

Although this method works very good, an improvement to it would be to adapt learning rate to the importance of parameters. 

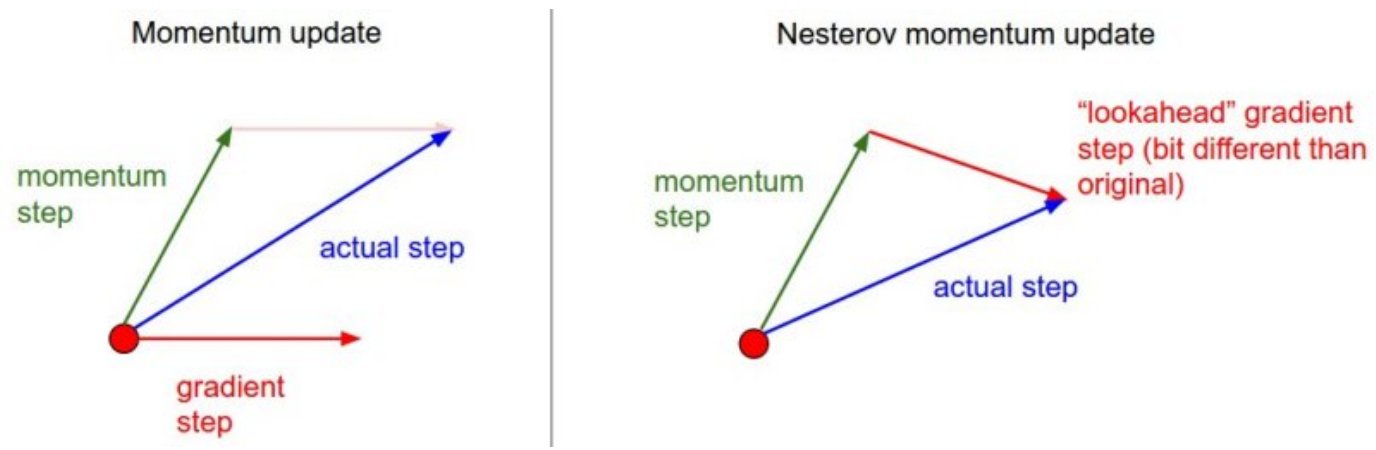

Figure D.2: Stochastic gradient descent methods with momentum. Left: original Stochastic gradient descent with momentum, right: Nestrov accelerated gradient method [17].

Adagrad Adagrad optimization method [108] adapts learning rate to the parameters by importance of the parameters. So the learning rate $g_{t, i}$ for every parameter $W_{i}$ at time $t$ is obtained by

$$
g_{t, i}=\nabla_{W_{t}} L\left(W_{t, i}\right)
$$

and the update rule becomes

$$
W_{t+1, i}=W_{t, i}-\frac{\eta}{\sqrt{G_{t, i i}+\epsilon}} g_{t, i}
$$

where $G_{t}$ is a diagonal matrix with $G_{t, i i}$ the sum of squares of gradients w.r.t $W_{i}$ up to time $t$ and $\epsilon=1 e-8$. Figure D.3 shows Adagrad optimization algorithm.

Since the squared gradients of past updates gets accumulated in the denominator, the learning rate shrinks and eventually becomes insignificantly small. A solution to this problem is Adadelta optimization algorithm.

Adadelta Adadelta optimization algorithm [109] accumulates fixed window of size $n$ of past gradients

$$
\triangle W_{t}=-\frac{\eta}{\sqrt{E\left[g^{2}\right]_{t}+\epsilon}} g_{t}
$$




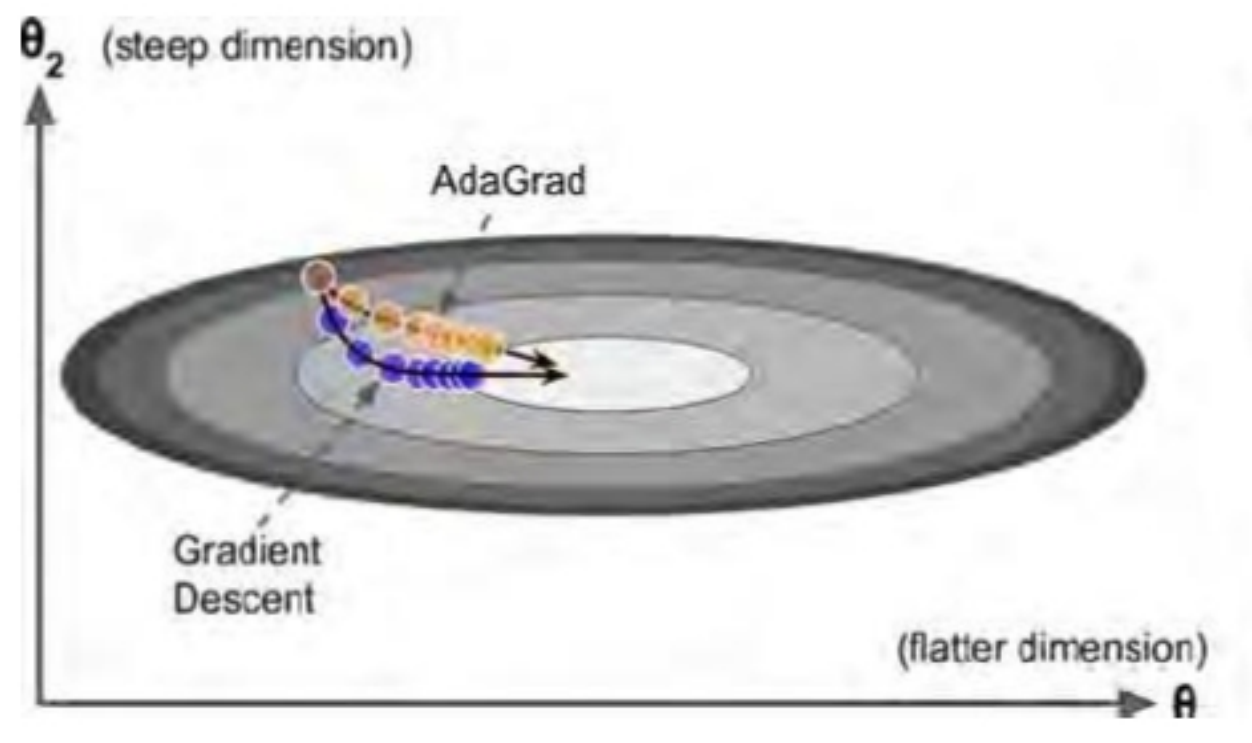

Figure D.3: Comparing Adagrad optimization method with Stochastic gradient descent method [18].

$$
E\left[g^{2}\right]_{t}=\eta E\left[g^{2}\right]_{t-1}+(1-\eta) g_{t}^{2}
$$

therefore the update rule becomes

$$
\begin{aligned}
& \triangle W_{t}=-\frac{R M S[\triangle W]_{t-1}}{R M S[g]_{t}} \\
& W_{t+1}=W_{t}+\triangle W_{t}
\end{aligned}
$$

\section{A}




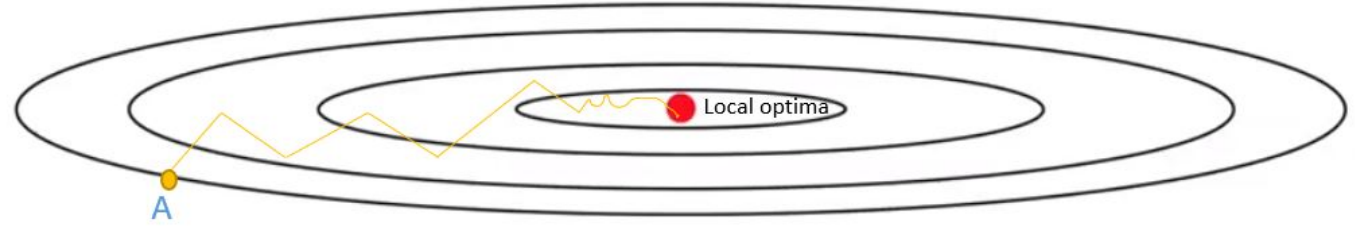

Figure D.4: RMSprop optimization method [19].

where a common value for $\eta$ is 0.001 . RMSprop method is an unpublished method proposed in Coursera classes [99]. Figure D.4 shows the RMSprop optimization algorithm.

Adam and AdaMax In addition to exponentially decaying past squared gradients average, Adam optimizer [100] has an exponentially decaying past gradients average called momentum

$$
\begin{aligned}
& W_{t+1}=w_{t}-\frac{\eta}{\sqrt{\hat{v}_{t}}+\epsilon} \hat{m}_{t} \\
& \hat{m}_{t}=\frac{m_{t}}{1-\beta_{1}^{t}} \\
& \hat{v}_{t}=\frac{v_{t}}{1-\beta_{2}^{t}} \\
& m_{t}=\beta_{1} m_{t-1}+\left(1-\beta_{1}\right) g_{t} \\
& v_{t}=\beta_{2} v_{t-1}+\left(1-\beta_{2}\right) g_{t}^{2}
\end{aligned}
$$

where $m_{t}, v_{t}$ represent the mean and variance of the gradients respectively. For fast convergence in deep learning neural networks, Adam optimizer is a good choice. Figure D.5 shows the difference between Adagrad, Adadelta, RMSprop, and Adam optimization methods.

A modification to Adam optimizer is AdaMax where the gradient is scaled inversely proportionally to $l_{p}$ norm, $p \rightarrow \infty$ instead of $l_{2}$ norm

$$
W_{t+1}=W_{t}-\frac{\eta}{\max \left(\beta_{2} v_{t-1},\left|g_{t}\right|\right.} \hat{m}_{t}
$$




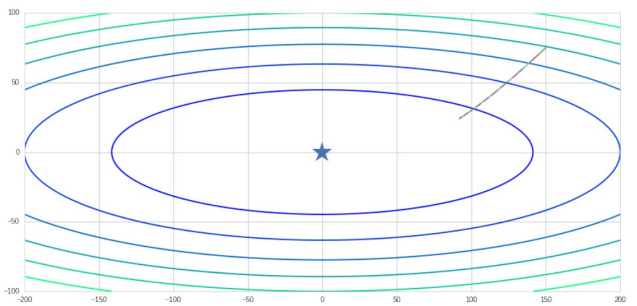

(a) Adagrad with learning rate 5

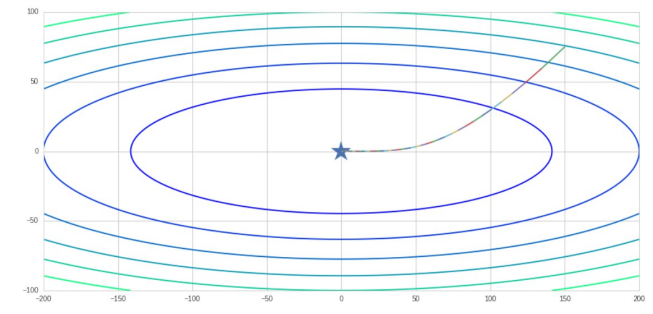

(c) RMSprop with learning rate 5

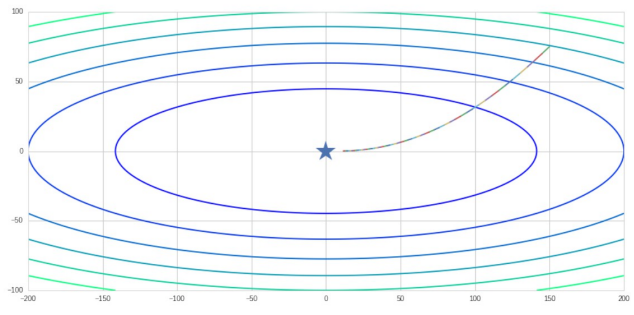

(b) Adadelta

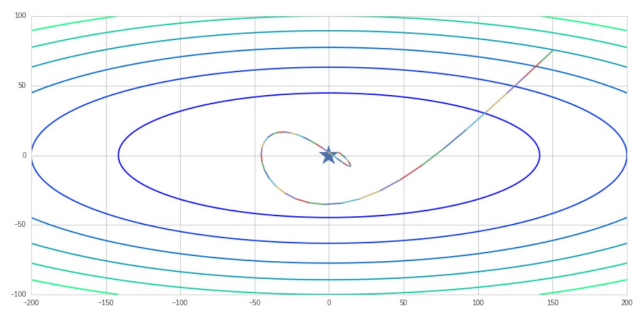

(d) Adam with learning rate 2.5

Figure D.5: Comparison between adaptive learning rate methods [20].

Nadam Adding a Nestrov momentum to the AdaMax updating rule, Nadam optimizer [110] is obtained as

$$
W_{t+1}=W_{t}-\frac{\eta}{\sqrt{\hat{v}_{t}}+\epsilon}\left(\beta_{1} \hat{m}_{t}+\frac{\left(1-\beta_{1}\right) g_{t}}{1-\beta_{1}^{t}}\right)
$$

Figure D.6 shows all the different gradient optimization methods. 


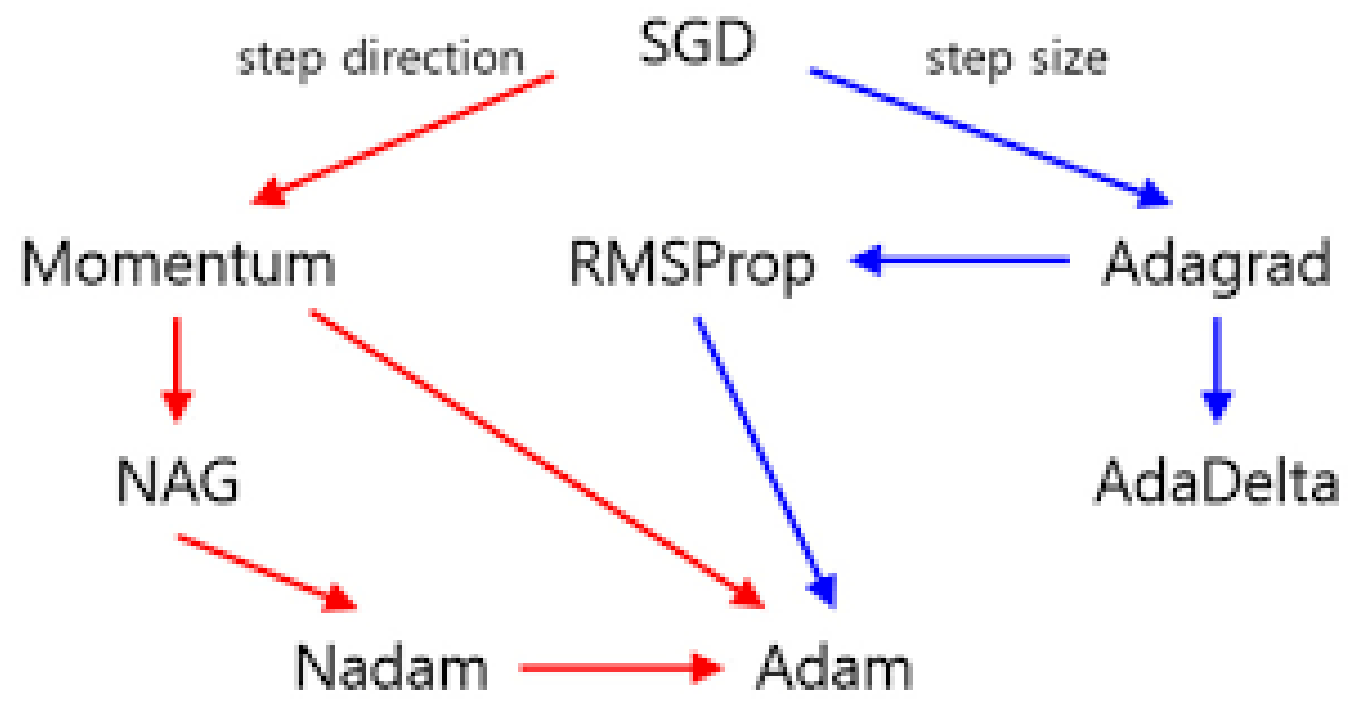

Figure D.6: Different gradient optimization methods [21]. 